\title{
ANTINEUTRINO OSCILLATIONS AND A SEARCH FOR NON-STANDARD INTERACTIONS WITH THE MINOS EXPERIMENT
}

\author{
by \\ Zeynep Isvan \\ B.S., Physics, Bosphorus University, Istanbul, Turkey, 2005 \\ M.Sc., Physics, University of Pittsburgh, 2006
}

Submitted to the Graduate Faculty of

the Kenneth P. Dietrich School of Arts and Sciences

in partial fulfillment

of the requirements for the degree of

Doctor of Philosophy

University of Pittsburgh

2012 


\section{UNIVERSITY OF PITTSBURGH DEPARTMENT OF PHYSICS AND ASTRONOMY}

This dissertation was presented

by

Zeynep Isvan

It was defended on

January 6th 2012

and approved by

Dr. Donna Naples, University of Pittsburgh, Department of Physics and Astronomy

Dr. Ayres Freitas, University of Pittsburgh, Department of Physics and Astronomy

Dr. Vittorio Paolone, University of Pittsburgh, Department of Physics and Astronomy

Dr. Lincoln Wolfenstein, Carnegie Mellon University, Department of Physics

Dr. Michael Wood-Vasey, University of Pittsburgh, Department of Physics and Astronomy

Dissertation Director: Dr. Donna Naples, University of Pittsburgh, Department of Physics

and Astronomy 
Copyright (c) by Zeynep Isvan

2012 


\title{
ANTINEUTRINO OSCILLATIONS AND A SEARCH FOR NON-STANDARD INTERACTIONS WITH THE MINOS EXPERIMENT
}

\author{
Zeynep Isvan, $\mathrm{PhD}$ \\ University of Pittsburgh, 2012
}

MINOS searches for neutrino oscillations using the disappearance of muon neutrinos from the NuMI beam at Fermilab between two detectors. The Near Detector, located near the source, measures the beam composition before flavor change occurs. The energy spectrum is measured again at the Far Detector after neutrinos travel a distance. The mixing angle and mass splitting between the second and third mass states are extracted from the energy dependent difference between the spectra at the two detectors. NuMI is able to produce an antineutrino-enhanced beam as well as a neutrino-enhanced beam. Collecting data in antineutrino-mode allows the direct measurement of antineutrino oscillation parameters. From the analysis of the antineutrino mode data we measure $\left|\Delta \bar{m}_{\mathrm{atm}}^{2}\right|=2.62_{-0.28}^{+0.31} \times 10^{-3} \mathrm{eV}^{2}$ and $\sin ^{2}(2 \bar{\theta})_{23}=0.95_{-0.11}^{+0.10}$, which is the most precise measurement of antineutrino oscillation parameters to date.

A difference between neutrino and antineutrino oscillation parameters may indicate new physics involving interactions that are not part of the Standard Model, called non-standard interactions, that alter the apparent disappearance probability. Collecting data in neutrino and antineutrino mode independently allows a direct search for non-standard interactions. In this dissertation non-standard interactions are constrained by a combined analysis of neutrino and antineutrino datasets and no evidence of such interactions is found. 


\section{TABLE OF CONTENTS}

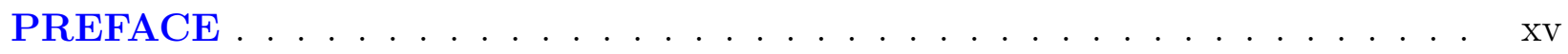

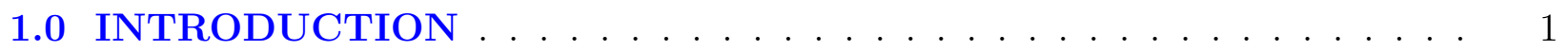

1.1 History of Neutrinos . . . . . . . . . . . . . . . . 2

1.2 The Standard Model . . . . . . . . . . . . . . . . . . . . . . 3

1.2.1 The Weak Interaction . . . . . . . . . . . . . . . . . 5

1.2.1.1 Charged-current (CC) neutrino-electron scattering $\ldots \ldots .6$

1.2.1.2 Charged-current neutrino-quark scattering . . . . . . 7

1.2.1.3 Neutral current (NC) neutrino-quark scattering . . . . . 10

1.2.2 The Electroweak Interaction . . . . . . . . . . . . . . . 12

1.2.2.1 Neutral current neutrino-electron scattering . . . . . . . . 14

1.3 Neutrino Mixing and Mass . . . . . . . . . . . . . . . . . . . 15

1.3 .1 Three Flavor Case $\ldots \ldots \ldots \ldots \ldots$

1.3 .2 Two Flavor Case . . . . . . . . . . . . . . . . . . . . . . 18

1.4 Neutrino Oscillation and $\mathcal{C P} \mathcal{T}$ Symmetry . . . . . . . . . . . . . . . 19

1.5 Neutrino Oscillations in Matter . . . . . . . . . . . . . . . 20

1.6 Non-Standard Matter Interactions . . . . . . . . . . . . . . . . 22

1.7 Status of Measurements and Remarks . . . . . . . . . . . . . . . . 25

1.7.1 Non-standard Interactions $\ldots \ldots \ldots$

2.0 THE MINOS BEAM AND DETECTORS . . . . . . . . . . . . . 31

2.1 The NuMi Beam . . . . . . . . . . . . . . . . . . . . . . . . 32

2.2 The MINOS Detectors . . . . . . . . . . . . . . . . . . . 38

$2.2 .1 \quad$ Near Detector . . . . . . . . . . . . . . . . . . 40 
$2.2 .2 \quad$ Far Detector . . . . . . . . . . . . . . . . . . . . 41

2.3 Scintillator . . . . . . . . . . . . . . . . . . . . . 42

2.4 Photomultiplier Tubes $\ldots \ldots \ldots$

2.5 The Magnetic Field . . . . . . . . . . . . . . . . . . . . . . 44

2.6 Electronics and Data Acquisition . . . . . . . . . . . . . . . 46

2.6.1 Near Detector Front-End Electronics _ . . . . . . . . . . . 46

2.6.2 Far Detector Front-End Electronics . . . . . . . . . . . . . . 51

2.6 .3 Data Acquisition . . . . . . . . . . . . . . . . . . 52

2.7 Calibration Detector . . . . . . . . . . . . . . . . . . 53

2.8 Near and Far Detector Differences . . . . . . . . . . . . . . . 56

3.0 EVENT RECONSTRUCTION, CALIBRATION AND MONTE CARLO

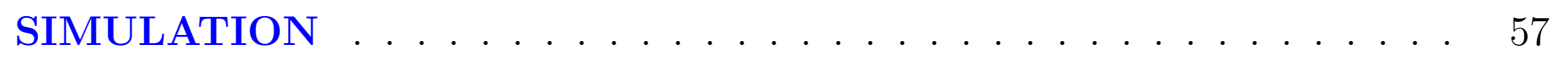

3.1 Reconstruction . . . . . . . . . . . . . . . . . . 58

3.1 .1 Slice Formation . . . . . . . . . . . . . . . 58

3.1 .2 Track Reconstruction . . . . . . . . . . . . . . . . . . . 58

3.1.3 Cluster Formation and Shower Reconstruction . . . . . . . . . 60

3.1.4 Event Formation . . . . . . . . . . . . . . . . . . . . 62

3.2 Calibration . . . . . . . . . . . . . . . . . . 62

3.2 .1 Calibration Procedure . . . . . . . . . . . . . . 63

3.2.1.1 PMT and Electronics Drift $\boldsymbol{D}_{\mathrm{PMT}}(\boldsymbol{d}, \boldsymbol{t}) \ldots \ldots$

3.2.1.2 Linearity $\boldsymbol{L}\left(\boldsymbol{d}, \boldsymbol{s}, \boldsymbol{Q}_{\text {raw }}\right) \ldots \ldots \ldots \ldots$

3.2.1.3 Scintillator Drift Correction $\boldsymbol{D}_{\text {scint }}(\boldsymbol{d}, \boldsymbol{t}) \ldots \ldots \ldots \ldots$

3.2.1.4 Strip-to-strip Calibration $\boldsymbol{S}(\boldsymbol{s}, \boldsymbol{d}, \boldsymbol{t}) \ldots \ldots \ldots$

3.2.1.5 Attenuation $\boldsymbol{A}(\boldsymbol{d}, \boldsymbol{s}, \boldsymbol{x}) \ldots \ldots \ldots \ldots$

3.2.1.6 Inter-detector Calibration $\boldsymbol{M}(\boldsymbol{d}) \ldots \ldots \ldots \ldots$

$3.2 .2 \quad$ Absolute Energy Scale . . . . . . . . . . . . . . . 66

3.3 Monte Carlo Simulation . . . . . . . . . . . . . . . . . . . 66

4.0 ANTINEUTRINO OSCILLATION ANALYSIS . . . . . . . . . 68

4.1 Event Topologies . . . . . . . . . . . . . . . . . . . . 68

4.2 Event Selection . . . . . . . . . . . . . . . . . . . . 70 
4.2 .1 Pre-selection . . . . . . . . . . . . . . . . . . . . 70

$4.2 .2 \quad$ Far Detector Fiducial Volume . . . . . . . . . . . . . 70

4.2 .3 Near Detector Fiducial Volume . . . . . . . . . . . . . . . . 71

4.2.4 Separation of charged-current and neutral-current events . . . . 72

4.3 Charge sign selection . . . . . . . . . . . . . . . . . . . . . 77

4.4 Near Detector Track End Cut . . . . . . . . . . . . . . . . 78

4.5 Beam Tuning . . . . . . . . . . . . . . . . . . . . . . . . . . . . . . 79

4.6 Near Detector Event Sample . . . . . . . . . . . . . . . . . . . 83

4.7 Near to Far Extrapolation . . . . . . . . . . . . . . . . . . . . 87

4.8 Systematic Uncertainties . . . . . . . . . . . . . . . . . . . . . . . . . . . . 91

4.8 .1 Muon energy scale . . . . . . . . . . . . . . . . . . 91

4.8 .2 Hadronic energy scale . . . . . . . . . . . . . . . . . . 92

4.8.3 Relative normalization . . . . . . . . . . . . . . . . . 93

4.8.4 Backgrounds . . . . . . . . . . . . . . . . . 94

4.8 .5 Cross section uncertainties . . . . . . . . . . . . 95

4.8.6 Smaller sources of systematic uncertainties . . . . . . . . . 95

4.9 Far Detector Selection . . . . . . . . . . . . . . . . . . 96

4.10 Fitting the Data . . . . . . . . . . . . . . . . . . 101

4.10.1 Evaluation of Oscillation Parameter Uncertainties . . . . . . . . . 105

5.0 NON-STANDARD INTERACTIONS ANALYSIS . . . . . . . . . . . 110

5.1 The Neutrino Dataset . . . . . . . . . . . . . . . . . . . . 110

5.1.1 Separation of charged-current and neutral-current events . . . . . 111

$5.1 .2 \quad$ Near Detector Sample . . . . . . . . . . . . . . . . . . 113

$5.1 .3 \quad$ Far Detector Sample . . . . . . . . . . . . . . . . 116

5.2 Antineutrino Selected Sample . . . . . . . . . . . . . . . . . . . . . 119

5.3 Near to Far Extrapolation . . . . . . . . . . . . . . . . . . . 120

5.4 Systematic uncertainties . . . . . . . . . . . . . . . . . 120

$5.5 \quad$ Fitting the Data . . . . . . . . . . . . . . . . . . . . 121

5.5.1 Evaluation of NSI parameter Uncertainties . . . . . . . . . . 128

6.0 CONCLUSION AND OUTLOOK . . . . . . . . . . . . . 132 
6.1 Future Measurements and Experiments . . . . . . . . . . . . . . . . 134

BIBLIOGRAPHY . . . . . . . . . . . . . . . . . . . . . 136 


\section{LIST OF TABLES}

1.1 Fundamental particles of the Standard Model . . . . . . . . . . . . . . . . 4

4.1 Contributions to the relative near/far normalization uncertainty $\ldots \ldots . .994$

4.2 Systematic uncertainties and their effect on the oscillation fit . . . . . . . 109

5.1 Systematic uncertainties included in the fit and their pulls . . . . . . . . 123

5.2 Systematic uncertainties and their effect on the NSI fit . . . . . . . . 129 


\section{LIST OF FIGURES}

1.1 Feynman diagrams of $\mathrm{CC} \nu-e$ and $\bar{\nu}-e$ interactions . . . . . . . 6

1.2 Feynman diagrams of $\mathrm{CC} \nu-q$ and $\bar{\nu}-q$ interactions . . . . . . . . 7

1.3 Feynman diagram of $\mathrm{NC} \nu-q$ scattering $\ldots \ldots \ldots \ldots$

1.4 Neutrino oscillation results from KamLAND . . . . . . . . . . . . . 27

2.1 Side view and bird's eye view diagrams of the locations of the MINOS detectors 31

2.2 The NuMI beamline . . . . . . . . . . . . . . . . . . . . . . . . . . . . . . . . 32

2.3 The NuMI target $\ldots \ldots \ldots \ldots$

2.4 Upstream beamline geometry and focusing optics . . . . . . . . . . . . 35

2.5 Near Detector simulated energy spectra in FHC-mode . . . . . . . . . . 36

2.6 Near Detector simulated energy spectra in RHC-mode . . . . . . . . . . . . 36

2.7 NuMI beam configurations . . . . . . . . . . . . . . . . . . . . . . . 37

2.8 MINOS detector planes . . . . . . . . . . . . . . . . . . . . . . . 38

2.9 Near Detector planes . . . . . . . . . . . . . . . . . . . . . . . . . 39

2.10 Far Detector planes . . . . . . . . . . . . . . . . . . . . . . 39

2.11 Cross sectional view of a Near Detector plane . . . . . . . . . . . . . . . 40

2.12 Cross sectional view of a MINOS scintillator strip . . . . . . . . . . . . 42

2.13 The readout chain from WLS fibers to PMTs . . . . . . . . . . . . . 43

2.14 Cross section of a Far Detector Coil _ . . . . . . . . . . . . . . . 45

2.15 Near and Far Detector magnetic field maps . . . . . . . . . . . . . . . . 45

2.16 Block diagram of the QIE chip for the Near Detector . . . . . . . . . . 47

2.17 Response of a Near Detector QIE chip . . . . . . . . . . . . . . . . . . 48

2.18 Block diagrams of a MENU and a MINDER module . . . . . . . . . . . 49 
2.19 Overview of the Near Detector front-end electronics . . . . . . . . . . . . . 50

2.20 Overview of the Far Detector front-end electronics . . . . . . . . . . . . 51

2.21 Components of the MINOS data acquisition system . . . . . . . . . . . 52

2.22 A CalDet subsection of 12 planes . . . . . . . . . . . . . . 54

2.23 CalDet response to pions and electrons . . . . . . . . . . . . . . 55

3.1 A Near Detector snarl . . . . . . . . . . . . . . . . . . 59

4.1 Event topologies of CC and NC interactions in the MINOS detectors . . . . . 69

4.2 Far Detector fiducial volume . . . . . . . . . . . . . . . . 71

4.3 Sketch demonstrating the $k \mathrm{NN}$ particle identification method . . . . . . . 73

4.4 Efficiency and purity distributions of two event selectors . . . . . . . . . . 74

4.5 Distributions of $k \mathrm{NN}$ input variables . . . . . . . . . . . . . . . 75

4.6 Distribution of $\mathrm{CC} / \mathrm{NC}$ separation parameter . . . . . . . . . . . 76

$4.7 q / p$ distribution of Near Detector events . . . . . . . . . . . . . . 77

4.8 End positions of ND events that fail the track fitter . . . . . . . . . . . 78

4.9 The reconstructed antineutrino energy distribution at the Near Detector . . . 79

4.10 Data, tuned and untuned Monte Carlo Near Detector neutrino energy distri-

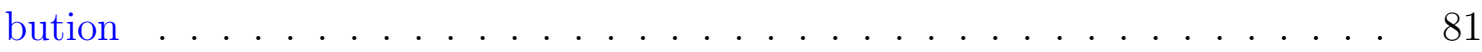

4.11 Data, tuned and untuned Monte Carlo Near Detector antineutrino energy

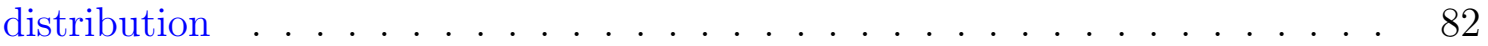

4.12 Efficiency and contamination of the Near Detector antineutrino selection . . . 83

4.13 Near Detector antineutrino energy and inelasticity distributions . . . . . . . 84

4.14 Near Detector antineutrino event position distributions . . . . . . . . . . 85

4.15 Distributions of antineutrino selection cut variables . . . . . . . . . . . 86

4.16 Acceptance of meson decay angles by the Near and Far Detectors . . . . . . . 87

4.17 FD flux over the ND flux before extrapolation . . . . . . . . . . . . . . 88

4.18 Simulated true energy spectra at the Near and Far Detectors . . . . . . . . 88

4.19 Flowchart of the beam matrix extrapolation method . . . . . . . . . . 89

4.20 Antineutrino beam matrix . . . . . . . . . . . . . . . . . 91

4.21 Systematic uncertainty on the shower energy measurement . . . . . . . . . 92

4.22 Efficiency and contamination of the Far Detector antineutrino selection . . . 97 
4.23 Far Detector antineutrino energy distributions . . . . . . . . . . . . . . 98

4.24 Far Detector antineutrino transverse position distributions . . . . . . . . . 98

4.25 Far Detector antineutrino position distributions . . . . . . . . . . . . . . 99

4.26 Far Detector antineutrino track and shower energy distributions . . . . . . 100

4.27 Far Detector distribution of antineutrino angle with respect to the beam direction 100

4.28 Feldman-Cousins surface corresponding to $90 \%$ coverage . . . . . . . . . . . . 102

4.29 Comparison of the Feldman-Cousins method to Gaussian statistics . . . . . . 103

4.30 Far Detector antineutrino data and prediction energy spectra . . . . . . . . . 104

4.31 The $68 \%, 90 \%$, and $99 \%$ antineutrino oscillation contours . . . . . . . 105

4.32 Effect of systematic uncertainties on the best oscillation fit . . . . . . . 106

4.33 The total systematic error band on the number of predicted $\bar{\nu}_{\mu}$ events $\ldots$. . 107

4.34 Comparison of systematic and statistical uncertainty . . . . . . . . . . 108

5.1 MINOS neutrino oscillation analysis allowed regions . . . . . . . . . . . 111

5.2 Distribution of neutrino $\mathrm{CC} / \mathrm{NC}$ separation variables $\ldots \ldots \ldots \ldots$

5.3 Efficiency and contamination of the Near Detector neutrino selection . . . . . 113

5.4 Near Detector neutrino energy and inelasticity distributions . . . . . . . . . . 114

5.5 Near Detector neutrino event position distributions . . . . . . . . . . . 115

5.6 Efficiency and contamination of the Far Detector neutrino selection . . . . 116

5.7 Far Detector neutrino position distributions . . . . . . . . . . . . . 117

5.8 Far Detector neutrino energy distributions _ . . . . . . . . . . . . 118

5.9 Far Detector distribution of neutrino angle with respect to the beam direction 118

5.10 Efficiency and contamination of the Near Detector antineutrino NSI selection 119

5.11 Systematic error bands from the four largest systematics . . . . . . . . . 122

5.12 Far Detector neutrino data and prediction energy spectra . . . . . . . . . . 124

5.13 Far Detector antineutrino data and prediction energy spectra . . . . . . . . 125

$5.1468 \%$ and $90 \%$ NSI contours . . . . . . . . . . . . . . . 126

5.15 One dimensional $\Delta \chi^{2}$ distributions of NSI parameters $\ldots \ldots \ldots \ldots$

5.16 Effect of systematic uncertainties on the best NSI fit . . . . . . . . . . 130

5.17 Comparison of systematic and statistical uncertainties of the NSI analysis . . 131

$6.190 \%$ C.L. contours for the published MINOS antineutrino oscillation result . 133 
6.2 The evolution of the $90 \%$ contours of antineutrino oscillation parameters with increasing data . . . . . . . . . . . . . . . . . . . . . 134 
This dissertation is dedicated to my grandfather Ahmet Vamık Isvan, whose profound appreciation for and commitment to the scientific method inspires me, and to my grandmother Cumhuriyet Reha Isvan, who taught not only me but generations of young women and men to fulfill their dreams, with infinite gratitude and love. 


\section{PREFACE}

Thanks go first and foremost to my adviser Donna Naples, an exceptional mentor, who provided all the support and guidance I needed while encouraging me to take charge and become a self-reliant researcher. She always provided insight, perspective and the big picture context.

I am indebted to my undergraduate advisor Levent Kurnaz and my professors Teoman Turgut and Engin Arik for starting me on this journey. Peter Koehler helped me discover and pursue my research field. Our graduate secretary Leyla Hirschfeld deserves much credit for making the entire process manageable. I would also like to thank the Pitt neutrino group for their collaboration.

This work would not be possible without all members of MINOS. I would particularly like to thank the NuMuBar group and its conveners Justin Evans, Jeff Hartnell, Mike Kordosky, Donna Naples and Alex Sousa. Tony Mann has pioneered the study of non-standard interactions on MINOS and offered me careful feedback at every step of my analysis. Alex Himmel deserves special thanks for his help over the years and for the documentation and code he left behind. I would like to thank Chris Backhouse, who not only had answers to all my statistics and programming questions but also the patience to explain them.

Young MINOS, my Village neighbors and the rest of the Fermilab gang, the GSA, Chivas, Two Brothers and my oldest friend Elif were always there to keep me sane; thank you all. Ulysses, thank you for making the hardest final stretch meaningful and memorable.

Finally, I would like to thank my family who have supported me throughout graduate school, often financially. You have set the bar high and always been proud of me. I would not be here without you. 


\subsection{INTRODUCTION}

The two topics addressed in this thesis are antineutrino oscillations and non-standard neutrinomatter interactions, neither of which is described by the Standard Model (SM) of particle physics. In the SM neutrinos (and antineutrinos) are massless. There is, however, evidence that they change flavor upon traveling a distance, which is only possible in vacuum if at least two of the three active neutrinos are massive. This phenomenon is called oscillation because the probability of flavor change depends on the distance traveled (and neutrino energy) and has an oscillatory shape. Neutrino oscillation experiments aim to probe the extensions to the SM which include non-zero masses. We investigate this phenomenon for antineutrinos and measure the parameters that govern their flavor change.

In neutrino oscillation experiments neutrinos travel through matter and are not propagating in vacuum. This allows other flavor change mechanisms whereby neutrinos take part in new physics interactions within and beyond the SM. While in theory such non-standard interactions (NSI) will cause flavor change even for massless neutrinos, observed flavor change effects are too large to be explained solely by NSI within their current limits. In this thesis we treat NSI as a perturbation to the oscillation model and assume the two effects jointly influence flavor change.

This chapter begins with a brief introduction to the history of neutrino physics and continues to discuss the SM, with an emphasis on weak interactions since neutrinos only interact weakly. Neutrino mass and mixing is discussed next assuming matter effects are negligible (i.e. vacuum oscillations). A discussion of standard matter effects, that is, effects from neutrino interactions described by the SM and the way these alter oscillations follows. Finally, the phenomenology of the non-standard interactions considered in this thesis, namely, flavor changing neutral current neutrino-matter interactions, is presented. The history and current 
limits of neutrino oscillation and non-standard interactions are discussed in the last section.

Chapter 2 discusses the generation and key features of the NuMI beam, the design of MINOS detectors and how these relate to the physics measurements. Chapter 3 details the process of event reconstruction and calibration as well as Monte Carlo simulation, which is key to understanding and interpreting the probabilistic processes of particle physics from the limited data statistics. Chapters 4 and 5 present detailed accounts of the antineutrino oscillation and non-standard interactions analyses, respectively, and report the findings. Chapter 6 concludes and offers a future outlook.

\subsection{HISTORY OF NEUTRINOS}

Physicists in the first two decades of the 20th century experimented with $\beta$-decay expecting the energy of the emitted electron to be unique. Repeated experiments showed, however, that the energy spectrum of electrons was continuous $[1,2,3,4,5,6]$. In the absence of another particle in the final state, such as the neutrino, the continuous spectrum of electrons appeared to violate conservation of energy. Another puzzle at the time was the observation that the Nitrogen-14 nucleus has integer spin [7, 8], an unattainable situation with the odd

number of spin- $\frac{1}{2}$ protons and electrons needed to construct an electrically neutral atom. In an attempt to solve both problems at once, Pauli proposed in his famous letter [9]:

"Electrically neutral particles, that I wish to call neutrons, which have spin $1 / 2$ and obey the exclusion principle and which further differ from light quanta in that they do not travel with the velocity of light. The mass of the neutrons should be of the same order of magnitude as the electron mass and in any event not larger than 0.01 proton masses."

Pauli was proposing a hybrid of what we know to be neutrons and neutrinos today. With the discovery of the neutron in 1932 by Chadwick [10] the neutrino gained its own identity. Fermi dubbed the particle the neutrino (Italian for the little neutral one) and developed his theory of $\beta$-decay (1968 English translation by Fred Wilson of Fermi's 1934 original [11]). The existence of the neutrino was verified experimentally by Frederick Reines and Clyde Cowan in a series of reactor experiments in the 1950s [12, 13]. Later in the decade Maurice Goldhaber et al. discovered that all neutrinos have left handed helicity [14]. The existence 
of second and third families of neutral leptons were postulated as their charged partners, the muon and tau particles, were discovered. The muon neutrino was detected in 1962 [15], which also marked the discovery of lepton number conservation; the tau neutrino was first observed by the DONuT collaboration in 2001 [16]. Properties of the $Z^{0}$ resonance indicate there are three neutrino families; if other neutrino families exist they must have masses greater than half that of the $Z$ boson [17]. Neutrinos are massless in the Standard Model.

\subsection{THE STANDARD MODEL}

The Standard Model of particle physics is a $\mathrm{SU}(3) \times \mathrm{SU}(2) \times \mathrm{U}(1)$ gauge theory which combines the color gauge group SU(3) of the strong interaction with the Glashow-WeinbergSalam (GWS) model of electroweak theory $(\mathrm{SU}(2) \times \mathrm{U}(1))$. Elementary particles that make up matter are fermions, interacting via the exchange of vector bosons. The elementary fermions comprise leptons and quarks. The electron, muon and tau particle, along with a neutrino associated with each family make up the set of leptons. Leptons are found free in nature. Quarks are not found freely; their bound states make up hadrons (three-quark states are baryons and quark-antiquark pairs are mesons). Fermions interact via three forces, the strong, electromagnetic and weak interactions. The strong interaction is responsible for nuclear binding and the interactions of the constituents of nuclei. Its mediator vector boson is called the gluon, of which there are eight. The weak interaction is responsible for radioactive decays and mediated by two charged $\left(W^{ \pm}\right)$and one neutral $\left(Z^{0}\right)$ massive vector bosons. The

electromagnetic interaction couples to all charged quarks and leptons via the photon. The 17 fundamental particles of the Standard Model are shown in Tab. 1.1.

The strong interaction is mediated by the vector boson gluon. In addition to electric charge, quarks carry the quantum number color. Gluons couple to color charge analogous to photons coupling to electric charge. Gluons themselves carry color, meaning they couple to each other unlike the photon of electromagnetism, which is electrically neutral. The field theory describing these interactions is called quantum chromodynamics (QCD). Further details of the strong interaction are left out as neutrinos do not interact strongly; extensive 


\begin{tabular}{|c|c|c|c|}
\hline Fermion & Charge & Spin & Mass $(\mathrm{MeV})$ \\
\hline$u$ & $2 / 3$ & $1 / 2$ & $1.7-3.1$ \\
$d$ & $-1 / 3$ & $1 / 2$ & $4.1-5.7$ \\
$s$ & $-1 / 3$ & $1 / 2$ & $100_{-20}^{+30}$ \\
$c$ & $2 / 3$ & $1 / 2$ & $\left(1.29_{-0.11}^{+0.05}\right) \times 10^{3}$ \\
$b$ & $-1 / 3$ & $1 / 2$ & $\left(4.19_{-0.06}^{+0.18}\right) \times 10^{3}$ \\
$t$ & $2 / 3$ & $1 / 2$ & $(172.9 \pm 0.6 \pm 0.9) \times 10^{3}$ \\
\hline$e$ & -1 & $1 / 2$ & $0.511 \pm 1.3 \times 10^{-8}$ \\
$\mu$ & -1 & $1 / 2$ & $105.7 \pm 4 \times 10^{-6}$ \\
$\tau$ & -1 & $1 / 2$ & $1776.82 \pm 0.16$ \\
$\nu_{e}$ & 0 & $1 / 2$ & $<2 \times 10^{-6}$ \\
$\nu_{\mu}$ & 0 & $1 / 2$ & $<2 \times 10^{-6}$ \\
$\nu_{\tau}$ & 0 & $1 / 2$ & $<2 \times 10^{-6}$ \\
\hline
\end{tabular}

\begin{tabular}{|c|c|c|c|}
\hline Boson & Charge & Spin & Mass $(\mathrm{GeV})$ \\
\hline$\gamma$ & 0 & 1 & 0 \\
$W^{ \pm}$ & \pm 1 & 1 & $80.399 \pm 0.023$ \\
$Z^{0}$ & 0 & 1 & $91.1876 \pm 0.0021$ \\
$g$ & 0 & 1 & 0 \\
$H^{0}$ & 0 & 0 & $>114.4$ \\
\hline
\end{tabular}

Table 1.1: Fundamental fermions and bosons of the Standard Model. Their properties are taken from [18]. 
treatments of QCD can be found in textbooks, such as [19].

The electroweak interaction is mediated by the heavy exchange bosons $W^{ \pm}$and $Z^{0}$ and the massless photon. The weak interaction is unique in that bound states formed by the weak interaction are not known. Neutrinos only take part in the weak interaction therefore the theory of weak interactions and electroweak unification are elaborated next. The discussion and notation is adapted from [20].

\subsubsection{The Weak Interaction}

Fermi's original proposal of the weak interaction to explain $\beta$-decay was modeled after the electromagnetic interaction with the matrix element,

$$
\mathcal{M}=G\left(\bar{u}_{n} \gamma^{\mu} u^{p}\right)\left(\bar{u}_{\nu_{e}} \gamma_{\mu} u_{e}\right)
$$

This vector-vector form of the interaction explained some properties of $\beta$-decay but not others. With the discovery of parity violation $\gamma^{\mu}$ is replaced with $\gamma^{\mu}\left(1-\gamma^{5}\right)$. The $\left(1-\gamma^{5}\right)$ automatically selects left-handed neutrinos (or right-handed antineutrinos). The $\beta$-decay matrix element becomes:

$$
\mathcal{M}=\frac{G}{\sqrt{2}}\left[\bar{u}_{n} \gamma^{\mu}\left(1-\gamma^{5}\right) u^{p}\right]\left[\bar{u}_{\nu_{e}} \gamma_{\mu}\left(1-\gamma^{5}\right) u_{e}\right]
$$

An interaction mediated by a spin-1 exchange boson can have a vector or an axialvector nature. If an interaction is purely vectorial or purely axial, it couples identically to right and left-handed particles. Electromagnetic interactions, for example, are purely vectorial. In parity violating interactions however, the matrix element has both a vector and an axial-vector part. The strengths of the vector and axial-vector parts are described by two coefficients, $c_{V}$ and $c_{A}$ respectively. (These coefficients are derived in Sec. 1.2.2) The closer the magnitudes of the coefficients to one another, the stronger the parity violation. Maximal parity violation occurs when $c_{V}=c_{A}$ or $c_{V}=-c_{A}$. The former case indicates that the interaction couples only to right-handed fermions (left handed antifermions) whereas in the latter case it couples only to left-handed fermions (right-handed antifermions). The weak

interaction mediated by the charged vector boson, $W^{ \pm}$is purely left handed and experimental 
evidence agrees with $c_{V}=-c_{A}$. This is called the $V-A$ theory of weak charged currents. For neutral weak currents the vectorial and axial parts are not of equal magnitude, therefore the theory is not purely $V-A$. The $V-A$ structure of the weak charged current is manifest in the following three interactions of neutrinos with electrons and quarks.
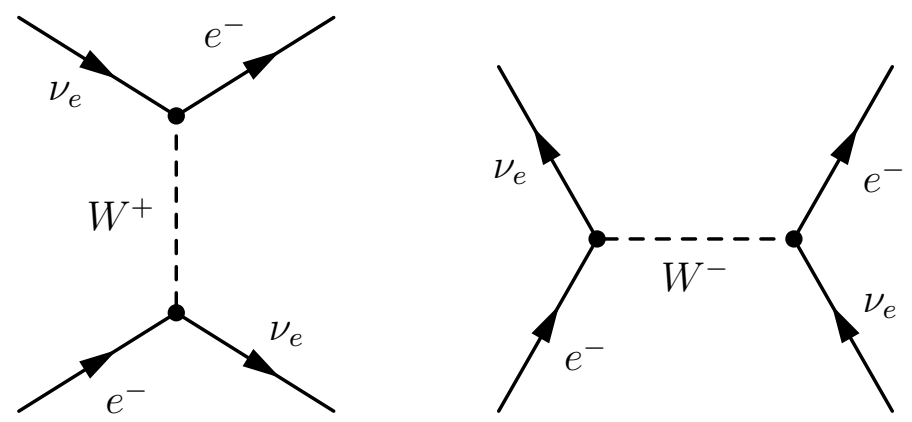

Figure 1.1: Feynman diagrams of the charged current contributions to neutrino-electron (left) and antineutrino-electron (right) scattering.

1.2.1.1 Charged-current (CC) neutrino-electron scattering Diagrams in Fig. 1.1 show CC neutrino and antineutrino scattering from electrons. The invariant amplitude for $\nu-e$ scattering is given by,

$$
\mathcal{M}=\frac{G}{\sqrt{2}}\left[\bar{u}_{\nu_{e}} \gamma^{\mu}\left(1-\gamma^{5}\right) u_{e}\right]\left[\bar{u}_{e} \gamma_{\mu}\left(1-\gamma^{5}\right) u_{\nu_{e}}\right]
$$

Squaring this amplitude and summing over the spin states of the initial electron gives, in the relativistic limit where $m_{e}=0$,

$$
\frac{1}{2} \sum_{\text {spins }}|\mathcal{M}|^{2}=16 G^{2} s^{2}
$$

where $\sqrt{s}$ is the center-of-mass energy of the neutrino-electron system. This expression is related to the differential scattering cross section via

$$
\begin{aligned}
\frac{d \sigma}{d \Omega} & =\frac{1}{64 \pi^{2} s} \frac{p_{f}}{p_{i}}|\mathcal{M}|^{2} \\
& =\frac{1}{64 \pi^{2} s}|\mathcal{M}|^{2} \\
& =\frac{G^{2} s}{4 \pi^{2}}
\end{aligned}
$$


where the $p_{f}=p_{i}$ in the relativistic limit. Integrating over the isotropic solid angle distribution yields,

$$
\sigma\left(\nu_{e} e^{-} \rightarrow \nu_{e} e^{-}\right)=\frac{G^{2} s}{\pi}
$$

The antineutrino-electron scattering diagram is merely the t-channel version of the neutrino scattering. The squared amplitude can thus be obtained by swapping $s \rightarrow t$ :

$$
\begin{aligned}
\frac{1}{2} \sum_{\text {spins }}|\mathcal{M}|^{2} & =16 G^{2} t^{2} \\
& =16 G^{2} s^{2}\left(1-\cos ^{2} \theta\right)
\end{aligned}
$$

where $\theta$ is the angle between the $\bar{\nu}$ and $e^{-}$in the laboratory frame. This gives the following expression for antineutrino-electron scattering:

$$
\frac{d \sigma\left(\bar{\nu}_{e} e^{-}\right)}{d \Omega}=\frac{G^{2} s}{16 \pi^{2}}(1-\cos \theta)^{2} \Rightarrow \sigma\left(\bar{\nu}_{e} e^{-}\right)=\frac{G^{2} s}{3 \pi}
$$

Comparing this to Eq. (1.6) it can be seen that $\sigma\left(\bar{\nu}_{e} e^{-}\right)=\frac{1}{3} \sigma\left(\nu_{e} e^{-}\right)$.
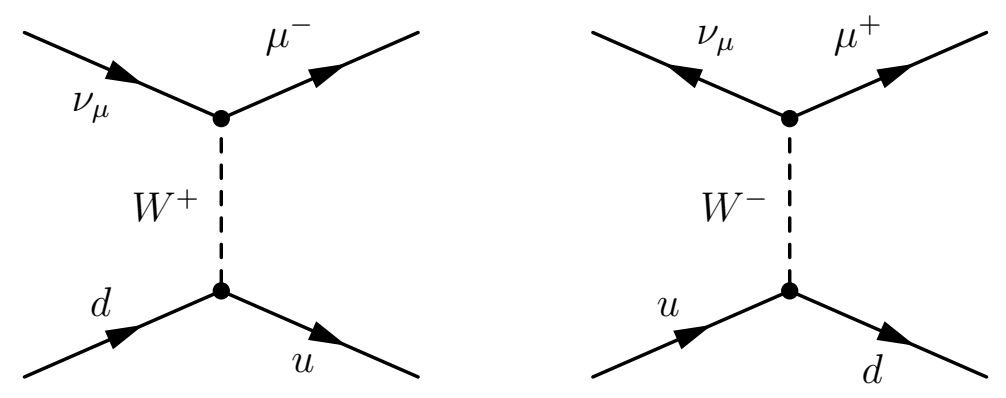

Figure 1.2: Feynman diagrams of the charged current contributions to neutrino-quark (left) and antineutrino-quark (right) scattering.

1.2.1.2 Charged-current neutrino-quark scattering Modeling the form of the quark weak currents in analogy with the neutrino-electron currents, we can write the amplitudes of the diagrams in Fig. 1.2 as: 


$$
\begin{aligned}
& \mathcal{M}_{\nu}=\frac{G}{\sqrt{2}}\left[\bar{u}_{\mu} \gamma_{\mu}\left(1-\gamma^{5}\right) u_{\nu_{\mu}}\right]\left[\bar{u}_{u} \gamma^{\mu}\left(1-\gamma^{5}\right) u_{d}\right] \\
& \mathcal{M}_{\bar{\nu}}=\frac{G}{\sqrt{2}}\left[\bar{u}_{\mu} \gamma_{\mu}\left(1-\gamma^{5}\right) u_{\bar{\nu}_{\mu}}\right]\left[\bar{u}_{d} \gamma^{\mu}\left(1-\gamma^{5}\right) u_{u}\right]
\end{aligned}
$$

Using Eq. (1.5) the differential quark scattering cross-section can be written as,

$$
\begin{aligned}
& \frac{d \sigma\left(\nu_{\mu} d \rightarrow \mu^{-} u\right)}{d \Omega}=\frac{d \sigma\left(\bar{\nu}_{\mu} \bar{d} \rightarrow \mu^{+} \bar{u}\right)}{d \Omega}=\frac{G^{2} s}{4 \pi^{2}} \\
& \frac{d \sigma\left(\bar{\nu}_{\mu} u \rightarrow \mu^{+} d\right)}{d \Omega}=\frac{d \sigma\left(\nu_{\mu} \bar{u} \rightarrow \mu^{-} \bar{d}\right)}{d \Omega}=\frac{G^{2} s}{16 \pi^{2}}(1+\cos \theta)^{2}
\end{aligned}
$$

Backward scattering (i.e. $\theta=\pi$ ) for processes of the form (1.11) is not allowed. Because of the pure $V-A$ structure of weak charged currents only left handed $u$ and $d$ (and right handed $\bar{u}$ and $\bar{d}$ ) quarks couple. Charge conservation requires that $\nu_{\mu}$ do not couple to $u$ or $\bar{d}$ quarks while $\bar{\nu}_{\mu}$ do not interact with $\bar{u}$ and $d$ type quarks.

To embed the constituent $\nu-q$ scattering cross section in the experimentally observable neutrino-nucleon $(\nu-N)$ inclusive cross-section the following Lorentz-invariant kinematic quantities are defined:

- Energy transfer to the hadronic system

$$
\nu=\frac{p \cdot q}{M}
$$

- Negative squared four-momentum of the exchange boson

$$
Q^{2}=-q^{2}=-\left(k_{1}-k_{2}\right)^{2}
$$

- Inelasticity

$$
y=\frac{p \cdot q}{p \cdot k_{1}}
$$

- Bjorken scaling variable

$$
x=\frac{Q^{2}}{2 p \cdot q}
$$

- Squared center of mass energy of the neutrino-nucleon system

$$
s=\left(p+k_{1}\right)^{2}
$$


where $k_{1}, k_{2}, q, p$ are the 4-momenta of the neutrino, muon, $\mathrm{W}$ and the nucleon respectively. $M$ is the mass of the nucleon. In the laboratory frame ${ }^{1}$,

$$
\begin{aligned}
x & =\frac{Q^{2}}{2 M \nu} \\
1-y & =\frac{1}{2}(1+\cos \theta)
\end{aligned}
$$

where $\theta$ is the scattering angle in the lab frame. The constituent quarks carry a fraction $x s$ of the center of mass momentum of the system, with which the cross-sections of Eq. (1.11) can be written:

$$
\begin{aligned}
& \frac{d \sigma\left(\nu_{\mu} d \rightarrow \mu^{-} u\right)}{d y}=\frac{G^{2} x s}{4 \pi} \\
& \frac{d \sigma\left(\bar{\nu}_{\mu} u \rightarrow \mu^{+} d\right)}{d y}=\frac{G^{2} x s}{4 \pi}(1-y)^{2}
\end{aligned}
$$

The neutrino-nucleon scattering inclusive cross section is given by

$$
\frac{d \sigma(\nu N \rightarrow \mu X)}{d x d y}=\sum_{i} f_{i}(x)\left(\frac{d \sigma_{i}}{d y}\right)
$$

where $X$ is the hadronic shower and $f_{i}(x)$ are the parton distribution functions of the up and down quarks in the nucleon. Neutrinos only interact with $d$ and $\bar{u}$ quarks. Assuming there are equal numbers of protons and neutrons in matter, the parton distribution functions can be given as:

$$
\begin{aligned}
& d^{\text {proton }}(x)+d^{\text {neutron }}(x)=d^{\text {proton }}(x)+u^{\text {proton }}(x) \equiv Q(x) \\
& \bar{u}^{\text {proton }}(x)+\bar{u}^{\text {neutron }}(x)=\bar{u}^{\text {proton }}(x)+\bar{d}^{\text {proton }}(x) \equiv \bar{Q}(x)
\end{aligned}
$$

where $Q(x)$ and $\bar{Q}(x)$ denote the quark and antiquark distributions in matter respectively. With these distribution functions the expression in (1.20) can be expanded to:

\footnotetext{
${ }^{1} x$ is an invariant quantity for which the following equality holds in all frames
} 


$$
\begin{aligned}
\frac{d \sigma\left(\nu N \rightarrow \mu^{-} X\right)}{d x d y} & =Q(x) \frac{d \sigma\left(\nu_{\mu} d \rightarrow \mu^{-} u\right)}{d y}+\bar{Q}(x) \frac{d \sigma\left(\nu_{\mu} \bar{u} \rightarrow \mu^{-} \bar{d}\right)}{d y} \\
& =\frac{G^{2} x s}{\pi}\left[Q(x)+(1-y)^{2} \bar{Q}(x)\right]
\end{aligned}
$$

Similarly, the $\bar{\nu}_{\mu}$-nucleon interaction cross section is given by:

$$
\begin{aligned}
\frac{d \sigma\left(\bar{\nu} N \rightarrow \mu^{+} X\right)}{d x d y} & =\bar{Q}(x) \frac{d \sigma\left(\bar{\nu}_{\mu} \bar{d} \rightarrow \mu^{+} \bar{u}\right)}{d y}+Q(x) \frac{d \sigma\left(\bar{\nu}_{\mu} u \rightarrow \mu^{+} d\right)}{d y} \\
& =\frac{G^{2} x s}{\pi}\left[\bar{Q}(x)+(1-y)^{2} Q(x)\right]
\end{aligned}
$$

Assuming there are no antiquarks in matter, that is, $\bar{Q}=0$, the ratio of the integrated $\nu$ nucleon and $\bar{\nu}$-nucleon charged current scattering cross sections is $\sigma(\bar{\nu}) / \sigma(\nu)=\frac{1}{3}$. In reality there is about a $5 \%$ antiquark component in a proton. The cross-section expressions (1.23) and (1.24) allow for the computation of $Q$ and $\bar{Q}$ from experimental data.

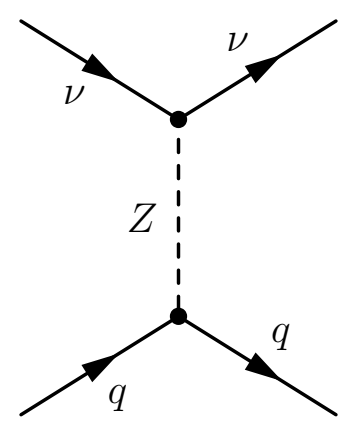

Figure 1.3: Feynman diagram of the neutral current contribution to neutrino-quark scattering.

1.2.1.3 Neutral current (NC) neutrino-quark scattering The Feynman diagram representing neutral current neutrino-quark scattering is shown in Fig. 1.3. Neutral currents 
are not pure $V-A$ for quarks, unlike charged weak currents (i.e. $c_{V}^{q} \neq c_{A}^{q}$ in the quark vertex in the following equation). The amplitude of of $\mathrm{NC} \nu-q$ scattering is given by:

$$
\mathcal{M}=\frac{G_{N}}{\sqrt{2}}\left[\bar{u}_{\nu} \gamma^{\mu}\left(1-\gamma^{5}\right) u_{\nu}\right]\left[\bar{u}_{q} \gamma_{\mu}\left(c_{V}^{q}-\gamma^{5} c_{A}^{q}\right) u_{q}\right]
$$

where $G_{N}$ is the weak neutral current coupling constant (experimentally $G_{N} \simeq G$ ). Because weak neutral currents are not pure $V-A, c_{V} \neq c_{A}$ in general. This allows weak neutral currents to have a right handed component, though this is known to be small from experiment. The constituent cross-sections contributing to the total neutral current cross-section are:

$$
\begin{aligned}
& \frac{d \sigma\left(\nu_{L} d_{L}\right)}{d y}=\frac{G_{N}^{2} x s}{\pi} \frac{\left(c_{V}^{d}+c_{A}^{d}\right)^{2}}{4} \\
& \frac{d \sigma\left(\nu_{L} \bar{u}_{R}\right)}{d y}=\frac{G_{N}^{2} x s}{\pi}(1-y)^{2} \frac{\left(c_{V}^{u}-c_{A}^{u}\right)^{2}}{4} \\
& \frac{d \sigma\left(\nu_{L} d_{R}\right)}{d y}=\frac{G_{N}^{2} x s}{\pi} \frac{\left(c_{V}^{d}-c_{A}^{d}\right)^{2}}{4} \\
& \frac{d \sigma\left(\nu_{L} \bar{u}_{L}\right)}{d y}=\frac{G_{N}^{2} x s}{\pi}(1-y)^{2} \frac{\left(c_{V}^{u}+c_{A}^{u}\right)^{2}}{4}
\end{aligned}
$$

With the definitions

$$
g_{L}=\frac{1}{2}\left(c_{V}+c_{A}\right), \quad g_{R}=\frac{1}{2}\left(c_{V}-c_{A}\right)
$$

the total inclusive neutral current $\nu-q$ scattering cross section can be obtained as,

$$
\frac{d \sigma(\nu N \rightarrow \nu X)}{d x d y}=\frac{G_{N}^{2} x s}{\pi}\left[g_{L}^{2}\left(Q(x)+(1-y)^{2} \bar{Q}(x)\right)+g_{R}^{2}\left(\bar{Q}(x)+(1-y)^{2} Q(x)\right)\right]
$$

Integrating equations (1.23), (1.24) and (1.31) over $x$ gives the following $\mathrm{NC}$ and $\mathrm{CC}$ neutrino-nucleon interaction cross sections: 


$$
\begin{aligned}
& \frac{d \sigma^{N C}(\nu)}{d y}=\frac{G_{N}^{2} s}{\pi}\left\{g_{L}\left[Q+(1-y)^{2} \bar{Q}\right]+g_{R}\left[\bar{Q}+(1-y)^{2} Q\right]\right\} \\
& \frac{d \sigma^{N C}(\bar{\nu})}{d y}=\frac{G_{N}^{2} s}{\pi}\left\{g_{L}\left[\bar{Q}+(1-y)^{2} Q\right]+g_{R}\left[Q+(1-y)^{2} \bar{Q}\right]\right\} \\
& \frac{d \sigma^{C C}(\nu)}{d y}=\frac{G^{2} s}{\pi}\left[Q+(1-y)^{2} \bar{Q}\right] \\
& \frac{d \sigma^{C C}(\bar{\nu})}{d y}=\frac{G^{2} s}{\pi}\left[\bar{Q}+(1-y)^{2} Q\right]
\end{aligned}
$$

While weak neutral currents are not purely $V-A, g_{R}$ is calculated and experimentally measured to be small. On the other hand, CC interactions are believed to be purely $V-A$.

\subsubsection{The Electroweak Interaction}

In order to convert weak interactions to a renormalizable theory, weak currents need to form a symmetry group. The weak charge-raising and charge-lowering currents can be written,

$$
\begin{aligned}
& J_{\mu}^{+}=\bar{\chi}_{L} \gamma_{\mu} \tau_{+} \chi_{L} \\
& J_{\mu}^{-}=\bar{\chi}_{L} \gamma_{\mu} \tau_{-} \chi_{L}
\end{aligned}
$$

where

$$
\chi_{L}=\left(\begin{array}{c}
\nu_{L} \\
l^{-}
\end{array}\right)_{L}
$$

is the lepton doublet and $\tau_{ \pm}=\frac{1}{2}\left(\tau_{1} \pm \tau_{2}\right)$ are the tau matrices, with Pauli spin matrices $\tau_{i}$. The charged currents form an SU(2) isospin triplet with the following neutral current

$$
\begin{aligned}
J_{\mu}^{3} & =\bar{\chi}_{L} \gamma_{\mu} \frac{1}{2} \tau_{3} \chi_{L} \\
& =\frac{1}{2} \bar{\nu}_{l L} \gamma_{\mu} \nu_{l L}-\bar{l}_{L} \gamma_{\mu} l_{L} .
\end{aligned}
$$


This current can be seen to have only a left-handed component unlike the neutral current of $\nu-q$ scattering, which has a non-zero right-handed component. The electromagnetic current has both left- and right-handed components, and can be incorporated into the above neutral current by the definition of a weak hypercharge current such that,

$$
j_{\mu}^{e m}=J_{\mu}^{3}+j_{\mu}^{Y} \text { (hypercharge). }
$$

This expands the symmetry group to $\mathrm{SU}(2)_{L} \times \mathrm{U}(1)_{Y}$. Two symmetry groups require two coupling constants, therefore a constant is needed in addition to $e$. To develop electroweak theory analogous to QED, it is assumed that electroweak currents are coupled to vector bosons just as the electromagnetic current is coupled to the photons: a triplet of vector fields $W_{\mu}^{i}$ coupled to $J_{\mu}^{i}$ with strength $g$ and a singlet vector field $B_{\mu}$ coupled to the weak hypercharge current $j_{\mu}^{Y}$ with strength $g^{\prime} / 2$ :

$$
-i g J^{i \mu} W_{\mu}^{i}-i \frac{g^{\prime}}{2} j^{Y \mu} B_{\mu}
$$

where $W_{ \pm}=\sqrt{\frac{1}{2}}\left(W_{\mu}^{1} \mp W_{\mu}^{2}\right)$ are the massive charged bosons and $W_{\mu}^{3}$ and $B_{\mu}$ are the neutral fields.

The electromagnetic current is a combination of $J_{\mu}^{3}$ and $j_{\mu}^{Y}$ and the physical states $A_{\mu}$ and $Z_{\mu}$ are mixtures of the neutral gauge fields $W_{\mu}^{3}$ and $B_{\mu}$ with mixing angle $\theta_{W}$ :

$$
\begin{aligned}
& A_{\mu}=B_{\mu} \cos \theta_{W}+W_{\mu}^{3} \sin \theta_{W} \\
& Z_{\mu}=-B_{\mu} \sin \theta_{W}+W_{\mu}^{3} \cos \theta_{W}
\end{aligned}
$$

With Eq. (1.40) the electroweak neutral current becomes,

$$
\begin{aligned}
& -i\left(g \sin \theta_{W} J_{\mu}^{3}+g^{\prime} \cos \theta_{W} \frac{j_{\mu}^{Y}}{2}\right) A_{\mu} \\
& -i\left(g \cos \theta_{W} J_{\mu}^{3}-g^{\prime} \cos \theta_{W} \frac{j_{\mu}^{Y}}{2}\right) Z_{\mu}
\end{aligned}
$$


The first term is the electromagnetic interaction, therefore

$$
g \sin \theta_{W}=g^{\prime} \cos \theta_{W}=e .
$$

Plugging this back into the expression (1.41) the weak neutral current interaction is given by,

$$
-i \frac{g}{\cos \theta_{W}}\left(J_{\mu}^{3}-\sin ^{2} \theta_{W} j_{\mu}^{e m}\right) Z_{\mu}
$$

which can be expressed as

$$
-i \frac{g}{\cos \theta_{W}} \bar{\psi}\left[\gamma^{\mu} \frac{1}{2}\left(c_{V}-c_{A} \gamma^{5}\right)\right] \psi Z_{\mu}
$$

with

$$
\begin{aligned}
& c_{V}=T^{3}-2 \sin ^{2} \theta_{W} Q \\
& c_{A}=T^{3}
\end{aligned}
$$

where $T^{3}$ is the third component of weak isospin and $Q$ is the electric charge.

\subsubsection{Neutral current neutrino-electron scattering Processes such as $\nu_{\mu} e^{-} \rightarrow$} $\nu_{\mu} e^{-}$can only occur via neutral currents since the $W^{ \pm}$bosons will not couple a second generation lepton, $\nu_{\mu}$, to an electron. In analogy with neutral current neutrino-quark scattering discussed above, the differential cross section can be expressed as,

$$
\frac{d \sigma\left(\nu_{\mu} e^{-}\right)}{d y}=\frac{G_{N}^{2} s}{4 \pi}\left[\left(c_{V}+c_{A}\right)^{2}+(1-y)^{2}\left(c_{V}-c_{A}\right)^{2}\right] .
$$

Integrated, this gives:

$$
\begin{aligned}
& \sigma(\nu e)=\frac{G_{N}^{2} s}{3 \pi}\left(c_{V}^{2}+c_{V} c_{A}+c_{A}^{2}\right) \\
& \sigma(\bar{\nu} e)=\frac{G_{N}^{2} s}{3 \pi}\left(c_{V}^{2}-c_{V} c_{A}+c_{A}^{2}\right)
\end{aligned}
$$

In the case of $c_{V}=c_{A}, \sigma(\bar{\nu}) / \sigma(\nu)=\frac{1}{3}$ in agreement with experiment. 


\subsection{NEUTRINO MIXING AND MASS}

The concept of neutrino flavor change allowed by massive neutrinos was first considered by Bruno Pontecorvo [21]. His oscillation model suggested a transition of the type $\nu \rightleftharpoons \bar{\nu}$ : at the time only one lepton family was known. When the muon neutrino was discovered he considered transitions from electron to muon flavor as well. The formalism of neutrino mixing was developed in 1962 by Ziro Maki, Masami Nakagawa and Shoichi Sakata as a two flavor model [22]. The following discussion first generalizes this to an arbitrary number of neutrino families then considers the special cases of three and two flavors.

Neutrino oscillations occur because neutrino flavor eigenstates $\left|\nu_{\alpha}\right\rangle$ are not identical to mass eigenstates $\left|\nu_{i}\right\rangle$ but mixtures of them. Neutrinos interact via the weak interaction in flavor eigenstates and propagate in vacuum in mass eigenstates. In the following discussion Greek subscripts denote flavor eigenstates and Latin subscripts denote mass eigenstates.

A neutrino which is created at time $t=0$ in a weak flavor $\left|\nu_{\alpha}(0)\right\rangle$ eigenstate can be expressed as a sum of mass eigenstates:

$$
\left|\nu_{\alpha}(0)\right\rangle=\sum_{i} U_{\alpha i}^{*}\left|\nu_{i}\right\rangle
$$

where $U$ is the mixing matrix.

Mass eigenstates are eigenstates of the Hamiltonian and evolve as such while the neutrino propagates:

$$
\left|\nu_{\alpha}(t)\right\rangle=\sum_{i} U_{\alpha i}^{*} e^{i p_{i} \cdot x}\left|\nu_{i}\right\rangle
$$

where $x$ is the position of the neutrino and $p_{i}$ is the four-momentum of the mass state $i$.

The neutrino is detected at time $t$ in a detector via its weak interaction, in a flavor eigenstate $\left\langle\nu_{\beta}\right|=\sum_{j} U_{\beta j}\left\langle\nu_{j}\right|$. The overlap with the state at time $t=0$ is given by:

$$
\begin{aligned}
\left\langle\nu_{\beta} \mid \nu_{\alpha}(t)\right\rangle & =\sum_{j} \sum_{i} U_{\beta j} U_{\alpha i}^{*} e^{i p_{i} \cdot x}\left\langle\nu_{j} \mid \nu_{i}\right\rangle \\
& =\sum_{i} U_{\beta i} U_{\alpha i}^{*} e^{i p_{i} \cdot x} .
\end{aligned}
$$


With $E_{i}$ and $m_{i}$ denoting the energy and mass of the $i^{\text {th }}$ mass eigenstate and assuming that all mass eigenstates have the same three-momemtum $\mathbf{p}$,

$$
\begin{aligned}
p_{i} \cdot x & =E_{i} t-\mathbf{p} \cdot \mathbf{x} \\
& =t \sqrt{|\mathbf{p}|^{2}+m_{i}^{2}}-\mathbf{p} \cdot \mathbf{x} .
\end{aligned}
$$

For ultra-relativistic neutrinos with $m_{i}<<E_{i}$ we can make the assumptions $t=L$ and $\mathbf{p} \cdot \mathbf{x}=|\mathbf{p}| L$, where $L$ is the distance traveled. With these the above expression becomes:

$$
\begin{aligned}
p_{i} \cdot x & =|\mathbf{p}| L\left(1+\frac{m_{i}^{2}}{2|\mathbf{p}|^{2}}\right)-|\mathbf{p}| L \\
& =\frac{m_{i} L}{2 E}
\end{aligned}
$$

This gives the amplitude $\left\langle\nu_{\beta} \mid \nu_{\alpha}(L)\right\rangle=\sum_{i} U_{\beta i} U_{\alpha i}^{*} e^{i \frac{m_{i}^{2} L}{2 E}}$. The probability of observing a neutrino in flavor $\beta$ after traveling a distance $L$ can then be calculated as,

$$
\begin{aligned}
P\left(\nu_{\alpha} \rightarrow \nu_{\beta}\right) & =\left|\left\langle\nu_{\beta} \mid \nu_{\alpha}(L)\right\rangle\right|^{2} \\
& =\left|\sum_{i} U_{\beta i} U_{\alpha i}^{*} e^{i \frac{m_{i}^{2} L}{2 E}}\right|^{2} \\
& =\left(\sum_{j} U_{\beta j}^{*} U_{\alpha j} e^{-i \frac{m_{j}^{2} L}{2 E}}\right)\left(\sum_{i} U_{\beta i}^{*} U_{\alpha i} e^{-i \frac{m_{i}^{2} L}{2 E}}\right) \\
& =\sum_{i} \sum_{j} U_{\beta j}^{*} U_{\beta i} U_{\alpha i}^{*} U_{\alpha j} e^{-i \frac{\Delta m_{i j}^{2} L}{2 E}}
\end{aligned}
$$

where $\Delta m_{i j}^{2}=m_{i}^{2}-m_{j}^{2}$. 
Using the unitarity of $U$ and performing some algebra we can obtain

$$
\begin{aligned}
P\left(\nu_{\alpha} \rightarrow \nu_{\beta}\right) & =\delta_{\alpha \beta}+2 \sum_{i>j} \sum_{j}\left[U_{\beta j}^{*} U_{\beta i} U_{\alpha i}^{*} U_{\alpha j}\left(e^{-i \frac{\Delta m_{i j}^{2} L}{2 E}}-1\right)\right] \\
& =\delta_{\alpha \beta} \\
& +2 \sum_{i>j} \sum_{j} \mathfrak{I m}\left[U_{\beta j}^{*} U_{\beta i} U_{\alpha i}^{*} U_{\alpha j}\right] \sin \left(\frac{\Delta m_{i j}^{2} L}{2 E}\right) \\
& +4 \sum_{i>j} \sum_{j} \mathfrak{R e}\left[U_{\beta j}^{*} U_{\beta i} U_{\alpha i}^{*} U_{\alpha j}\right] \sin ^{2}\left(\frac{\Delta m_{i j}^{2} L}{4 E}\right) .
\end{aligned}
$$

Eq. (1.55) gives the probability that a neutrino generated in flavor eigenstate $\alpha$ will be found in flavor eigenstate $\beta$ a distance $L$ away from the source, for any number of flavor eigenstates. Note that this transition probability is only nonzero when $\Delta m_{i j}^{2}$ is nonzero.

\subsubsection{Three Flavor Case}

There are three known flavors of leptons and three active flavors of neutrinos. Three flavor mixing can be expressed as:

with

$$
\left(\begin{array}{c}
\nu_{e} \\
\nu_{\mu} \\
\nu \tau
\end{array}\right)=U\left(\begin{array}{l}
\nu_{1} \\
\nu_{2} \\
\nu_{3}
\end{array}\right)
$$

$$
\begin{aligned}
U & =\left(\begin{array}{lll}
U_{e 1} & U_{e 2} & U_{e 3} \\
U_{\mu 1} & U_{\mu 2} & U_{\mu 3} \\
U_{\tau 1} & U_{\tau 2} & U_{\tau 3}
\end{array}\right) \\
& =\left(\begin{array}{ccc}
1 & 0 & 0 \\
0 & c_{23} & s_{23} \\
0 & -s_{23} & c_{23}
\end{array}\right)\left(\begin{array}{ccc}
c_{13} & 0 & s_{13} e^{-i \delta} \\
0 & 1 & 0 \\
-s_{13} e^{i \delta} & 0 & c_{13}
\end{array}\right)\left(\begin{array}{ccc}
c_{12} & s_{12} & 0 \\
-s_{12} & c_{12} & 0 \\
0 & 0 & 1
\end{array}\right) \\
& =\left(\begin{array}{ccc}
c_{12} c_{13} \\
s_{13} e^{-i \delta} \\
s_{12} c_{23}-c_{12} s_{23} s_{13} e^{i \delta} & c_{12} c_{23}-s_{12} s_{23} s_{13} e^{i \delta} & s_{23} c_{13} \\
s_{12} s_{23}-c_{12} c_{23} s_{13} e^{i \delta} & -c_{12} s_{23}-s_{12} c_{23} s_{13} e^{i \delta} & c_{23} c_{13}
\end{array}\right)
\end{aligned}
$$

where $s_{i j}=\sin \theta_{i j}$ and $c_{i j}=\cos \theta_{i j}$ and $\delta$ is the CP-violating phase. Majorana phases are omitted since they do not contribute to neutrino oscillations. 


\subsubsection{Two Flavor Case}

Because of the large difference between the two mass splittings, experiments such as MINOS are sensitive to the mixing between two of the three flavors which justifies the following two flavor approximation:

$$
\left(\begin{array}{c}
\nu_{\mu} \\
\nu_{\tau}
\end{array}\right)=\left(\begin{array}{cc}
\cos \theta & \sin \theta \\
-\sin \theta & \cos \theta
\end{array}\right)\left(\begin{array}{c}
\nu_{2} \\
\nu_{3}
\end{array}\right)
$$

By the same arguments and approximations that led to Eq. (1.55) in the general $n$-flavor case, with two flavors a state $|\nu(0)\rangle=\left|\nu_{\mu}\right\rangle=\cos \theta\left|\nu_{2}\right\rangle+\sin \theta\left|\nu_{3}\right\rangle$ will evolve in time $t$, or equivalently path length traveled, $L$, to

$$
|\nu(L)\rangle=\left|\nu_{\mu}\right\rangle=\cos \theta \exp \left(-i \frac{m_{2}^{2} L}{2 E}\right)\left|\nu_{2}\right\rangle+\sin \theta \exp \left(-i \frac{m_{3}^{2} L}{2 E}\right)\left|\nu_{3}\right\rangle
$$

The probability that it will be detected with the same flavor after traveling this distance is given by

$$
\begin{aligned}
P_{\nu_{\mu} \rightarrow \nu_{\mu}} & =\left|\left\langle\nu_{\mu} \mid \nu(L)\right\rangle\right|^{2}=\left|\cos ^{2} \theta \mathrm{e}^{-\frac{i m_{2}^{2} L}{2 E}}+\sin ^{2} \theta \mathrm{e}^{-\frac{i m_{3}^{2} L}{2 E}}\right|^{2} \\
& =1-2 \cos ^{2} \theta \sin ^{2} \theta\left[1-\cos \left(\frac{\Delta m_{23}^{2} L}{2 E}\right)\right] \\
& =1-\sin ^{2} 2 \theta\left[2 \sin ^{2}\left(\frac{\Delta m_{23}^{2} L}{4 E}\right)\right]
\end{aligned}
$$

In SI units with $c$ and $\hbar$ restored the argument of the sine becomes

$$
\frac{\Delta m_{23}^{2} c^{4} L}{4 E \hbar c}=1.27 \frac{\Delta m_{23}^{2} L}{E}\left[\frac{\mathrm{eV}^{2} \cdot \mathrm{km}}{\mathrm{GeV}}\right]
$$

Substituting this into Eq. (1.60) gives the survival probability relevant to MINOS

$$
P_{\nu_{\mu} \rightarrow \nu_{\mu}}=1-\sin ^{2}\left(2 \theta_{23}\right) \sin ^{2}\left(1.27 \frac{\Delta m_{23}^{2} L}{E}\right)
$$




\subsection{NEUTRINO OSCILLATION AND $\mathcal{C P} \mathcal{T}$ SYMMETRY}

The discovery of the preferred helicity of the neutrino by Goldhaber [14] demonstrates a violation of both parity, $\mathcal{P}$ and charge conjugation, $\mathcal{C}$. The charge conjugation operator changes the left handed neutrino to a left handed antineutrino which is not observed in nature. This suggests nature could be symmetric under the application of $\mathcal{C P}$, which transforms a left handed neutrino to a right handed antineutrino. However, in the neutral kaon system, the observation of the $\mathcal{C P}$-odd $K_{L}^{0}$ state decaying into three pions through the $\mathcal{C P}$-even channel posits direct evidence of $\mathcal{C P}$ violation [23].

It is a fundamental result of quantum field theory that nature must be invariant under the combined operation of $\mathcal{C P} \mathcal{T}$, where $\mathcal{T}$ denotes time reversal, with the assumption that nature also obeys unitarity and locality, is Lorentz invariant, and spacetime is flat [24]. $\mathcal{C P} \mathcal{T}$ invariance means that the masses and lifetimes of particles and antiparticles are equal. The current limit from the mass difference of $K^{0}$ and $\bar{K}^{0}$ is [18]

$$
\frac{\left|m_{K^{0}}-m_{\bar{K}^{0}}\right|}{m_{\text {average }}}<10^{-18} \quad(90 \% \text { c.l. }) \text {. }
$$

Assuming conservation of $\mathcal{C P} \mathcal{T}, \mathcal{C P}$ violation could manifest itself as a violation of $\mathcal{T}$ symmetry. Applying the operator $\mathcal{T}$ to neutrino oscillations is equivalent to taking the complex conjugate of the PMNS matrix, which amounts to changing the sign of the $\mathcal{C P}$ violating phase $\delta$. This phase only appears with the $\theta_{13}$ term whose measurement requires a $\nu_{e}$ appearance signal. In disappearance experiments, the measured quantity is the survival probability, which involves only the modulus squared of the matrix elements and sensitivity to $\delta$ is obscured. A violation of $\mathcal{C P} \mathcal{T}$ in the neutrino sector would show up as a difference between neutrino and antineutrino oscillation parameters, $\theta_{i j} \neq \bar{\theta}_{i j}$ or $\Delta m_{i j}^{2} \neq \Delta \bar{m}_{i j}^{2}$. There are numerous models where neutrino and antineutrino oscillations are investigated in the context of $\mathcal{C P} \mathcal{T}$ (see, for example, [25, 26, 27]). In this work $\mathcal{C P} \mathcal{T}$ invariance is assumed; antineutrinos are compared to neutrinos with the consideration that their interactions with matter along the travel path may be different. 


\subsection{NEUTRINO OSCILLATIONS IN MATTER}

Neutrino oscillations are altered by the presence of matter along the path of travel from creation to detection as first shown by Lincoln Wolfenstein in 1978 [28]. The model was later

applied to the solar neutrino oscillation problem by Mikheev and Smirnov [29] and is known as the MSW effect after its three discoverers. This effect is different for $\nu_{e}$ because matter is predominantly composed of leptons in the first family (electrons). While all three flavors of neutrinos experience the same neutral current scattering in matter, electron neutrinos also scatter via charged current coherent forward scattering. In the absence of matter the evolution of the neutrino state is expressed in the mass basis by,

$$
i \frac{\partial}{\partial t}\left(\begin{array}{c}
\nu_{1} \\
\nu_{2}
\end{array}\right)=H_{0}\left(\begin{array}{c}
\nu_{1} \\
\nu_{2}
\end{array}\right)
$$

where, for mass eigenstates the Hamiltonian is obtained

$$
H_{0}=\left(\begin{array}{cc}
E_{1} & 0 \\
0 & E_{2}
\end{array}\right) \approx\left(\begin{array}{cc}
p+\frac{m_{1}^{2}}{2 E} & 0 \\
0 & p+\frac{m_{2}^{2}}{2 E}
\end{array}\right)
$$

where $p$ is the neutrino momentum, $E$ is the neutrino energy, and $m_{1}$ and $m_{2}$ are the masses of the eigenstates $\nu_{1}$ and $\nu_{2}$. Mass eigenstates can be expressed as flavor eigenstates multiplied by $U^{\dagger}$ from the left. Then multiplying the expression from the left by $U$ gives,

$$
\begin{aligned}
i \frac{\partial}{\partial t}\left(\begin{array}{c}
\nu_{e} \\
\nu_{x}
\end{array}\right) & =U H_{0} U^{\dagger}\left(\begin{array}{c}
\nu_{e} \\
\nu_{x}
\end{array}\right) \\
& =\left(\begin{array}{cc}
p+\frac{m_{1}^{2}}{2 E} & 0 \\
0 & p+\frac{m_{2}^{2}}{2 E}
\end{array}\right)\left(\begin{array}{c}
\nu_{e} \\
\nu_{x}
\end{array}\right)
\end{aligned}
$$

Charged-current scattering of electron neutrinos from electrons in matter introduces an additional matter potential,

$$
V_{e}= \pm \sqrt{2} G_{F} N e
$$


where $N_{e}$ is the electron density in matter and the matter potential changes sign from positive to negative for antineutrinos. The charged-current matter interaction only affects electron neutrinos and is flavor conserving, therefore its only contribution is to the $e-e$ component. This yields the total Hamiltonian,

$$
\begin{aligned}
H & =U H_{0} U^{\dagger}+\left(\begin{array}{cc}
V_{e} & 0 \\
0 & 0
\end{array}\right) \\
& =\frac{\Delta m^{2}}{2 E}\left(\begin{array}{cc}
\sin ^{2} \theta+\frac{2 E}{\Delta m^{2}} V_{e} & -\sin \theta \cos \theta \\
-\sin \theta \cos \theta & \cos ^{2} \theta
\end{array}\right)+\ldots
\end{aligned}
$$

where $\Delta m^{2}=m_{2}^{2}-m_{1}^{2}$ and terms proportional to the identity matrix denoted by "..." are ignored. Diagonalizing the Hamiltonian, it becomes

$$
H=\left(\begin{array}{cc}
\cos \theta_{m} & -\sin \theta_{m} \\
\sin \theta_{m} & \cos \theta_{m}
\end{array}\right)\left(\begin{array}{cc}
-\Delta m_{m}^{2} & 0 \\
0 & \Delta m_{m}^{2}
\end{array}\right)\left(\begin{array}{cc}
\cos \theta_{m} & \sin \theta_{m} \\
-\sin \theta_{m} & \cos \theta_{m}
\end{array}\right)
$$

where we have introduced $\theta_{m}$ and $\Delta m_{m}^{2}$ as the effective mixing angle and mass splitting in the presence of matter interactions. These effective parameters can be expressed in terms of the vacuum oscillation parameters as,

$$
\begin{aligned}
\sin ^{2} 2 \theta_{m} & =\frac{\sin ^{2} 2 \theta}{\left(\cos ^{2} 2 \theta \pm \frac{2 \sqrt{2} E G_{F} N_{e}}{\Delta m^{2}}\right)^{2}+\sin ^{2} 2 \theta} \\
\Delta m_{m}^{2} & =\Delta m^{2}\left(\left(\cos ^{2} 2 \theta \pm \frac{2 \sqrt{2} E G_{F} N_{e}}{\Delta m^{2}}\right)^{2}+\sin ^{2} 2 \theta\right)^{\frac{1}{2}}
\end{aligned}
$$

This means that in matter neutrinos oscillate with effective oscillation parameters $\Delta m_{m}^{2}$ and $\sin ^{2} 2 \theta_{m}$, which are obtained by modifying the vacuum oscillation parameters depending on neutrino energy and matter density. A resonance occurs at $\left(\cos ^{2} 2 \theta-\frac{2 \sqrt{2} E G_{F} N_{e}}{\Delta m_{21}^{2}}\right)=0$ that results in maximal mixing, i.e. $\theta_{m}=\pi / 4$ even if vacuum mixing is small [30]. This gives rise to a much greater rate of conversion from $\nu_{e}$ to $\nu_{\mu}$ than otherwise possible. 


\subsection{NON-STANDARD MATTER INTERACTIONS}

As discussed in section 1.5, standard matter interactions affect the survival probability of the electron neutrino but not of the muon and tau neutrinos since muon and tau particle densities in normal matter are minuscule compared to electrons. There can be, however, non-standard matter interactions (NSI) that influence survival probabilities of second and third generation neutrinos. These are interactions of neutrinos with matter (up and down quarks and electrons), standard versions of which have been discussed in Section 1.2.1. One way in which non-standard interactions can arise is through the violation of the unitarity of the lepton mixing matrix in certain see-saw models [31]. Sizable NSI effects can be introduced via new scalar exchange bosons such as Higgs bosons or scalar leptoquarks, some supersymmetric models may also give rise to NSI [32].

In general there can be charged-current NSI which affect the production and detection of neutrinos, and neutral-current NSI which alter the propagation through matter. The general leptonic charged current NSI Lagrangian density is given by [33],

$$
\mathcal{L}_{\mathrm{NSI}}^{l}=-2 \sqrt{2} G_{F} \varepsilon_{\alpha \beta}^{\gamma \delta}\left[\bar{l}_{\alpha} \gamma^{\mu}\left(1+a \gamma^{5}\right) l_{\beta}\right]\left[\bar{\nu}_{\gamma} \gamma_{\mu}\left(1-\gamma^{5}\right) \nu_{\delta}\right]
$$

where $a=+/-1$ for right/left handed leptons and $\alpha \neq \beta$ to generate flavor changing interactions. This leptonic CC NSI is relevant to the production of neutrinos. Similarly, non-standard charged-current interactions with quarks can be expressed by,

$$
\mathcal{L}_{\mathrm{NSI}}^{u}=-2 \sqrt{2} G_{F} \varepsilon_{\alpha \beta}^{u d} V_{u d}\left[\bar{u} \gamma^{\mu}\left(1+a \gamma^{5}\right) d\right]\left[\bar{l}_{\alpha} \gamma_{\mu}\left(1-\gamma^{5}\right) \nu_{\beta}\right]+\text { h.c. }
$$

where matter contains only $u$ and $d$ quarks. Neutrinos interact with $d$ or $\bar{u}$ quarks, antineutrinos with $u$ or $\bar{d}$ (see Section 1.2.1.2). The interaction with quarks is relevant to the production and detection of neutrinos. In this thesis charged-current contributions to non-standard interactions are neglected because they are strongly constrained from shortbaseline neutrino oscillation experiments [34, 35] and MINOS is not sensitive to them [36]. While in general they can be as strong as neutral-current NSI, NC NSI are enhanced by the length of the baseline and are the only NSI interactions able to generate a detectable effect in MINOS. 
The neutral-current NSI Lagrangian is of the form [37],

$$
\mathcal{L}_{\mathrm{NSI}}=-\frac{G_{F}}{\sqrt{2}} \sum_{\substack{f=u, d, e \\ a= \pm 1}} \varepsilon_{\alpha \beta}^{f a}\left[\bar{u}_{\nu_{\alpha}} \gamma^{\mu}\left(1-\gamma^{5}\right) u_{\nu_{\beta}}\right]\left[\bar{u}_{f} \gamma_{\mu}\left(1+a \gamma^{5}\right) u_{f}\right]
$$

where coefficients $\varepsilon_{\alpha \beta}^{f}$ give the strength of the NSI effect on transitions between $\alpha$ and $\beta$ flavors as a result of interacting with fermion $f=e, u, d$. Since matter is not polarized only the vectorial part of this interaction is non-vanishing for propagation through matter; the NSI Lagrangian will add a term to the Hamiltonian as,

$$
H_{\mathrm{NSI}}=V_{e}\left(\begin{array}{ccc}
\varepsilon_{e e} & \varepsilon_{e \mu} & \varepsilon_{e \tau} \\
\varepsilon_{e \mu}^{*} & \varepsilon_{\mu \mu} & \varepsilon_{\mu \tau} \\
\varepsilon_{e \tau}^{*} & \varepsilon_{\mu \tau}^{*} & \varepsilon_{\tau \tau}
\end{array}\right)
$$

where $V_{e}= \pm \sqrt{2} G_{F} N_{e}$ is the matter potential. The $\varepsilon$ parameters have been summed over the fermion contributions

$$
\varepsilon_{\alpha \beta}=\sum_{f, a} \varepsilon_{\alpha \beta}^{f a} \frac{N_{f}}{N_{e}}
$$

where $N_{f}$ is the fermion density in matter.

MINOS is sensitive to oscillations in the atmospheric sector and the vacuum oscillation model has been developed in the two-flavor approximation in section 1.3. The NSI formalism will be discussed in such a two flavor scenario as well. The parameters that appear in the following discussion are described as

- Real valued NSI strength parameters $\varepsilon=\varepsilon_{\mu \tau}$ and $\varepsilon^{\prime}=\varepsilon_{\mu \mu}-\varepsilon_{\tau \tau}$

- Atmospheric mass splitting $\Delta m^{2}=\Delta m_{32}^{2}=m_{3}^{2}-m_{2}^{2}$ and mixing angle $\theta=\theta_{23}$

- Neutrino energy $E=E_{\nu}$

The two flavor Hamiltonian including NSI can be expressed as,

$$
H=\left(\begin{array}{cc}
\cos (2 \theta) \frac{\Delta m^{2}}{4 E}+\varepsilon^{\prime} V_{e} & \sin (2 \theta) \frac{\Delta m^{2}}{4 E}+\varepsilon V_{e} \\
\sin (2 \theta) \frac{\Delta m^{2}}{4 E}+\varepsilon V_{e} & -\cos (2 \theta) \frac{\Delta m^{2}}{4 E}+\varepsilon^{\prime} V_{e}
\end{array}\right)
$$


Using the general formalism for two-flavor oscillations is presented in [38], any Hamiltonian can be expressed as,

$$
H=\mathbf{H} \cdot \boldsymbol{\sigma}
$$

where $\boldsymbol{\sigma}=\left(\sigma_{1}, \sigma_{2}, \sigma_{3}\right)$ are the Pauli matrices. The oscillation probability from flavor $\alpha$ to $\beta$ is then be given by

$$
P_{\alpha \beta}=\sin ^{2}(2 \tilde{\theta}) \sin ^{2}(k t)
$$

where

$$
\begin{aligned}
\sin ^{2}(2 \tilde{\theta}) & =\frac{H_{1}^{2}+H_{2}^{2}}{|\mathbf{H}|^{2}} \\
k t & \approx k L=|\mathbf{H}|=\sqrt{\sum_{i=1}^{3} H_{i}^{2}} .
\end{aligned}
$$

Applying this to the NSI Hamiltonian with

$$
H_{1}=\frac{\Delta m^{2}}{4 E} \sin (2 \theta)+\varepsilon V, \quad H_{2}=0, \quad H_{3}=\frac{\Delta m^{2}}{4 E} \cos (2 \theta)+\varepsilon^{\prime} V
$$

the following expressions are obtained:

$$
\begin{aligned}
& \sin ^{2}(2 \tilde{\theta})=\frac{\left(\frac{\Delta m^{2}}{4 E}\right)^{2} \sin ^{2}(2 \theta)+\left(\varepsilon V_{e}\right)^{2}+2\left(\frac{\Delta m^{2}}{4 E}\right) \sin (2 \theta) \varepsilon V_{e}}{\left(\frac{\Delta m^{2}}{4 E}\right)^{2}+2\left(\frac{\Delta m^{2}}{4 E}\right)\left[\sin (2 \theta) \varepsilon V_{e}+\cos (2 \theta) \varepsilon^{\prime} V_{e}\right]+\left(\varepsilon^{\prime} V_{e}\right)^{2}+\left(\varepsilon V_{e}\right)^{2}} \\
& \sin ^{2}(k t)=\left[\left(\frac{\Delta m^{2}}{4 E}\right)^{2}+2\left(\frac{\Delta m^{2}}{4 E}\right)\left[\sin (2 \theta) \varepsilon V+\cos (2 \theta) \varepsilon^{\prime} V\right]\left(\varepsilon^{\prime} V_{e}\right)^{2}+\left(\varepsilon V_{e}\right)^{2}\right]^{1 / 2} L
\end{aligned}
$$

In the analysis presented in this dissertation the effect of flavor-changing NSI are investigated while the flavor conserving $\varepsilon^{\prime}$ is not considered. The sensitivity to off-diagonal flavor-changing $\varepsilon$ is greater due to the possible interference between standard and nonstandard amplitudes $[39,28,40,41,42,43,44]$. With this reduction and some trigonometric rearrangement the survival probability becomes 


$$
\begin{aligned}
P\left(\nu_{\mu} \rightarrow \nu_{\mu}\right)=1- & {\left[1-\frac{\cos ^{2}(2 \theta)}{1 \pm \sin ^{2}(2 \theta)\left(\frac{4 E}{\Delta m^{2}}\right) \varepsilon V+\left(\frac{4 E}{\Delta m^{2}} \varepsilon V\right)^{2}}\right] } \\
& \sin ^{2}\left(\left[\left(\frac{4 E}{\Delta m^{2}}\right)^{2} \pm 2 \sin (2 \theta)\left(\frac{4 E}{\Delta m^{2}}\right) \varepsilon V+(\varepsilon V)^{2}\right]^{1 / 2} L\right)
\end{aligned}
$$

where $V=\left|V_{e}\right|$ and the sign is denoted explicitly, positive for neutrinos and negative for antineutrinos.

\subsection{STATUS OF MEASUREMENTS AND REMARKS}

Standard neutrino oscillations are governed by six parameters, $\Delta m_{21}^{2}, \theta_{12}, \Delta m_{32}^{2}, \theta_{23}, \theta_{13}$ and $\delta_{\mathrm{CP}}$. The first four are known with precision. There is an upper limit on $\theta_{13}$ showing it to be small but there is no conclusive evidence for whether or not it differs from zero. Since the determination of the CP-violating phase, $\delta_{\mathrm{CP}}$, depends on the value of $\theta_{13}$, this parameter is entirely unconstrained at present.

Neutrino disappearance was first observed by Ray Davis in 1968 at his Homestake mine experiment [45]. Davis's experiment was designed to measure the solar neutrino flux from the high energy neutrinos produced in the sun in ${ }^{8} \mathrm{~B}$ decay by searching for an excess of Argon atoms produced in a tank of $\mathrm{Cl}_{2} \mathrm{Cl}_{4}$ through the reaction $\nu_{e}+{ }^{37} \mathrm{Cl} \rightarrow e^{-}+{ }^{37} \mathrm{Ar}$. He measured the flux to be less than half that predicted by the standard solar model (SSM). The most likely cause of the observed discrepancy was thought to be a problem with the solar model. The Kamiokande experiment verified the discrepancy with an independent experiment [46] and showed that the reduced flux is observed for atmospheric neutrinos as well as solar neutrinos. The Kamiokande detector was sensitive to the same high energy neutrinos as Homestake and reported that the observed flux is $0.46 \pm 0.15$ times the SSM prediction.

The $p p$ solar chain, in which Hydrogen nuclei become ${ }^{4} \mathrm{He}$ by fusion is better understood, but produces neutrinos at a lower energy. These neutrinos can be observed in Gallium via $\nu_{e}+{ }^{71} \mathrm{Ga} \rightarrow e^{-}+{ }^{72}$ Ge with a threshold of $0.233 \mathrm{MeV}$. Two experiments, SAGE and 
GALLEX, performed this measurement in the 1990s [47, 48] and confirmed that the observed flux is about half that of the SSM prediction. Kamiokande and later its upgrade with larger fiducial volume, Super-Kamiokande, kept confirming that the measured $\nu_{e}$ flux is half of the SSM prediction $[49,50]$ : this came to be known as the "solar neutrino problem."

The SNO [51] experiment could differentiate between $\mathrm{CC}$ and NC interactions. Only $\nu_{e}$ participate in the former while all flavors participate equally in the latter. This confirmed that the total flux is in agreement with the SSM prediction while the number of electron neutrinos is lower than expected, demonstrating agreement with both the SSM and neutrino flavor change.

Another way of measuring neutrino oscillations in the "solar" sector is the detection of $\nu_{e}$ from other sources. Nuclear reactors provide a flux of $\bar{\nu}_{e}$ and KamLAND is one of the early experiments to detect reactor antineutrinos [52]. The KamLAND detector in Japan is sensitive to the same region as the solar neutrino experiments above. Fig. 1.4 shows contours from solar experiments along with KamLAND results. The combined fit to all existing data in the solar sector yields the best world measurement of

$$
\Delta m_{21}^{2}=(7.59 \pm 0.20) \times 10^{-5} \mathrm{eV}^{2}, \tan ^{2} \theta_{12}=0.47_{0.05}^{0.06}
$$

The mixing angle is near maximal, and this region is dubbed the large mixing angle (LMA) region. From the sign of MSW matter effects, solar neutrino oscillation measurements indicate that $m_{2}>m_{1}$.

The second set of oscillation parameters, $\Delta m_{32}^{2}$ and $\theta_{23}$, are measured from atmospheric neutrinos and accelerator based $\nu_{\mu}$ beams. Pions and kaons are produced when cosmic rays strike the atmosphere and they decay into neutrinos via, primarily, $\pi^{+} \rightarrow \mu^{+}+\nu_{\mu} ; \mu^{+} \rightarrow$ $e^{+}+\nu_{e}+\bar{\nu}_{\mu}$. Therefore, it is natural to expect a $\nu_{\mu}: \nu_{e}$ flux ratio of 2:1. However, a similar discrepancy to the solar neutrino anomaly was observed when experiments confirmed the expected $\nu_{e}$ rate but reported that the $\nu_{\mu}$ flux was $\frac{2}{3}$ the prediction [53]. Atmospheric neutrino oscillation is now a well established phenomenon with precision measurements of the parameters governing it. The most recent and precise measurement using neutrinos from 


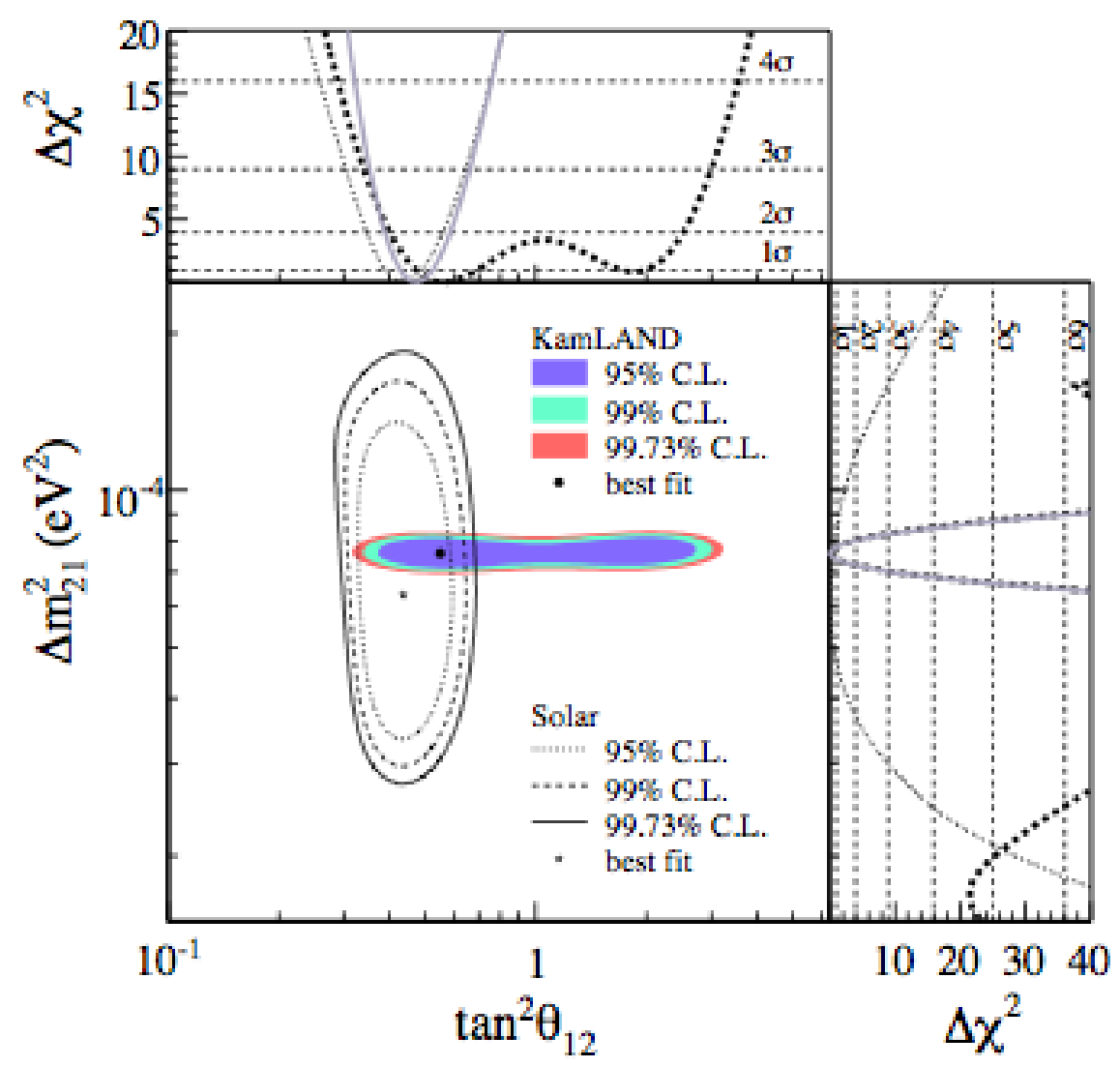

Figure 1.4: Allowed regions for neutrino oscillations from combined solar experiments and KamLAND. The side panels show the $\Delta \chi^{2}$ profiles. From [52].

the atmosphere come from Super-Kamiokande (assuming $m_{3}>m_{1,2}$ ) [54]

$$
\begin{gathered}
1.9 \times 10^{-3} \leq\left|\Delta m_{\text {atm }}^{2}\right| \leq 2.6 \times 10^{-3} \mathrm{eV}^{2} \quad(90 \% \text { C.L }) \\
0.407 \leq \sin ^{2} \theta_{23} \leq 0.583 \quad(90 \% \text { C.L })
\end{gathered}
$$

with best fit values $\left|\Delta m_{\mathrm{atm}}^{2}\right|=2.1 \times 10^{-3} \mathrm{eV}^{2}$ and $\sin ^{2} \theta_{23}=0.5$.

These results are confirmed by experiments using manmade atmospheric neutrinos, that 
is, accelerator based muon neutrino beams. MINOS measures

$$
\left|\Delta m^{2}\right|=2.32_{-0.08}^{+0.12} \times 10^{-3} \mathrm{eV}^{2} \quad \sin ^{2}\left(2 \theta_{23}\right)>0.90 \quad(90 \% \text { C.L })
$$

making the most precise $(5 \%)$ measurement of the mass splitting.

The last mixing angle, $\theta_{13}$ is yet to be determined definitively, but there are constraints on its value. A non-zero $\theta_{13}$ implies electron neutrino disappearance similar to muon neutrino disappearance with survival probability

$$
P_{\nu_{e} \rightarrow \nu_{e}}=1-\sin ^{2}\left(2 \theta_{13}\right) \sin ^{2}\left(1.27 \Delta m_{\mathrm{atm}}^{2} \frac{L}{E}\right)
$$

where $L$ is in meters, $E$ in $\mathrm{MeV}$ and $\Delta m_{31}^{2} \approx \Delta m_{\mathrm{atm}}^{2}$. The CHOOZ experiment in France looks for the disappearance of electron antineutrinos emitted from the nearby nuclear reactors. This experiment observed no disappearance and set a limit of

$$
\sin ^{2}\left(2 \theta_{13}\right)<0.15(90 \% \text { C.L. })
$$

More recently, MINOS, the T2K experiment in Japan and the Double Chooz experiment in France also reported results for their $\theta_{13}$ search. Preliminary reports of their combined findings hint at a non-zero $\theta_{13}[55]$ but further verification is needed.

The parameter $\delta_{\mathrm{CP}}$ is completely unconstrained since its determination relies on a definitive measurement of $\theta_{13}$. This is an important question for physics because a non-zero phase means neutrinos are CP-violating and may have played a role in the matter-antimatter asymmetry in the early universe via leptogenesis [56]. Several next-generation neutrino experiments are searching to shed light on the value of $\theta_{13}[57,58,59,60,61]$ and it is reasonable to expect our knowledge of neutrino mixing will greatly improve in the next decade.

\subsubsection{Non-standard Interactions}

While neutrino mixing is independent of interactions with matter, our experiments are built on Earth and neutrinos often travel through large distances in matter. It is established that neutrino oscillation takes place and explains the majority of observations relating to flavor change. Non-standard interactions, if they occur, will complement the model of neutrino 
mixing and oscillation. NSI models are purely phenomenological, currently with no established theory asserting the physics that gives rise to them. It is therefore essential to search for NSI in a model independent manner; the parametrization in Section 1.6 satisfies this.

Much like oscillations, there is not one experiment that can search for NSI in all sectors. Multiple experiments contribute to the constraints on $\varepsilon_{\alpha \beta}$ for each $\alpha, \beta$. The interpretation of these reports depends on the matter through which neutrinos traveled and which particles in said matter were included in the analysis. Baselines of experiments range from a few hundred meters to the Earth's diameter. For neutrinos traveling through the Earth's mantle and core as well as the crust, density variations are significant and MSW resonances in the denser mantle and core may become non-negligible. Through the MINOS baseline neutrinos remain in the crust, whose average density is taken to be $\rho=2.72 \mathrm{~g} / \mathrm{cm}^{3}$, giving $V_{e}=1.1 \times 10^{-13} \mathrm{eV}$.

An analysis can search for NSI parameters, $\varepsilon$, corresponding to interactions with $u, d$ quarks and electrons separately, or reduce them to the effective parameters which are summed over the contributions of the three fermions present in regular matter. When comparing results from two methods they need to be scaled accordingly [62]. In this dissertation we treat $\varepsilon$ as the effective NSI parameter summed over electrons, and up and down quarks (see Sec. 1.6), though down quarks dominate because of the larger interaction cross section and the work presented here is comparable to those experiments that make the assumption that only $d$-quarks contribute to NSI.

A comprehensive and inclusive analysis [33] gives the following bounds on all neutralcurrent NSI through Earth matter:

$$
\left|\varepsilon_{\alpha \beta}\right| \leq\left(\begin{array}{ccc}
4.2 & 0.33 & 3.0 \\
0.33 & 0.068 & 0.33 \\
3.0 & 0.33 & 21
\end{array}\right)
$$

where $\varepsilon_{\mu \tau}<0.33$ is the parameter MINOS is measuring. Eq. (1.92) is a reliable order-ofmagnitude estimate of the knowledge of upper limits for non-standard interactions. There are reports from single experiments or global fits that make more stringent claims with a variety of assumptions $[63,64]$; most recent and most similar to the analysis presented in this thesis is a measurement the Super-K experiment reported of a search for non-standard interactions. 
In contrast to MINOS, which is sensitive to NSI primarily through the sign change of the matter potential, $V$, due to its ability to distinguish neutrinos and antineutrinos, Super-K cannot identify the charge of the muon but is sensitive to NSI because of its much wider range of $L / E$. The two experiments' NSI analyses are therefore complementary. Super-K observes no non-standard interactions and sets the following limits on NSI parameters [65]:

$$
\begin{gathered}
\left|\varepsilon_{\mu \tau}\right|<1.1 \times 10^{-2} \\
-4.9 \times 10^{-2}<\varepsilon_{\tau \tau}-\varepsilon_{\mu \mu}<4.9 \times 10^{-2}
\end{gathered}
$$

Most of the constraints on NSI parameters are comparable to MINOS's sensitivity to non-standard interactions. This work is a valuable contribution to understanding NSI since it comes from a single experiment where systematic uncertainties and experimental factors are well understood and controlled. 


\subsection{THE MINOS BEAM AND DETECTORS}

The MINOS Experiment is made up of the NuMI (Neutrinos at the Main Injector) high intensity neutrino beam and two nearly identical tracking calorimeters: The Near Detector at Fermilab and the Far Detector in the Soudan Mine in Northern Minnesota. Side and top views of the detectors' locations are shown in Fig. 2.1.
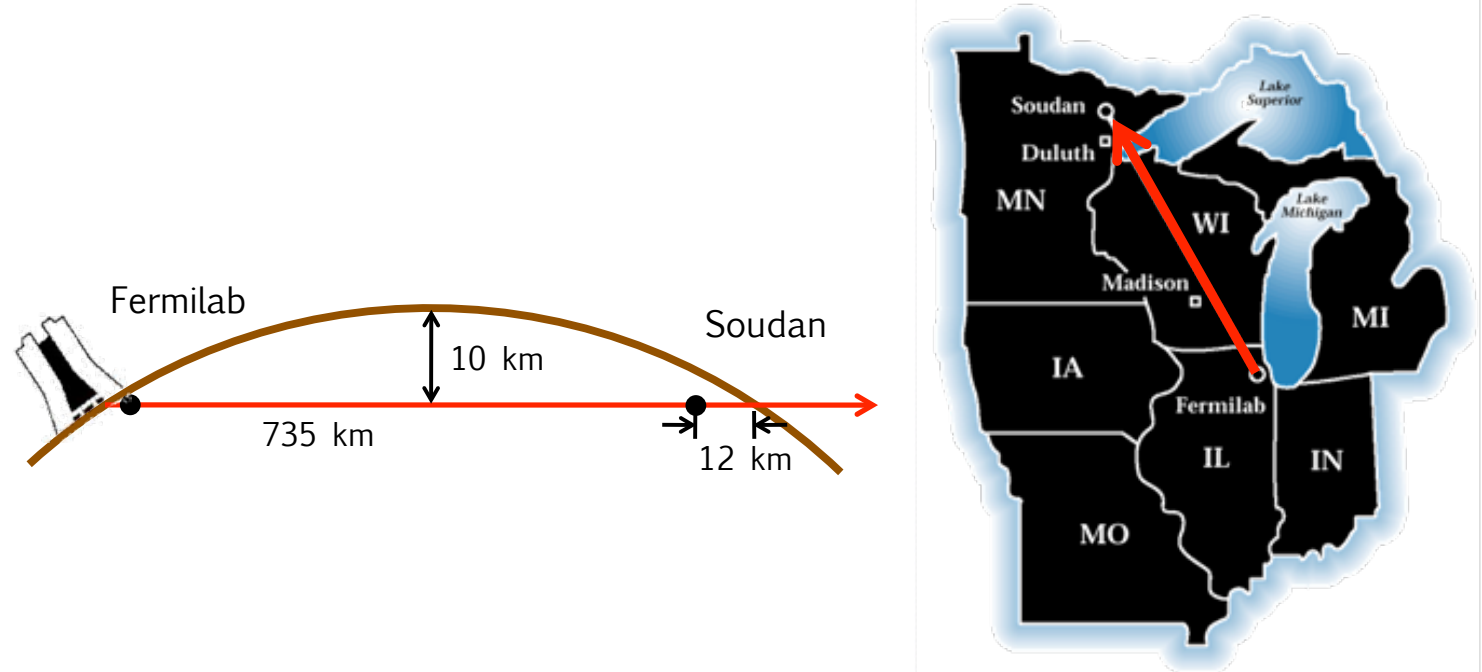

Figure 2.1: Side view (left) and bird's eye view diagrams of the locations of the MINOS detectors. Due to the Earth's curvature the beam is aimed downwards, travels through the crust to Soudan and exits the surface to the north of the Far Detector. The figure is adapted from $[66]$. 


\subsection{THE NUMI BEAM}

The NuMI beam, a schematic of which is shown in Fig. 2.2, is generated at Fermilab by using protons from the Main Injector (MI). The MI delivers $120 \mathrm{GeV}$ protons in a series of pulses, also called spills, each of which contains six batches. Of these six, NuMI receives five or six batches with the remaining one going to produce antiprotons for the Tevatron. A spill of protons arrives on average every 2.2 seconds and lasts for $10 \mu \mathrm{s}$. The typical spill intensity is $36 \times 10^{12}$ protons per pulse (ppp) when the Tevatron is also using the MI, and upwards of $40 \times 10^{12} \mathrm{ppp}$ when the accelerator runs in NuMI-only mode. The proton beam is aimed $58 \mathrm{mrad}$ downwards into the Earth by quadrupole and bending magnets to point at the Far Detector, which resides in an underground mine. The downward angle is necessary because of the curvature of the Earth. After passing through a series of beam monitoring devices, the proton beam reaches a baffle just upstream of the NuMI target. The baffle, a 150 $\mathrm{cm}$-long rod with a central aperture of radius $11 \mathrm{~mm}$, protects the downstream components by reducing the intensity of poorly focused protons.

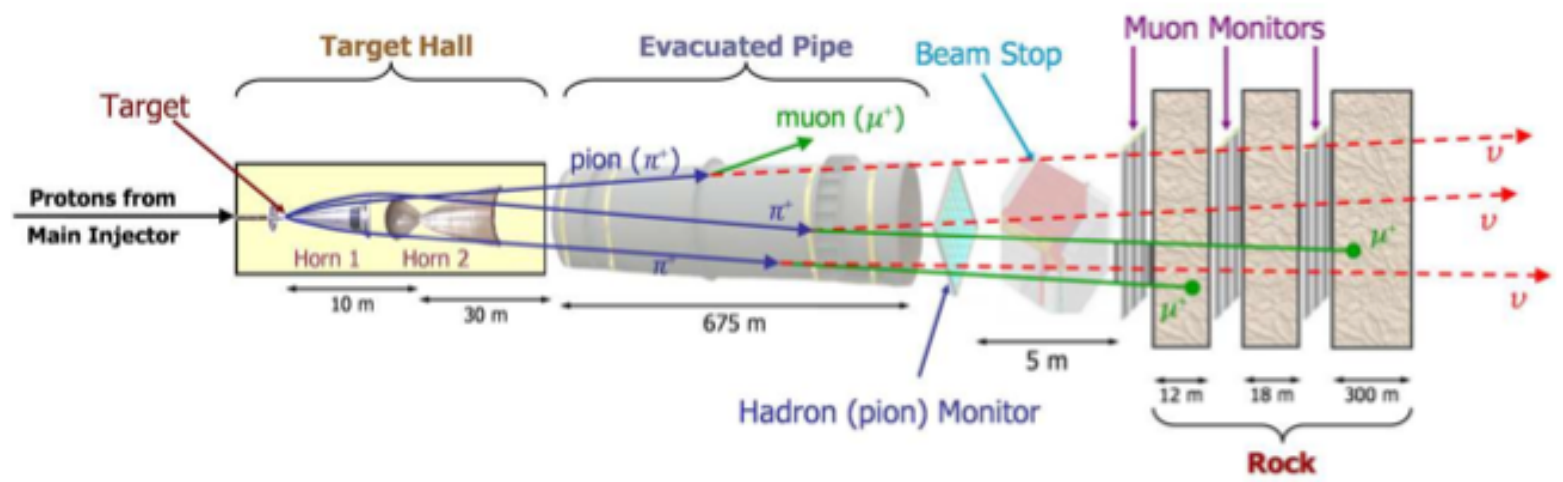

Figure 2.2: A schematic of the NuMI beamline. Protons from the Main Injector strike the graphite target; particles produced from this are focused by a two magnetic horns into the decay pipe where mesons decay into muons and muons neutrinos. Muons and undecayed hadrons range out through a series of absorbers and monitors; the neutrino beam continues to the detectors. Figure taken from [67]. 
The protons then strike a segmented, water-cooled, graphite target. The target consists of 47 segments of $6.4 \mathrm{~mm}$ width, $15 \mathrm{~m}$ height and $20 \mathrm{~mm}$ length, with $0.3 \mathrm{~mm}$ spacing. This gives the target a total length of $954 \mathrm{~mm}$ which corresponds to 1.9 interaction lengths. The graphite is water cooled by pipes running at the top and bottom of each segment. Fig. 2.3 shows the target and its enclosure.

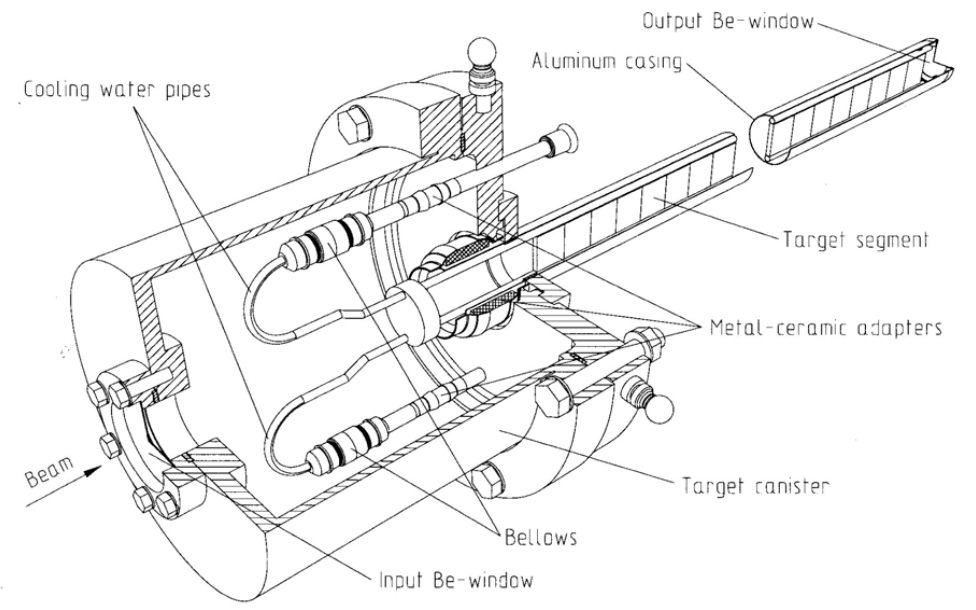

Figure 2.3: The NuMI target casing and components. The proton beam enters through the window in the target canister shown on the left hand side. The target is segmented as shown (not to scale) and cooled by two water pipes on either side. The segments are contained in an aluminum casing. Taken from [66].

The interaction of protons with the graphite target produces a variety of daughter particles including charged mesons (pions and kaons),

$$
p+C \rightarrow \pi^{ \pm}, K^{ \pm}+X
$$

These particles are focused by two magnetic horns that produce a toroidal magnetic field about an axis along the beam direction. The parabolic shape of the inner conductor makes the magnetic field act as a thin lens with focal length proportional to the meson's momentum, allowing momentum-selective focusing. The target is mounted at the upstream end of the first horn, sitting inside it. Most mesons are traveling parallel to the beam axis as they exit the horns. 
The decay pipe is a $675 \mathrm{~m}$ long steel pipe with a diameter of 2 meters where the mesons decay into muons and muon neutrinos:

$$
\begin{aligned}
\pi^{+(-)} & \rightarrow \nu_{\mu}\left(\bar{\nu}_{\mu}\right)+\mu^{+(-)}, \\
K^{+(-)} & \rightarrow \nu_{\mu}\left(\bar{\nu}_{\mu}\right)+\mu^{+(-)} .
\end{aligned}
$$

The decay pipe was evacuated to down to about 1 Torr for the first two runs of the experiment. Data from subsequent runs were collected with the decay pipe filled with Helium gas at $0.9 \mathrm{~atm}$. The decision to fill the decay pipe with Helium gas was taken in order to prevent a potential implosion which became a significant risk. The presence of He in the decay pipe results in a slight decrease in the focusing peak as some mesons interact with the gas before they can decay. It also acts as an additional meson production target resulting in a small increase in the flux above the focusing peak.

The beam goes through a hadron absorber following the decay pipe where undecayed mesons and uninteracted protons get absorbed. The muons range out to 240 meters of rock after the absorber where they, too, get stopped. There is one hadron monitor directly upstream of the hadron absorber and three muon monitors, placed in the rock that follows the absorber, alternating with layers of rock. The beam then reaches the Near Detector. Muons from interactions with the rock just upstream of the detector also enter through the detector face. In this forward horn current (FHC) focusing mode the beam detected by the Near Detector is composed of $91.7 \% \nu_{\mu}, 7 \% \bar{\nu}_{\mu}$ and $1.3 \%\left(\nu_{e}+\bar{\nu}_{e}\right)$. The antineutrino component comes from negative mesons traveling through the center of the horns that do not get deflected. The electron flavor component is due to secondary decays of muons. The Near Detector spectra of muon neutrinos and antineutrinos in FHC-mode are shown in Fig. 2.5 .

In order to make an antineutrino beam, the polarity of the horn current is reversed to focus negatively charged mesons which decay into antineutrinos. Cartoons of forward horn current (FHC) neutrino-mode and reversed horn current (RHC) antineutrino-mode focusing are shown in Fig. 2.4. The composition of the reversed horn current (RHC) beam integrated over all energies is $40 \% \bar{\nu}_{\mu}, 58 \% \nu_{\mu}$ and $2 \%$ electron flavored. The spectra in this mode are 
shown in Fig.2.6.
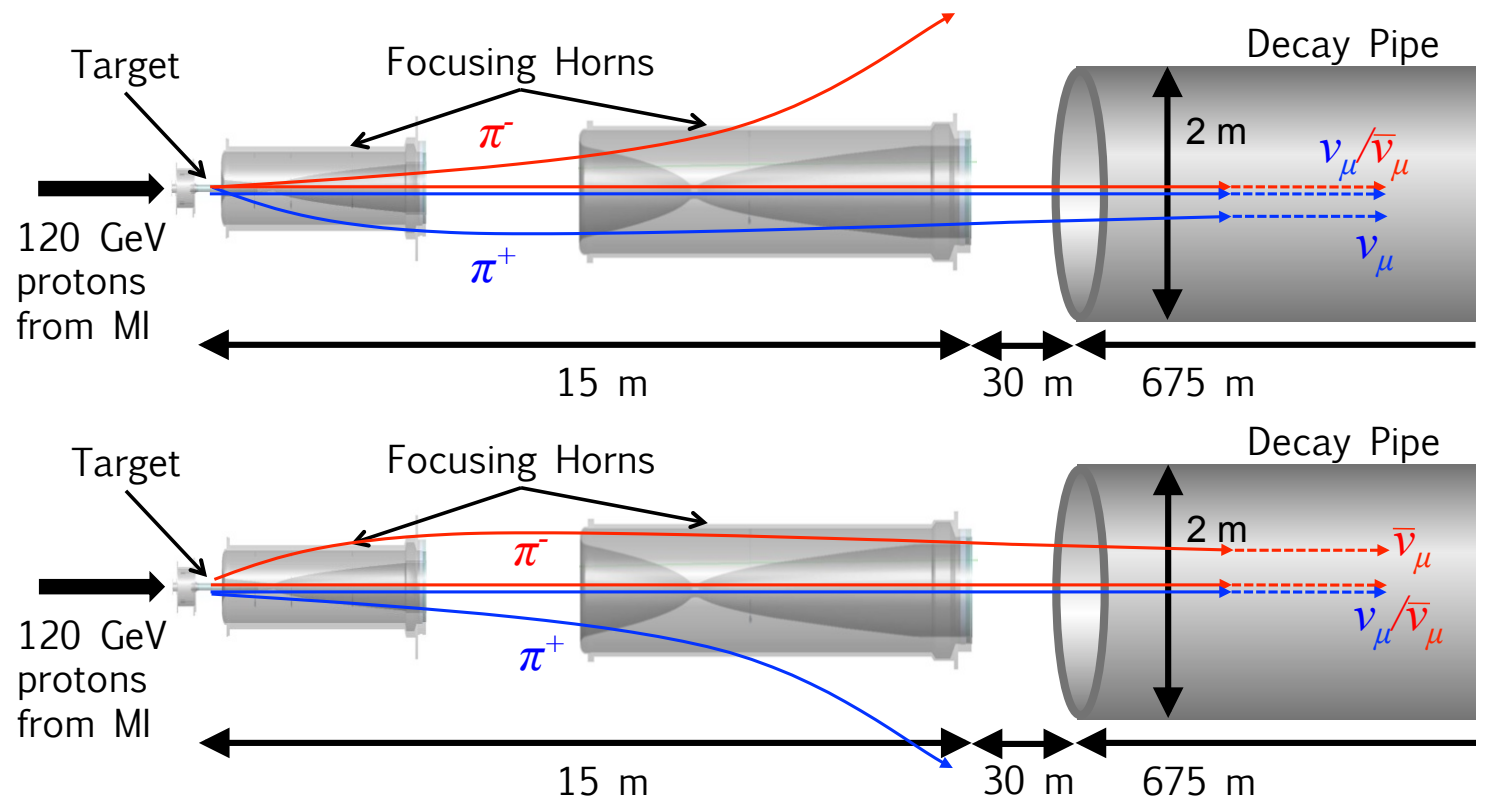

Figure 2.4: The sketches show the upstream part of the beamline geometry and focusing optics in forward (top) and reversed horn current modes. Figures taken from [68]. 


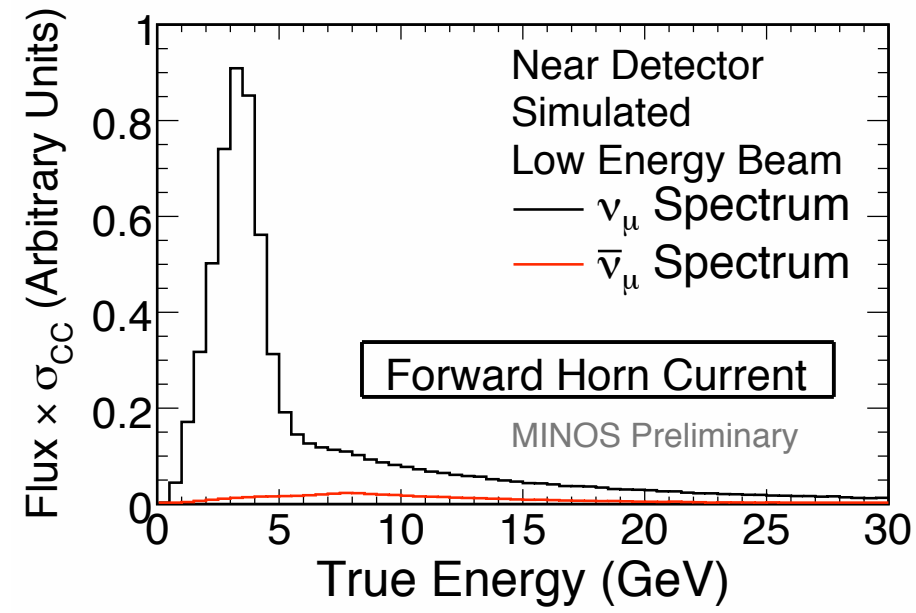

Figure 2.5: Simulated Near Detector energy spectra of muon neutrinos and antineutrinos in forward horn current mode.

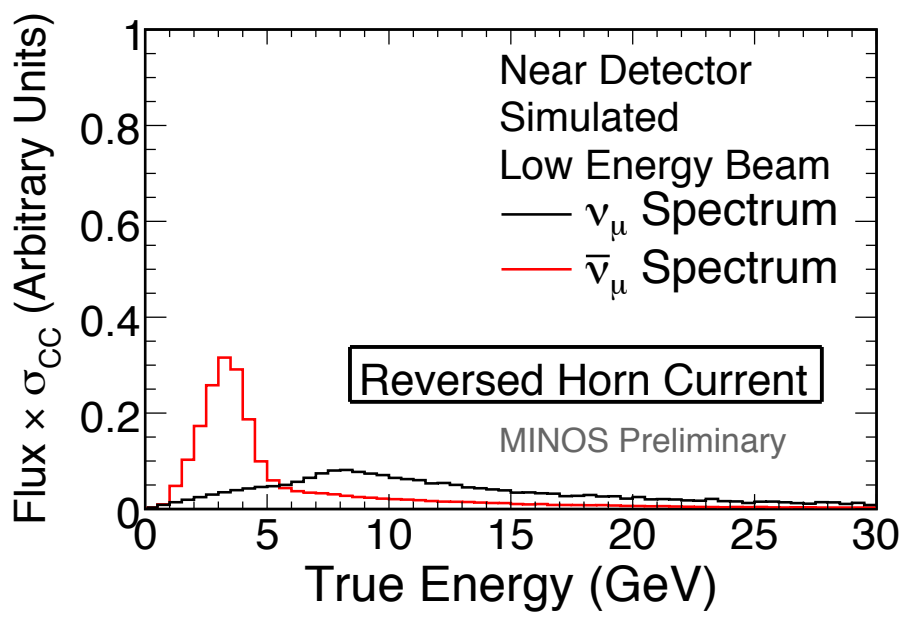

Figure 2.6: Simulated Near Detector energy spectra of muon neutrinos and antineutrinos in reversed horn current mode. 
Reversing the horn polarity changes the spectra of signal and background because of the combination of two effects; first the beam is produced by protons which results in a larger flux of positively charged mesons and second, the interaction cross section of antineutrinos with the Near Detector is about half that of neutrinos [69, 70]. This results in a smaller flux compared to neutrino-mode.

The NuMI beamline can be operated in multiple configurations. The peak energy of the neutrino beam can be adjusted by varying the target position relative to the horns. Fig. 2.7 shows the three configurations that result in low, medium and high energy beams. (All of the data used for physics analysis in this thesis was collected in the "LE-10" configuration where the separation between the horns is $10 \mathrm{~m}$ and the horn current is $185 \mathrm{kA}$.)
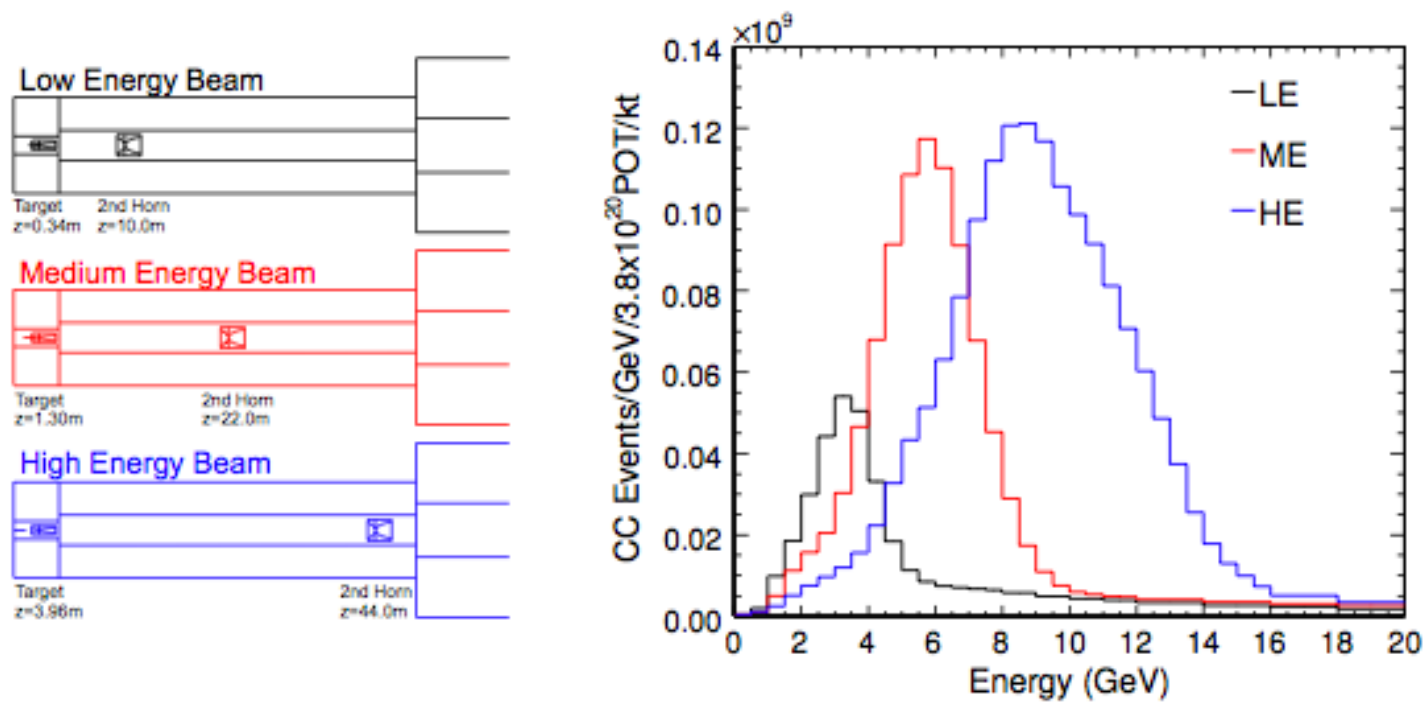

Figure 2.7: Three NuMI beam configurations used in MINOS and the resulting energy spectra at the Near Detector. Data presented in this thesis was collected in the low energy configuration (black curve). 


\subsection{THE MINOS DETECTORS}

The two detectors are functionally equivalent. They are designed to be as similar as possible to minimize the systematic uncertainty arising from detector effects. They are both magnetized steel and scintillator tracking calorimeters with alternating views of scintillator. A schematic of the steel and scintillator planes is shown in Fig. 2.8. Both detectors are made up of alternating layers of $2.54 \mathrm{~cm}$ thick steel and $1 \mathrm{~cm}$ thick scintillator strips of 4.1 $\mathrm{cm}$ width. The scintillator strips are aligned perpendicular to one another at \pm 45 degrees in $U$ and $V$ views to allow three dimensional reconstruction. The Near and Far Detector scintillator module arrangements are shown in figures 2.9 and 2.10 .
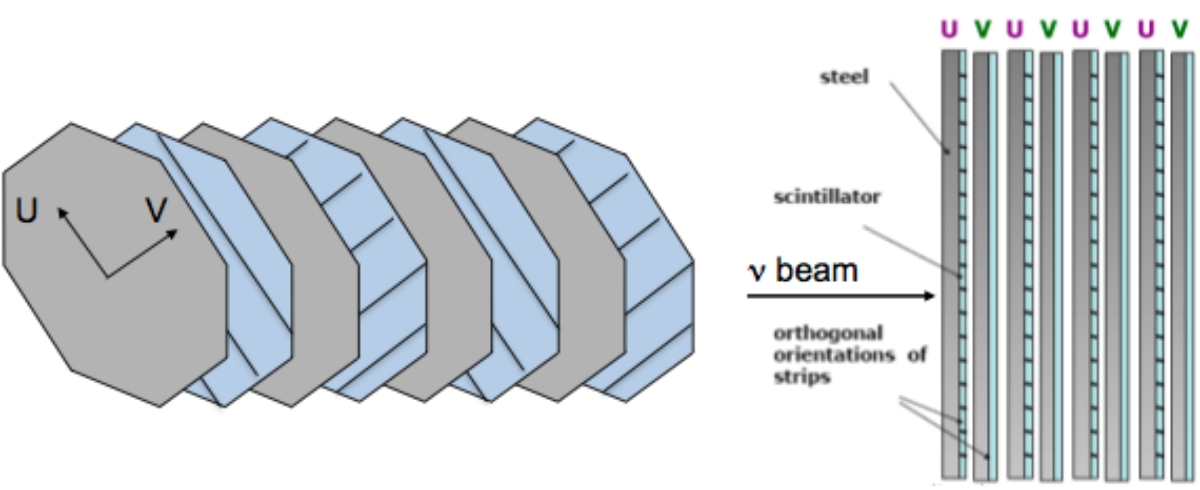

Figure 2.8: A diagram of MINOS detector planes and scintillator. Alternating layers of scintillator are perpendicular to each other at \pm 45 degrees to allow $3 \mathrm{D}$ reconstruction, the axes are labeled $U$ and $V$ views. The right diagram shows the side view of the layers and beam direction. From [68]. 

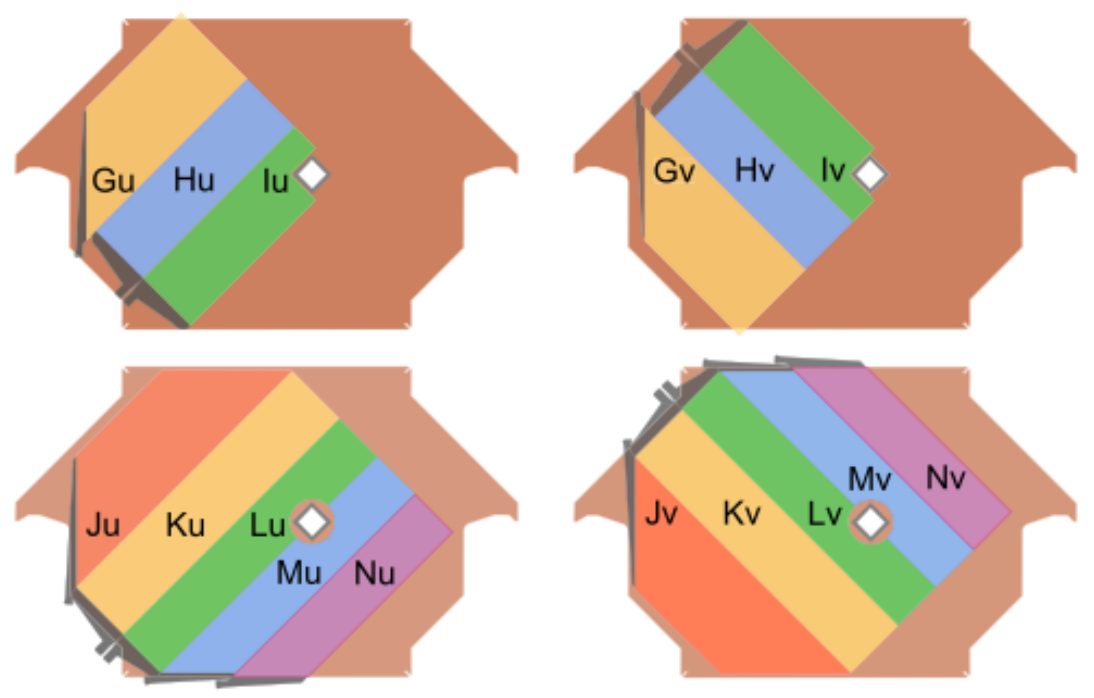

Figure 2.9: The arrangement of partially (top) and fully instrumented scintillator modules on Near Detector planes. The small letters $u$ and $v$ denote the orientation of the strips while the capital letters denote different module geometries. The off-center diamond marks the position of the coil hole. Figure taken from [71].
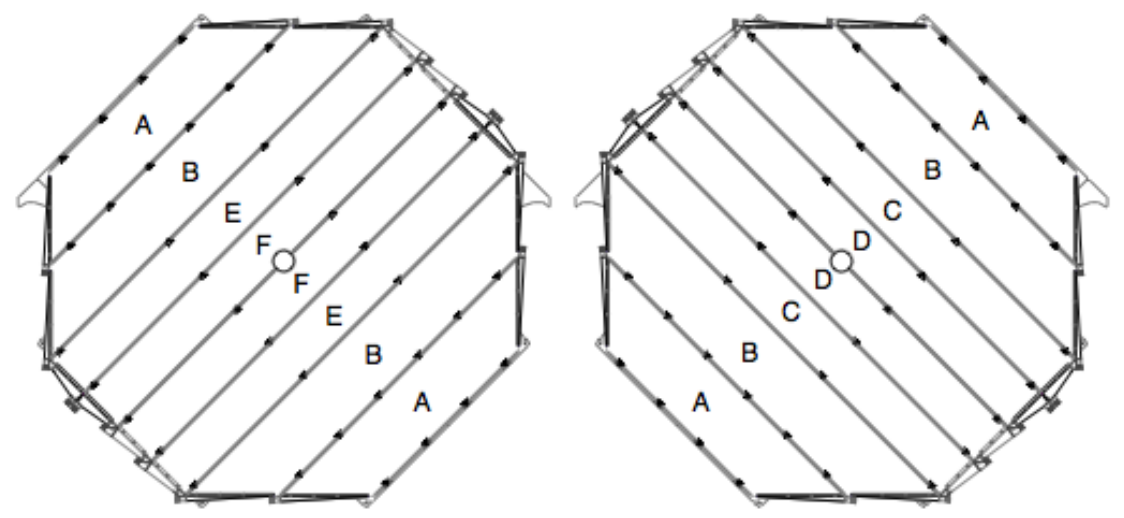

Figure 2.10: The arrangement of scintillator modules on Far Detector planes, a U-view plane on the left and a V-view plane on the right. The modules labeled A and B comprise 28 scintillator strips, the narrower C, D, E and F modules have 20. Figure taken from [71]. 


\subsubsection{Near Detector}

The Near Detector (ND), located $110 \mathrm{~m}$ underground at Fermilab, is a $4.8 \mathrm{~m}$ wide, $3.8 \mathrm{~m}$ high squashed octagon with a length of $16.6 \mathrm{~m}$. It consists of 282 steel planes and has a mass of 980 tons. The first 120 planes are instrumented with scintillator, every fifth one fully and the other four partially (see Fig. 2.9). The remainder of the detector has every fifth plane fully covered with scintillator where intermediate four planes have no scintillator. See Fig. 2.11 for the cross section of a ND plane.

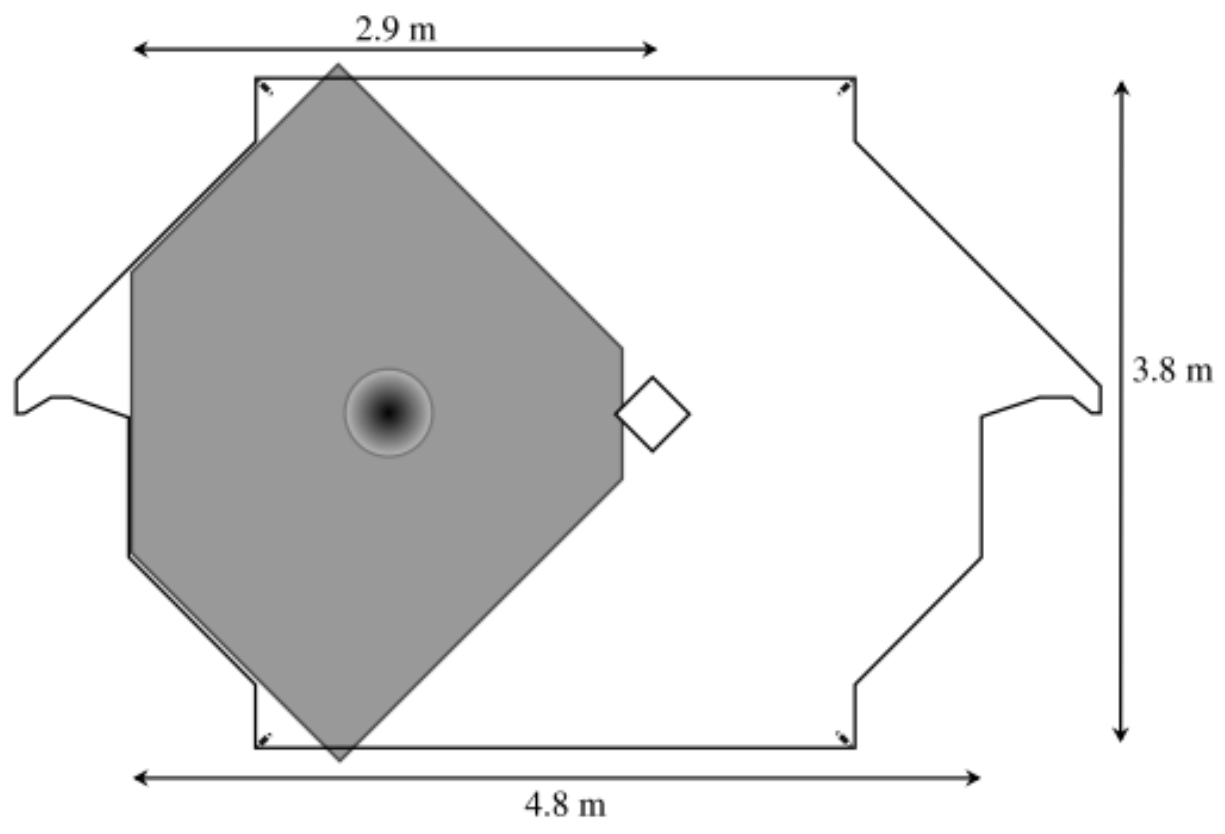

Figure 2.11: The cross section of a MINOS ND plane. The dark grey region indicates the instrumented region of a partially instrumented plane. Fully instrumented planes have the entire area covered with scintillator. The diamond in the center is the coil hole and the gray dot on the left hand side denotes the center of the neutrino beam. From [69].

The upstream region of the detector is more finely instrumented and serves as the calorimeter. The calorimeter is designed to measure hadronic showers while the partially instrumented second half of the detector, the spectrometer, is for muon tracking. The detector is magnetized to an average magnetic field strength of $1.3 \mathrm{~T}$ which focusing particles 
of one charge sign and defocusing the other. The magnetic field allows the reconstruction of the charge of a particle traveling through the detector; the curvature of a charged particle is also used to reconstruct its momentum. The beam axis is horizontally approximately 1.5 $\mathrm{m}$ away from the coil in an attempt to minimize the number of tracks crossing the coil hole region.

\subsubsection{Far Detector}

The Far Detector has an octagonal cross section 8m across, a length of $31 \mathrm{~m}$ and a mass of 5.4 kton. It is made up of a total of 486 steel planes in two supermodules of 243 planes each. The supermodules are separated by a gap of $1.2 \mathrm{~m}$ length. The event rate at the Far Detector is of the order of a few events per day, in contrast to approximately ten interactions per spill at the Near Detector. This is the reason for the detector's larger size. It is deeper underground than the Near Detector, at $2341 \mathrm{ft}$ depth, to reduce the cosmic ray background. Cosmic rays are also vetoed by a veto shield covering the top of the detector along its length. The detector is uniformly instrumented where every plane has full coverage with 192 strips of scintillator, except for the front planes of each supermodule. A magnetic coil runs down the center and magnetizes the detector to an average magnetic field of $1.3 \mathrm{~T}$ strength, the same as the Near Detector. 


\subsection{SCINTILLATOR}

Each scintillator strip is made of polystyrene doped with scintillator fluors PPO and POPOP and covered in a reflective coating of $85 \%$ polystyrene and $15 \% \mathrm{TiO}_{2}$ by weight. A charged particle traveling through the strip excites the fluor, which emits the absorbed energy in ultra violet photons. Wavelength shifting (WLS) fibers, $1.2 \mathrm{~mm}$ in diameter, are embedded in a $2 \mathrm{~mm}$ deep groove in the middle of each strip and run along the length of the strip. See Fig. 2.12 for a schematic of the scintillator system. These WLS fibers absorb the UV scintillation light and emit it in green (average wavelength of $530 \mathrm{~nm}$ ) and transport it to the end of the strip. The scintillator strips are grouped into modules of 13 to 28 strips and enclosed in a $0.5 \mathrm{~cm}$ thick aluminum casing.

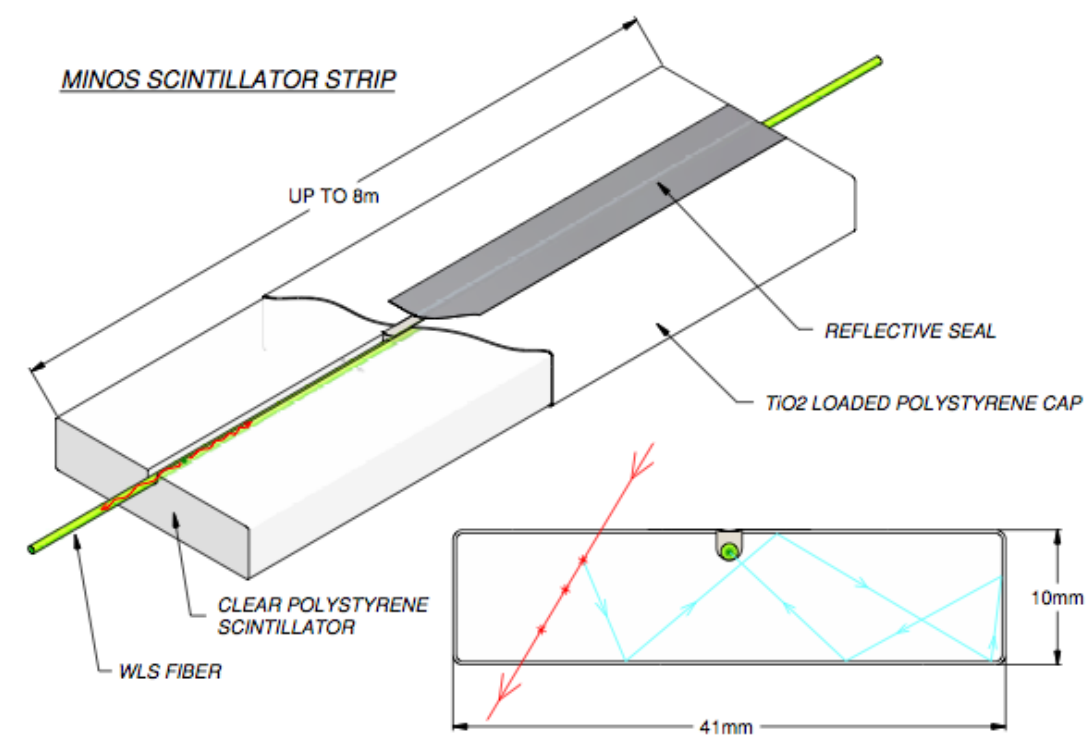

Figure 2.12: Cross sectional view of a MINOS scintillator strip, from [71]. 


\subsection{PHOTOMULTIPLIER TUBES}

The Near Detector is read out by Hamamatsu 64-anode (M64) PMTs each anode of which acts as a single-anode phototube. The light from scintillator strips is carried by WLS fibers which are bundled into an optical connector and fed to an optical cable. This cable carries the signal into the MUX box. Inside the box, fibers carry the light onto the face of the PMT. Fig. 2.13 shows the readout of scintillator strips by photomultiplier tubes.

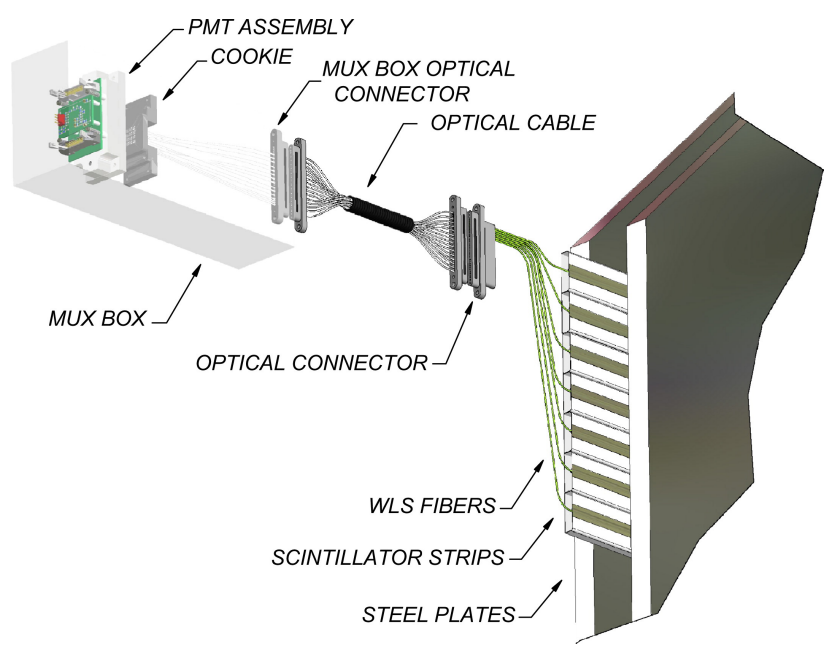

Figure 2.13: The readout chain starting from WLS fibers leaving the scintillator strips, going through optical fiber and connectors and arriving at the PMTs. Taken from [71].

The incident light strikes the photocathode of a tube releasing photoelectrons. These photoelectrons travel through a series of dynodes at a positive voltage with respect to the photocathode. The number of electrons gets multiplied at each dynode through secondary emission; the anode at the end of the tube finally records the sharp pulse caused by the accumulation of charge. The photoelectrons are multiplied through 12 dynodes and the average voltage between the cathode and the anode is $800 \mathrm{~V}$. The typical gain, the ratio of the number of electrons at the anode to that at the cathode, is $1 \times 10^{6}$ with a linear response for input pulses of up to 50 photoelectrons. The quantum efficiency (QE), i.e. the probability of a photon releasing a photoelectron is about $12 \%$ for a photon of $520 \mathrm{~nm}$ wavelength. The PMTs are housed in steel, light-tight boxes, which keep the tubes dark, shield them from 
the magnetic field and shield the electronics from ambient electronic noise.

Each scintillator strip in the first 120 planes of the Near Detector, the calorimeter, is read out individually: One strip connects to one PMT whose anode is read out by a unique electronic channel. In the spectrometer there is 4:1 multiplexing, where four anodes are connected in parallel and read out by one electronic channel in order to reduce the amount of front-end electronics necessary. The four multiplexed strips are $1 \mathrm{~m}$ apart making the muon tracking unambiguous.

In the Far Detector 16-anode Hamamatsu M16 PMTs are used. The multiplexing at the Far Detector is such that eight fibers (from eight scintillator strips) are fed to one pixel in a PMT. The strips from which these fibers carry light are at least one meter apart and result in unambiguous track reconstruction. The Far Detector PMTs have gains of $1 \times 10^{6}$, linear up to 100 photoelectrons and greater than $12 \%$ quantum efficiency.

A low pulse height hit (usually of less than one photoelectron) can be registered at a nearest neighbor of an anode. This phenomenon is referred to as cross talk and is an artifact of the multi-anode design of the phototubes; charge drifts from one anode to another. Total cross talk in all pixels is 3.7\% in Near Detector PMTs and 3.2\% in Far Detector PMTs.

\subsection{THE MAGNETIC FIELD}

Both MINOS detectors are magnetized by a coil that passes through a hole cut through the length of the detector. The Far Detector coil hole is at the center of the octagon and each supermodule is magnetized independently by its own coil. The coil consists of 190 turns of copper wire with Teflon insulation water-cooled by a copper jacket. Fig. 2.14 shows the cross section of a Far Detector coil. An 80 A power supply gives a $15.2 \mathrm{kA}$-turn total current providing an average toroidal magnetic field of $1.3 \mathrm{~T}$ strength [71]. One coil dissipates 20 $\mathrm{kW}$ of power.

In the Near Detector the coil hole is offset about $1.5 \mathrm{~m}$ to the right of the beam center so that the fiducial volume excludes the coil hole region. This minimizes the number of tracks that travel through the uninstrumented coil hole region and get reconstructed poorly. Due 


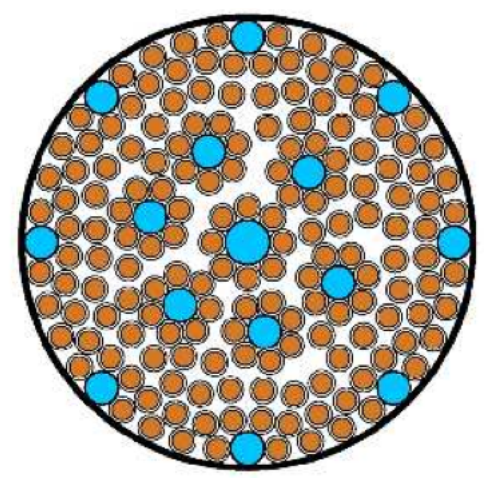

Figure 2.14: Cross section of a Far Detector coil. The larger circles represent the copper cooling tubes and the smaller circles are the 190 turns of copper wire. From [71].

to the squashed octagon geometry, a 40kA-turn current is required [71]. The ND coil, whose conductor is made of aluminum, is also water-cooled and has eight turns. It dissipates a power of $47 \mathrm{~kW}$. The magnetic field in both detectors' fiducial volumes are nearly uniform. Field maps of both detectors can be seen in Fig. 2.15.
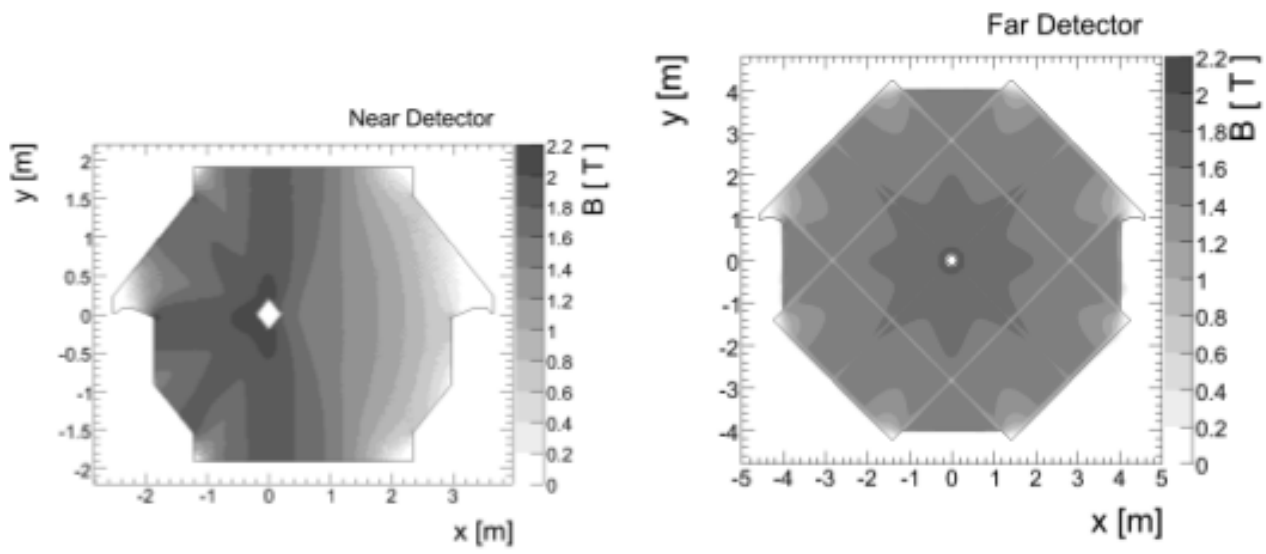

Figure 2.15: The left hand figure shows the Near Detector magnetic field map where offcenter diamond marks the position of the coil hole. On the right is the Far Detector field map with the rectangular coil hole in the center. From [71]. 


\subsection{ELECTRONICS AND DATA ACQUISITION}

The Near and Far Detectors were designed to be as similar as possible to minimize the effect of systematic uncertainties. The same electronics, however, could not be used for both detectors. In the Far Detector the beam neutrino interaction rate is on the order of a few per day, whereas the cosmic muon rate is around $0.5 \mathrm{~Hz}$. By contrast, the Near Detector sees on the order of 10 neutrino interaction events per every $10 \mu$ s spill. The following two sections describe the Near and Far Detector front end electronics, followed by a section on the data acquisition (DAQ) system which is common to the two detectors.

\subsubsection{Near Detector Front-End Electronics}

In order to resolve the amount of data it collects, Near Detector electronics are designed to be fast and have no deadtime $[71,72]$. The readout has a time resolution of 18.8 ns which matches the $53 \mathrm{MHz}$ frequency of the Main Injector RF cavities.

The signal from each PMT anode is fed into a QIE (Charge (Q) Current (I) Encoder), which consists of a current splitter, gated integrator and range selector as shown in Fig. 2.16. The current is split into eight binary weighted ranges with values $I / 2, I / 4, \ldots, I / 256$ and integrated onto a capacitor for each range. See Fig. 2.17 for a plot of the QIE response. The capacitors are sensitive to their respective ranges. This saturates the capacitors of all ranges below that corresponding to the signal size and the current in the first unsaturated range provides a precision measurement of the signal. Splitting the current gives the QIE chip sensitivity over a range of input charges, from $10 \mathrm{fC}$ to $10 \mathrm{pC}$.

After integration, the voltage across the capacitors is fed to an ADC (analog/digital converter) via a range selector. The voltage across one and only one of the capacitors is within the predetermined limits of the ADC, which is selected by the range selector and output to the ADC, along with a three bit number indicating the range value. The current splitter integrates all the current in one clock cycle, selects the unsaturated range in the next cycle. In the following clock cycle, the ADC converts the analog signal into an 8-bit digital signal. A fourth cycle is required to reset the capacitors. In order to have deadtimeless 
operation, there are four independent copies of the current splitter on the QIE. In addition to the 8-bit ADC value and the 3-bit range value, a 2-bit code (called CAPID) identifies the current splitter and capacitor from which the signal was read. This 13-bit output from the ADC is stored on a FIFO (first in first out) buffer which can store digital signal from up to 1000 clock cycles $(19 \mu \mathrm{s})$.

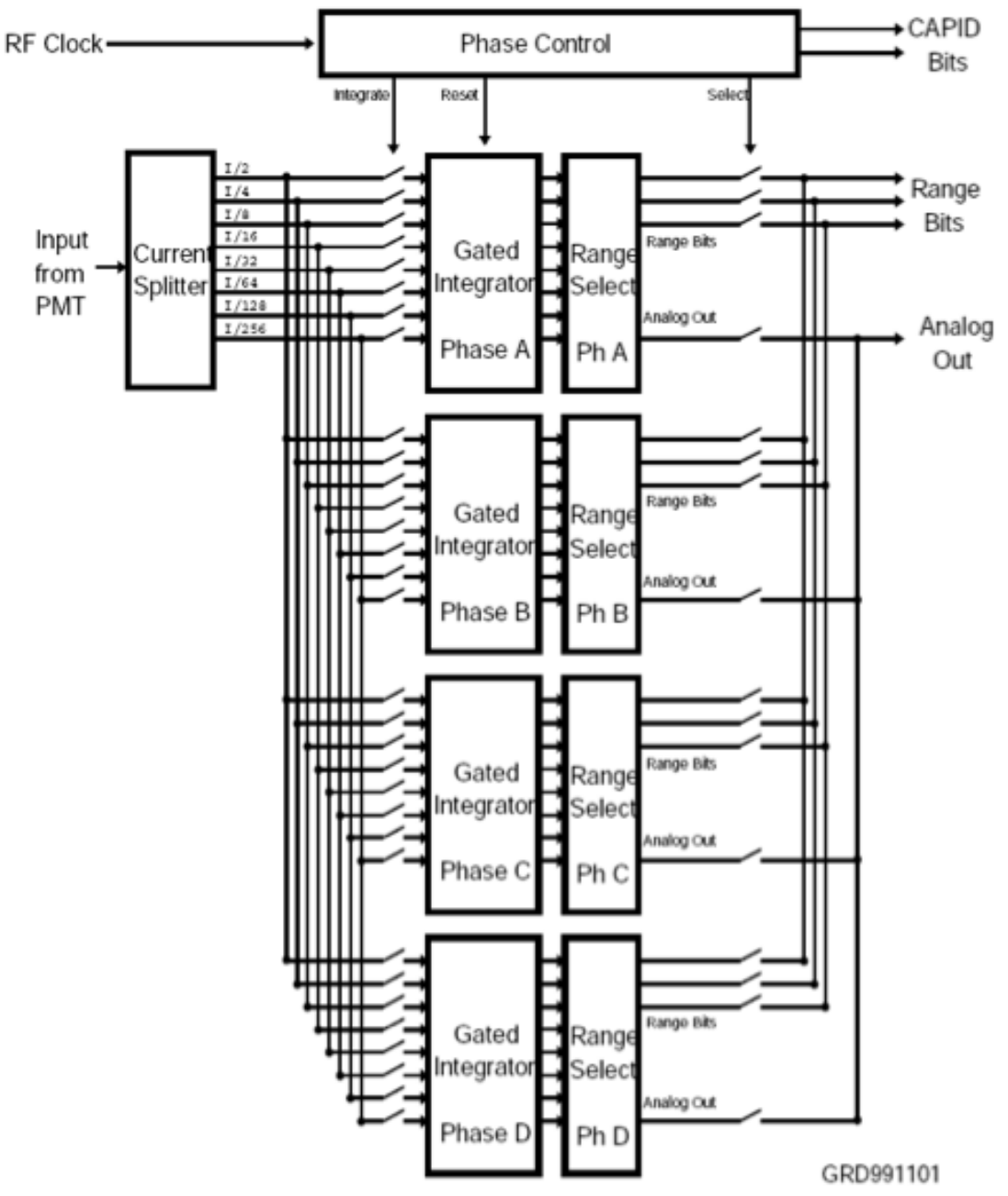

Figure 2.16: Block diagram of the QIE chip for the Near Detector. It has three main components, current splitter, gated integrator and range selector. The chip digitizes the analog signal from the PMT. From [72]. 


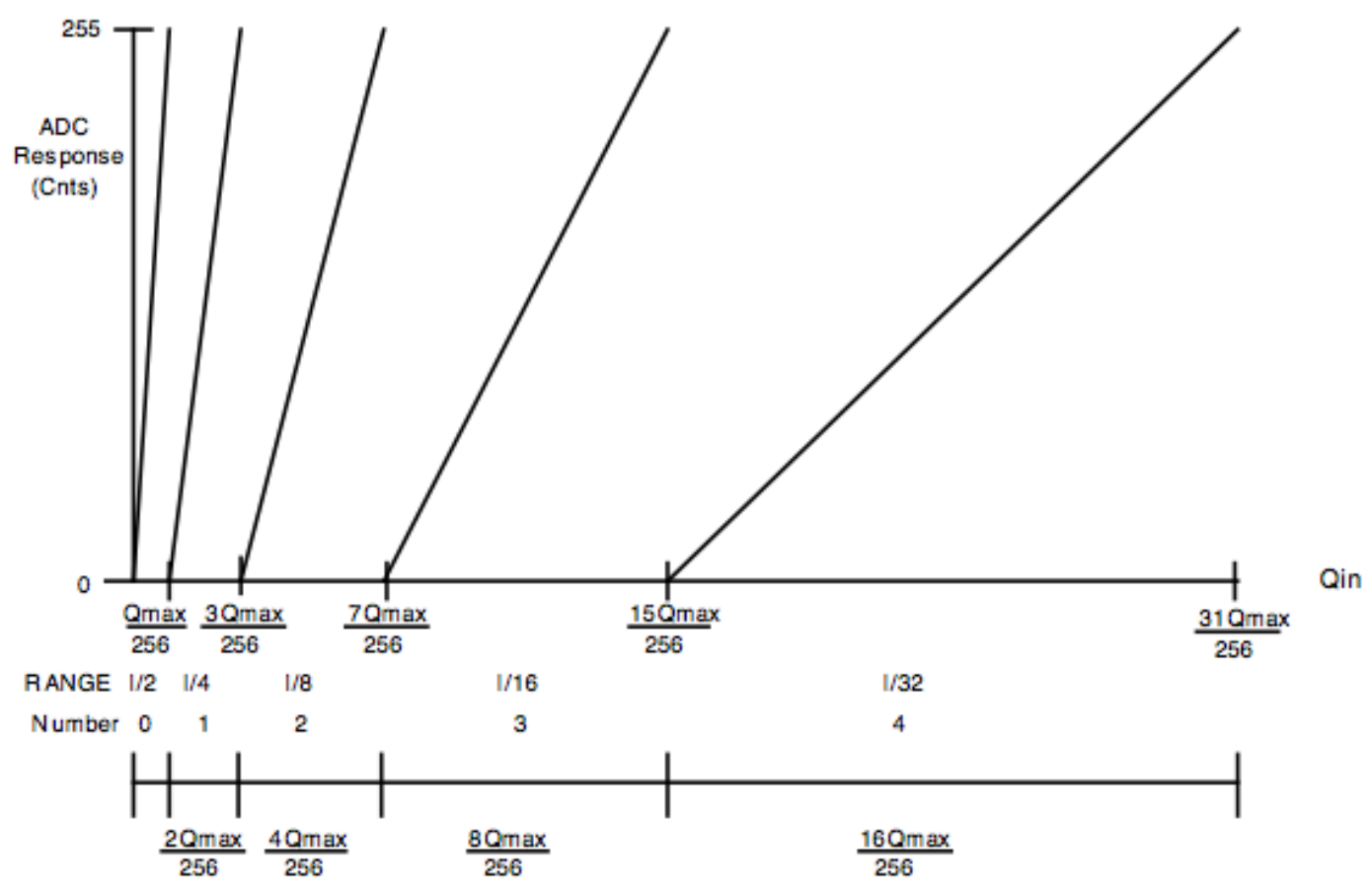

Figure 2.17: Response of the QIE chip. The $x$-axis shows the amount of charge injected into the chip, the $y$-axis shows the ADC response for the different ranges. Only the first five of the eight ranges are shown. From [71] 
The QIE is mounted on a MENU (MINOS Electronics for Neutrinos), the smallest frontend electronics unit. A MENU holds a QIE chip, an ADC and a FIFO along with a DC charge injector which can inject signal into the QIE for calibration as shown in Fig. 2.18.

\section{MENU Module}

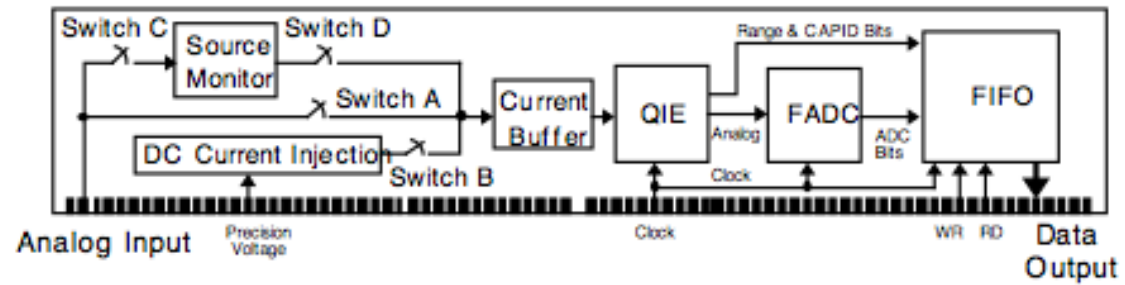

\section{MINDER Module}

Accommodates 16 MENU Modules.

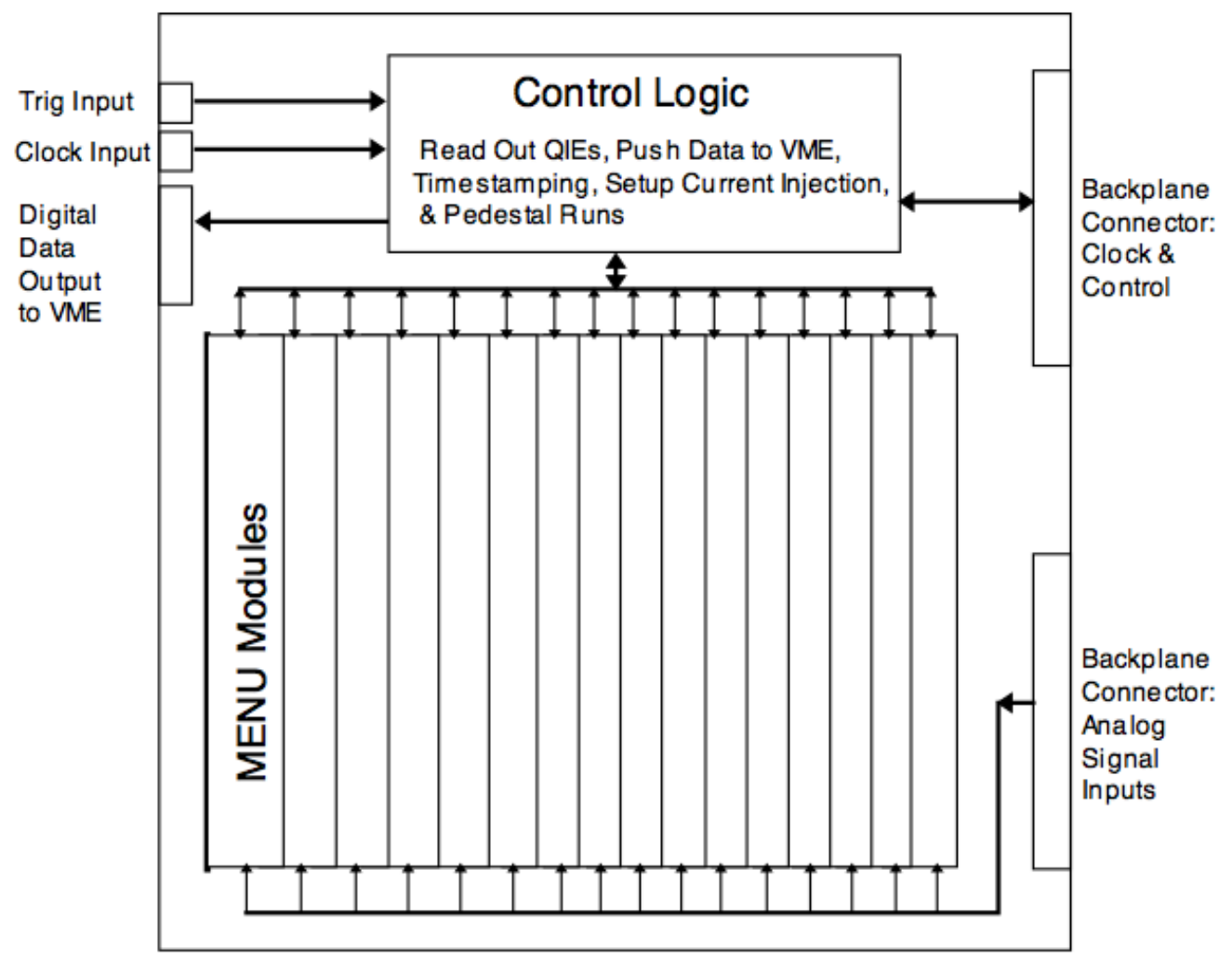

Figure 2.18: Block diagram of a MENU module (top) and a MINDER module, from [72] 
Sixteen MENU boards are mounted on a MINDER module (MINOS Near Detector Electronics Readout, fig 2.18). The MINDER timestamps the signal and controls the power to the MENUs. The MINDER reads all the output from its FIFOs and sends them to a single FIFO on the MASTER module (MINOS Acquisition, Sparsifier and Time-stamper for Event Readout). Eight MINDERS are connected to a MASTER which has two buffers, one is being written to while the other is being read by a VME computer. The VME computers send the data to the data acquisition system. The diagram in Fig. 2.19 provides a schematic overview of the ND front-end electronics readout.

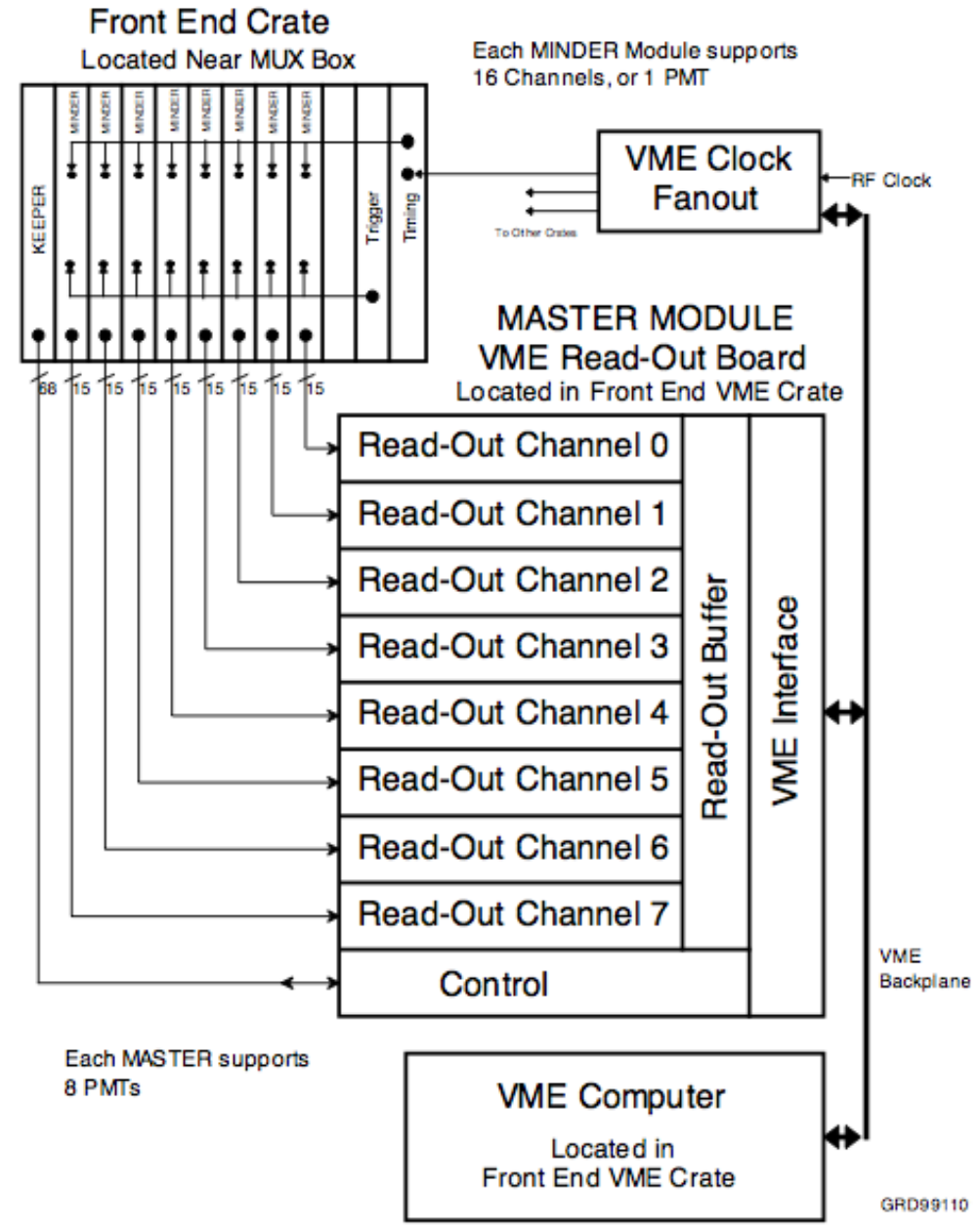

Figure 2.19: Overview of Near Detector front-end electronics, from [72] 


\subsubsection{Far Detector Front-End Electronics}

The data rate at the Far Detector, $735 \mathrm{~km}$ downstream of the neutrino source and $700 \mathrm{~m}$ underground, is much lower than that of the Near Detector: a few beam neutrino interactions per day and a $0.5 \mathrm{~Hz}$ cosmic muon rate. This means electronics can be slower than the Near Detector and deadtime can be tolerated. A diagram of the Far Detector front-end electronics is presented in fig. 2.20 .

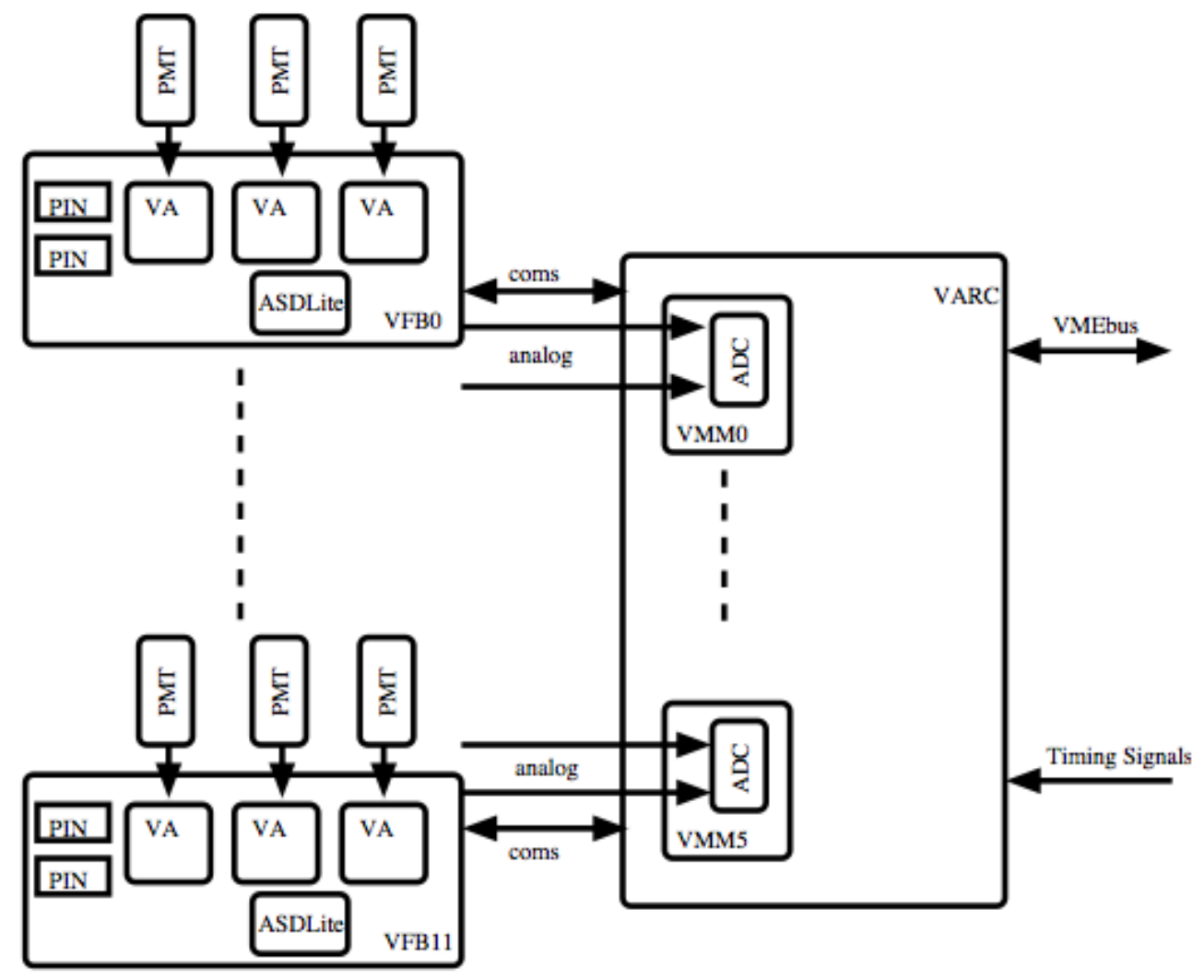

Figure 2.20: Overview of the MINOS Far Detector readout electronics. Diagram from [71].

Each PMT is read out by a Viking VA chip using 17 of its 32 channels. 16 channels are used to read out an anode each, and one channel reads out the PIN diode monitoring the light injection system, see Sec. 3.2.1.1. The VA chips are mounted on VA front-end boards (VFB) which provide power control and biasing for the VA chips. The VFB also houses a chip called ADSLite. This chip receives a signal from the PMT and triggers the VA readout if the signal is greater than 0.25 photoelectrons. 
VFBs feed and analog/digital converter which is housed on a VMM (VA Mezzanine Module); two VFBs are connected to one VMM. The VARC (VA Readout Controller) handles signal digitization, triggering, time-stamping and bias of the VA chips. The VFBs are fully controlled by the VARC. One VARC module reads 36 PMTs through 6 VMMs, each of which read 6 PMTs. The digitization of the VA chip takes $5 \mu \mathrm{s}$, meaning if all six need to be read out, all six chips would be dead to new signals for $30 \mu \mathrm{s}$. In order to alleviate the deadtime cost of reading out, at least two dynode signals within a $400 \mathrm{~ns}$ window are required to trigger readout.

The digitized data are sent to a FIFO, pedestal subtracted then written to an on-board VME memory, which is subsequently read out by the DAQ.

\subsubsection{Data Acquisition}

The Near and Far Detector data acquisition systems are functionally identical. A diagram of Far Detector DAQ components is shown in Fig. 2.21. The DAQ system is designed to read out the front-end electronics in a continuous manner and to store this data on a farm.

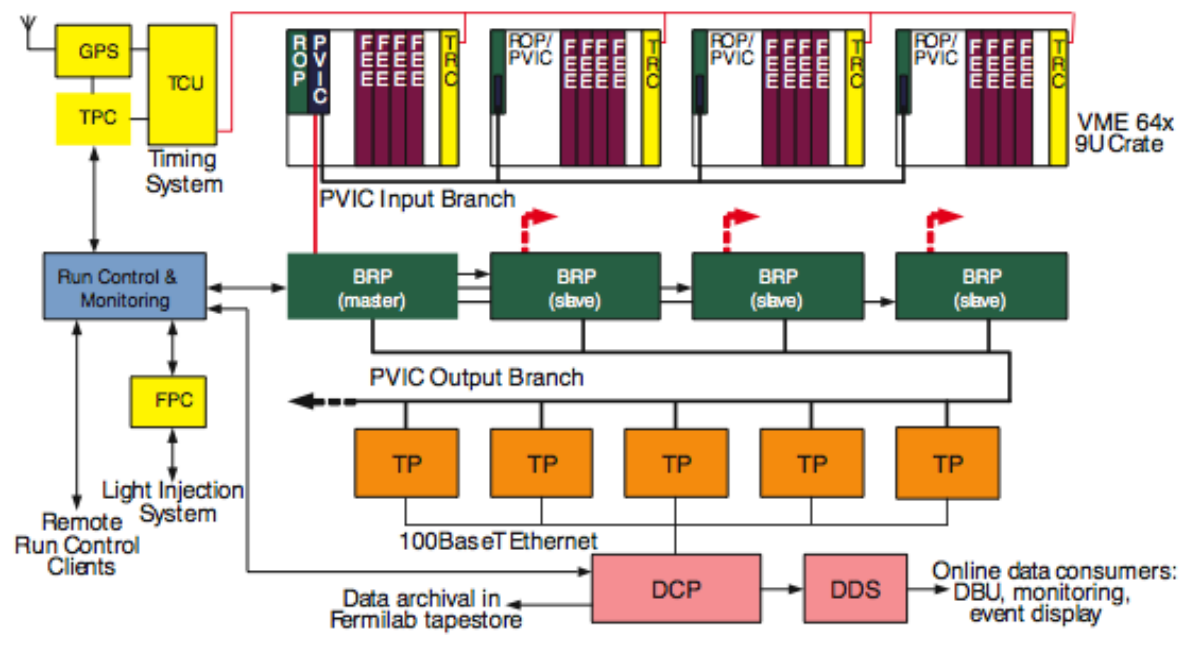

Figure 2.21: Diagram showing components of the MINOS data acquisition system. The Far Detector DAQ is shown here as an example, the Near Detector system functions identically. Image from [71]. 
The DAQ reads out data continuously but only writes it to disk under certain conditions. The Near Detector DAQ is triggered by the beam spill signal at Fermilab and all data arriving within a $100 \mu$ s window around the spill is recorded. Since the Far Detector is not at Fermilab, it cannot be triggered directly by the beam spill. Instead, it receives the GPS timestamp of the beam spill from the Near Detector via the Internet.

A MASTER has two buffers, as discussed in section 2.6.1, one of which is being written while the other is being read out, by a ROP (Readout Processor). The buffers are read for the duration of one timeblock, 10-50 ms long. The ROP sends all the data collected for one timeframe, 1 second in duration, to Branch Readout Processors (BRPs). The BRP then sends this data to the trigger farm. The trigger farm constructs events from the raw scintillator hit information and writes those that pass the triggering algorithm to disk. The trigger farm is also responsible for storing calibration information.

\subsection{CALIBRATION DETECTOR}

The calibration detector, or CalDet, was a miniature but functionally equivalent model of the MINOS Near and Far Detectors installed at CERN. It comprised 60 unmagnetized, steel planes of $1 \mathrm{~m}^{2}$ area and $2.50 \mathrm{~cm}$ width. Planes were instrumented with 24 horizontal and 24 vertical scintillator strips. The strips were read out at one end by ND and at the other by FD electronics to calibrate the differences in the two readout systems' responses. Scintillation light was carried by WLS fibers. In order to match MINOS ND and FD response, long fibers were used: $6 \mathrm{~m}$ clear fibers for one end and $3 \mathrm{~m}$ for the other. Fig. 2.22 shows a subsection of the calibration detector.

CalDet was placed in CERN PS (proton synchrotron) test beams consisting of electrons, muons, pions and protons of momenta ranging between 0.2 and $10 \mathrm{GeV} / c$ and collected data between 2001-2003. Data was collected with varying settings multiple times to achieve a run-to-run stability of better than $1 \%$ and corrected to remove systematic effects to better than $2 \%$. The resulting data was compared to Monte Carlo simulations of events using the GEANT3 [73] simulation at both the Near and Far Detectors. Fig. 2.23 shows a comparison 
of CalDet response to pions and electrons to Monte Carlo. The calibration detector is discussed in detail in [74].

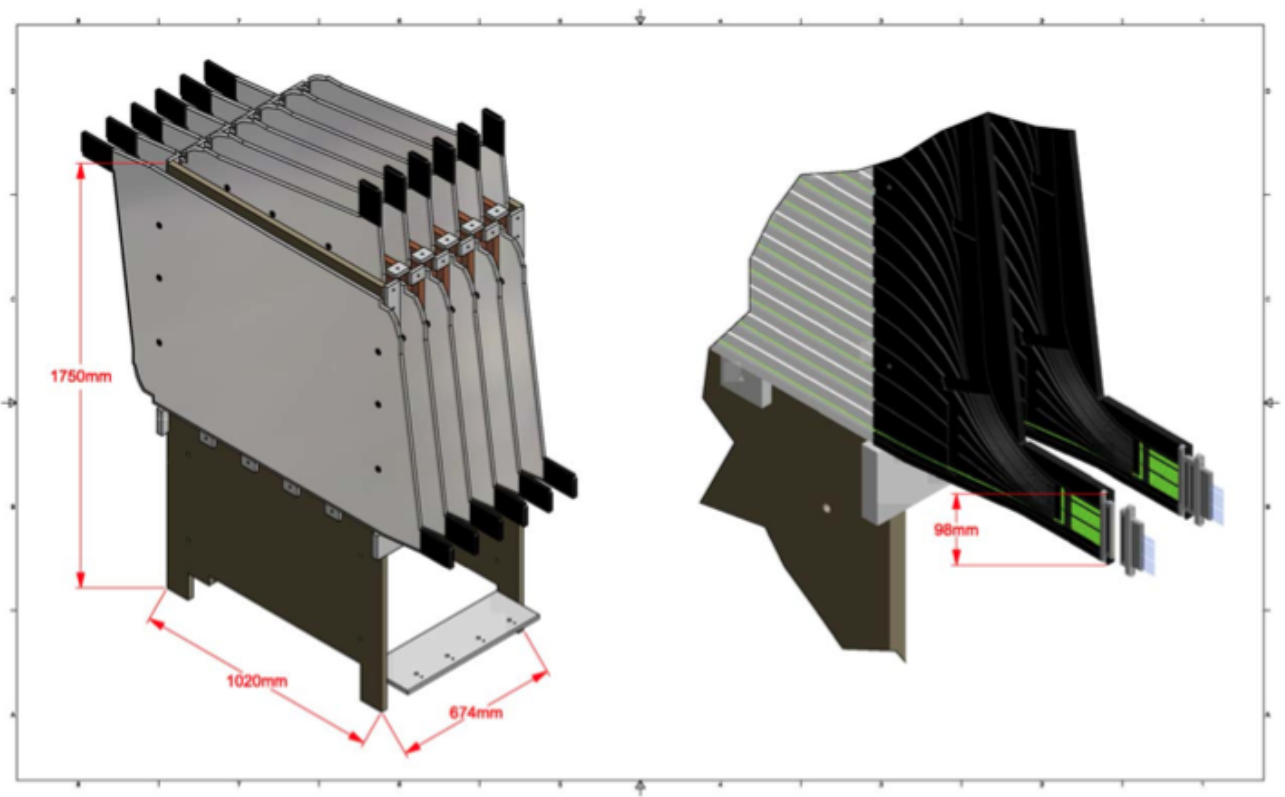

Figure 2.22: A CalDet subsection of 12 planes. The full detector was made of five such subsections. Detector planes are shown on the left and readout equipment on the right. Figure taken from [74]. 


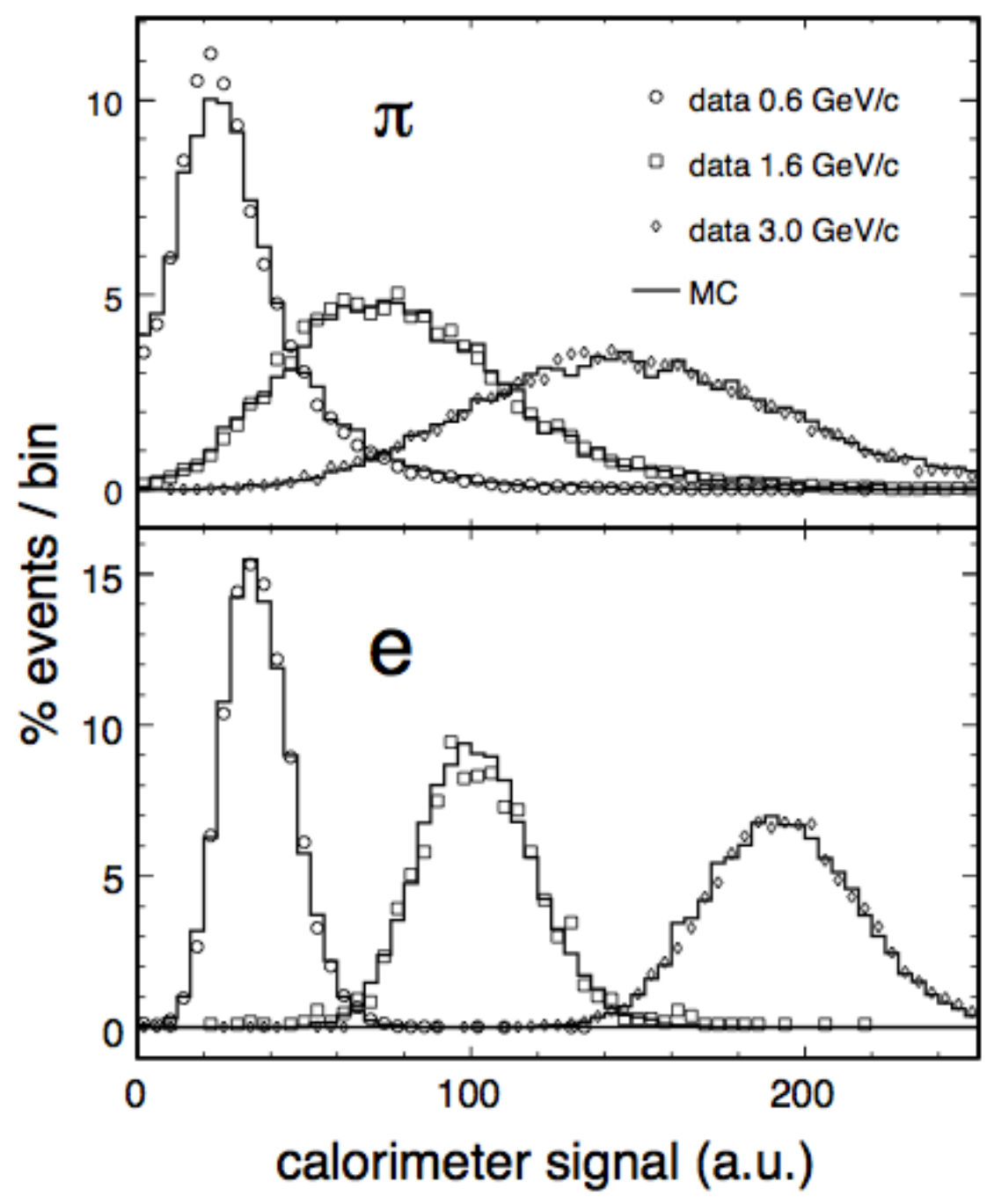

Figure 2.23: CalDet response to pions and electrons at three momenta (open symbols) compared to distributions from Monte Carlo simulations (solid lines). Figure from [71]. 


\subsection{NEAR AND FAR DETECTOR DIFFERENCES}

Although ideally the two detectors would be perfectly identical, this is not possible in reality. The event rate at the Near and Far detectors are vastly different because of the difference in their proximity to the neutrino source. The ND rate is about 10 events per spill while the FD sees a few beam neutrino interactions every day. Because of this, the FD is larger in size. Scintillator strips at the FD are longer on average than the ND. FD strips are read out at one end while ND strips are read out at both ends. Every FD plane is instrumented and read out. The ND, on the other hand, is read out at every plane in the upstream calorimeter region while only every fifth plane is instrumented in the downstream spectrometer half. The FD coil hole is at the center of the detector while at the ND it is offset. This results in small a difference in the magnetic fields. The fields are nearly identical inside the fiducial volumes. 


\subsection{EVENT RECONSTRUCTION, CALIBRATION AND MONTE CARLO SIMULATION}

The reconstruction software uses the energy deposition (pulse height), topology and timing of hits in the detector to identify neutrino interactions. The input to the reconstruction chain is a snarl, the distribution of hits in the detector that arrive in one $10 \mu$ s spill. Fig. 3.1 shows a beam spill observed in the Near Detector. A snarl is divided into individual slices based on the position and timing information of the hits. Tracks and showers are constructed from slices which are then assembled into events. Physical event properties such as position, vertex, length, angle and momentum of tracks, energy of showers are measured. An event may have more than one track or shower but one of each is defined as the primary track and shower. The event vertex is assigned as the first hit in the primary track. Section 3.1 details the steps of the reconstruction process.

Calibration, discussed in Section 3.2, aims to guarantee that the energy measurement is accurate independent of time, in each strip of each detector and across the entire energy range. It also corrects for the relative Near/Far detector differences. This is particularly important for the oscillation analyses which obtain the Far Detector prediction by extrapolating the Near Detector data (see Sec. 4.7.

Processes in particle physics are probabilistic and event rates are low, especially for neu-

trino experiments. Simulations are needed to model physical processes and the experimental apparatus. 


\subsection{RECONSTRUCTION}

\subsubsection{Slice Formation}

Due to the high intensity of the neutrino beam, one snarl in the Near Detector contains multiple neutrino interactions. Therefore, the first step in the reconstruction chain is dividing the snarl into slices, which are clusters of hits localized in space and time. Calorimeter strips with a charge deposition greater than two photoelectrons (pe), a time gap between two strips of shorter than $20 \mathrm{~ns}$ and a total time span of less than 300ns are formed into a slice. Slices must contain at least two strips. If the slice thus formed has two strips with a spatial gap of greater than $1 \mathrm{~m}$ in the longitudinal direction, the slice is split into two, provided it doesn't span the coil hole. Calorimeter strips with a charge smaller than 2 pe and spectrometer strips are added to the most suitable slice based on timing information.

In theory, Far Detector snarls are also sliced into multiple events, but the event rate at the FD is so low that this is rarely necessary in practice. In fact, there are no events in most snarls at the FD.

\subsubsection{Track Reconstruction}

Once slices are formed, the next link in the reconstruction chain is the application of a track-finding algorithm. This algorithm uses a Hough transform [76] to find track segments consisting of several hits aligned approximately linearly across several detector planes.

These segments are then added together to form a track candidate. This candidate track is fit using a multi-pass Kalman Filter [77]. The filter works by moving up and down the track estimating the state of the muon at each point along the track. It includes the effects of noise and multiple scattering in its estimate as well as the expected curvature in the magnetic field [75]. Based on this information the filter decides if a given hit should or should not be part of the track. After two passes with the filter the track momentum, or more precisely, charge-to-momentum ratio $(\mathrm{q} / \mathrm{p})$, is obtained from fitting the trajectory of the track. Other track properties are also measured at this time in addition to the momentum, such as track

beginning and end positions in two views and the uncertainty in the charge/momentum 

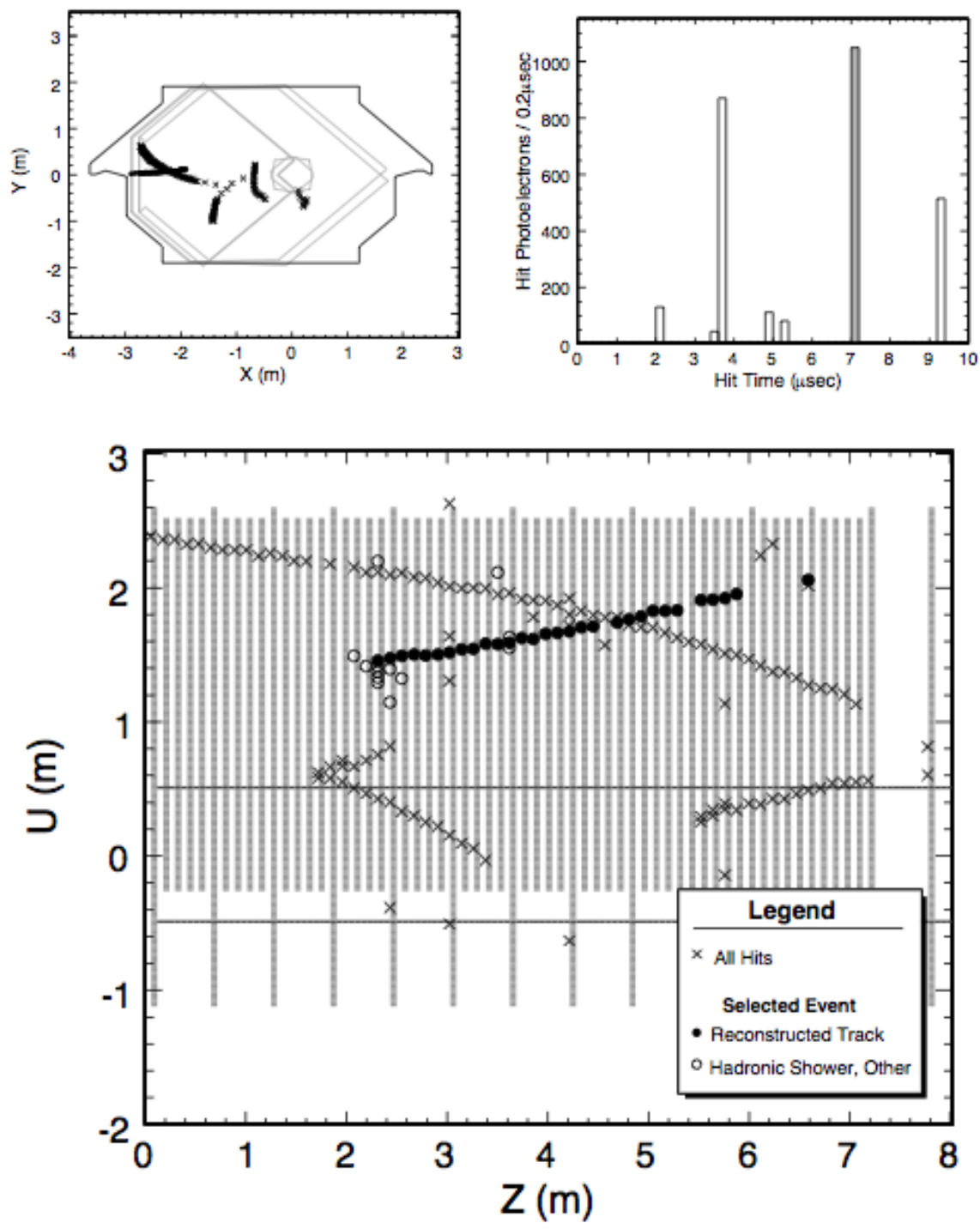

Figure 3.1: One snarl observed in the Near Detector. On the top left the beam's-eye-view of the spill where multiple events are distributed in position are shown. The top right figure shows the distribution as a function of arrival time within one spill. The bottom figure shows a reconstructed track in one detector view where gray vertical lines indicate scintillator. Figure is taken from [75].

measurement. At $3 \mathrm{GeV}$ the momentum measurement from curvature has a resolution of $11 \%$. 
If the track ends in the detector, a second, more accurate measurement of its momentum is obtained from range. This measurement has a resolution of $4.6 \%$ and is used for all tracks that do not exit the detector or end in the uninstrumented coil hole region. This task is performed by the swimmer which swims a muon backwards along the track from the track end to the beginning and sums the energy it should have lost in the steel and scintillator using the GEANT3 simulation [73].

Muons with energies between $10 \mathrm{MeV}$ and $10 \mathrm{GeV}$ lose energy through ionization which is described by the Bethe-Bloch equation and the energy loss in various media are tabulated by Groom in [78]. CalDet (see Sec. 2.7) demonstrated the accuracy of the Bethe-Bloch description, while the uncertainty in the agreement between data and simulation obtained by incorporating the Groom tabulation into GEANT3 is $2 \%$. This is taken as a systematic

uncertainty on the measurement from range. The curvature measurement for stopping tracks is found to agree with the range measurement to within $1 \%$ which was conservatively added linearly for a $3 \%$ uncertainty estimate on the curvature measurement.

\subsubsection{Cluster Formation and Shower Reconstruction}

Once the track is identified, the remaining hits localized in space and time are clustered together to construct the shower. Hits that are part of a track but have more energy than what the muon would have deposited are added to the shower after the muon part is removed. Only hits with an energy deposition of greater than two photoelectrons are considered since the low pulse height region is difficult to model. Muon energies are reconstructed topologically but the detector granularity is too coarse to distinguish individual components of a shower. There are two methods to measure the shower energy described below.

One method of measuring the shower energy is calorimetry where the energy of the shower is calculated by summing the energy deposited by all of its constituent hits (after the energy deposited in the steel is recovered). By this method, the hadronic shower energy resolution is $56 \% / \sqrt{E} \bigoplus 2 \%$ and the electromagnetic shower energy resolution is $21.4 \% / \sqrt{E} \bigoplus 4 \%$.

Another way of measuring shower energies is the $k$-nearest neighbors $(k \mathrm{NN})$ method, where shower energies are reconstructed by using the amount of deposited energy as well as 
the spatial distribution of energy deposition in the shower. The method is presented in great detail in [79] and summarized here.

For a $D$-dimensional $k \mathrm{NN}, D$ variables that are correlated with the shower energy construct a $D$-dimensional space. The distribution of Monte Carlo events in this space are known, since their true shower energies as well as the true values of the $D$ variables are known. The $k$ nearest neighbors of the query event, whose shower energy is being estimated, are found by computing its Euclidean distance to each of its neighbors and taking the $k$ closest ones. Let $\vec{y}$ be the query event we seek to identify and $\vec{x}$ the Monte Carlo event whose true parameters are known. The squared distance between the two is given by

$$
\Delta s^{2}=\sum_{i=1}^{D} \frac{\left(y_{i}-x_{i}\right)^{2}}{\sigma_{i}^{2}},
$$

where $\sigma_{i}$ is the standard deviation of the variable $i$. It is necessary to normalize the distance by this standard deviation because the $D$ input variables do not necessarily have the same units, and if un-normalized the one with the widest numerical range would dominate.

For this estimator $D=3$ variables were chosen:

- Calorimetric energy in the first two showers,

- total energy deposited within a $1 \mathrm{~m}$ radius of the track vertex,

- number of planes in the primary shower.

The number of variables, $D$, and the number of neighbors, $k$ were optimized to maximize the sensitivity to oscillations [79]. Studies with varying the number of $k$ neighbors yielded that the optimal number of neighbors with maximum sensitivity for the size of the Monte Carlo sample used was found to be $k=400$. The shower energy is estimated separately for Near and Far detector events using the corresponding Monte Carlo samples.

Ideally the reconstructed shower energy will be identical to the true energy. This is not the case in reality, and the deviation of the ratio $E_{\text {reco }} / E_{\text {true }}$ from one is energy dependent; below $5 \mathrm{GeV}$ it goes up to $15 \%$. To remedy this a 7 th degree polynomial, energy dependent correction is applied to the $k \mathrm{NN}$ shower energy estimate. The final estimate after the energy correction improves the shower energy measurement across all energies with the greatest 
improvement at low energy where the resolution is better than the calorimetric estimate by up to $25 \%$.

\subsubsection{Event Formation}

Events are formed by combining tracks and showers that are considered to be coming from the same neutrino interaction based on a set of criteria. A track is associated with a shower if the shower is within $\pm 35 \mathrm{~cm}$ of the track location in both $U$ and $V$ views. If there is no match satisfying this criteria then a number of tests are formed to determine showers associated with a track [80]. Once all associations between reconstructed objects are found, the next step is to build an event. In the most frequent and simplest case there is one track and one shower in a slice which make up an event. In cases where there are multiple tracks or showers, the track with the highest pulse height deposition (highest energy track) is selected as the primary track. Additional tracks and showers are added to the event if they meet various sets of association conditions with the existing components of the event; a logical algorithm loops through all possible associations until the event includes all objects that are believed to belong to it. If there is no track, the initial seed is a shower and its associated showers are added to the event in a similar fashion.

\subsection{CALIBRATION}

A light injection system is used to measure the linearity and time variation of PMTs and electronics response, and cosmic ray muons are used to measure variations in scintillator strip light output with time and position. No sources of known-energy particles are available to the Near and Far Detectors, but data from the calibration detector (Sec. 2.7) provides measurements to determine the relative and absolute energy scales of both detectors.

Calibration is a multi-stage process that converts the raw pulse height, $Q_{\text {raw }}$, to the fully corrected signal, $Q_{\text {corr }}$ via a multiplicative calibration constant at each stage, Eq. 3.2. The pulse height and calibration constants are functions of the scintillator strip, $s$, position, $x$, 
time, $t$ and detector, $d$.

$$
\begin{aligned}
Q_{\text {corr }}= & Q_{\text {raw }}(s, x, t, d) \times D_{\mathrm{PMT}}(d, t) \times L\left(d, s, Q_{\text {raw }}\right) \\
& \times D_{\text {scint }}(d, t) \times S(s, d, t) \times A(d, s, x) \times M(d)
\end{aligned}
$$

where $D$ is the drift correction that accounts for changes in scintillator, PMT and electronics response with temperature and time; $L$ linearizes the response of each channel; $S$ removes differences from strip to strip; $A$ corrects for attenuation depending on the position of the hit in the strip, and $M$ is the energy scale factor that corrects the pulse height to the same energy unit in both detectors.

\subsubsection{Calibration Procedure}

3.2.1.1 PMT and Electronics Drift $\boldsymbol{D}_{\mathbf{P M T}}(\boldsymbol{d}, \boldsymbol{t}) \quad$ The light injection (LI) system [81, $82]$ is designed to monitor and compensate for changes in the PMT gains and non-linearity. It uses light-emitting diodes (LEDs) to inject ultraviolet light into the wavelength shifting fibers, simulating scintillator light. 20 LEDs are housed in a pulser box and illuminate multiple individual fibers. The intensity of injected light is monitored by PIN diodes while simultaneously read out by PMTs. The LI system pulses periodically at the end of every strip: 300 times per hour at the FD and 1000 times per hour at the ND. This is designed to average 50 photoelectrons per pulse per PMT pixel in both detectors. Changes in LED intensity can lead to variations of up to a factor of two in this light level; such changes are monitored and corrected.

This LI data is collected once a month at each detector. PMT gains are measured using photon statistics [81]. Gains measured by the LI system provide corrections to the PMT gain and electronics response. Short term variations that are usually due to temperature variations can be eliminated by good environmental control, while longer term variations due to aging and changes in hardware amount to $\sim 4 \% /$ year. 
3.2.1.2 Linearity $L\left(d, s, Q_{\text {raw }}\right) \quad$ PMT and electronics responses are not linear for light levels greater than approximately 100 photoelectrons. The LI system is utilized to correct for this nonlinearity by injecting a known intensity of light into scintillator strips, ranging from a few to hundreds of photoelectrons, and measuring the electronics response as a function of true illumination. In the Near Detector, the charge integration and ADC electronics are also calibrated for linearity by directly injecting charge into the front-end electronics

3.2.1.3 Scintillator Drift Correction $D_{\text {scint }}(d, t)$ The scintillator response changes in time as the material ages. Aging reduces the light level by about $2 \%$ annually and environmental variations, mainly temperature, result in short-term changes. The response of the detectors is measured by monitoring the median response of a detector plane to through-going cosmic muons. Although the spectra of cosmic muons are different between detectors, due to differences in depth, zenith angle and latitude, the energy spectra and rate of cosmic muons going through one detector is time independent. It is therefore possible to calibrate for this drift by comparing the median detector response at time $t$ to the response at time $t_{0}$, the beginning of the experiment:

$$
D_{\text {scint }}(d, t)=\frac{\text { Median response }\left(d, t_{0}\right)}{\text { Median response }(d, t)}
$$

3.2.1.4 Strip-to-strip Calibration $\boldsymbol{S}(s, \boldsymbol{d}, \boldsymbol{t}) \quad$ The strip-to-strip non-uniformity correction accounts for several detector effects including scintillator light yield, WLS collection efficiency, differences in attenuation optical fibers, PMT quantum efficiency and PMT gain. The correction is obtained by using cosmic ray muons in the minimum ionizing energy range (a few $\mathrm{GeV}$ ), since these muons' energy deposition is relatively independent of their momenta. An individual strip's response is characterized by constructing the muon spectrum for that strip from cosmic ray muon data accumulated over a period of time. The calibration constant is then calculated monthly as the strip response normalized by the mean response of all strips:

$$
S(s, d, t)=\frac{\text { Mean response of whole detector }(d, t)}{\text { Response of strip }(s, d, t)}
$$


3.2.1.5 Attenuation $\boldsymbol{A}(\boldsymbol{d}, \boldsymbol{s}, \boldsymbol{x})$ The response of one strip can show variations between different transverse positions on the strip mainly because of WLS attenuation. The calibration constant is given by,

$$
A(d, s, x)=A_{1}^{d, s} e^{-x / L_{1}^{d, s}}+A_{2}^{d, s} e^{-x / L_{2}^{d, s}}
$$

where $x$ is the transverse position along the strip and $L_{1}^{d, s}$ and $L_{2}^{d, s}$ are the attenuation lengths. This correction was calculated by using a module mapper. The module mapper measured the response of each scintillator module to ionizing radiation using a ${ }^{137} \mathrm{Cs}$ source. To obtain the calibration constant, i.e., the values of $A_{1}, A_{2}, L_{1}$ and $L_{2}$, a fit is performed for each strip and resulting parameters are used to correct the data [71]. The attenuation correction was later cross checked by using through-going muons and was found to be consistent to within $4 \%[68]$.

3.2.1.6 Inter-detector Calibration $\boldsymbol{M}(\boldsymbol{d}) \quad$ In addition to correcting each of the three detectors' responses to be spatially and temporally consistent within one detector, the calorimetric responses of the Near and Far Detectors and CalDet are calibrated to normalize the energy scales of the three detectors. This calibration is performed by using the stopping cosmic ray muons sample. Stopping cosmic muons make up a small fraction of the total cosmic sample $(\sim 0.3 \%$ at the FD) but there are enough cosmic rays that stopped muons are a sufficient dataset for this purpose. Stopping muons are used because their energy depositions are well understood, especially between $0.5 \mathrm{GeV}$ and $1.1 \mathrm{GeV}$ where their $\frac{d E}{d x}$ is nearly constant. Because of this, the track window technique is used, where the end $0-0.5$ GeV portion of the track is discarded. The detectors' energy scales are normalized to within the target of $2 \%$ using this method [71]. To be able to compare the response of the ND, FD and CalDet a unit of muon energy equivalent (MEU) is defined as the detector response to a perpendicular minimum ionizing particle in the energy range $0.5-1.1 \mathrm{GeV}$ traversing one scintillator plane. To determine the constant relating the measured signal to actual energy loss, CalDet data that lie in the track window were fit to the Bethe-Bloch equation, which governs the energy loss of a muon by ionization. 


\subsubsection{Absolute Energy Scale}

The absolute track and shower energies were determined using data collected by CalDet while it was exposed to various test beams of known composition and energy at CERN. CalDet collected data with pions, protons, electrons and muons of both positive and negative charge signs in the energy range $200 \mathrm{MeV}$ to $10 \mathrm{GeV}$ while other detectors in the beamline were utilized to identify the particles. The range of stopping muons (of momenta smaller than 2.2 $\mathrm{GeV} / c$ ) was modeled to better than $3 \%$ accuracy with GEANT3, while the detector response to electrons was simulated with an accuracy better than $2 \%$. The pion and proton induced showers were reproduced to better than $6 \%$ accuracy with the GCALOR [83] simulation.

\subsection{MONTE CARLO SIMULATION}

Monte Carlo models interactions by repeatedly simulating each neutrino interaction and final state particle, which has a probability density function (PDF), a large number of times so that the available phase space is sufficiently sampled. To construct a reliable simulation, each component of the physical processes and the experiment need to be modeled. For MINOS, these can be broken down into two steps: the simulation of the neutrino flux, and the simulation of interactions of these neutrinos with detectors.

The flux simulation relies on the FLUGG [68] package, which combines the FLUKA [84, 85] software for particle interactions and propagation with the GEANT4 [86, 87] geometry package. The flux simulation models hadron production at the target, focusing by horns, and subsequent decays which produce neutrinos in the decay pipe. The FLUGG package is able to model the beamline with a detailed description of the target position (including a $1 \mathrm{~cm}$ shift for part of the datataking period) and the effects of helium in the decay pipe for run periods after November 2007. The flux is simulated separately for each run period to account for these effects accurately. The results of the simulation are "flux files" that contain details of hadrons, muons and the daughter neutrinos from their decays.

For the second step of the simulation, the NEUGEN3 [88] neutrino interaction generator 
software simulates neutrino interactions by using the above flux files as input. NEUGEN simulates both the neutrino interaction in the detector and the reinteraction of final state hadrons with nuclear matter. Interactions in the surrounding area and rock are simulated and overlaid with the detector interactions. The effect of multiple interactions in the same spill are also taken into account by overlaying individual simulated events [89] at this stage.

Once neutrino interactions are generated, GMINOS [90] simulates the propagation of particles through the detector. The modeling of the propagation is energy dependent and the simulation includes a detailed model of the detector geometry from GEANT3. Hadronic showers are modeled by the GCALOR [83] software chosen for its good agreement with data from the calibration detector (see Sec. 2.7). This part of the simulation outputs energy deposition in the detector.

This resulting energy deposition is converted into light and taken through the WLS fiber to the PMT cathode where it is converted to photoelectrons by the MINOS package PhotonTransport. Another MINOS package, DetSim, simulates the next steps: photomultiplier tubes and readout electronics. Detailed information about PMTs and electronics' behavior is included in the simulation, such as cross-talk, non-linearity, and noise.

To best represent real interactions, the simulated energy deposition signal is uncalibrated by applying the calibrations described in section 3.2 in reverse before conversion to light. In order to determine which calibration constants to use, the simulated data is assigned a random date from the pool of dates when actual data was collected so that data and Monte Carlo have the same distribution of calibration constants. This fake date is recorded and when Monte Carlo is calibrated the appropriate constants are used.

The effect of neutrino oscillations in Monte Carlo is taken into account during analysis by reweighting the events and by using special "tau files." These tau files contain events in a scenario where the oscillation probability $P\left(\nu_{\mu} \rightarrow \nu_{\tau}\right)=1$, whereas the standard Monte Carlo is unoscillated with $P\left(\nu_{\mu} \rightarrow \nu_{\mu}\right)=1$. Then the spectra in any oscillation scenario can be constructed by an appropriate linear combination of the standard Monte Carlo and these tau files. 


\subsection{ANTINEUTRINO OSCILLATION ANALYSIS}

To measure antineutrino oscillation parameters the Far Detector observed event rate as a function of energy is compared with prediction. We fit for the mixing angle and mass splitting between the second and third mass states in the two-flavor oscillation model explained in Section 1.3.

The data used to measure antineutrino oscillations was collected between 2009 and 2011 during two run periods in reversed horn current (RHC) mode. MINOS quantifies the amount of data in units of protons-on-target (POT): the number of protons striking the graphite target. A total of $2.95 \times 10^{20} \mathrm{POT}$ antineutrino-mode data was analyzed to measure antineutrino oscillations. This chapter details the steps of the analysis and presents the results.

\subsection{EVENT TOPOLOGIES}

There are multiple event topologies in the MINOS detectors. Charged-current $\nu_{\mu}$ and $\bar{\nu}_{\mu}$ interactions leave a $\mu^{-}$and $\mu^{+}$in the detector respectively. The muon energy deposition pattern produces a long track that curve in the magnetic field. In neutrino-mode running, the toroidal field curves a $\mu^{-}$towards the coil hole and $\mu^{+}$away. Events curving towards the coil hole are more likely to be contained allowing their energy to be measured from range which is more accurate than the measurement by curvature. Exiting events' momenta are

measured from curvature. In antineutrino-mode the detector field is reversed and $\mu^{+}$tracks are focused.

$\mathrm{NC}$ and $\mathrm{CC} \nu_{e}$ interactions produce events without tracks or with very short tracks. They are characterized by showers. NC showers are more diffuse while $\mathrm{CC} \nu_{e}$ showers have 
a tighter core and a smaller radius. $\mathrm{NC}$ and $\mathrm{CC} \nu_{e}$ interactions are studied separately but for the analyses presented in this dissertation both are background; they are not treated separately. Since there are many more NC events than $\mathrm{CC} \nu_{e}$, the latter is folded in with the former. Fig. 4.1 shows the signatures of CC and NC events in the MINOS detectors.
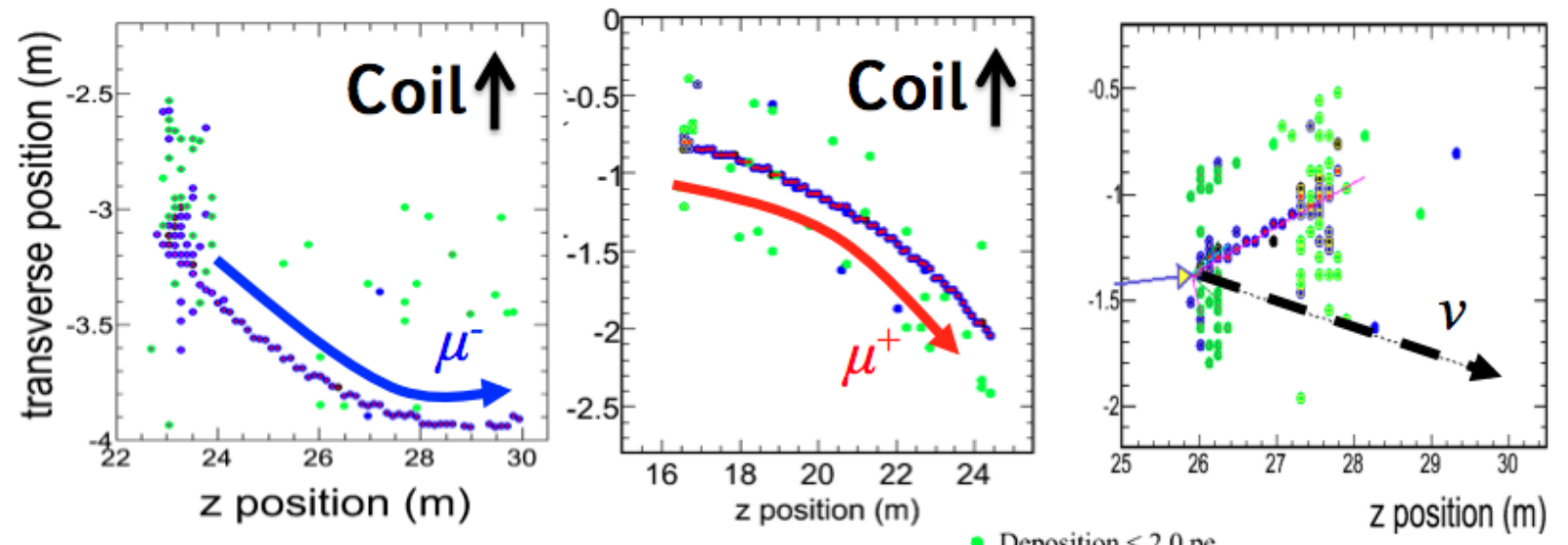

Simulated Events

- Deposition $<2.0 \mathrm{pe}$

- $2.0<$ Deposition $<20.0$ pe

- Deposition $>20.0$ pe

Figure 4.1: Event displays showing simulations of a $\mathrm{CC} \nu_{\mu}$ (left), a $\mathrm{CC} \bar{\nu}_{\mu}$ (center) and NC (right) interaction. Green hits denote energy deposited less than 2 photoelectrons, blue shows 2 to 20 pe and black greater than 20 pe. The red dots indicate hits of the reconstructed event. CC $\nu_{\mu}$ and $\bar{\nu}_{\mu}$ interactions produce a $\mu^{-}$and a $\mu^{+}$respectively, which can be identified from the opposite directions in which they curve in the magnetic field. NC events usually have no muon tracks. 


\subsection{EVENT SELECTION}

Near and Far detector data samples are obtained by requiring that events meet a series of selection criteria. The pre-selection common to both detectors ensures data quality. Each detector has its own fiducial volume requirements. The analysis selection is designed to separate positively and negatively charged final state muons and separates charged current $\bar{\nu}_{\mu}$ interactions from neutral currents.

\subsubsection{Pre-selection}

Before analysis-specific event selection, all events need to meet a set of data quality criteria. It is required that NuMI is operating in the low-energy mode (see Fig. 2.7) for which the target sits inside the first horn, the separation between the two horns is $10.0 \mathrm{~m}$ and the horn current is set to $185 \mathrm{kA}$. The direction of the horn current in antineutrino mode is such that it focuses negatively charged mesons and deflects positively charged ones. Both detector coils need to be on so that the detectors are magnetized. In antineutrino-mode the coil current is 'reversed' to focus positively charged particles towards the coil hole. For an event to be included in the data sample it must arrive within a beam spill so that atmospheric and cosmic background is reduced. Events are required to be reconstructed with at least one muon track, since all events relevant to this analysis are charged-current interactions with a muon in the final state. Track vertices are required to lie within the respective fiducial volumes of the detectors; this requirement aims to guarantee that all events have their hadronic shower fully contained inside the detector.

\subsubsection{Far Detector Fiducial Volume}

The event rate at the Far Detector is low (a few neutrino interactions per day) and it is essential to record and keep as many events as possible to maximize the statistical power of the analysis. The FD fiducial volume is therefore chosen to be as large as possible. FD events

must be inside a radius range of $40 \mathrm{~cm}<R<\sqrt{14} \mathrm{~m}$. The inner limit excludes events too close to the un-instrumented magnetic coil region where reconstruction is not well modeled. 
The outer limit guarantees that the edge of the fiducial volume is never closer than $35 \mathrm{~cm}$ to the edge of the detector (the detector is octagonal while the fiducial volume is cylindrical). In addition, the FD fiducial volume excludes the first three planes of either supermodule, removing events that originate outside the detector (in the surrounding rock) and enter through the face. The last ten planes of the first and last 22 of the second supermodules are also excluded to remove events whose hadronic showers would exit out of the back of the detector. Fig. 4.2 shows the Far Detector fiducial volume graphically.

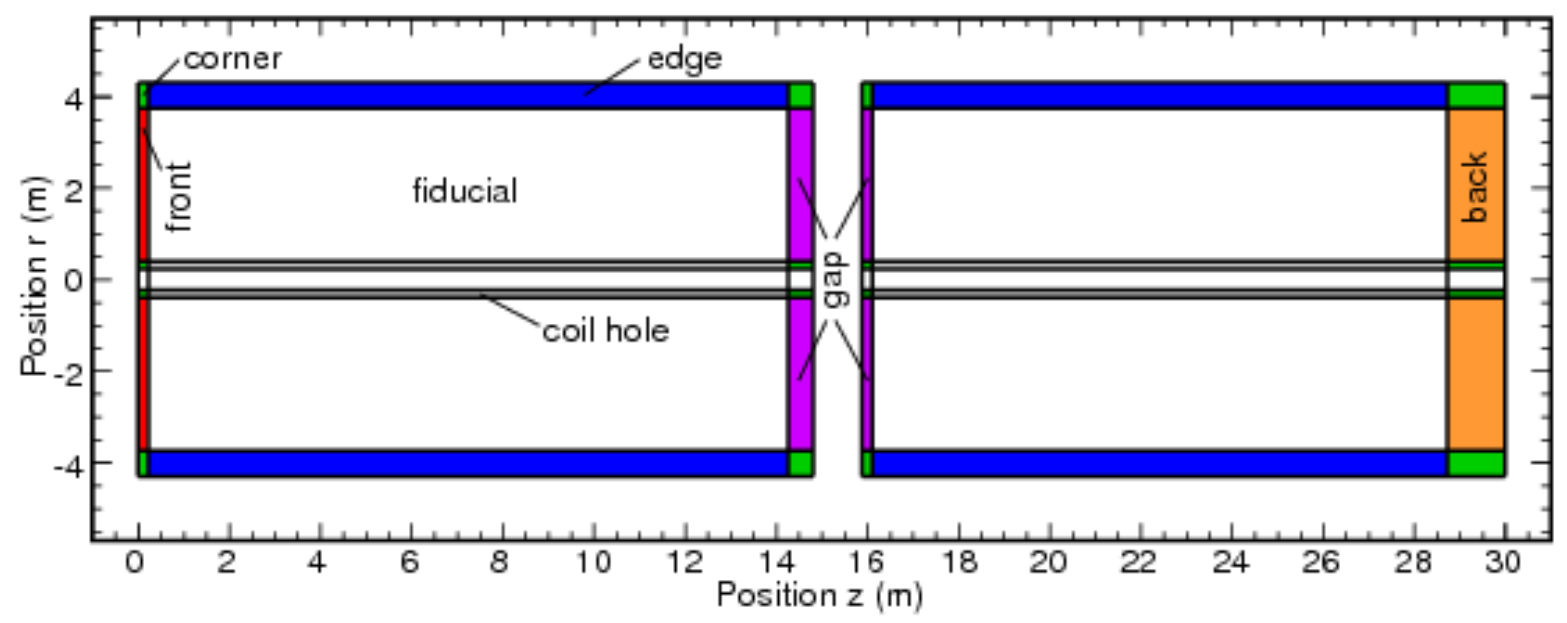

Figure 4.2: The Far Detector fiducial volume is as large as possible; excluded areas are shown in color.

\subsubsection{Near Detector Fiducial Volume}

At the Near Detector the event rate is high and the fiducial volume is smaller allowing a purer sample. ND event vertices are required to lie radially within $80 \mathrm{~cm}$ of the beam center, and longitudinally between planes 14 and 68 . 


\subsubsection{Separation of charged-current and neutral-current events}

Fig. 4.1 shows the signatures of the two main kinds of muon neutrino interactions in the MINOS detectors: charged-current (CC) and neutral-current (NC). CC antineutrino interactions (middle panel) are the signal we need to extract for this analysis and the first step is to distinguish charged and neutral current interactions.

In order to do this an algorithm called $k$-Nearest Neighbors $(k N N)$ is employed. This is a multivariate method in which an N-dimensional space of track variables is constructed. A query event is identified as signal-like (CC) or background-like (NC) by its $k$ neighbors' characteristics. The distance in $k \mathrm{NN}$ space is determined by

$$
D=\left(\sum_{i=1}^{d}\left|X_{i}^{M C}-X_{i}^{Q}\right|^{2}\right)^{\frac{1}{2}}
$$

where $\vec{X}$ is a vector in $k \mathrm{NN}$ multivariate space with $M C$ denoting the Monte Carlo events of known type (charged or neutral current) and $Q$ denoting the query event. The probability that the query events is a signal event is then given by the particle identification parameter $(k \mathrm{NN} \mathrm{PID})$ is then given by

$$
k \mathrm{NN}_{\mathrm{ID}}=\frac{k_{S}}{k_{S}+k_{B}}
$$

where $S$ and $B$ denote signal and background respectively. In the cartoon in Fig. 4.3, red dots denote signal and blue dots background. In this case $k=12, k_{S}=8, k_{B}=4$ which gives the query event marked by the star a $k \mathrm{NN}_{\mathrm{ID}}$ of $\frac{2}{3}$.

In general the $\mathrm{N}$ variables which make up the $k \mathrm{NN}$ space have different units. The variables need to be normalized in order to avoid an arbitrary bias resulting in some of the variables having larger weight in the overall discrimination power (i.e. an ellipse containing a different set of events instead of the circle in the cartoon in Fig. 4.3). The normalization scale factor for each variable is determined to be the width of its distribution.

The $k \mathrm{NN}$ space in this analysis is constructed by four variables that can distinguish between charged and neutral current events (shown in Fig. 4.5) and uses $k=80$ neighbors. The first two variables quantify the topology of the track and the other two describe the 


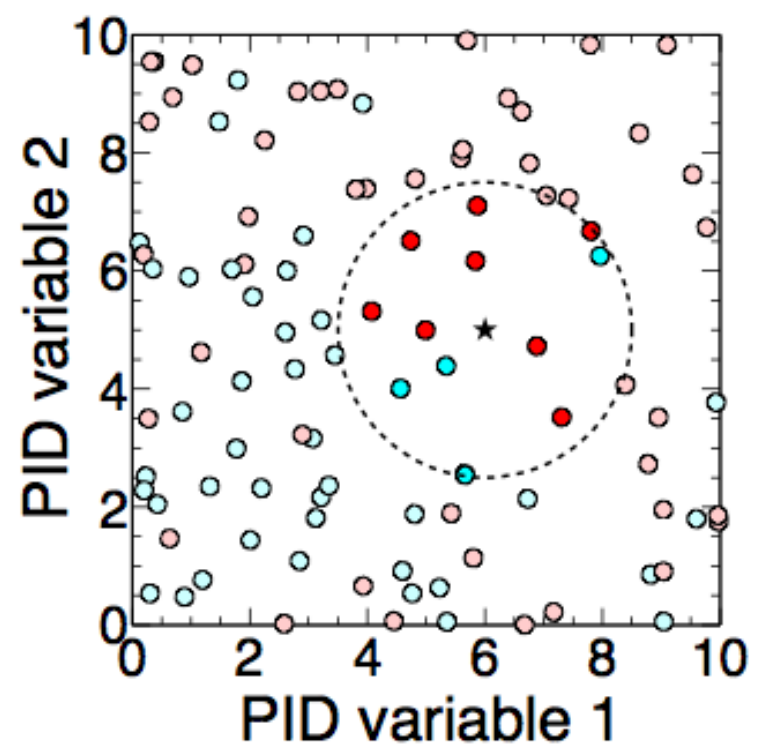

Figure 4.3: A cartoon demonstrating the use of the $k \mathrm{NN}$ particle identification in twodimensional $k \mathrm{NN}$ space. 12 nearest neighbors lie in the dashed circle, red representing signal and blue representing background. This gives the event a $k$ NN PID value of $\frac{2}{3}$.

energy deposition along the track. The value of $k$ was optimized such that the $k$ neighbors represent the local behavior of the probability density functions with little fluctuation in the classification outcome. The development and use of the $k \mathrm{NN}$ algorithm is discussed in great detail in [91].

- Number of scintillator planes in track. The number of planes that a muon penetrates is a direct measure of the length of the track. Muons can travel a long distance through matter unlike hadrons which shower, so long tracks are easily identified as muons.

- Transverse track profile. A scintillator plane is made of closely packed scintillator planes (see Fig. 2.8). A typical muon track deposits energy in one scintillator strip per plane, whereas a hadronic shower has a wider transverse profile that spreads out to a few planes. The transverse track profile parameter is computed by taking the ratio of the number of track hits to the total number of hits within a small window of space and time around the track, away from the vertex. 
- Mean pulse height of track hits. This variable measures the average energy loss along the track $(d E / d x)$ away from the vertex. The amount of energy deposited is smaller for muons which are usually minimum ionizing compared to hadronic showers that are typically more energetic.

- Signal fluctuation. The signal fluctuation is quantified by taking the ratio of the mean energies of lowest pulse height hits to the mean of the highest pulse height hits in a track. The fluctuation along the track tends to be smaller for muons whose energy loss is more or less uniform. Hadronic showers have large fluctuations in deposited energy.

The distribution of the resulting $\mathrm{CC} / \mathrm{NC}$ discriminator is shown in Fig. 4.6. The optimal position for selecting muon tracks for the antineutrino oscillation analysis was found as $k \mathrm{NN}_{\text {ID }}>0.3$. Two independent selector studies were performed to optimize the cut position, one optimizing log-likelihood and the other maximizing selection efficiency $\times$ sample purity [92]. Fig. 4.4 shows the outcome of the two methods; one finds $k \mathrm{NN}_{\mathrm{ID}}>0.22$ and the other $k \mathrm{NN}_{\mathrm{ID}}>0.39$ as the ideal cut position. As the figure indicates the distribution is flat between these two values so the middle was chosen. The exact value between 0.2 and 0.4 have negligible effect on the results.
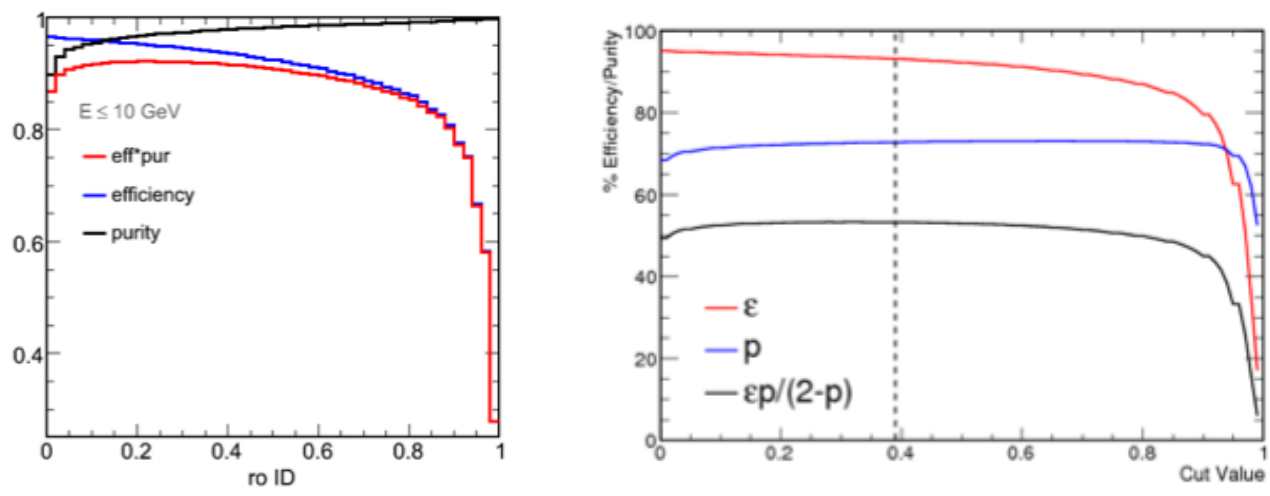

Figure 4.4: Selection efficiency $\times$ sample purity distributions of two selectors using the $k \mathrm{NN}$ $\mathrm{CC} / \mathrm{NC}$ separator. 

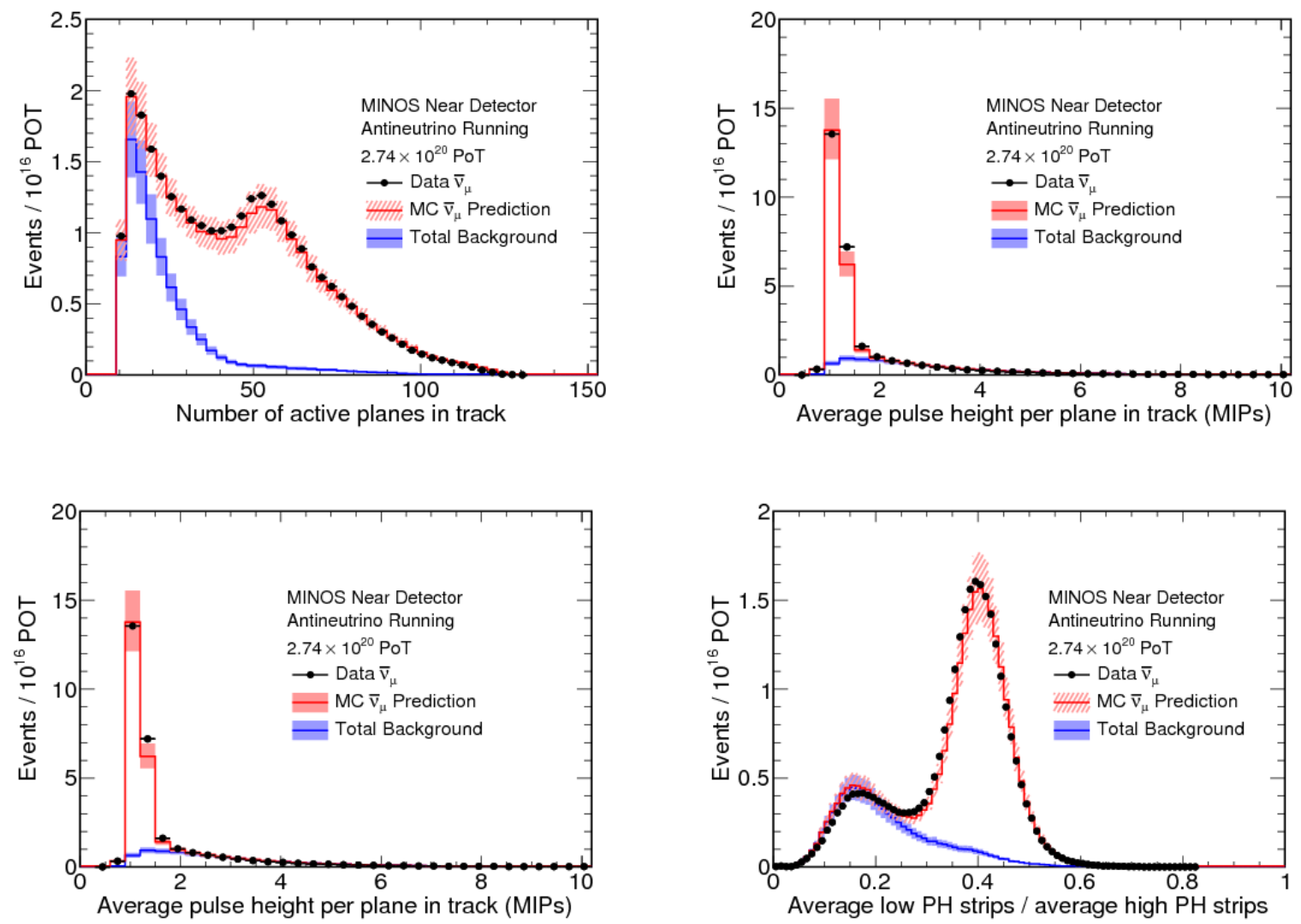

Figure 4.5: Distributions of the four $k \mathrm{NN}$ input variables before $\mathrm{CC} / \mathrm{NC}$ selection is applied. The red histogram represents charged current Monte Carlo events, blue shows neutral currents, and black dots show data. The distributions of $\mathrm{CC}$ and $\mathrm{NC}$ events are significantly different in each variable making it possible to separate the two. 


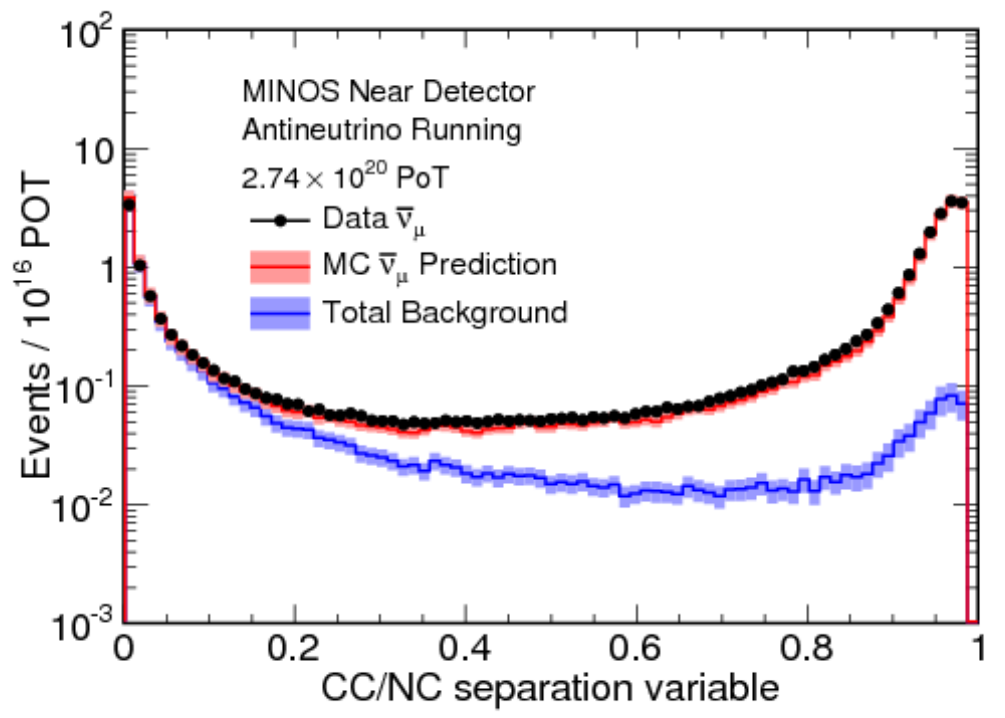

Figure 4.6: $\mathrm{CC} / \mathrm{NC}$ separation parameter of events passing all other selection cuts. The red histogram represents the Monte Carlo expectation with systematic uncertainties, the blue histogram represents the total (charged and neutral current) background with systematic uncertainties. Black points represent data. The cut removes events with $k \mathrm{NN}_{\mathrm{ID}}>0.3$. 


\subsection{CHARGE SIGN SELECTION}

The second background to remove is the wrong-sign background from the selected chargedcurrent sample. The radius of curvature of the track is proportional to its momentum; the direction depends on the charge sign. From this information the quantity charge/momentum $(q / p)$ is computed and is used to distinguish positively and negatively charged muons. The distribution of ND charged-current events before charge selection is applied is shown in Fig. 4.7. In order to select antineutrinos, events with $q / p>0$ are kept. There is a few percent neutrino contamination from $\mu^{-}$mis-identified as $\mu^{+}$(note that the blue histogram has some non-zero bins in the $q / p>0$ region in 4.7$)$.

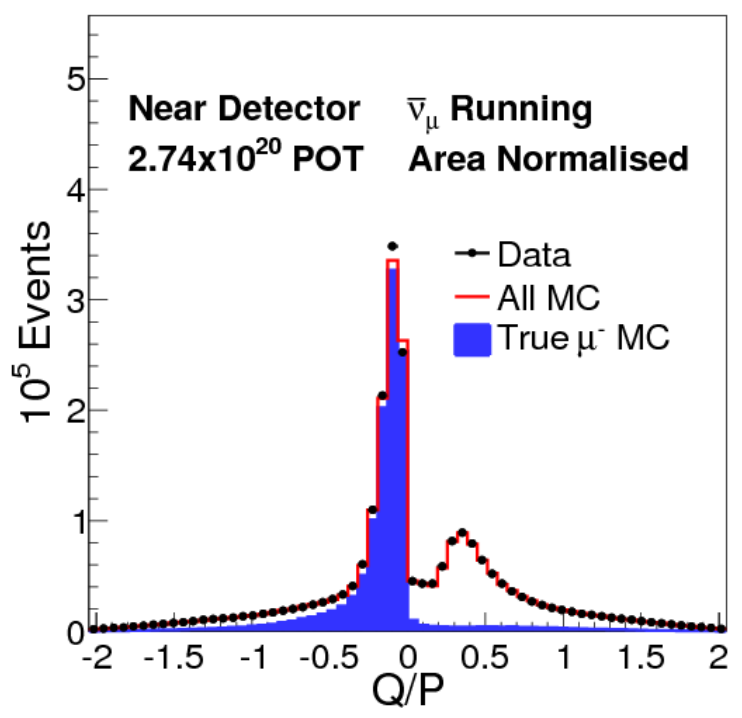

Figure 4.7: The distribution of charge over momentum for ND events before charge selection. The red curve shows all MC events, blue region shows true $\mu^{-}$tracks and black dots denote data. There are some true $\mu^{-}$misidentified to carry positive charge. 


\subsection{NEAR DETECTOR TRACK END CUT}

One additional cut is applied to the Near Detector sample to remove poorly modeled events that pass through or end near the coil hole. The track fitter frequently fails or misreconstructs tracks in this region. A track end cut is applied to exclude events traveling into or through the coil hole by rejecting tracks that end to the left of the coil. Fig. 4.8 shows the distribution of events which fail the track fitter algorithm overlaid with the fiducial volume region. This coil hole cut removes the majority of events that failed the track fitter. It has been verified that this geometric exclusion does not cause a biased selection in kinematic variables, nor does it alter the Far Detector predicted spectra significantly. Fig. 4.9 shows the ND reconstructed energy distribution before and after the track end cut (in the legend 2010 selection indicates before, 2011 after).

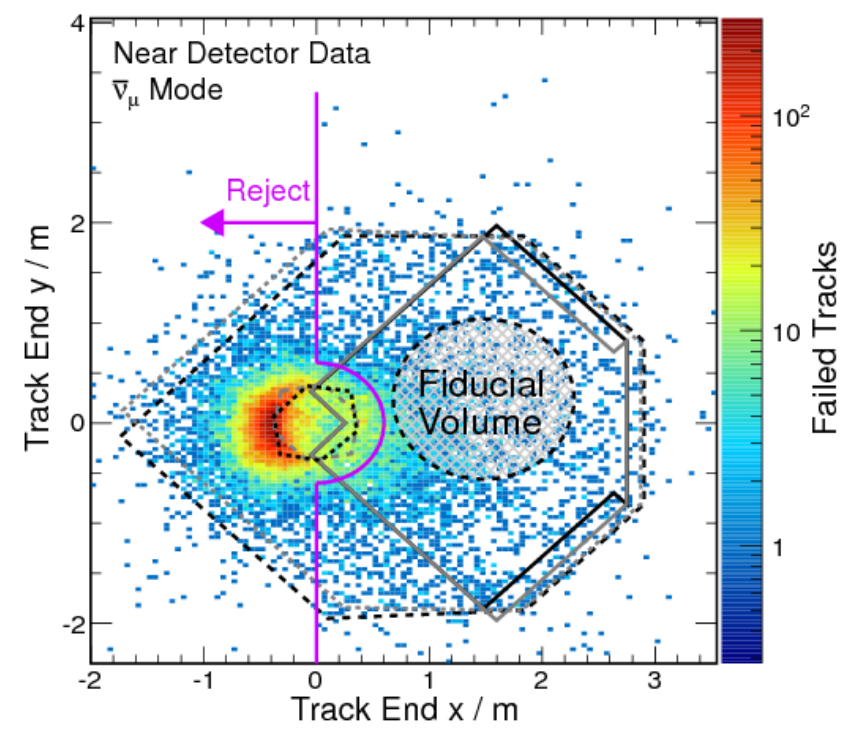

Figure 4.8: End positions of ND events that fail the track fitter. The dashed hexagon shows the coil hole region. Events to the left of the pink line are rejected removing the high concentration region (colored red) of failed tracks. 

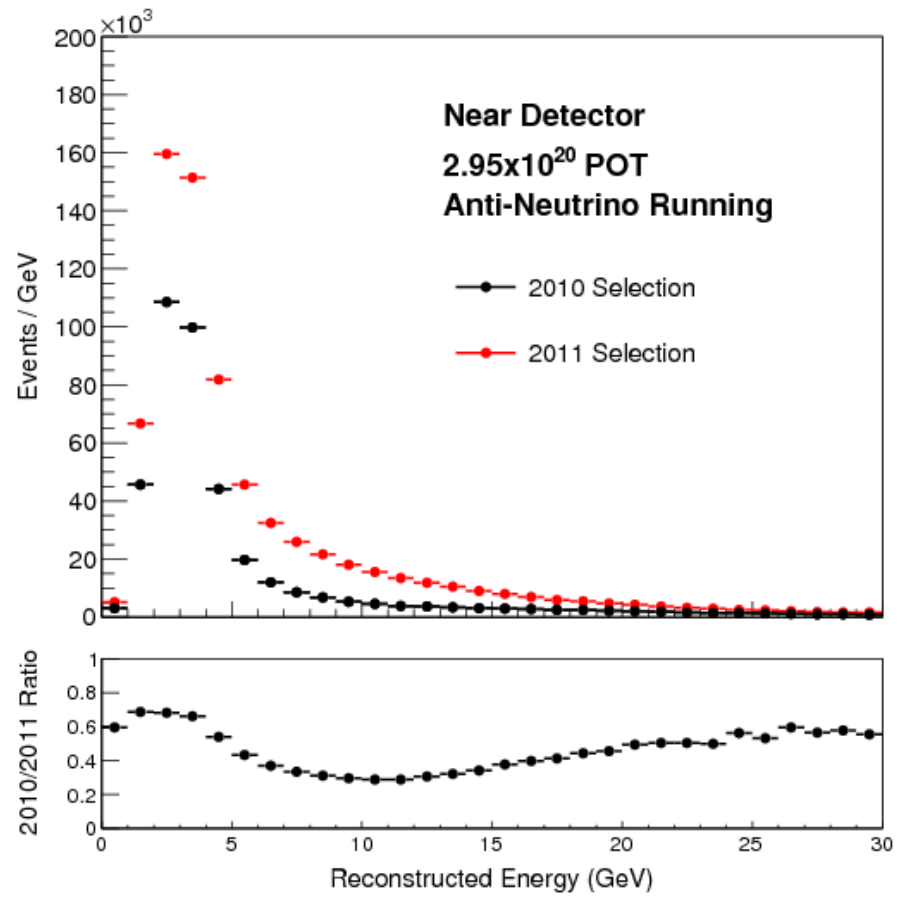

Figure 4.9: The reconstructed antineutrino energy distribution at the Near Detector with (black) and without (red) the track end cut. The bottom plot shows the ratio of the spectrum with the cut to the one without.

\subsection{BEAM TUNING}

The accuracy of the simulation of neutrino interactions in the detector depends on the accuracy of the simulation of the flux of neutrinos going through the detector, which in turn depends on the knowledge of production of pions and kaons in the target and of the focusing optics. Uncertainties in the hadron production model generate significant uncertainties in the overall neutrino flux prediction.

The built in flexibility of NuMI allowed data collection in multiple beam configurations (see Fig. 2.7). By simulating the flux in these various beam configurations and performing a multi-variable fit, the flux prediction was tuned to achieve good agreement with the observed Near Detector data. The fit constrains the $\pi^{+} / \pi^{-}$ratio by using both simulated results from 
FLUKA05 and experimental results from the NA49 experiment [93]. It is a simultaneous fit across all beam configurations and separate runs. The best fit is used to determine a weight for the pion (and kaon) which is propagated to the Near and Far detectors.

The hadron production is parametrized following the BMPT parametrization, [94],

$$
\frac{d^{2} N}{d x_{F} d p_{T}}=\left[A+B p_{T}\right] \cdot \exp \left(-C^{3 / 2} p_{T}\right)
$$

where $p_{T}$ is the transverse momentum of the meson and $x_{F}=p_{Z} / p_{\text {proton }}$ is the ratio of its longitudinal momentum to the momentum of the proton. Parameters $A\left(x_{F}\right), B\left(x_{F}\right), C\left(x_{F}\right)$, which are functions of $x_{F}$, are obtained by fitting with functions of $x_{F}$. A, $B, C$ are redefined for $\pi^{+}$as,

$$
\begin{aligned}
& A^{\prime}\left(x_{F}\right)=\left(\operatorname{par}[0]+\operatorname{par}[1] \cdot x_{F}\right) \cdot A\left(x_{F}\right) \\
& B^{\prime}\left(x_{F}\right)=\left(\operatorname{par}[2]+\operatorname{par}[3] \cdot x_{F}\right) \cdot B\left(x_{F}\right) \\
& C^{\prime}\left(x_{F}\right)=\left(\operatorname{par}[4]+\operatorname{par}[5] \cdot x_{F}\right) \cdot C\left(x_{F}\right)
\end{aligned}
$$

and similarly for $K^{+}$with parameters par[6] through par[11]. The weight of a meson is calculated as

$$
W\left(\pi^{+} / K^{+}, p_{T}, p_{Z}\right)=\frac{\left[A^{\prime}+B^{\prime} p_{T}\right] \cdot \exp \left(-C^{\prime 3 / 2} p_{T}\right)}{\left[A+B p_{T}\right] \cdot \exp \left(-C^{3 / 2} p_{T}\right)}
$$

Two additional parameters are needed to define the conversion between $\pi^{+}$and $\pi^{-}$ weights; two more to do the same for $K^{+}$to $K^{-}$. This brings the total number of hadron production fit parameters to sixteen. There are a further eight parameters in the fit to account for uncertainties in the flux measured by the Near Detector such as focusing, detector effects and target degradation. The effect of the fit on can be seen in Fig. 4.10 where the improvement of the agreement between data and Monte Carlo is apparent. The tuning was designed to be used with neutrino-mode beam configurations, and adapted to provide appropriate weights for antineutrino-mode Monte Carlo Fig. 4.11 shows comparisons of ND data to tuned and untuned MC in and antineutrino modes. 


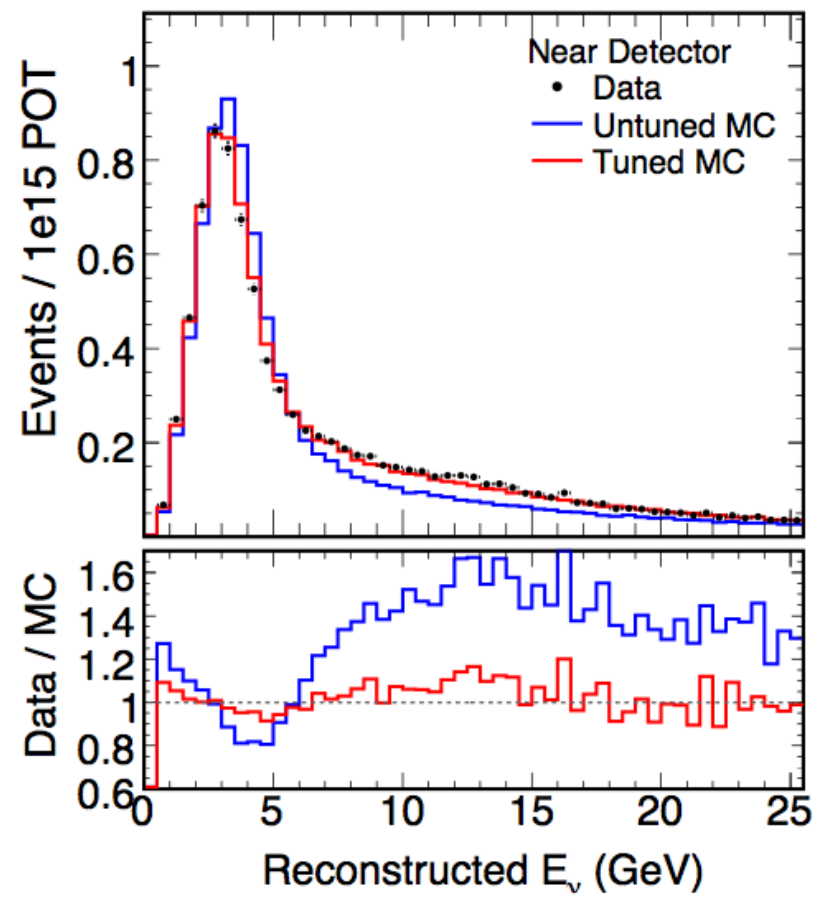

Figure 4.10: Data (black dots), untuned (blue) and tuned (red) Monte Carlo comparison of the Near Detector neutrino energy spectrum. The tuning significantly improves the agreement between data and simulation. 

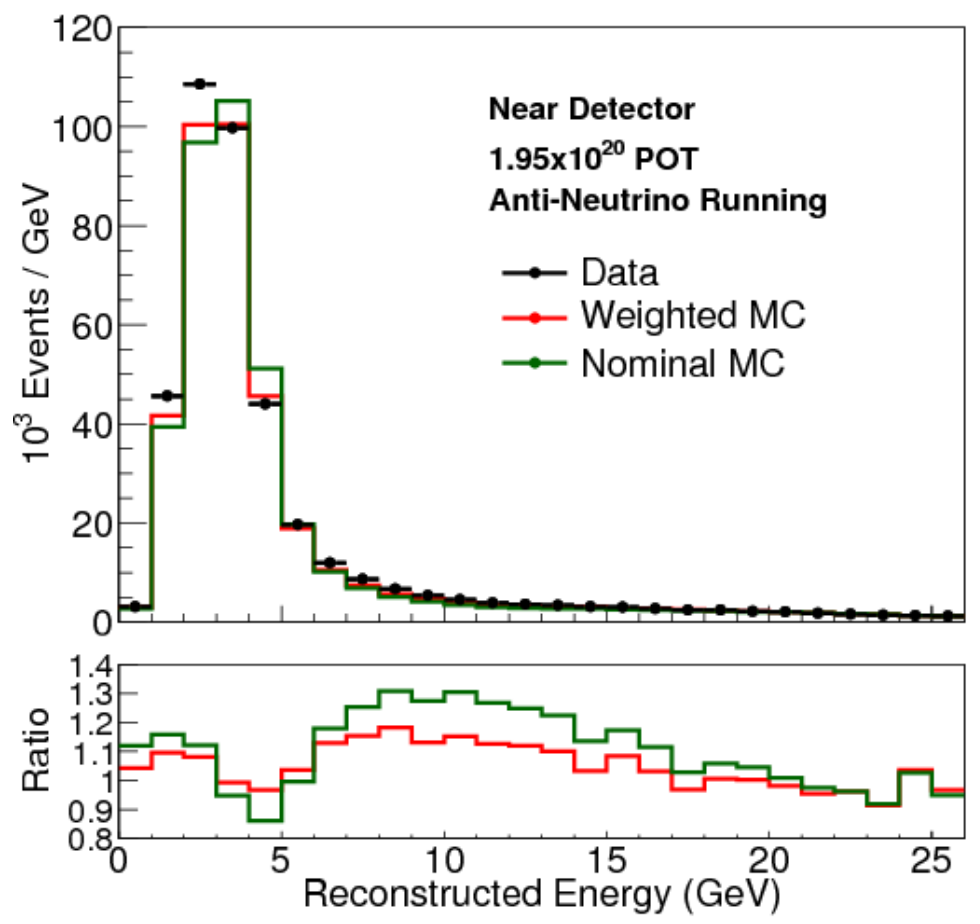

Figure 4.11: Data (black dots), untuned (green) and tuned (red) Monte Carlo comparison of the Near Detector antineutrino energy spectrum. 


\subsection{NEAR DETECTOR EVENT SAMPLE}

The near detector energy spectrum is obtained by summing the track and shower energies of selected events. The overall efficiency of this selector and contamination before and after selection is shown in Fig. 4.12. The sharp drop in efficiency is the result of the radical coil hole cut.

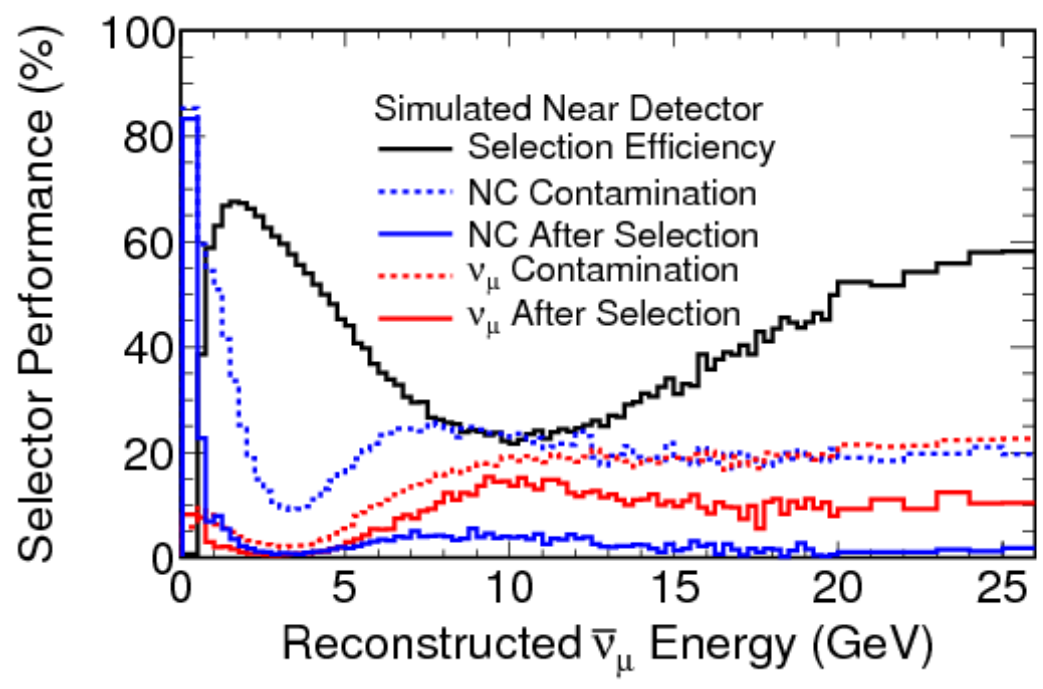

Figure 4.12: The black line shows the efficiency of the antineutrino selector, dashed lines show the CC wrong sign (red) and NC (blue) contamination before selection, solid colored lines show contamination after selection.

Fig. 4.13 shows the distribution of track, shower and neutrino energies and the inelasticity distribution. The red band shows the Monte Carlo with a systematic error band calculated by adding the effect of each systematic uncertainty in quadrature. (Details of systematic uncertainties are given in Section 4.8). The band includes the effects of the uncertainty in the beam flux and neutrino cross sections, which mostly cancel between the two detectors and have a negligible impact on the results. The blue band shows the size of the total background consisting of mis-identified neutrino interactions and neutral-current events. The data shows 
good agreement with Monte Carlo and the disagreements are within limits of the systematic error.
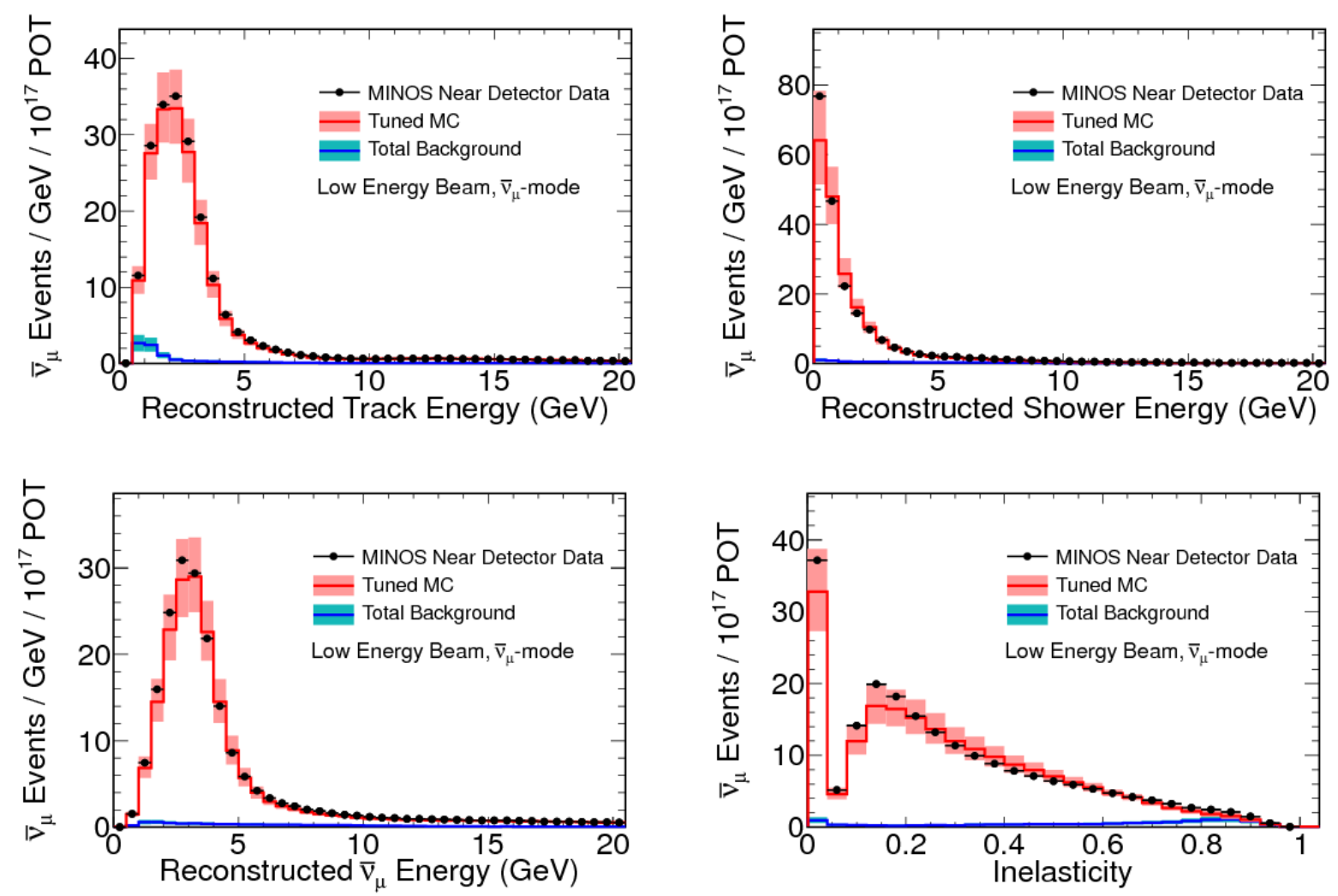

Figure 4.13: Plots show the distributions of track, shower and total antineutrino energy and inelasticity. Black dots represent ND data, red curve represents Monte Carlo where the pink band is the systematic error. The blue band shows the total background of neutral current and wrong-sign events. 
Fig. 4.14 shows the squared radius and longitudinal distributions of track vertices and ends. Fig. 4.15 shows the distributions of the $\mathrm{CC} / \mathrm{NC}$ separation variable and the charge/momentum ratio after the selection cuts are applied. Many other distributions including distributions of track shapes and sizes, kinematic variables, angular distributions and timing were investigated. Monte Carlo is seen to model data well.
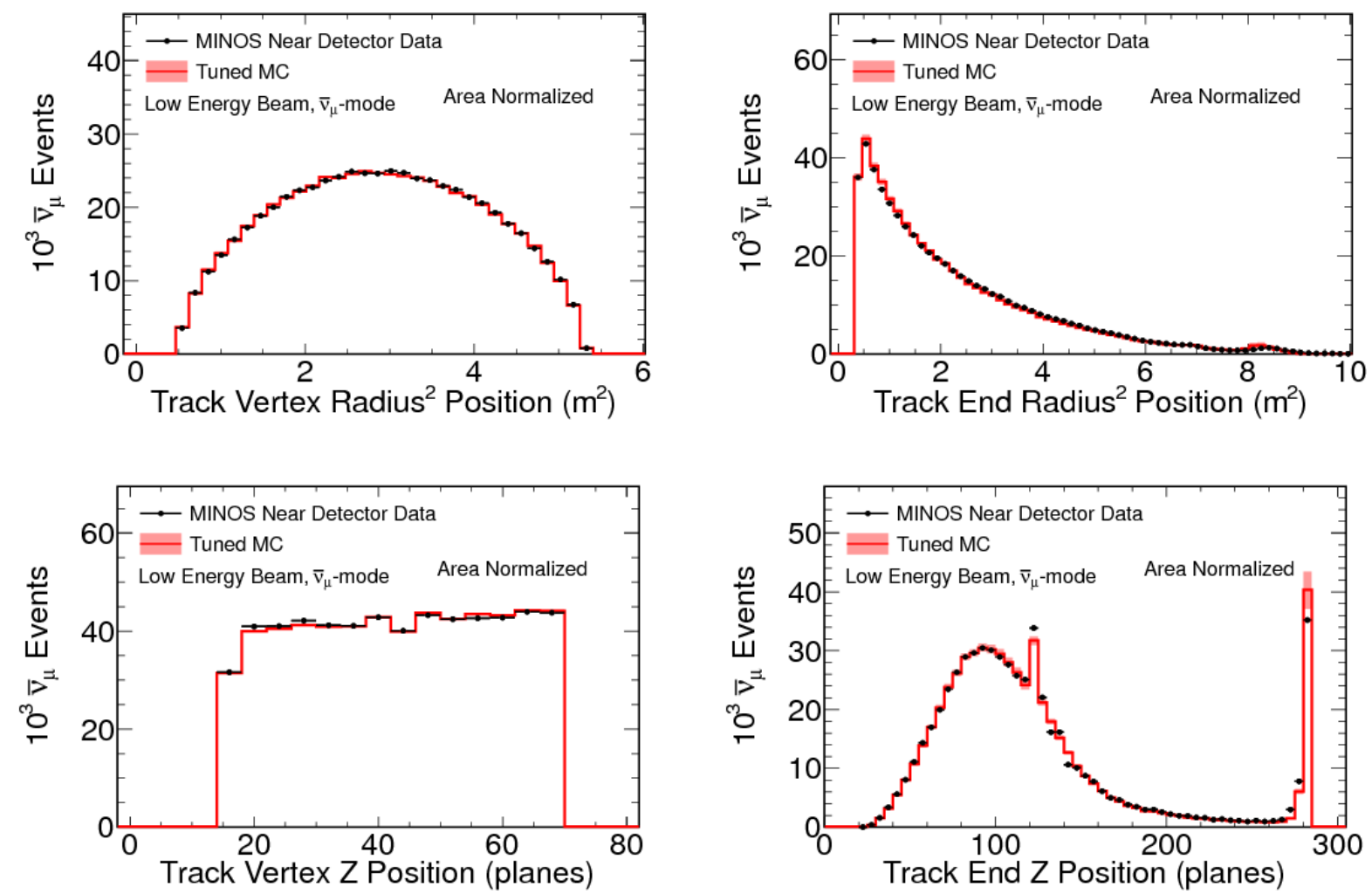

Figure 4.14: Top plots show the squared track vertex (left) and end radii in meters. Bottom plots show the longitudinal track vertex (left) and end positions in units of number of planes. Black dots represent ND data and the red curve represents Monte Carlo. Monte Carlo is area normalized to data to compare the shapes of the distributions independent of normalization. 

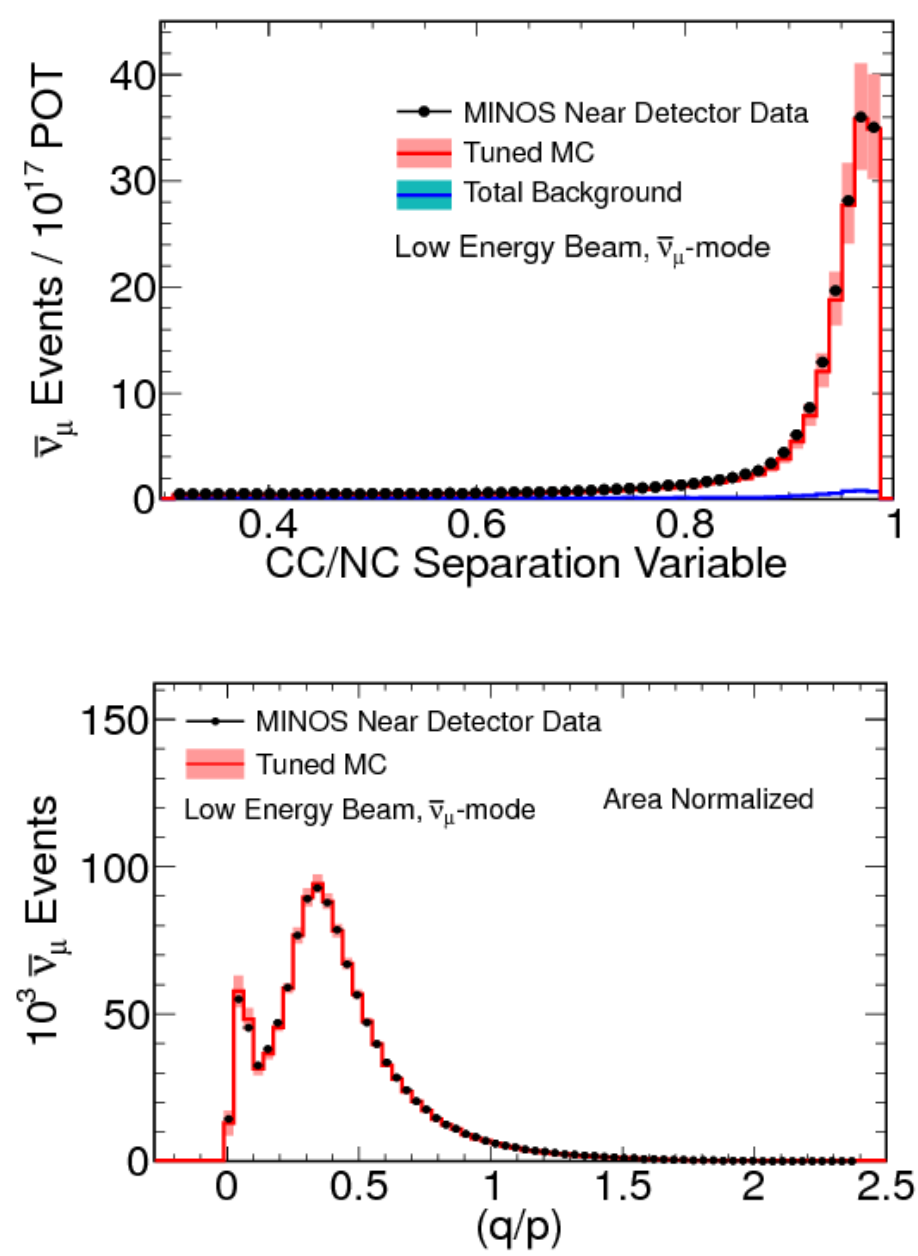

Figure 4.15: The top plot shows $\mathrm{CC} / \mathrm{NC}$ separation parameter of events passing all selection cuts. The bottom shows the charge/momentum ratio of all selected events. The red histogram represents the Monte Carlo expectation with systematic uncertainties, the blue histogram represents the total background with systematic uncertainties. Black points represent data. 


\subsection{NEAR TO FAR EXTRAPOLATION}

The Far Detector spectrum is not identical to the Near Detector even in the absence of oscillations because of the beamline geometry. Fig. 4.16 shows the beamline schematically. The Far Detector, $735 \mathrm{~km}$ away from the neutrino source, views a very narrow solid angle of the beam accepting only the most forward-going neutrinos. This constrains the decay angle and therefore the neutrino energy. The Near Detector views a much larger solid angle, accepting a wider range of decay angles and neutrino energies. Due to the proximity of the Near Detector to the Decay Pipe, the accepted solid angles also depend on the position of the decay along the pipe.

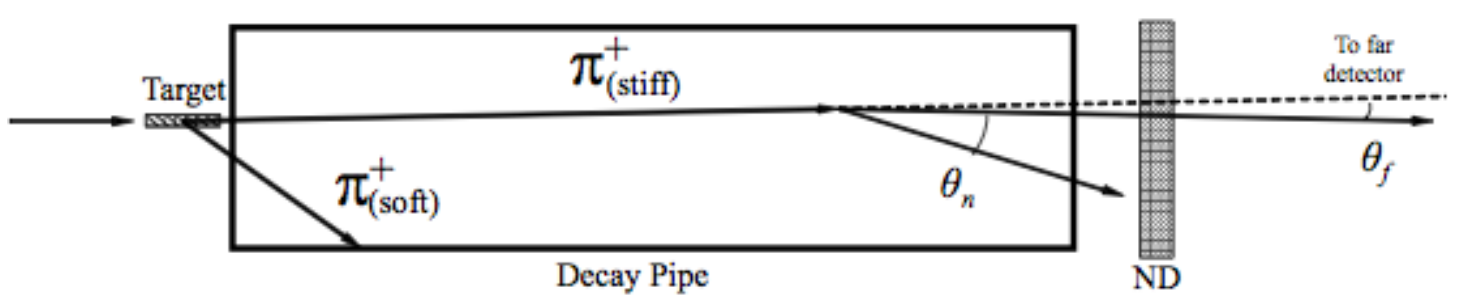

Figure 4.16: A cartoon of the upstream portion of the beamline where meson decay angles and angles accepted by the Near $\left(\theta_{n}\right)$ and Far $\left(\theta_{f}\right)$ are shown. The ND accepts a wider range of solid angles due to its proximity to the neutrino source than the FD, resulting in differences in beam spectra.

The ratio of the FD flux to the ND flux is shown in Fig. 4.17 and is clearly not flat which is the result of the above effects. The predicted spectra in the Near and Far Detector are shown in Fig. 4.18. The shaded colored regions indicate the energy spectra of neutrinos that the same parents produce in the Near and Far Detectors. The effect is larger at higher energies since higher energy parents travel further along the decay pipe before decaying, resulting in the ND's accepting the largest range of solid angles. The larger the solid angle for a given decay, the smaller the neutrino energy, so the effect of this phenomenon is to lower the ND neutrino energy while the FD accepts only the highest energy neutrinos. The greater 
Lorentz boost of higher energy parents narrows the range of solid angles in the laboratory frame, but does not compensate entirely for the effect.

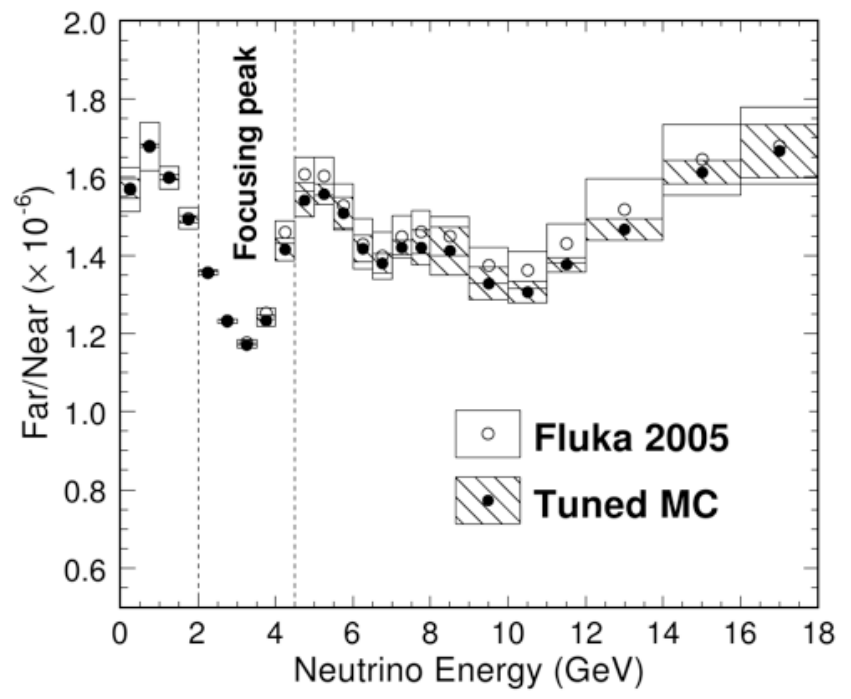

Figure 4.17: FD flux over the ND flux before extrapolation. The dashed bars show the tuned Monte Carlo and the empty bars are before tuning.
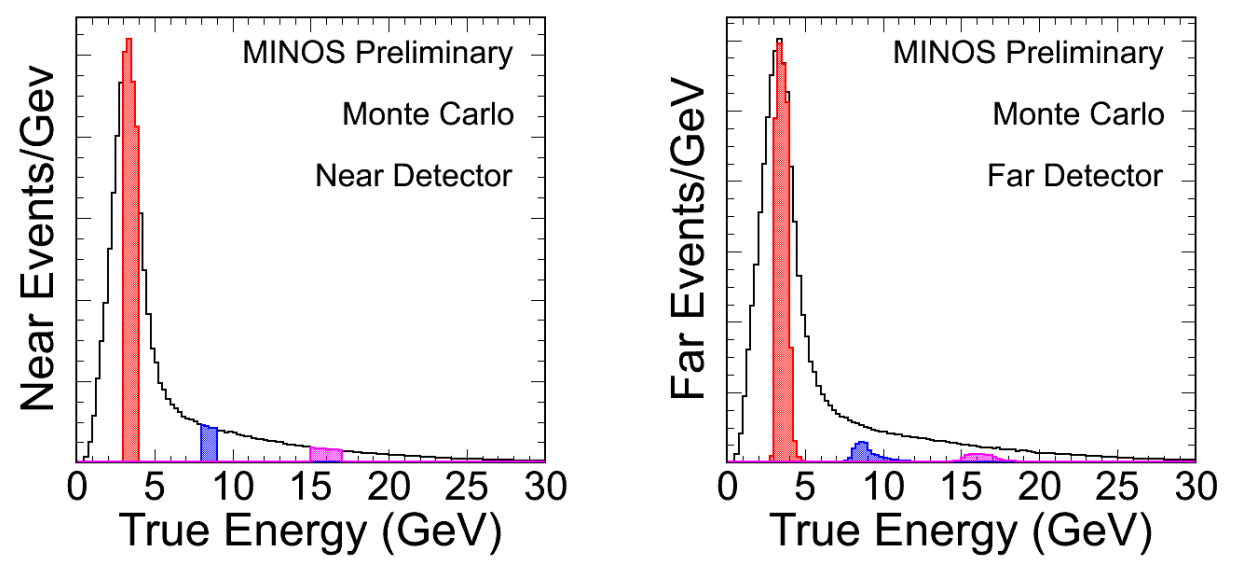

Figure 4.18: Simulated true energy spectra at the Near and Far Detectors. Colored shaded regions indicate the neutrino spectra that come from a given parent at the two detectors. 
The predicted Far Detector spectrum is obtained from the measured Near Detector spectrum by applying series of Monte Carlo based corrections to account for contamination, detector effects and effects due to the beam. A "beam matrix" transfers the ND flux to the FD. The flow chart in Fig. 4.19 outlines the extrapolation procedure.

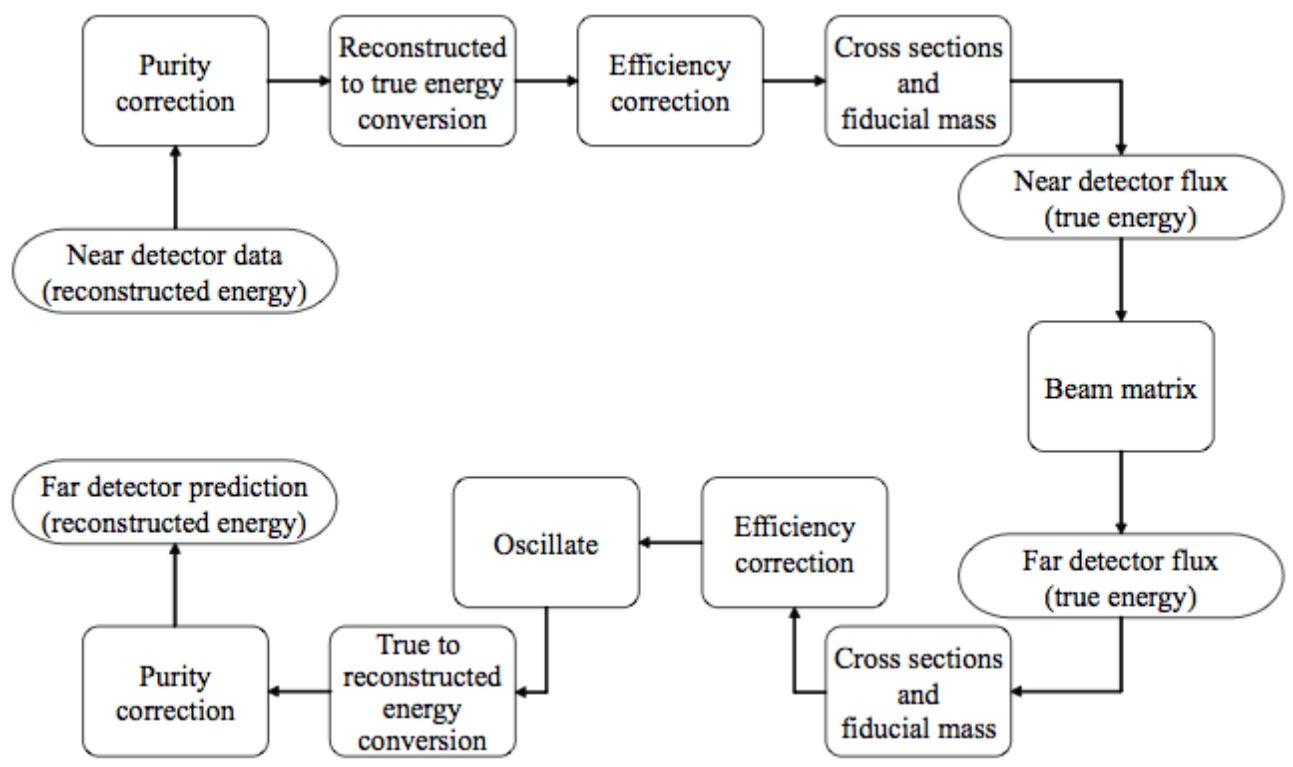

Figure 4.19: The steps of the beam matrix extrapolation method used to obtain FD predicted spectra from the measured ND spectra.

First, a purity correction is applied to the Near Detector energy spectrum to remove misidentified neutral-current events. Then, a (non-diagonal) matrix multiplies the puritycorrected spectrum to transform the reconstructed energy spectrum to a true energy spectrum. An efficiency correction is applied to the true ND energy spectrum; this removes the effect of selection cuts and reconstruction inefficiencies. The result is a true energy spectrum of all neutrinos that interacted in the Near Detector fiducial volume. This spectrum is divided by the energy dependent charged-current interaction cross-section and the fiducial volume, converting the energy spectrum into the ND flux.

Once the ND flux is obtained a matrix is generated to transfer it to the FD flux. Each parent in the simulation is made to decay towards a random interaction vertex in the Near Detector (this accounts for the ND acceptance of the neutrinos). The same parent is also 
made to decay towards an interaction vertex at the FD, though this time the selection of a random vertex is not necessary since all decays from which a FD neutrino emerges are along the $z$-axis. With this procedure a neutrino energy at the ND is related to a corresponding neutrino energy at the FD through their common parent. Repeating this procedure for every parent in the simulation, a beam matrix is computed as shown in Fig. 4.20. Applying this matrix to the ND flux yields the Far Detector flux.

In order to convert this Far Detector flux to a reconstructed energy spectrum, corrections and matrices similar to the ones which converted the ND energy spectrum to a flux are applied in reverse order. The flux is multiplied by the interaction cross-section and the FD fiducial volume. The efficiency correction is divided out to reintroduce the selection cuts and effects of reconstruction. Oscillations are applied to the energy spectrum as a function of true neutrino energy. The true energy spectrum is multiplied by a (non-diagonal) matrix to convert it to the reconstructed energy spectrum. Impurities from mis-identified neutralcurrent events are reintroduced. The procedure extrapolates neutrinos and antineutrinos separately which allows to account for differences in cross sections and the relative sizes of backgrounds.

Another way to obtain a far detector prediction from the near detector data is called the "Far/Near" method and simply multiplies the near detector data spectrum by the ratio of the far to near Monte Carlo spectra in bins $i$ of energy $E_{i}$ :

$$
\operatorname{FD}_{P r e d, i}\left(E_{i}\right)=\mathrm{ND}_{\text {Data }, i}\left(E_{i}\right) \cdot \frac{\mathrm{FD}_{M C, i}\left(E_{i}\right)}{\mathrm{ND}_{M C, i}\left(E_{i}\right)}
$$

This method is used as a cross-check to the analysis and gives very similar results. 


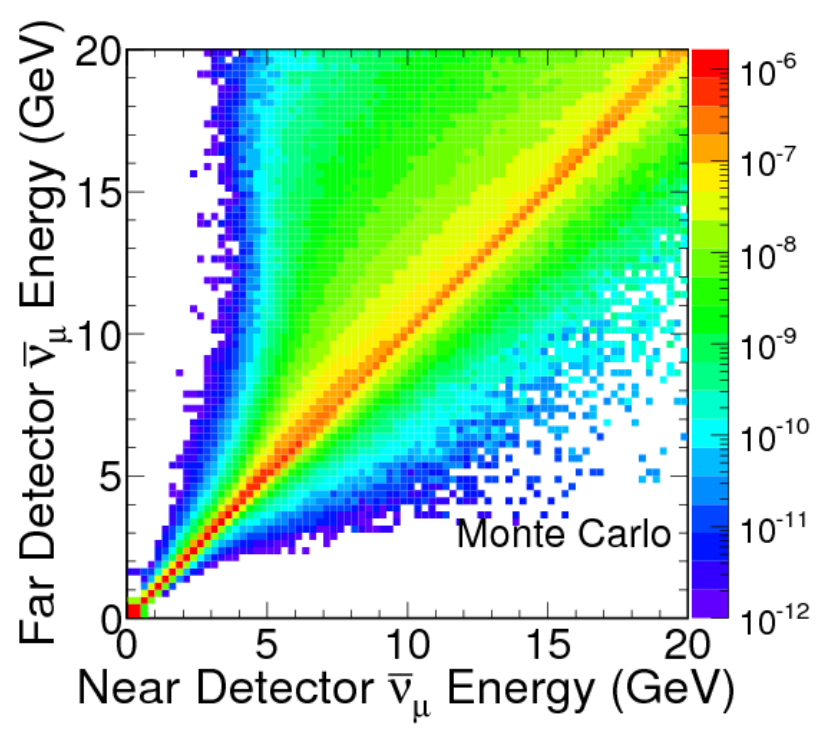

Figure 4.20: The beam matrix used in this analysis relates the antineutrino flux in true energy in the near detector to the far detector.

\subsection{SYSTEMATIC UNCERTAINTIES}

There are a number of systematic uncertainties that can affect the measurements of antineutrino oscillation parameters by altering the observed neutrino energy spectrum, the number of selected antineutrino events or the estimate of the background. The largest sources of uncertainties are muon energy scale, hadronic energy scale, neutral current background, normalization and overall cross section uncertainty.

\subsubsection{Muon energy scale}

The energy of muons stopping in the detector is measured from range. Uncertainty in this quantity comes from the knowledge of and variation in steel density and from modeling of energy loss in iron. This uncertainty is evaluated by comparing simulation to the CalDet

response and determined to be $2 \%$ by $[95,96]$. If a muon exits the detector its energy is 
measured by the curvature of the track in the magnetic field. The uncertainty from measuring the muon momentum from curvature is evaluated by comparing range and curvature measurements of stopping tracks in [71]. The $2 \%$ uncertainty in the range measurement therefore applies to curvature measurements. The additional uncertainty of the curvature measurement is estimated to be $1 \%$. Range and curvature uncertainty measurements are combined to give a total uncertainty of $3 \%$, taken to be fully correlated between the near and far detectors.

\subsubsection{Hadronic energy scale}

Section 3.1 details the reconstruction of hadronic showers and the two methods available: calorimetry and the $k \mathrm{NN}$ shower energy estimator. This analysis employs the $k \mathrm{NN}$ estimation method to measure the shower energy. While the $k \mathrm{NN}$ based method reduces the uncertainty, this analysis uses the calorimetric uncertainty estimate. This slightly conservative estimate covers the $k \mathrm{NN}$ shower energy uncertainty across all energies as shown in Fig. 4.21.

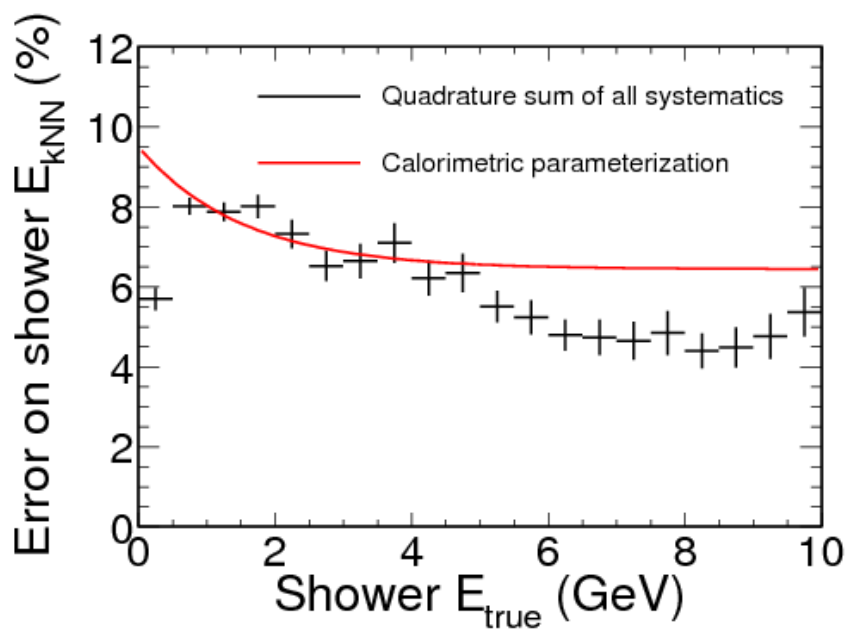

Figure 4.21: Black dots show systematic uncertainties on the kNN shower energy added in quadrature, the red line is the calorimetric parametrization. This analysis takes the calorimetric parametrization as the hadronic energy scale uncertainty.

The detector response to a hadronic shower is converted to a measured hadronic energy 
by the calibration procedure described in Section 3.2. There are two ways in which this conversion may be erroneous, and thus two sources of uncertainty on the shower energy measurement: a relative uncertainty from the difference in the calibration between Near and Far detectors, and an absolute uncertainty in the conversion factors of detector response to hadronic energy.

The relative uncertainty is accounted for by evaluating the difference between data and simulation at various steps of calibration and determined to be $1.85 \%$ in the Near Detector and $1.05 \%$ in the Far. The relative uncertainty is fully uncorrelated between the detectors.

The absolute uncertainty has two components. First, there is a $5.7 \%$ uncertainty in the conversion of CalDet response to hadronic shower energy. Second, there are uncertainties arising from modeling the hadron production. The size of this uncertainty is energy dependent since the models' performance varies across energy ranges. Adding the 5.7\% from CalDet response to this energy dependent in quadrature, the total uncertainty on the shower energy can be parametrized as [97],

$$
\sigma_{\text {had }}=6.6 \%+3.5 \% \times \exp \left(\frac{-E_{\text {had }}}{1.44 \mathrm{GeV}}\right)
$$

\subsubsection{Relative normalization}

The relative near/far normalization uncertainty consists of several components summarized in [98]. The three largest contributions to this uncertainty come from near/far selection bias $(1.3 \%)$, near detector fiducial bias $(0.7 \%)$ and far detector livetime $(0.32 \%)$. The near/far

selection bias uncertainty comes from the difference between Near and Far Detector reconstruction efficiencies. The double ratio,

$$
R=\frac{\epsilon_{D a t a}^{F D} / \epsilon_{M C}^{F D}}{\epsilon_{D a t a}^{N D} / \epsilon_{M C}^{N D}}
$$

where $\epsilon$ is the reconstruction efficiency would be equal to 1.0 if there were no modeling differences. The size of this uncertainty was evaluated by a manual scan of thousands of events. Scanners looked for missed tracks, miscalculated energies, fake tracks, charge misidentification and general reconstruction pathologies. The ND fiducial bias uncertainty 
accounts for differences in agreement between data and Monte Carlo in different regions of the ND fiducial volume. This is done by dividing the fiducial volume into two along $x, y$, and $z$ directions and evaluating the asymmetry in the ratio of data to Monte Carlo between halves. Far detector livetime uncertainty accounts for the far detector inefficiency quantified by the difference between POT counts obtained by two methods; $0.32 \%$ is the difference between POTs from data files and POTs from the database. Table 4.1 shows the components of the Near/Far normalization uncertainty.

\begin{tabular}{l|c}
\hline Systematic & Contribution to relative uncertainty (\%) \\
\hline \hline Steel thickness & 0.2 \\
Scintillator thickness & 0.2 \\
FD livetime & 0.32 \\
ND fiducial bias & 0.70 \\
N/F selection bias & 1.3 \\
\hline Sum in quadrature & 1.54 \\
\hline
\end{tabular}

Table 4.1: Contributions to the relative near/far normalization uncertainty, taken from [98].

\subsubsection{Backgrounds}

The uncertainty in neutral-current (NC) background modeling arises primarily from hadronic shower modeling. The systematic uncertainty on NC background was evaluated by comparing data to $\mathrm{MC}$ events in the $\mathrm{NC}$-dominated region (the region of $k \mathrm{NN}_{\mathrm{ID}}<0.3$ ). If the entire disagreement between data and Monte Carlo is attributed to the NC background, a $20 \%$ uncertainty covers the discrepancy.

There is an uncertainty associated with true neutrino events that are mis-identified as antineutrinos due to a charge sign measurement error. This mis-identified component is dominant for events with a large error in the $q / p$ measurement, which is quantified as $\sigma(q / p) /(q / p)>2.3$, where $\sigma(q / p)$ is the uncertainty on the $q / p$ measurement. To evaluate the size of this systematic the entire discrepancy between data and simulation in this 
subset of events is attributed to mis-identified charge sign. A maximum scale of $\pm 30 \%$ is required to make data and $\mathrm{MC}$ agree as a function of energy, so an uncertainty of $30 \%$ was taken on this background component.

\subsubsection{Cross section uncertainties}

There are four components that go into the cross-section uncertainty: quasi-elastic, resonance, deep-inelastic scattering (DIS) and total cross-section.

- Quasi-elastic and Resonance cross-section uncertainty. MINOS models the cross sections in this regime by the axial-vector masses $M_{A}^{Q E}$ and $M_{A}^{R e s}$. Taking into account the uncertainty in the data used to obtain the best information on these parameters and nuclear effects, a $\pm 15 \%$ error is estimated on $M_{A}^{R e s}$ and $\pm 30 \%$ on $M_{A}^{Q E}$.

- Total cross section. Fits to world $\mathrm{CC} \nu_{\mu}$ cross section data in the $10-30 \mathrm{GeV}$ range yield an estimate of $3.5 \%$ for normalization of the overall CC interaction cross section.

- Cross sections in the Resonance/DIS Transition Region. This uncertainty comes from the uncertainty on the parameters of the neutrino event generator, NEUGEN. The error is estimated by a combination of fits to experimental data and variations in the fit with changes in the interaction model parameters.

The uncertainties are evaluated by varying the parameters in the cross section model. Antineutrino cross sections have larger uncertainties so an additional set of uncertainties is considered only for antineutrinos. Details of cross-section uncertainties are given in [99].

\subsubsection{Smaller sources of systematic uncertainties}

Beam modeling The beam modeling uncertainty arises primarily from uncertainties in the hadron production model as well as beam optics, horn current, horn position, target position, and the shape and amount of material in the horns. The total error that results has been evaluated in [100] for each neutrino run period and adapted for antineutrino-mode running. Beam modeling uncertainties cancel nearly entirely between the two detectors because the 
beam is measured at the Near Detector and the Far Detector prediction is obtained from this.

Near Detector Acceptance In order to evaluate the uncertainty on Near Detector acceptance which was not included in previous oscillation analyses, a special run was performed operating the Near Detector coil in reverse polarity so that $\mu^{+}$tracks from antineutrino interactions are de-focused. The ratio of Far Detector predictions obtained from regular, focused ND data and this special de-focused data provides an error band that is incorporated as an uncertainty in this analysis.

\subsection{FAR DETECTOR SELECTION}

The Far Detector sample is selected by the $\mathrm{CC} / \mathrm{NC}$ separation and charge sign selection as the ND. In order to remove contamination from cosmic muons, tracks are required to have at most an angle $\theta$ with the beam axis such that $\cos \theta<0.6$. The overall efficiency of this selector and contamination before and after selection at the Far Detector is shown in Fig. 4.22 .

This is a blind analysis, meaning any far detector data spectrum that holds the possibility of revealing the magnitude of oscillations is not studied until the entire analysis chain is finalized. Since oscillations will manifest as a disappearance in the far detector data, these spectra are handled with extra care. It is still necessary to check that data is well modeled by the Monte Carlo simulation. To accomplish this we only look at far detector distributions of variables that are insensitive to oscillations, such as spatial distributions, and the overall normalization of the event count is hidden. In order to study oscillation-sensitive distributions, such as energies and kinematic variables, a blinding algorithm is applied to data which removes a subset of the events from the dataset in a way that will mask the effect of oscillations. After the analysis is finalized, unblinded and events are counted, the properly normalized versions of these comparisons are performed with the full dataset.

With $2.95 \times 10^{20}$ POT of exposure, a total of 273 antineutrino events are expected at the Far Detector in the case of no oscillations and 193 antineutrino events are observed. 


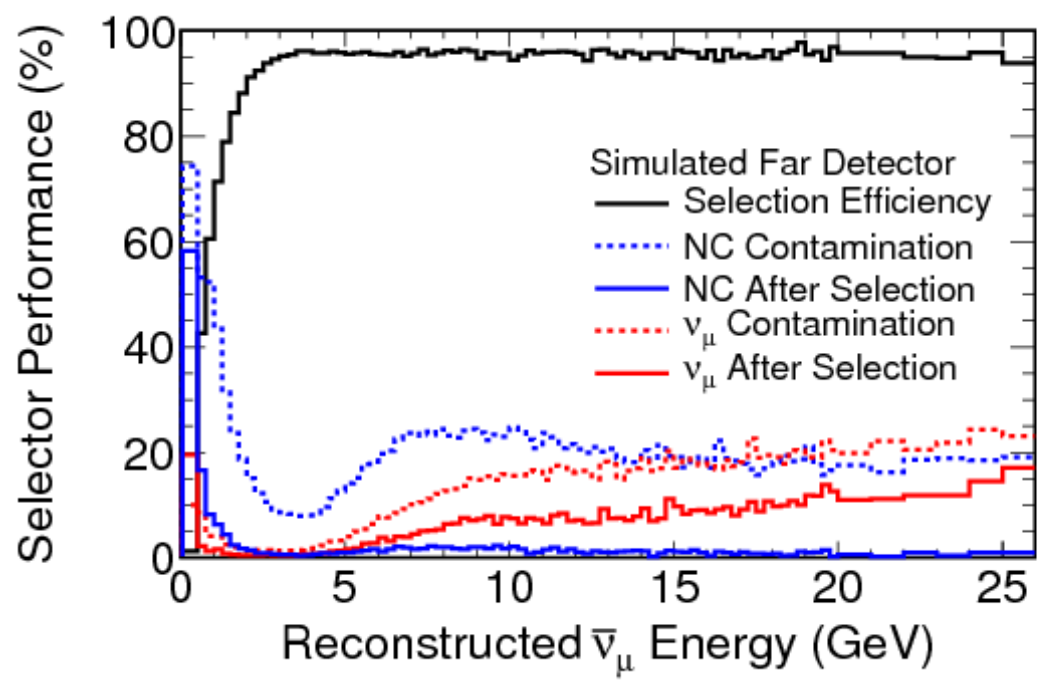

Figure 4.22: The black line shows the efficiency of the antineutrino selector, dashed lines show the CC wrong sign (red) and $\mathrm{NC}$ (blue) contamination before selection, solid colored lines show contamination after selection.

Fig. 4.23 shows the neutrino energy distribution of the predicted spectrum in the absence of oscillations and FD data. The energy dependent disappearance is clearly visible. Error bars assume a Poisson-distribution in bins of less than 50 events via the prescription in [101] They are Gaussian for bins with more events.

Fig. 4.24 shows the spatial distribution of track vertices and ends in the detector. As expected, interaction vertices are distributed randomly and tracks curve towards the coil hole due to the magnetic field. Fig. 4.26 shows the track and shower energy distributions. Fig. 4.25 shows the squared radius and longitudinal vertex and end positions. Fig. 4.27 shows the distribution of the angle between tracks and the beam axis. The simulation is shown with no oscillations and at the best fit oscillation values (the fit is detailed in the next section). Data is well modeled by the simulation and the energy-dependent disappearance due to oscillations is clearly visible in these spectra. 


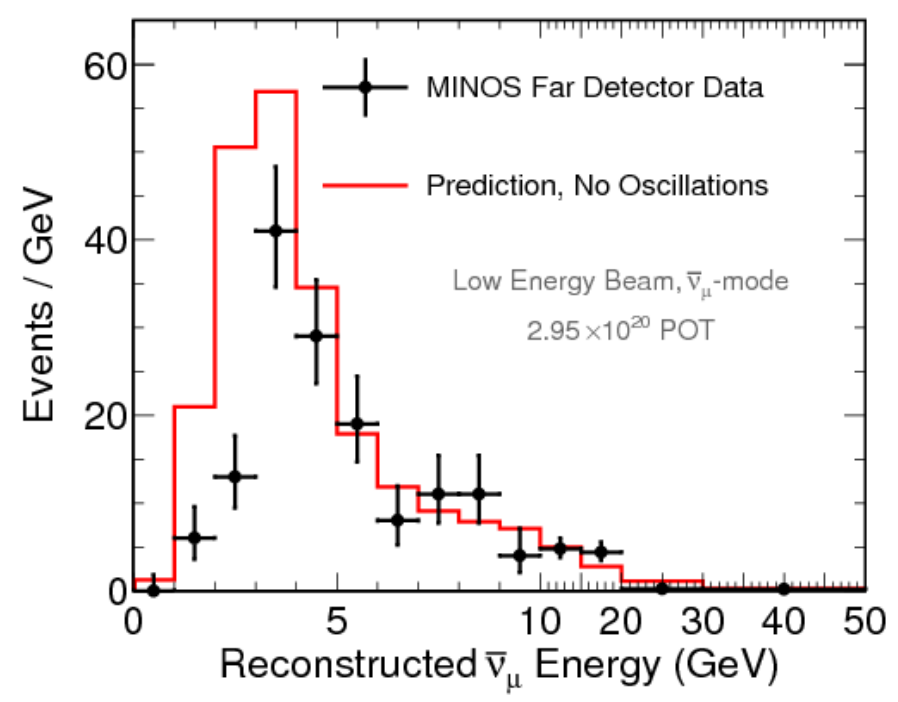

Figure 4.23: Antineutrino energy distribution of selected FD events (black dots) and the prediction in the absence of oscillations. Error bars are Poisson.
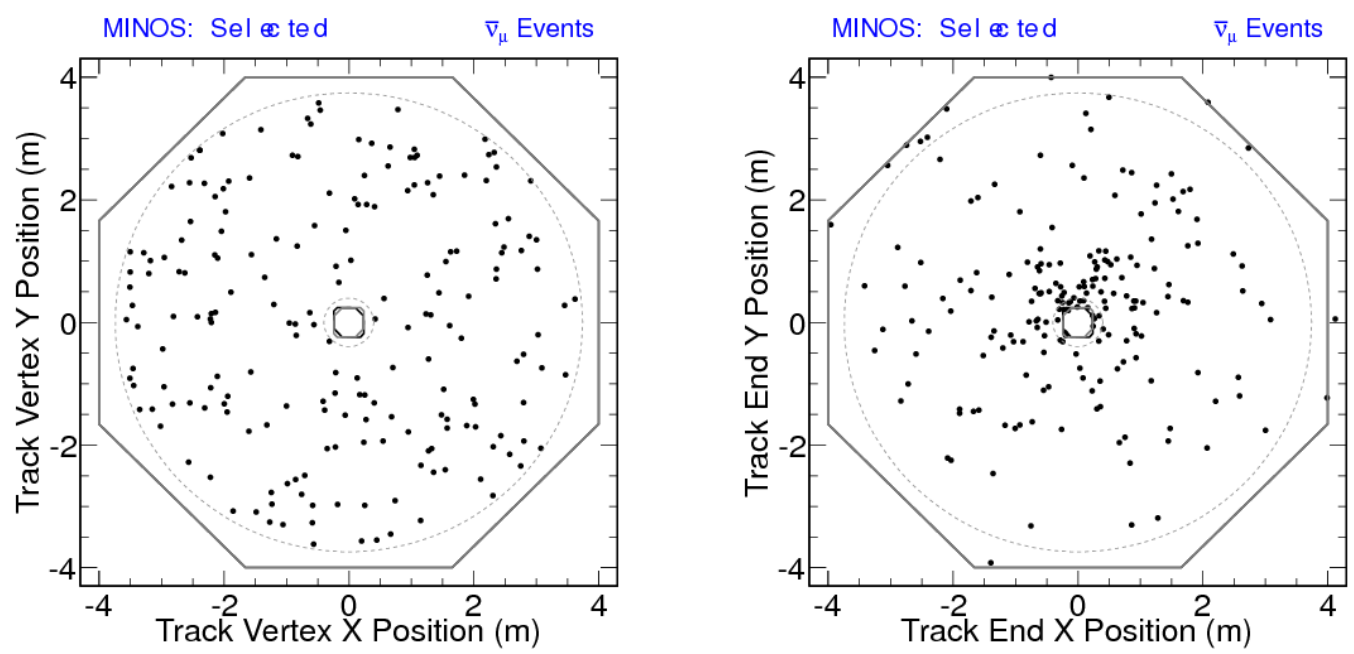

Figure 4.24: $y$ vs. $x$ distribution of antineutrino interactions in the Far Detector. 

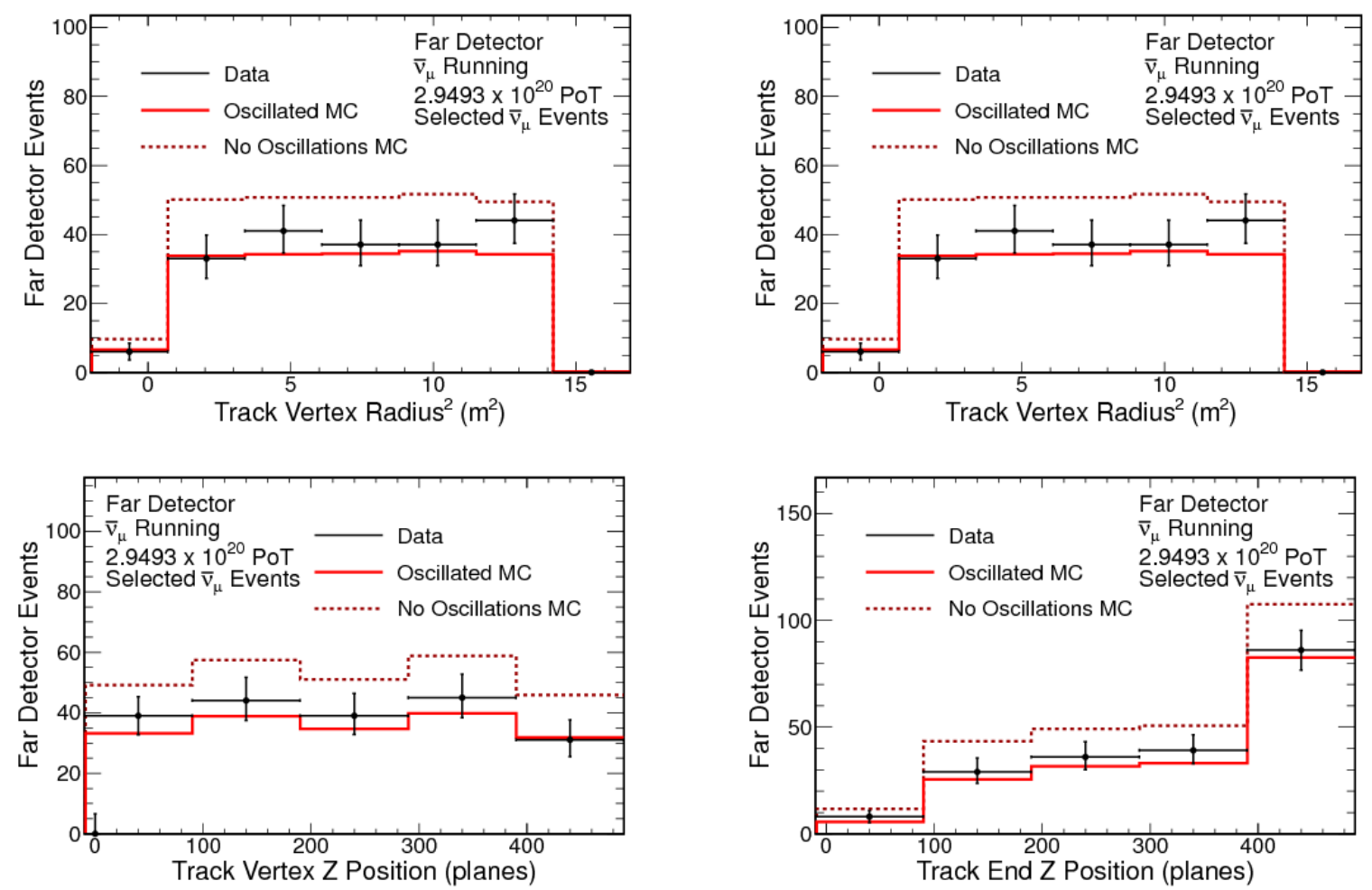

Figure 4.25: Top plots show the squared track vertex (left) and end radii in meters. Bottom plots show the longitudinal track vertex (left) and end positions in units of number of planes. Black dots represent FD data, the solid red curve represents Monte Carlo with oscillations and dashed curve is without oscillations. 

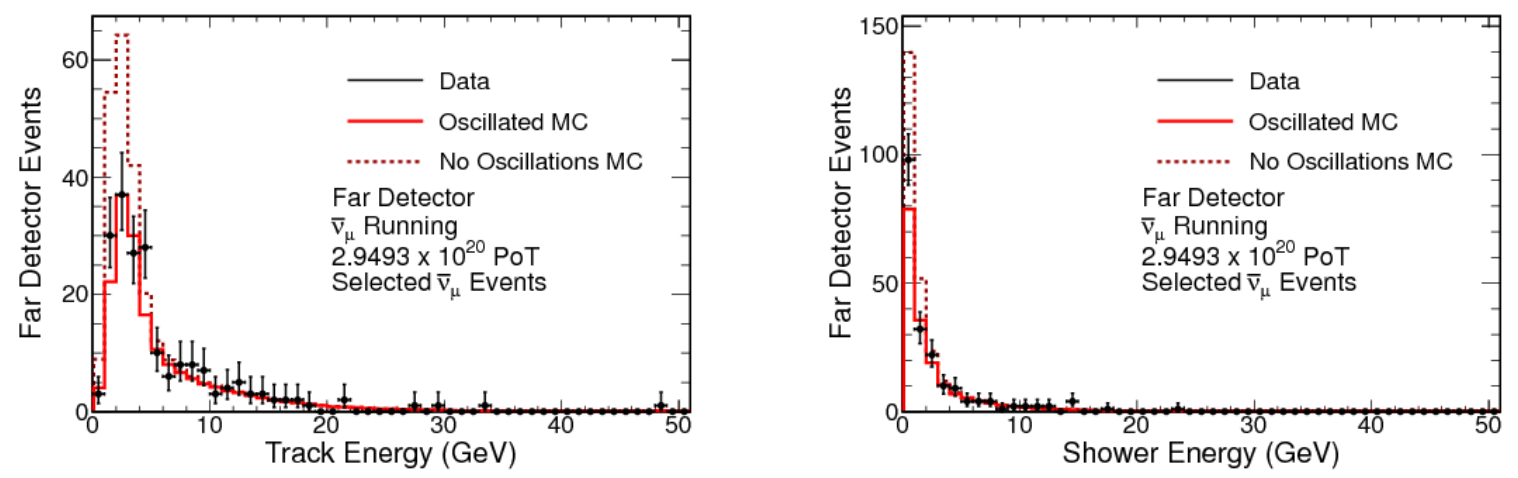

Figure 4.26: Muon track and shower energy distributions of selected antineutrino events in the Far Detector. The dashed red line shows the predicted spectrum in the absence of oscillations, the solid red line is the prediction at the best fit and black dots show data with Poisson error bars.

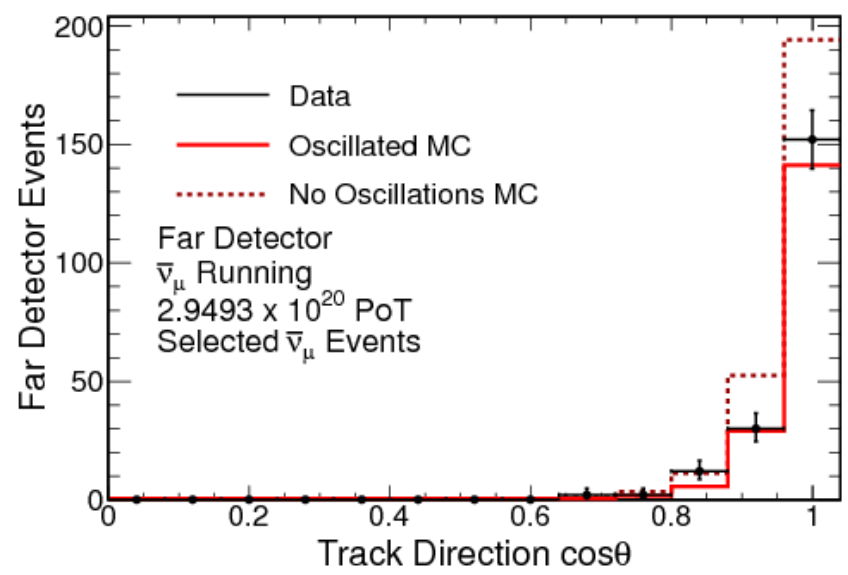

Figure 4.27: Distribution of the cosine of the angle between tracks and the beam axis. Events with $\cos \theta<0.6$ are excluded. Black dots represent FD data, the solid red curve represents Monte Carlo with oscillations and dashed curve is without oscillations. 


\subsection{FITTING THE DATA}

The measured far detector energy spectrum is fit to the two flavor vacuum oscillation model from Section 1.3, in which the disappearance probability of a muon neutrino is given Eq. (4.9) by,

$$
P_{\bar{\nu}_{\mu} \rightarrow \bar{\nu}_{\mu}}=\sin ^{2} 2\left(\bar{\theta}_{23}\right) \sin ^{2}\left(1.27 \frac{\Delta \bar{m}_{32}^{2} L}{E}\right) .
$$

The best fit to data is calculated by minimizing the standard log-likelihood function between data and predictions generated over a grid of $\Delta \bar{m}^{2}, \sin ^{2} 2 \bar{\theta}$ sets as shown in Eq. (4.10). The fit is performed in 100 bins, $i$, for each run period $r$. The value of the mixing angle is constrained to be physical, that is, the allowed range is $0<\sin ^{2}(2 \theta)<1$.

$$
-2 \ln \lambda=2 \sum_{r=1}^{2} \sum_{i=1}^{100} n_{r i}^{\text {pred }}-n_{r i}^{\text {data }} \ln \frac{n_{r i}^{\text {data }}}{n_{r i}^{\text {pred }}}
$$

Confidence intervals and allowed regions in $\left(\Delta \bar{m}^{2}, \sin ^{2} 2 \bar{\theta}\right)$ space are computed by using the Feldman-Cousins (FC) method $[102,103]$. The $\Delta \chi^{2}$ that corresponds to a certain Gaussian coverage is known analytically from statistics [18]. The FC method is a frequentist approach where this coverage is calculated by running a large number of pseudo-experiments at each point and determining what value of $\Delta \chi^{2}$ covers the desired percentage of experiments. There are three concerns that the use of the Feldman-Cousins prescription alleviates [68]. First, parameters are being measured near physical boundaries at $\sin ^{2} 2 \bar{\theta}=0,1$ and $\Delta \bar{m}^{2}=0$, where Gaussian confidence intervals are too conservative (over-coverage). Second, a high $\Delta \bar{m}^{2}$ affects the event rate in the same way as $\sin ^{2} 2 \bar{\theta} \approx 0.5$. There is a difference

in the low energy spectra these two effects produce but with a small dataset this difference is not resolved. This gives rise to a degeneracy which results in under-coverage if Gaussian confidence intervals are used. Finally, using the Feldman-Cousins method allows the effect of systematic uncertainties to be included in the confidence contours.

Each point on the likelihood surface corresponds to a $\left(\Delta \bar{m}^{2}, \sin ^{2} 2 \bar{\theta}\right)$ pair. To obtain the $\Delta \chi^{2}$ which corresponds to a certain coverage at a given point, a large number of pseudoexperiments are generated assuming that true non-standard oscillation parameters are at 
this point. 100,000 experiments per point were generated and their $\Delta \chi^{2}$ 's were computed. As an example, consider that we want to calculate the $90 \%$ confidence contour. With 100,000 experiments, the 90,000 th largest $\Delta \chi^{2}$ will give the $90 \%$ coverage for that point. Then, the contour is determined by simply looking up the corresponding $\Delta \chi^{2}$ for a particular coverage at every point. In practice, this is done by filling a $90 \%$ Feldman-Cousins surface, where each point in this surface is the $\Delta \chi^{2}$ covering $90 \%$ of the experiments at that point. This surface is subtracted from the log-likelihood surface of the data fit; the contour is the collection zero points after subtraction. The surface for $90 \%$ confidence level coverage is shown in Fig. 4.28. The $\Delta \chi^{2}$ that corresponds to the $90 \%$ Gaussian confidence interval in a two parameter fit is $4.61[18]$. As expected, the Feldman-Cousins $90 \%$ coverage is lower than this near $\sin ^{2} 2 \bar{\theta}=1.0$ and higher everywhere else, with strongest signs of Gaussian under-coverage near high values of $\Delta \bar{m}^{2}$.

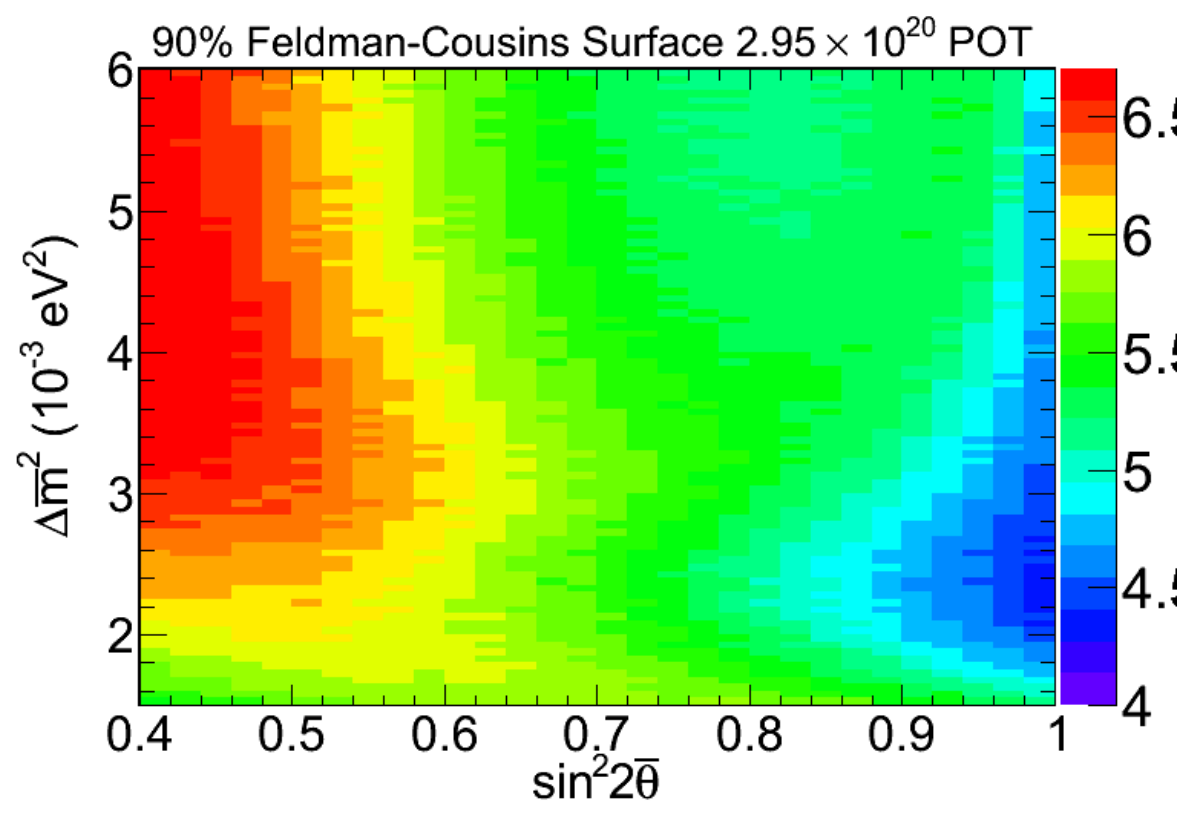

Figure 4.28: The Feldman-Cousins surface consisting of $\Delta \chi^{2}$ values corresponding to $90 \%$ coverage. Gaussian $90 \%$ coverage in two parameters is at $\Delta \chi^{2}=4.61$.

In order to account for the effect of systematic uncertainties, each pseudo-experiment is generated with a systematic shift whose value is drawn at random from a range of zero 
to maximum shift. Overall normalization, energy scale uncertainties, cross section uncertainties, flux modeling error, the acceptance uncertainty and background uncertainties are included in the pseudo-experiments. Fig. 4.29 compares Gaussian contours to FC corrected contours with and without the effect of systematics. The effect of systematics is small while the difference between Gaussian and Feldman-Cousins contours are non-negligible. The jaggedness of contours at high significance results from the finite number of pseudoexperiments; it is extremely CPU-intensive to generate the number of experiments required to achieve a smooth $99 \%$ (or higher) confidence interval.

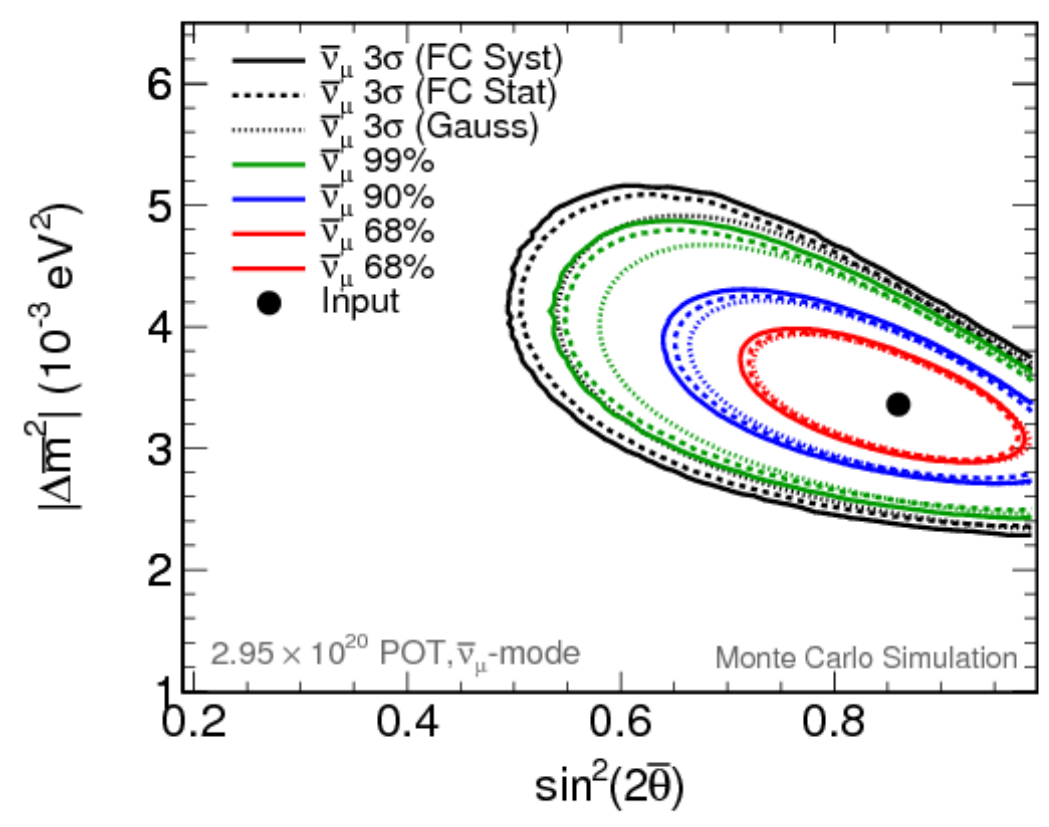

Figure 4.29: Comparisons of the effect of using the Feldman-Cousins method with and without systematic uncertainties, and Gaussian contours.

Fig. 4.30 shows the predicted Far Detector spectrum in the absence of oscillations (red), with the best oscillation fit values (blue) and data. The binning shown here is the display binning, the fit was performed in finer bins. 
The set of parameter values that best fit the data are

$$
\begin{aligned}
\left|\Delta \bar{m}_{\mathrm{atm}}^{2}\right| & =2.62_{-0.28}^{+0.31} \text { (stat.) } \pm 0.09 \text { (syst.) } \times 10^{-3} \mathrm{eV}^{2} \\
\sin ^{2}(2 \bar{\theta})_{23} & \left.=0.95_{-0.11}^{+0.10} \text { (stat.) } \pm 0.01 \text { (syst. }\right) \\
\sin ^{2}(2 \bar{\theta})_{23} & >0.75 \quad(90 \% \text { C.L. })
\end{aligned}
$$

The statistical errors on the above numbers are the $1 \sigma$ errors from the one-parameter Gaussian fits to each parameter. Note that the sign of $\left|\Delta \bar{m}_{\mathrm{atm}}^{2}\right|$ cannot be distinguished because its squared sine appears in the oscillation probability formula. The contours shown in Fig. 4.31 are obtained by subtracting the corresponding FC surfaces as explained above.

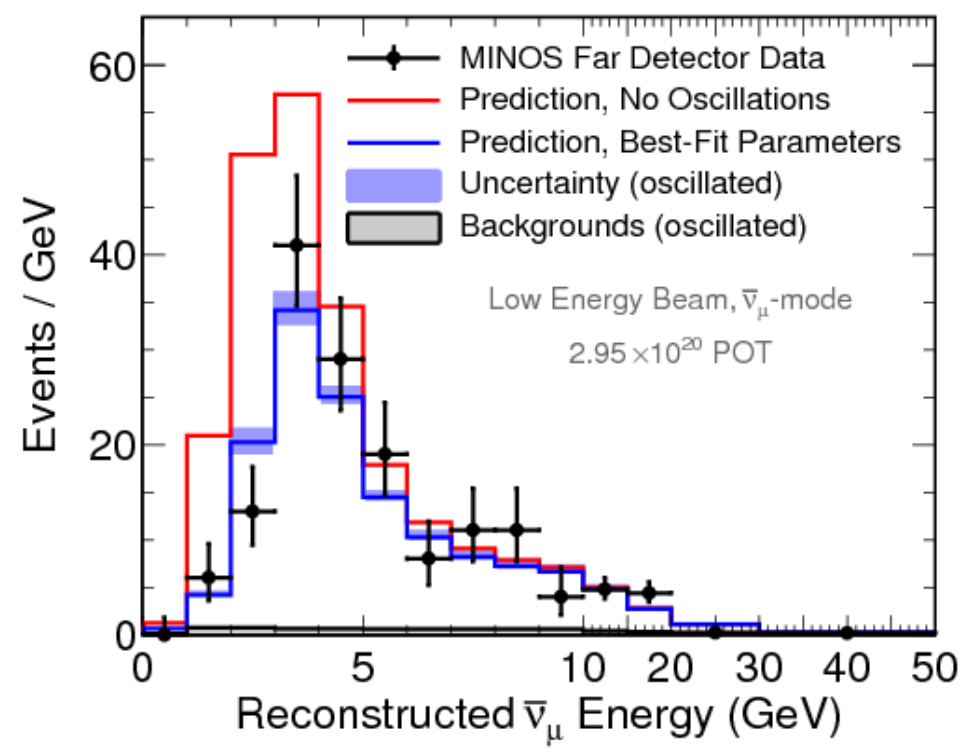

Figure 4.30: Far detector $\bar{\nu}_{\mu}$ data and predictions with no oscillations (red histogram) and with the best-fit oscillation parameters (blue histogram). The band around the oscillated prediction represents the total systematic uncertainty. Total background in the oscillated prediction is also displayed (gray shaded histogram). The data and predictions correspond to an exposure of $2.95 \times 10^{20}$ POT. 


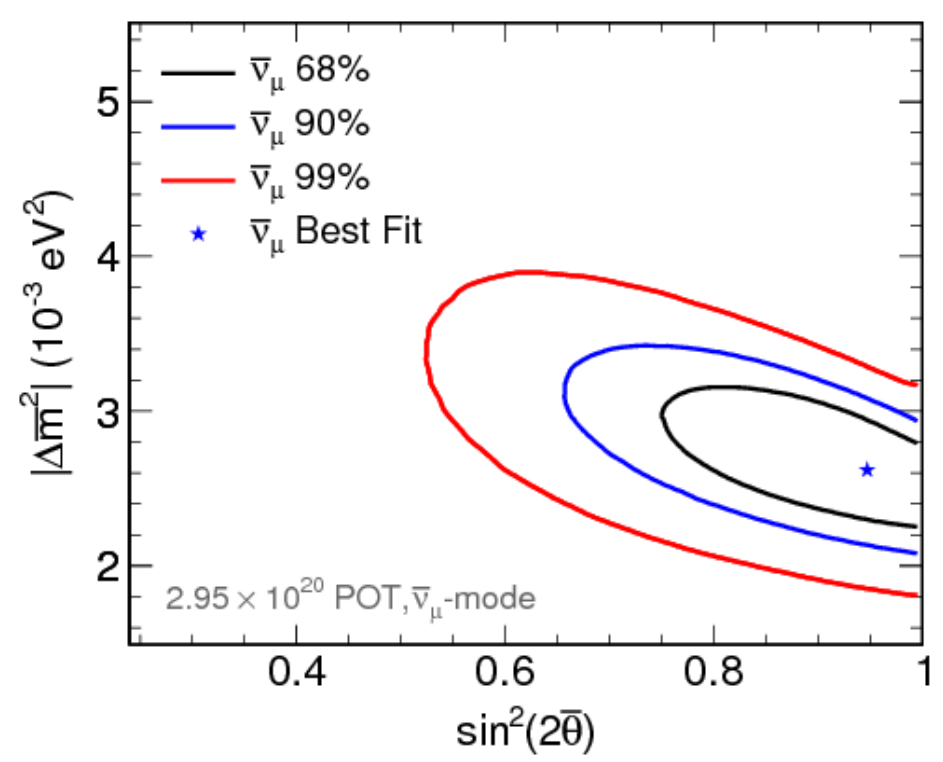

Figure 4.31: The $68 \%, 90 \%$, and $99 \%$ antineutrino oscillation contours. The contours are determined using the Feldman-Cousins method. The best fit is at $\left|\Delta \bar{m}_{\text {atm }}^{2}\right|=2.62_{-0.28}^{+0.31}$ (stat.) \pm 0.09 (syst.) $\times 10^{-3} \mathrm{eV}^{2}$ and $\sin ^{2}\left(2 \bar{\theta}_{23}\right)=0.95_{-0.11}^{+0.10}$ (stat.) \pm 0.01 (syst.) .

\subsubsection{Evaluation of Oscillation Parameter Uncertainties}

The effect of systematic uncertainties are calculated by generating a set of systematically shifted fake data for each run period, as well as an unshifted (nominal) set. Generated events are treated like real data and passed through the entire analysis chain to fit for oscillation parameters. In fitting these systematically shifted pseudo-datasets, the value of $\sin ^{2}(2 \theta)$ is not constrained to be physical. The difference between the best fit values of a shifted dataset to the nominal data set is calculated. Systematically shifted fake data were generated with oscillation parameters $\left|\Delta \bar{m}^{2}\right|=2.62 \times 10^{-3} \mathrm{eV}^{2}$ and $\sin ^{2}(2 \bar{\theta})=0.947$ [104]. Fig. 4.32 shows the effect of these shifts graphically. The largest systematic uncertainties in the mass-splitting measurement are muon and hadronic energy scale. For mixing angle the largest effect comes from Near/Far normalization. Tables 4.2 lists the best fits to nominal 
and shifted datasets and the differences between the two numerically.

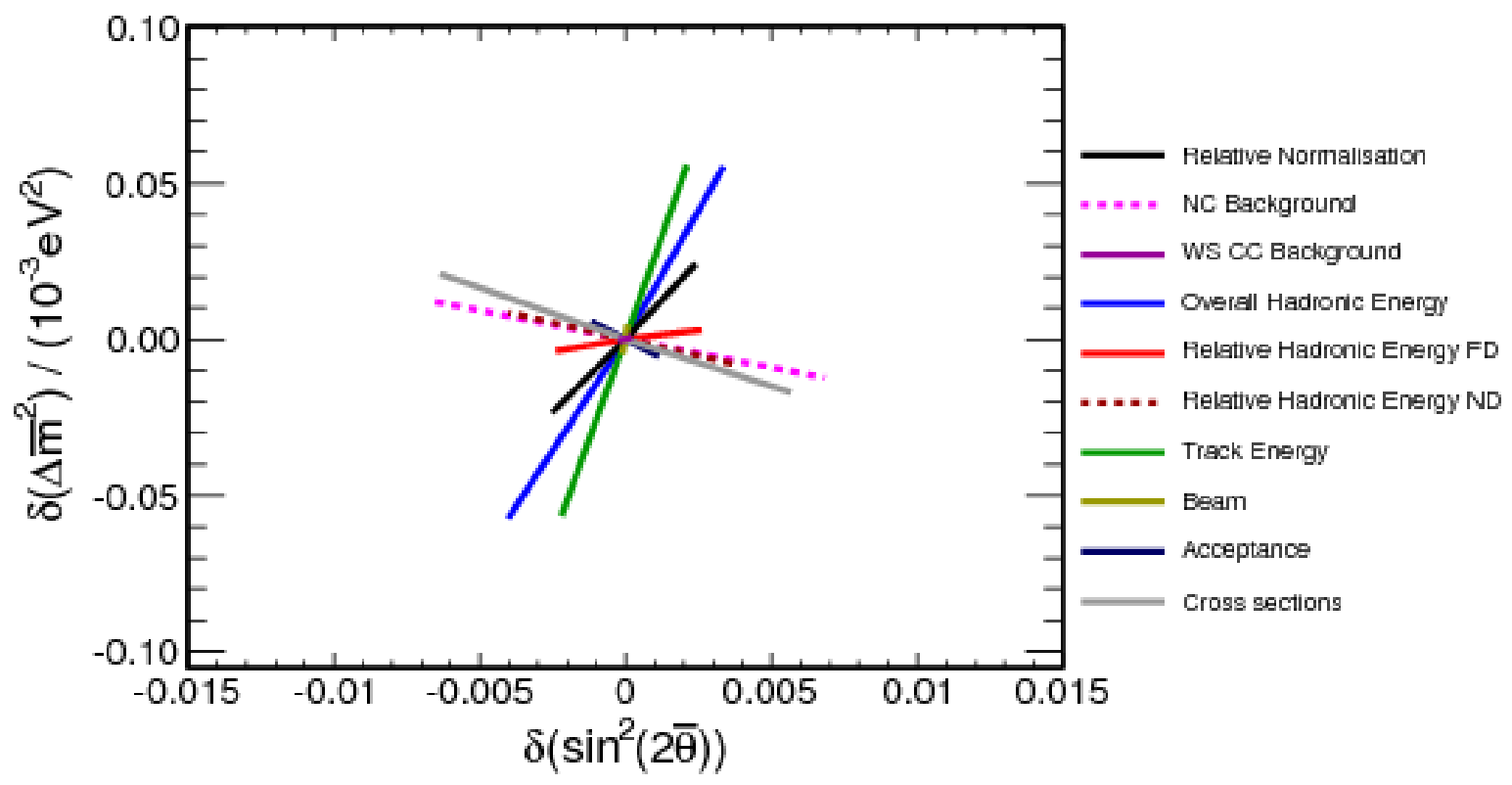

Figure 4.32: The deviations in the best fit parameters from their nominal positions when the analysis is performed on systematically shifted datasets.

To study the effect of systematic uncertainty on the number of predicted Far Detector $\bar{\nu}_{\mu}$ events, systematically shifted high statistics near and far detector fake datasets are created from Monte Carlo. A far detector prediction is generated from each systematically shifted near detector fake data set using identical oscillation parameters to the fake data. The prediction is scaled to $2.95 \times 10^{20}$ protons on target. To obtain the total fractional systematic error in the number of events at the far detector, the double ratio

$$
1-\frac{\text { Shifted FD Prediction / Shifted FD Fake Data }}{\text { Nominal FD Prediction / Nominal FD Fake Data }}
$$

is calculated for each systematic shift. The shifted far detector fake data encapsulates the effect of systematic uncertainties on the far detector. The shifted far detector prediction is obtained by extrapolating the shifted near detector fake data and thus reflects the systematics at the near detector. The above ratio therefore takes into account cancellations between the 
near and far detectors. In each energy bin, positive values of expression (4.13) are added in quadrature to obtain the upper and negative values to give the lower systematic error band. The resulting systematic error band is shown in Fig. 4.33.

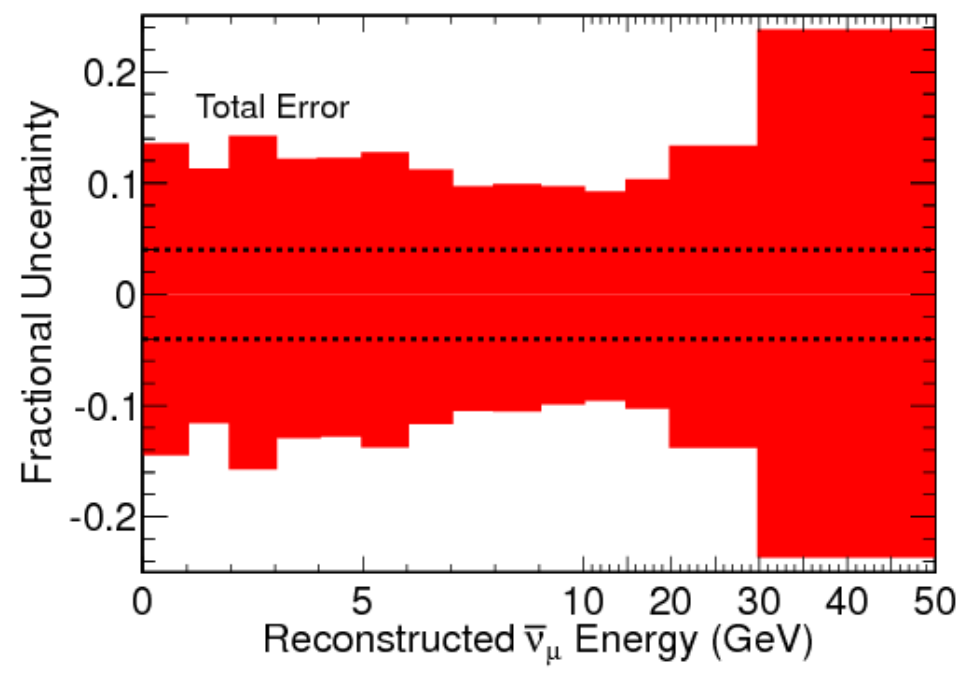

Figure 4.33: The total systematic error band on the number of predicted $\bar{\nu}_{\mu}$ events. The band is obtained by adding the effect of each individual systematic shift on the FD predicted energy spectrum in quadrature.

While systematic uncertainties have been treated with care and their effects taken into account in the fit, this is a highly statistics-limited analysis. Fig. 4.34 compares the size of systematic shifts to the statistical sensitivity. The statistical size of allowed regions completely cover the systematics which are miniscule on this scale. 


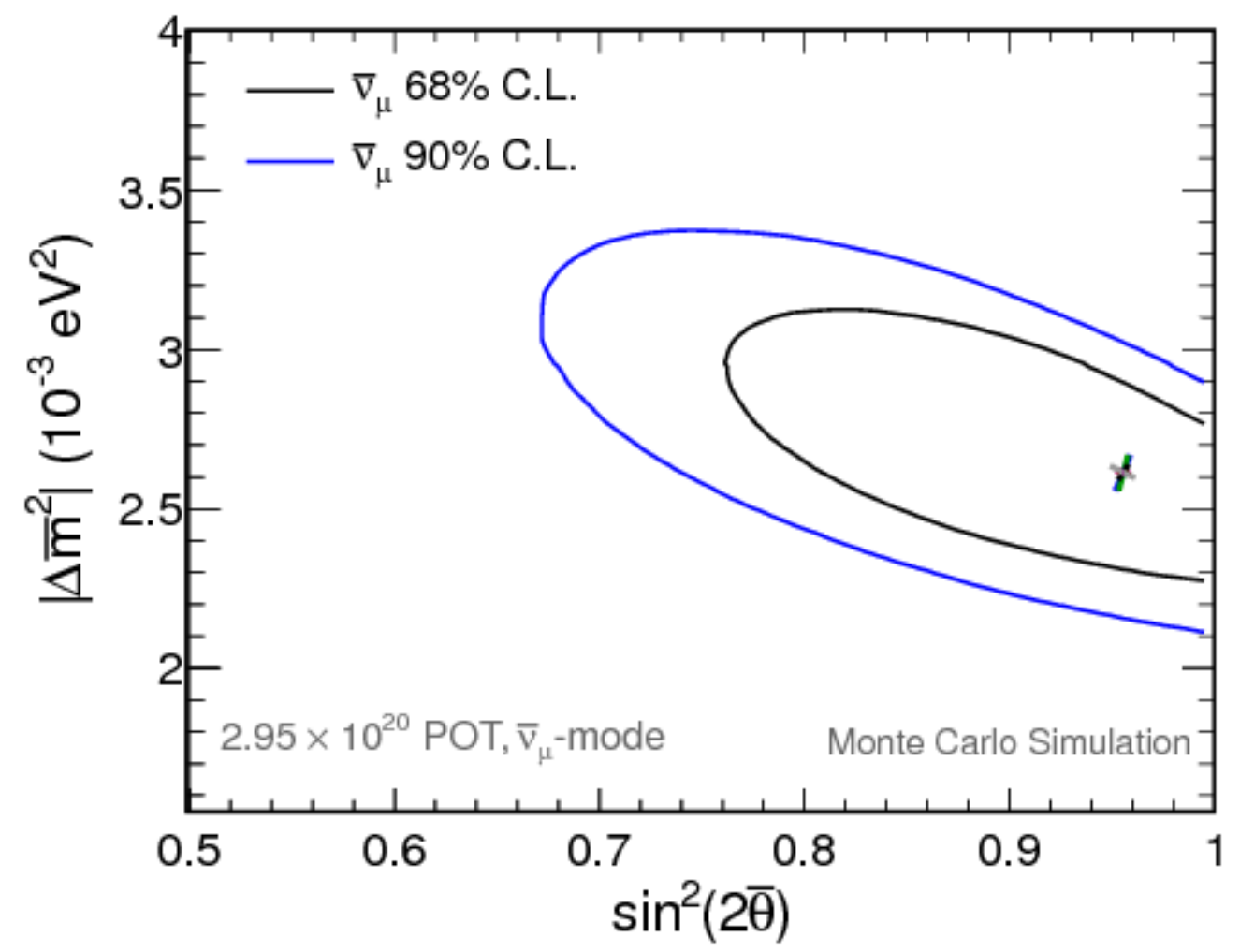

Figure 4.34: The effect of systematics shown in Fig. 4.32 overlaid with the statistical sensitivity. 


\begin{tabular}{|l|c|c|c|c|c|}
\hline Systematic & Shift & $\Delta \bar{m}^{2}\left(\times 10^{-3} \mathrm{eV}^{2}\right)$ & $\sin ^{2}(2 \bar{\theta})$ & $\delta\left(\Delta \bar{m}^{2}\right)\left(\times 10^{-3} \mathrm{eV}^{2}\right)$ & $\delta\left(\sin ^{2}(2 \bar{\theta})\right.$ \\
\hline \hline Nominal & - & 2.613 & 0.9558 & - & - \\
\hline Normalization & $+1.6 \%$ & 2.589 & 0.9532 & -0.02370 & -0.002529 \\
\hline Muon Energy & $-1.6 \%$ & 2.637 & .9582 & 0.02399 & 0.002413 \\
\hline Absolute Shower & $+1 \sigma$ & 2.669 & .9579 & 0.05585 & 0.002128 \\
Energy & $-1 \sigma$ & 2.556 & .9536 & -0.05656 & -0.002182 \\
\hline ND Relative & $-1 \sigma$ & 2.668 & .9591 & 0.05527 & 0.003374 \\
Shower Energy & $+1.9 \%$ & 2.621 & .9518 & 0.008490 & -0.004006 \\
\hline FD Relative & $-1.9 \%$ & 2.605 & .9596 & -0.008150 & 0.003845 \\
Shower Energy & $+1.1 \%$ & 2.616 & .9584 & 0.002870 & 0.002596 \\
\hline Neutral-current & $-1.1 \%$ & 2.609 & .9534 & -0.003770 & -0.002373 \\
Background & $+20 \%$ & 2.625 & .9492 & 0.01204 & -0.006614 \\
\hline Wrong-sign & $-20 \%$ & 2.601 & .9626 & -0.01230 & 0.006834 \\
Background & $+30 \%$ & 2.614 & .9560 & 0.0006400 & 0.0002100 \\
\hline Flux & $-30 \%$ & 2.6123 & .9555 & -0.0005230 & -0.0002460 \\
\hline Acceptance & $+1 \sigma$ & 2.617 & .9558 & 0.004460 & .00007600 \\
& $-1 \sigma$ & 2.608 & .9557 & -0.004820 & -0.00009900 \\
\hline Sum of & $+1 \sigma$ & 2.618 & .9546 & 0.00552 & -0.001168 \\
Cross-sections & $-1 \sigma$ & 2.607 & .9569 & -0.00541 & 0.001139 \\
\hline
\end{tabular}

Table 4.2: Systematic uncertainties and the resulting shift in the best fit values of $\Delta \bar{m}^{2}$ and $\sin ^{2} 2 \bar{\theta}$ from the nominal case. Cross section systematics are added in quadrature. 


\subsection{NON-STANDARD INTERACTIONS ANALYSIS}

To search for non-standard interactions a combined fit is performed to the antineutrino dataset of Chapter 4 and a neutrino dataset collected in forward horn current mode. Many of the features such as selection variables and systematic uncertainties are similar to the antineutrino oscillation analysis; the differences are detailed below along with the fitting procedure.

\subsection{THE NEUTRINO DATASET}

MINOS collected data in neutrino-mode for four years before switching to antineutrinomode. The neutrino dataset used to measure non-standard interactions comprises three low energy run periods for a total exposure of $7.09 \times 10^{20}$ protons on target. Neutrino oscillation parameters have been measured [105] by analyzing this data ${ }^{1}$ in a nearly identical manner to the antineutrino oscillation analysis presented above. One important difference is that the neutrino oscillation analysis does not require a charge sign selection. This is justified for the neutrino oscillation analysis because neutrino-mode data has a very small antineutrino component; a cut to remove this results in a loss of efficiency and sensitivity. The published results from this MINOS measurement are

$$
\left|\Delta m_{\mathrm{atm}}^{2}\right|=2.32_{-0.08}^{+0.12} \times 10^{-3} \mathrm{eV}^{2} \text { and } \sin ^{2}\left(2 \theta_{23}\right)>0.90 \quad(90 \% \text { C.L }) .
$$

which is the most precise measurement (5\%) of the atmospheric mass splitting. Confidence

\footnotetext{
${ }^{1}$ The neutrino oscillation analysis includes an additional set of high energy mode data for a total of $7.2 \times 10^{20}$ POT exposure.
} 
intervals are shown in Fig. 5.1 overlaid with results from the Super Kamiokande experiment. Detailed accounts of the steps of the neutrino oscillation analysis are presented in $[79,106$, 107, 108, 109].

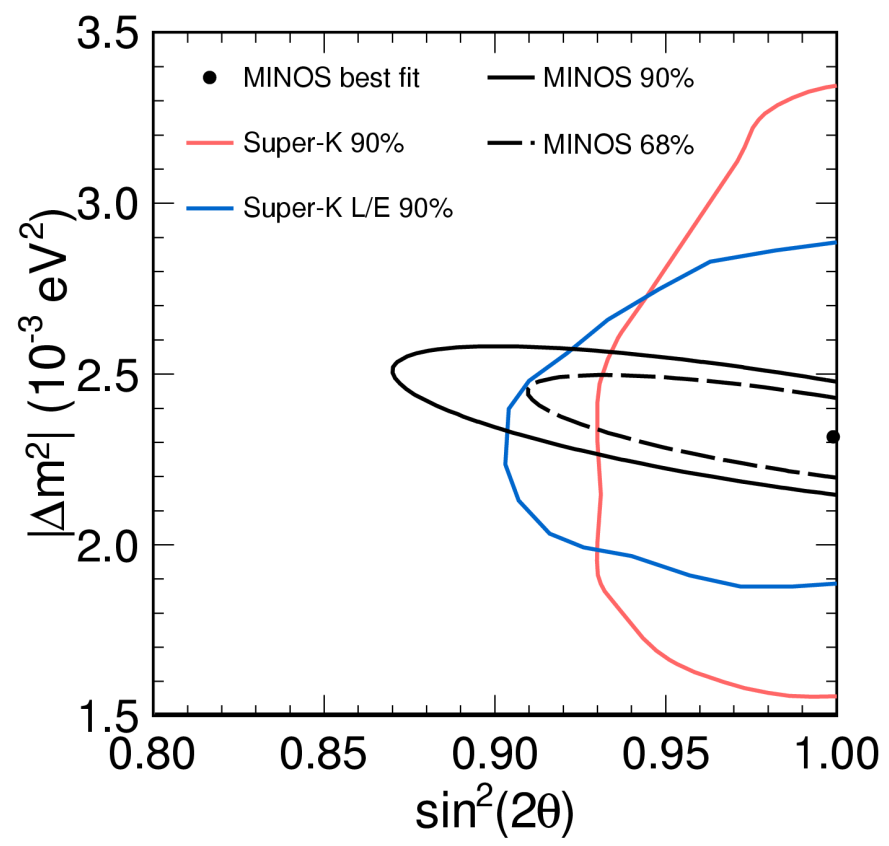

Figure 5.1: Charged-current neutrino oscillation contours are shown in black. Blue and red curves show contours from Super-K [54].

\subsubsection{Separation of charged-current and neutral-current events}

In addition to the $k \mathrm{NN}$ separator used in the antineutrino oscillation analysis, the neutrino selection employs a second $\mathrm{CC} / \mathrm{NC}$ separation method to increase efficiency in the low energy region. This second separator also uses a $k \mathrm{NN}$ algorithm tailored uniquely to the needs of short tracks, with the purpose of increasing the acceptance of short charged current tracks without introducing a large neutral current background. The $k \mathrm{NN}$ space is constructed by three variables. 
- Number of scintillator planes in track. This variable is identical to the number-ofplanes variable of the primary $k \mathrm{NN}$.

- Energy deposited at the end of the track. This variable measures the total pulse height of the last five planes of the track. It is complementary to the mean pulse height variable used by the primary $k \mathrm{NN}$ but aims to capture increases in the pulse height at the end of the track. A sharp increase in energy deposition at the end of the track may indicate that the particle is a high energy pion or proton being captured.

- U and V view scattering variables. The scattering variables quantify the amount of scattering in one of transverse vs. longitudinal ( $\mathrm{U}$ vs. Z or V vs. Z) views. At low energy, distinguishing neutral and charged current tracks is a challenge because a short track is easy to lose in the shower. This variable can distinguish straight short tracks from those with scatter which improves the separation of low energy muon tracks from showers.

The resulting $\mathrm{CC} / \mathrm{NC}$ discriminator distribution, along with the primary discriminator is shown in Fig. 5.2.
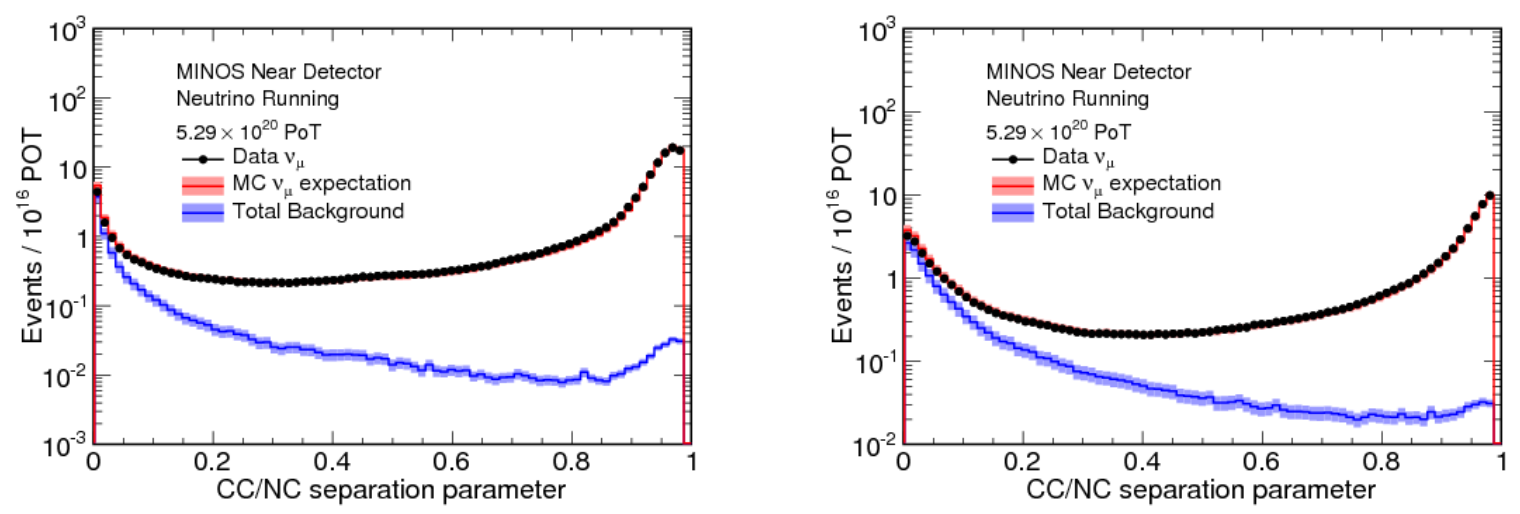

Figure 5.2: The primary $\mathrm{CC} / \mathrm{NC}$ separation variable (left) and the additional low energy separator(right) used to identify muon tracks in the neutrino sample, shown before the selection is applied. The red histogram shows the MC prediction with the systematic error band, blue shows the background and black dots denote Near Detector data. 


\subsubsection{Near Detector Sample}

The charged-current selection is a logical $\mathrm{OR}$ of the two $k \mathrm{NN}$ discriminators where events with $k \mathrm{NN}_{\mathrm{ID}}>0.25$ or $k \mathrm{NN}_{\mathrm{ID}}^{\text {new }}>0.5$ are kept. CC neutrino interactions have a $\mu^{-}$in the final state therefore the charge sign requirement is $q / p<0$. The track end point requirement to cut out the coil hole region is not held for this analysis. The efficiency and contamination of the overall neutrino selection is shown in Fig. 5.3 at the Near Detector.

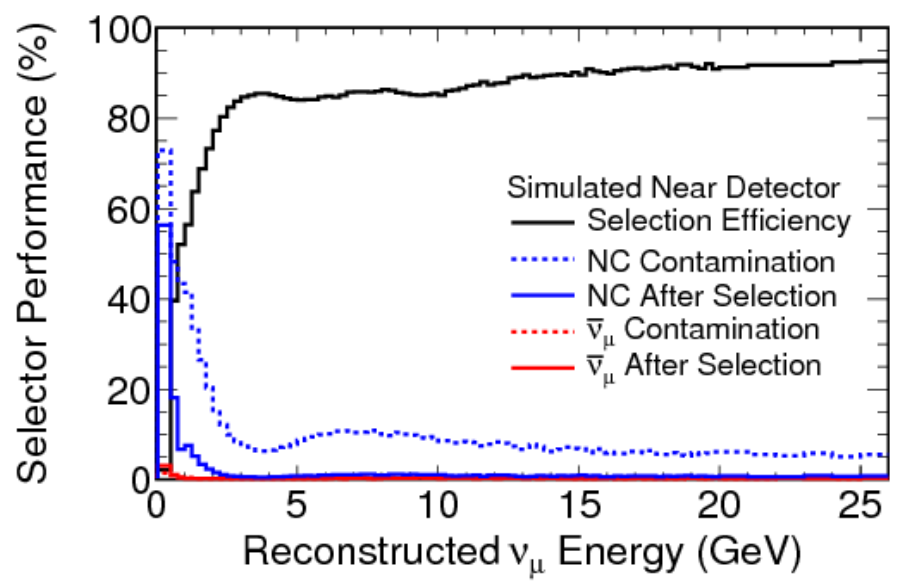

Figure 5.3: The black line shows the efficiency of the neutrino selector, dashed lines show the antineutrino (red) and NC (blue) contamination before selection, solid colored lines show contamination after selection.

Fig. 5.4 shows the neutrino energy, track energy, shower energy and inelasticity distributions. The red band shows the Monte Carlo with a systematic error band calculated by adding the effect of each systematic uncertainty in quadrature; details of systematic uncertainties are given in Section 4.8 and 5.4. The band includes the effects of the uncertainty in the beam flux and neutrino cross sections, which mostly cancel between the two detectors and have a negligible impact on the results. The blue band shows the size of the total background consisting of mis-identified tracks of opposite sign and neutral-current events. The data shows good agreement with Monte Carlo and the disagreements are within limits of the systematic error. 

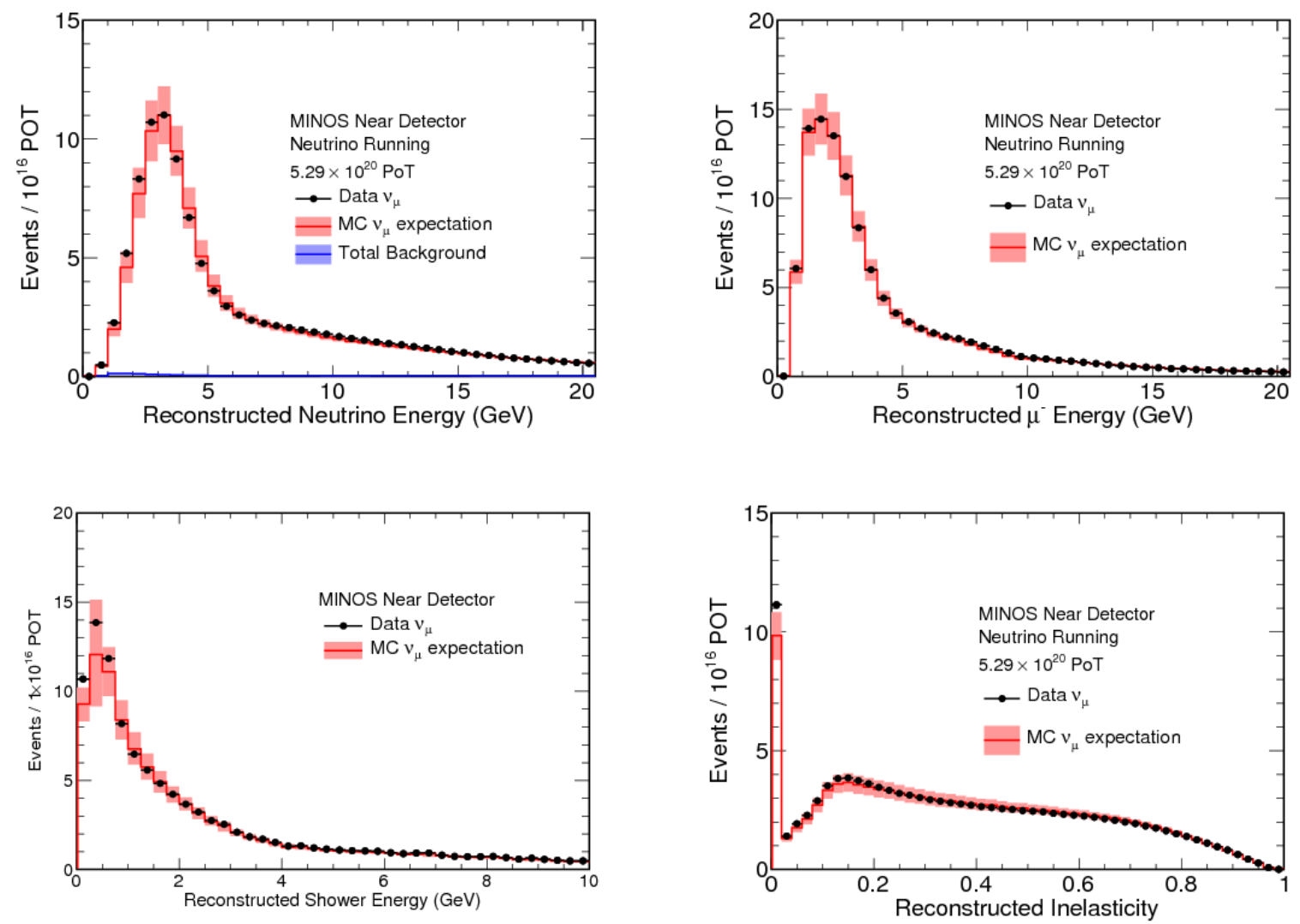

Figure 5.4: Neutrino energy (top left), track energy (top right), shower energy (bottom left) and inelasticity distributions at the Near Detector for the neutrino sample. Black dots represent ND data, red curve represents Monte Carlo where the pink band is the systematic error. The blue band shows the total background of neutral current and wrong-sign events. 
Fig. 5.5 shows the squared radius and longitudinal distributions of track vertices. Many other distributions including distributions of track shapes and sizes, kinematic variables, angular distributions and timing were investigated; Monte Carlo models data well.
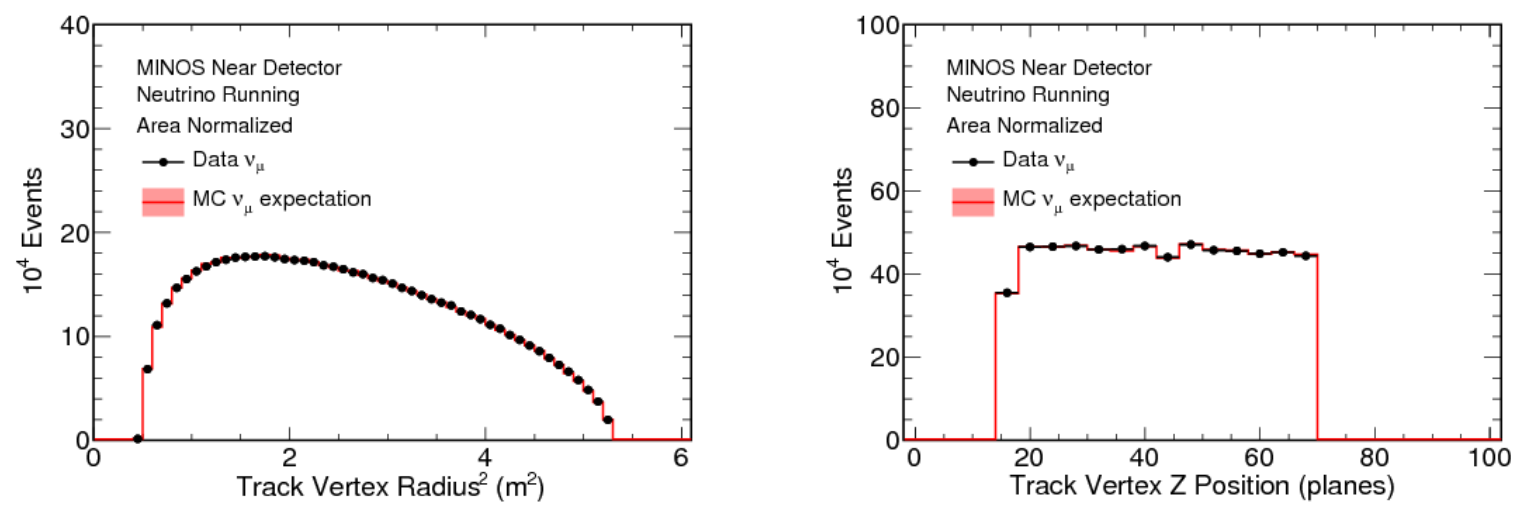

Figure 5.5: The squared track vertex (left) position in meters and the longitudinal track vertex position in units of number of planes. Black dots represent ND data and the red curve represents Monte Carlo. 


\subsubsection{Far Detector Sample}

The Far Detector selection is the same as the Near with the additional requirement that the cosine of the angle between the track direction and beam axis is smaller than 0.6. The efficiency and contamination of the overall neutrino selection is shown in Fig. 5.6 at the Far Detector.

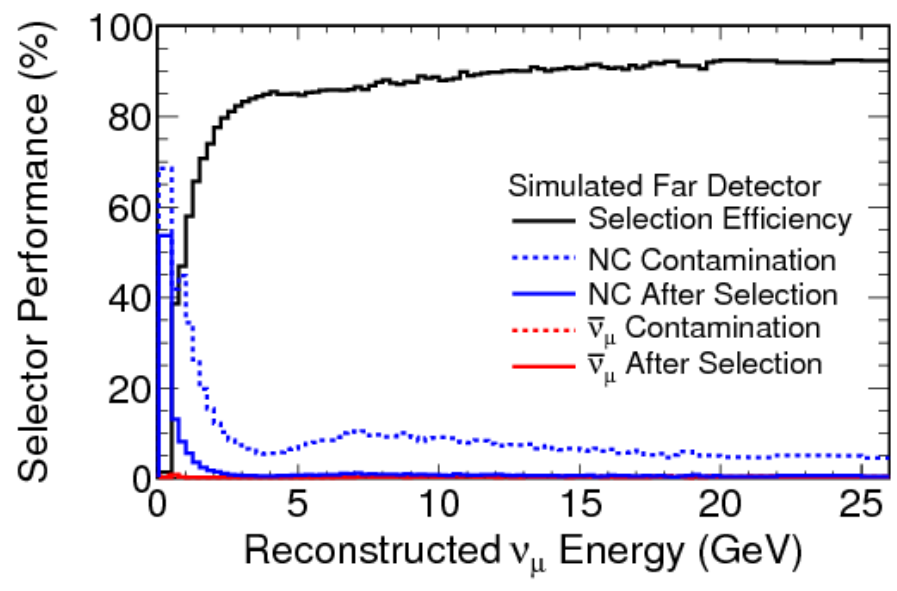

Figure 5.6: The black line shows the efficiency of the neutrino selector, dashed lines show the antineutrino (red) and neutral current (blue) contamination before selection, solid colored lines show contamination after selection at the Far Detector.

With $7.09 \times 10^{20}$ POT of neutrino data, a total of 2073 events are expected in the case of no oscillations; we observe 1654 events. Track vertex and end distributions are shown in Fig. 5.7. Track vertices have a flat squared radius distribution as expected. Tracks curve towards the coil hole. The track and shower energy distributions can be seen in Fig. 5.8 and present good agreement between data and simulation. The distribution of the angle that tracks make with the beam axis is shown in Fig. 5.9. Error bars are Poisson for bins with fewer than 50 events as prescribed by [101]. 

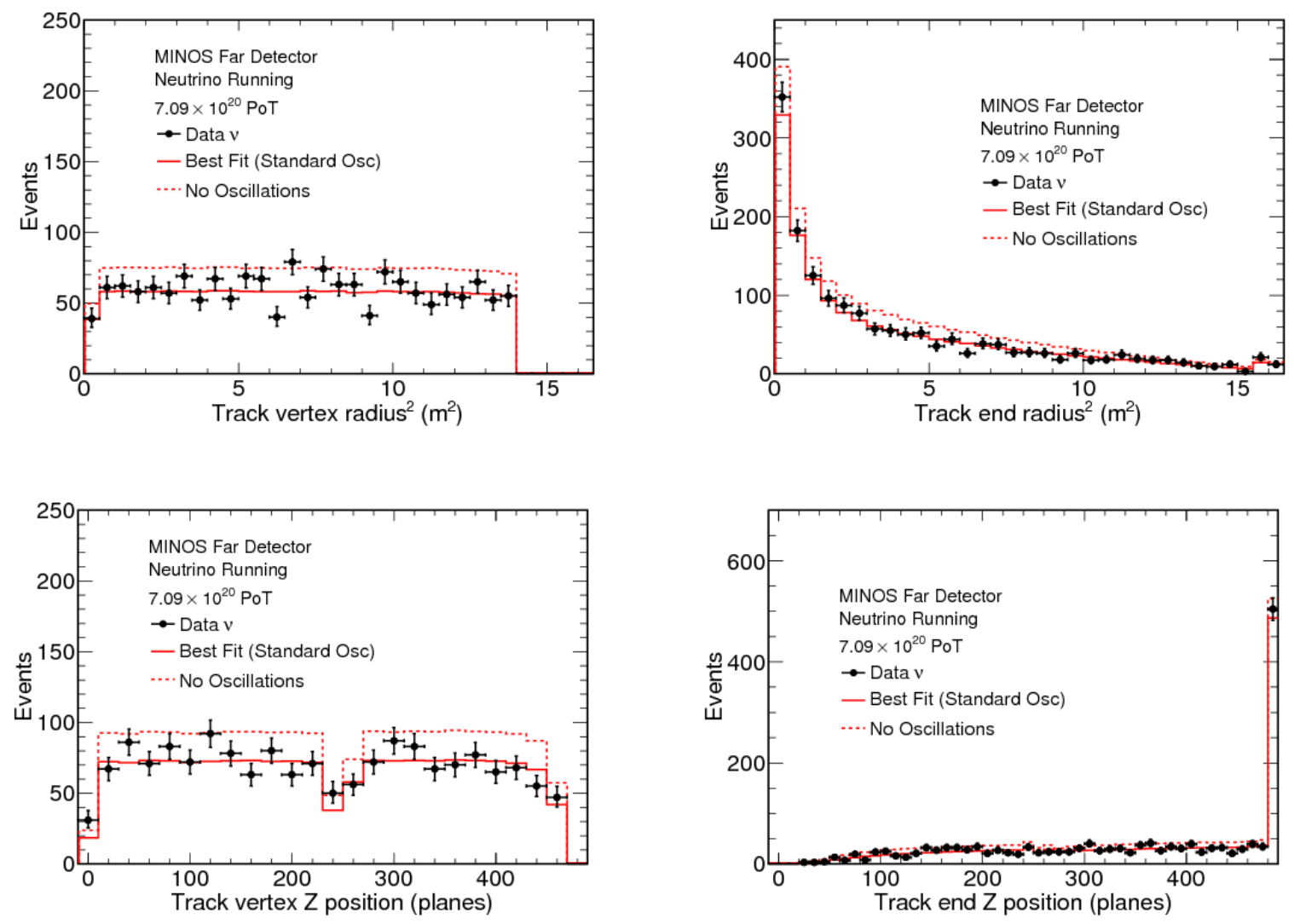

Figure 5.7: Track vertex and end squared radius distributions of the neutrino sample at the Far Detector. The dashed red curve is the Monte Carlo simulation with no oscillations, the solid red curve is best vacuum oscillation fit and black dots are data. 

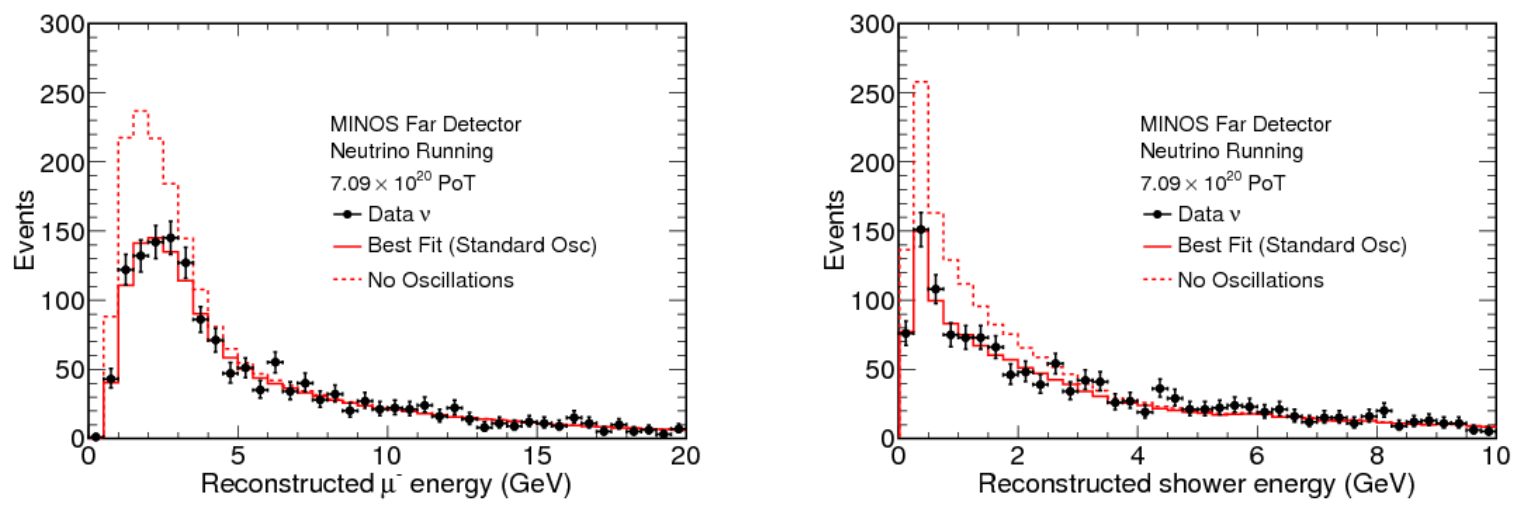

Figure 5.8: Muon and shower energy distributions for the neutrino sample in the Far Detector. The dashed red curve is the Monte Carlo simulation with no oscillations, the solid red curve is best vacuum oscillation fit and black dots are data.

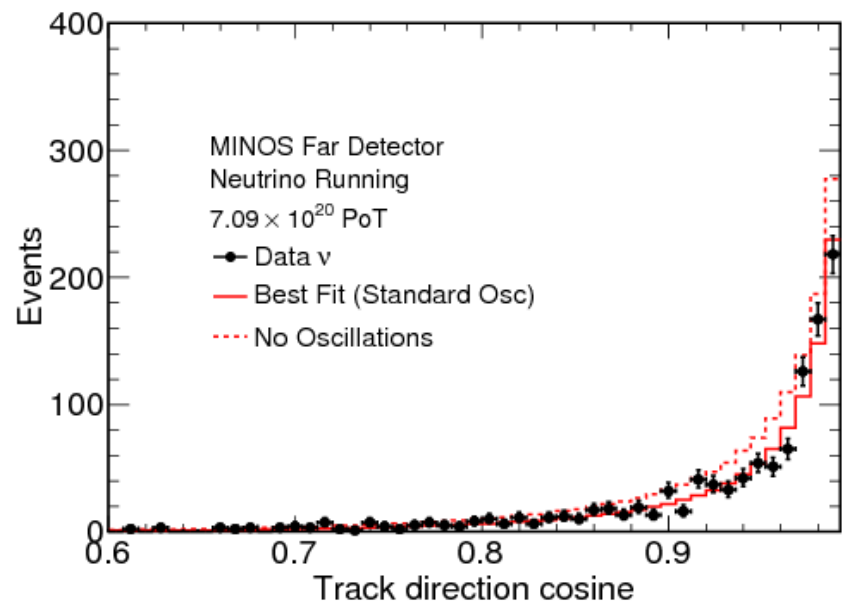

Figure 5.9: Distribution of the cosine of the angle between tracks and the beam axis. Events with $\cos \theta<0.6$ are excluded. Black dots represent FD data, the solid red curve represents Monte Carlo with best fit vacuum oscillations and dashed curve is without oscillations. 


\subsection{ANTINEUTRINO SELECTED SAMPLE}

The Near Detector antineutrino dataset is selected in a nearly identical way to the antineutrino oscillation analysis with the exception of the track end cut to remove events ending in the coil hole (see Section 4.4 and Fig. 4.8 for this cut). The Far Detector selection is identical. The ND efficiency and contamination of the antineutrino selection is shown in Fig. 5.10. The efficiency is seen to be larger than the version for antineutrino oscillations in the previous chapter because the track end cut excluding the coil hole region is not applied to keep the neutrino and antineutrino samples more similar.

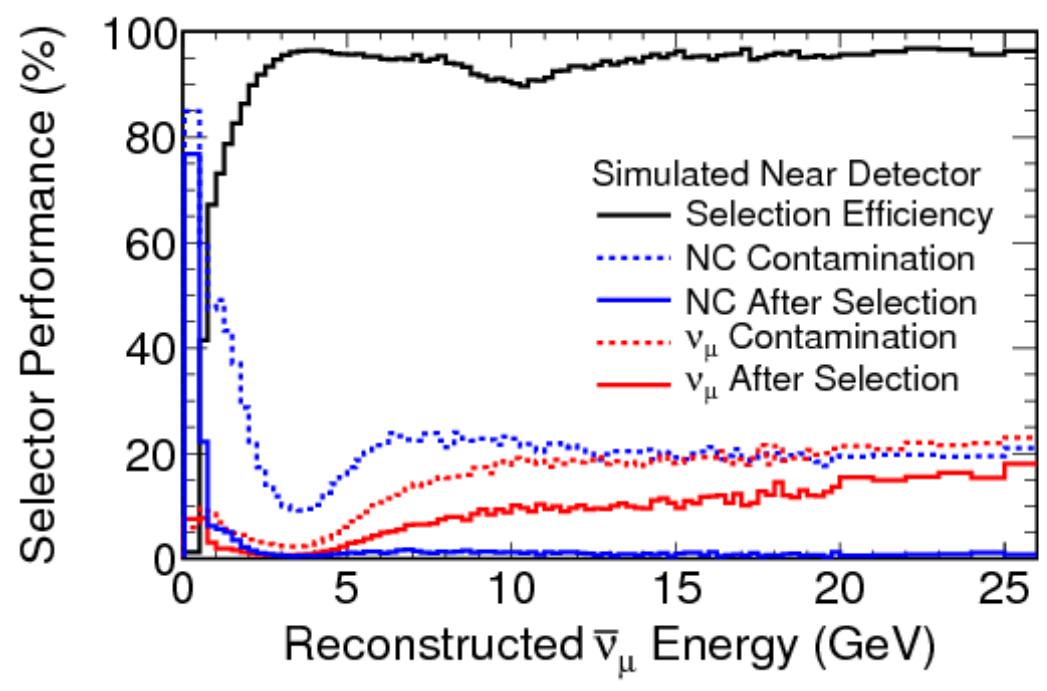

Figure 5.10: The black line shows the efficiency of the antineutrino selector, dashed lines show the neutrino (red) and NC (blue) contamination before selection, solid colored lines show contamination after selection at the Near Detector. 


\subsection{NEAR TO FAR EXTRAPOLATION}

The extrapolation procedure is explained in detail in Section 4.7. This analysis uses the same extrapolation method. The measured Near Detector data is extrapolated by using the Beam Matrix method to obtain a Far Detector prediction. Neutrinos and antineutrinos are extrapolated separately to take into account the differences in the background, but neutrino and antineutrino mass-splittings and mixing angles are always taken to be identical. Within the neutrino sample, each run is extrapolated separately to account for flux differences due to target decay and for the presence of Helium in the decay pipe in Run III.

\subsection{SYSTEMATIC UNCERTAINTIES}

The set of uncertainties that can affect disappearance measurements are given in Section 4.8 in detail. The same set of uncertainties applies to this measurement, with two exceptions. The acceptance systematic is not taken into consideration in this analysis. Also, the wrong sign background uncertainty has different magnitudes for the neutrino and antineutrino samples.

The wrong-sign background uncertainty of the antineutrino sample is described in Section 4.8.4. The antineutrino background uncertainty in the neutrino sample is evaluated similarly to be $70 \%$. The antineutrino component in neutrino-mode is much smaller, so although there is a larger percentage uncertainty, its effect on the result is still small (indeed smaller than the wrong-sign uncertainty in the antineutrino sample).

The neutral current background uncertainty in the neutrino sample is $20 \%$ (see Section 4.8.4). In the neutrino sample, a data-driven method is used to evaluate the size of

this uncertainty [110] and finds the total uncertainty to be $19 \%$, therefore an overall $20 \%$ uncertainty is taken in both neutrino and antineutrino datasets. 


\subsection{FITTING THE DATA}

The neutrino and antineutrino data is fit simultaneously to the non-standard interactions model where the disappearance probability is given by Eq. (1.85). The best fit to data is calculated by minimizing the log-likelihood function [18] between data and predictions generated at a range of $\Delta m^{2}, \sin ^{2}(2 \theta), \epsilon$ sets. The fit is performed in 100 bins, $i$, and five run periods, $r$. The value of the mixing angle is constrained to be physical, that is, the allowed range is $0<\sin ^{2}(2 \theta)<1$.

Systematic uncertainties are taken into consideration in the fit. As shown in section 5.4, the overall effect of systematics is much smaller than the statistical uncertainty. However, the relative statistical size of the neutrino and antineutrino samples is very different (about 1600 neutrino events and 200 antineutrino). The inclusion of the effect of systematics on the analysis ensures that the neutrino sample does not "pull" the fit disproportionately. Systematic uncertainties are included in the fit as penalty terms, meaning the fitter is allowed to vary each systematic uncertainty term (within user-defined limits) to minimize the likelihood at every grid point. The final likelihood function with systematics is given by

$$
-2 \ln \lambda=2 \sum_{r=1}^{5} \sum_{i=1}^{N} n_{r i}^{\text {pred }}-n_{r i}^{\mathrm{data}} \ln \frac{n_{r i}^{\mathrm{data}}}{n_{r i}^{\text {pred }}}+\sum_{\alpha=1}^{4}\left(\frac{S_{\alpha}-S_{\alpha}^{\prime}}{\sigma_{\alpha}}\right)^{2}
$$

where $S_{\alpha}$ denotes a systematically shifted prediction with systematic $\alpha$, and $\sigma_{\alpha}$ are the sizes of the one-sigma level for each uncertainty.

The four largest systematic uncertainties were included in the fit: muon energy scale, hadronic energy scale, neutral current background and Near to Far normalization. The systematic error bands in Fig. 5.11 show the fractional effect of the four largest uncertainties on the Far Detector prediction in the neutrino and antineutrino samples.

From three free parameters, it is possible to construct three two-dimensional likelihood surfaces. This is performed by marginalizing over the parameter that is not being shown. To calculate a likelihood surface $y$ vs. $x$ with marginalized parameter $z$, the likelihood at a point $\left(x_{o}, y_{o}\right)$ is computed by fixing the values of $x=x_{o}$ and $y=y_{o}$ and finding the minimum of Eq. 5.2 by varying $z$. The information of the $z_{o}$ that gives the minimum likelihood is lost 

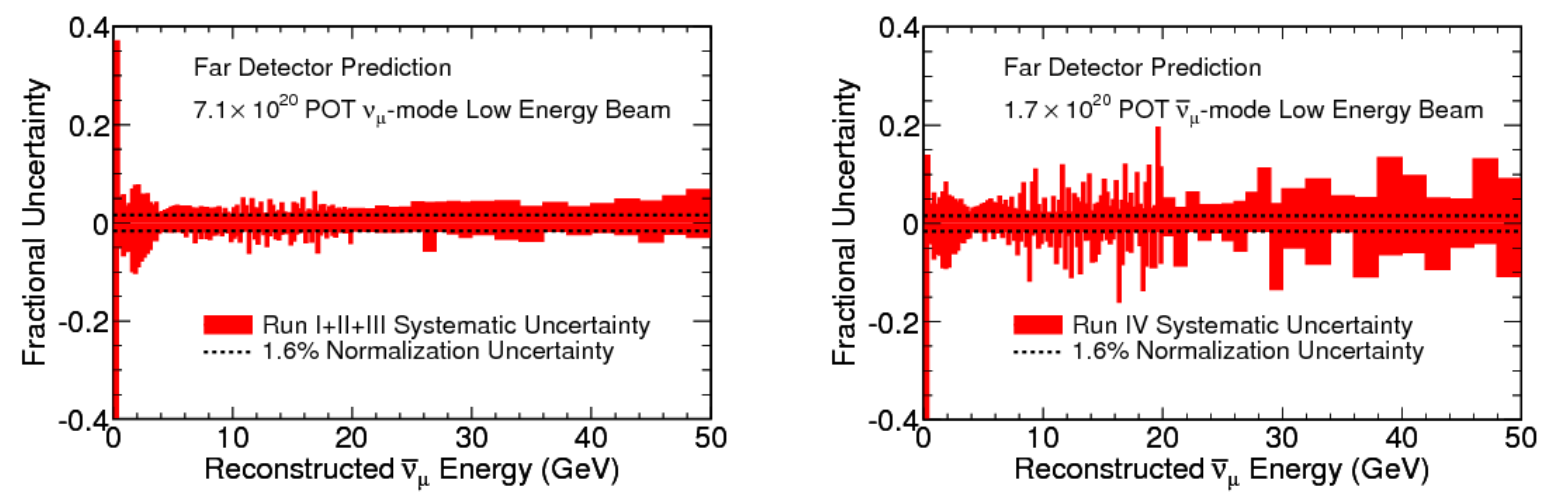

Figure 5.11: The neutrino (left) and antineutrino fractional systematic error bands from the four largest systematic uncertainties: muon energy scale, shower energy scale, NC background and normalization.

for the $x y$-surface, thus the term marginalization. This is necessary since we are visually limited to presenting contours in two dimensional surfaces; three such surfaces are obtained by the same method in all possible two-variable pairs.

The best fit to the NSI model with a common mass splitting and mixing angle yields:

$$
\begin{aligned}
\Delta m^{2} & =2.39_{-0.105}^{+0.135} \times 10^{-3} \mathrm{eV}^{2} \\
\sin ^{2}(2 \theta) & >0.91(90 \% \text { C.L. }) \\
\epsilon_{\mu \tau} & =-0.068_{-0.080}^{+0.083}
\end{aligned}
$$

Systematic pulls, i.e. the amount by which the prediction is shifted by a systematic, shown in Table 5.1 are small which indicates that the allowed range of systematic shift is sufficient and that the effect of systematics is not biasing the measurement.

Fig. 5.12 shows the neutrino prediction at the Far Detector in the case of no oscillations and at the best fit parameters overlaid with data and the neutral current background prediction. The bottom plot in the same figure shows the ratio of each spectrum to the no-oscillations case where the energy dependent deficit can be clearly seen. Fig. 5.13 shows 
the same histograms for the antineutrino sample.

\begin{tabular}{|c|c|}
\hline Systematic uncertainty & $\operatorname{Pull}(\sigma)$ \\
\hline Normalization & 0.012 \\
Track energy scale & -0.017 \\
Shower energy scale & -0.17 \\
NC background & -0.0091 \\
\hline
\end{tabular}

Table 5.1: Four systematic uncertainties included in the fit and the amount by which the prediction needs to be shifted by each ("pulls") to obtain the best fit. 

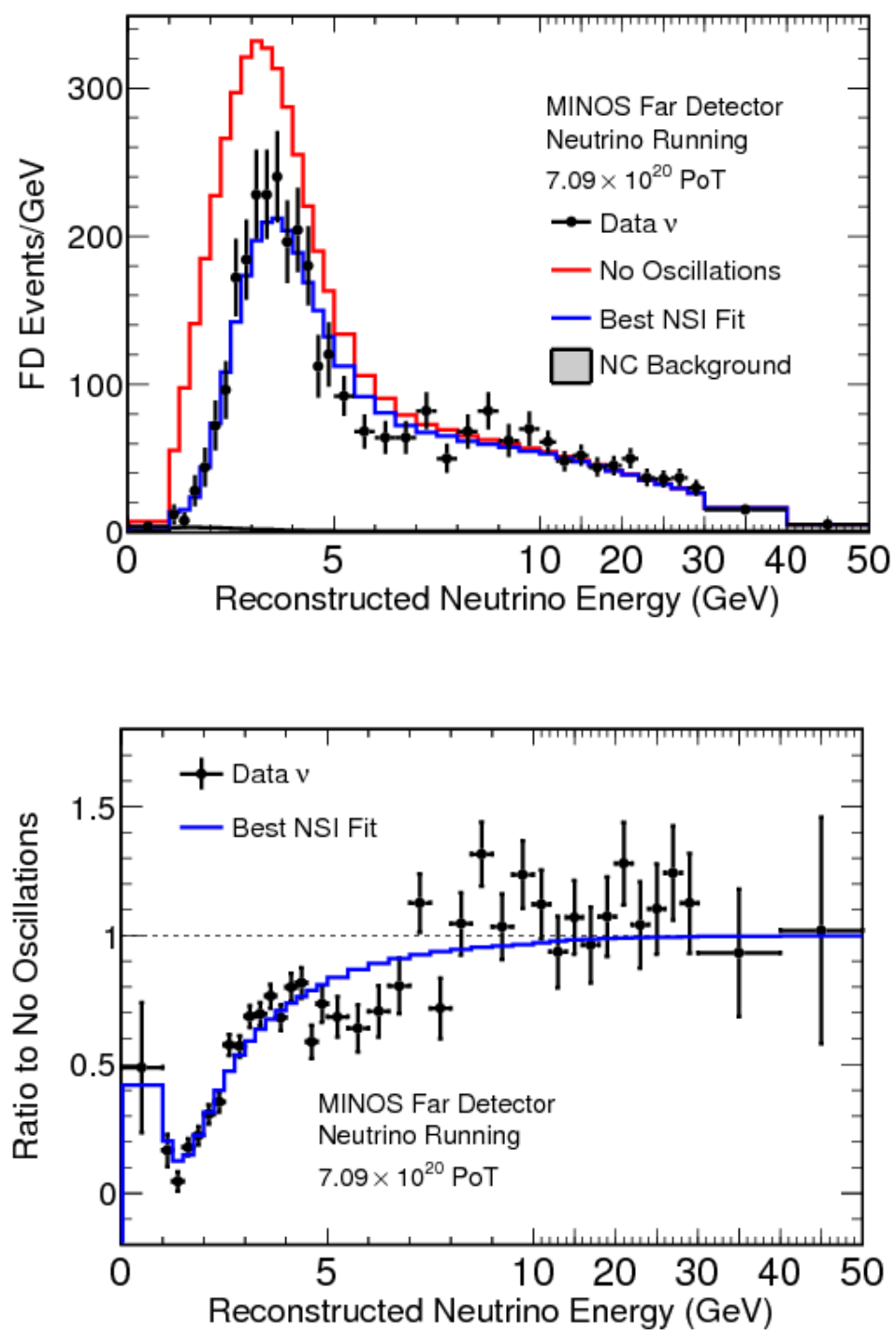

Figure 5.12: Top: Far Detector neutrino prediction in the case of no oscillations in red and at the NSI best fit parameters in blue. Black dots represent data with Poisson error bars. The gray solid histogram shows the neutral current background. Bottom: The ratio of data and best fit to null oscillations. 

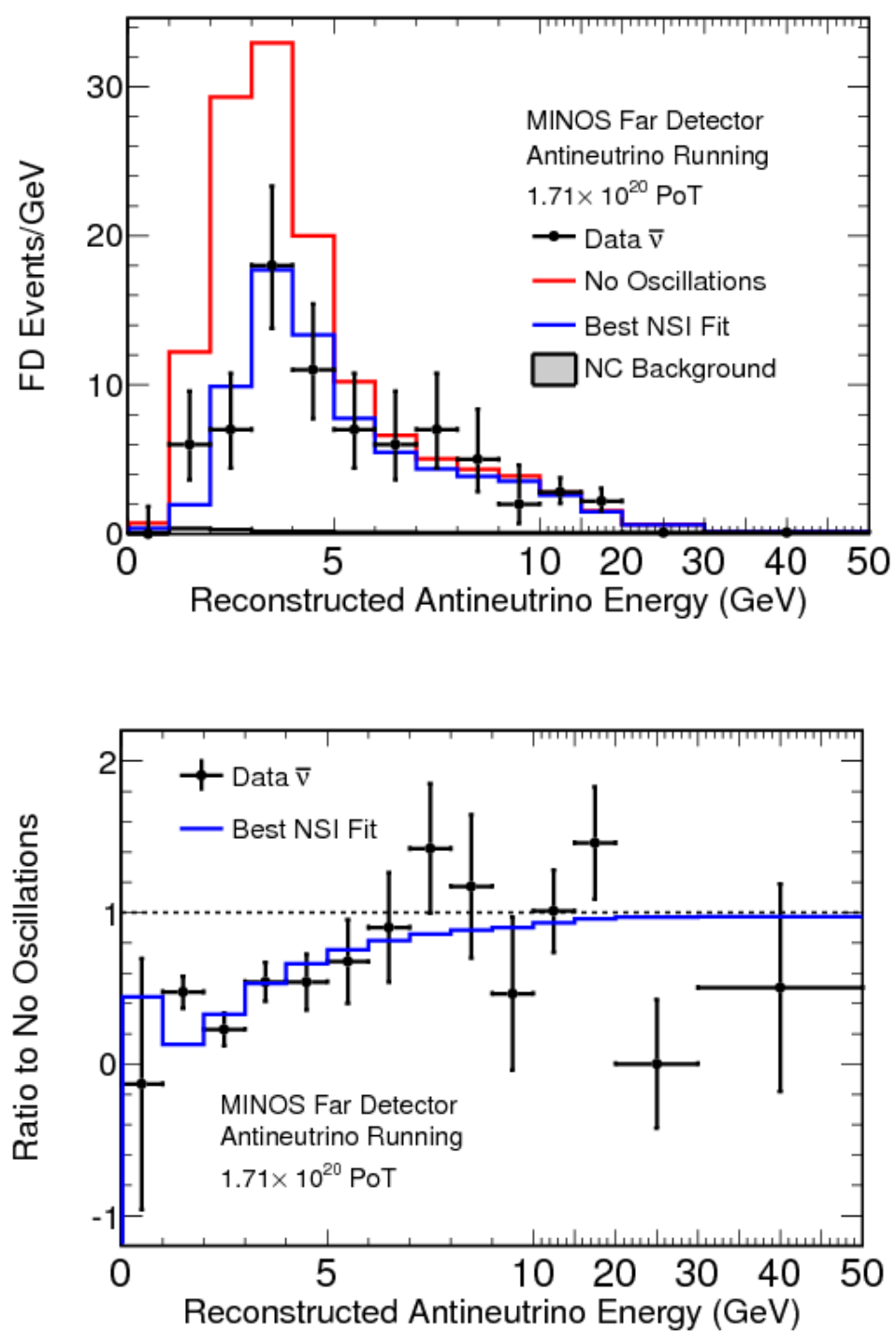

Figure 5.13: Top: Far Detector antineutrino prediction in the case of no oscillations in red and at the NSI best fit parameters in blue. Black dots represent data with Poisson error bars. The gray solid histogram shows the neutral current background. Bottom: The ratio of data and best fit to null oscillations. 
The $\chi^{2}$ per degree of freedom of the fit (in 500 bins with 7 free parameters) is 518/492. The allowed regions are shown in Fig. 5.14 while Fig. 5.15 shows the one-dimensional allowed region for each parameter. In these plots, the unshown two variables are marginalized as described above. The $1 \sigma$ errors on the best fit values quoted above come from these $\Delta \chi^{2}$ distributions.
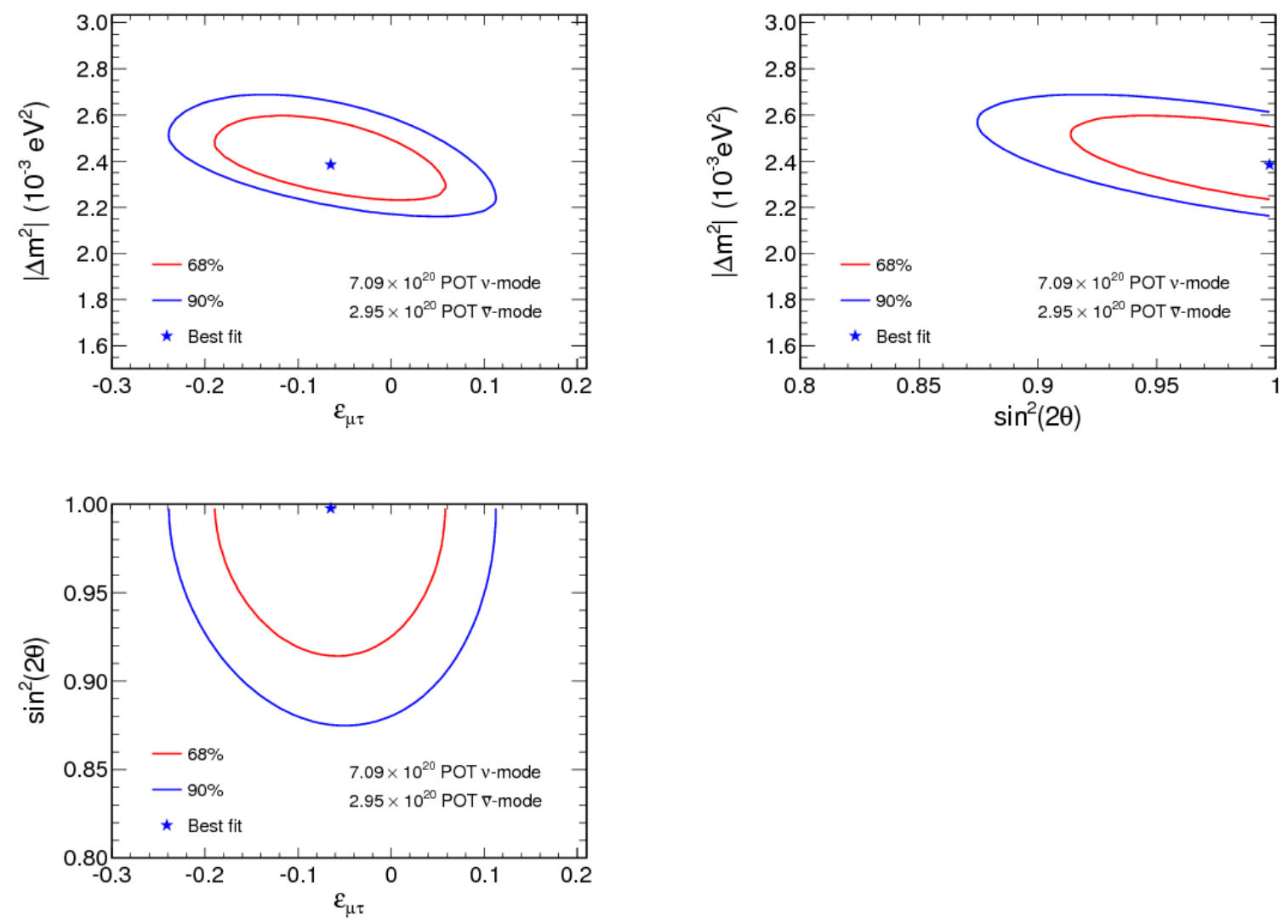

Figure 5.14: The $68 \%$ and $90 \%$ contours include the four largest systematics. 

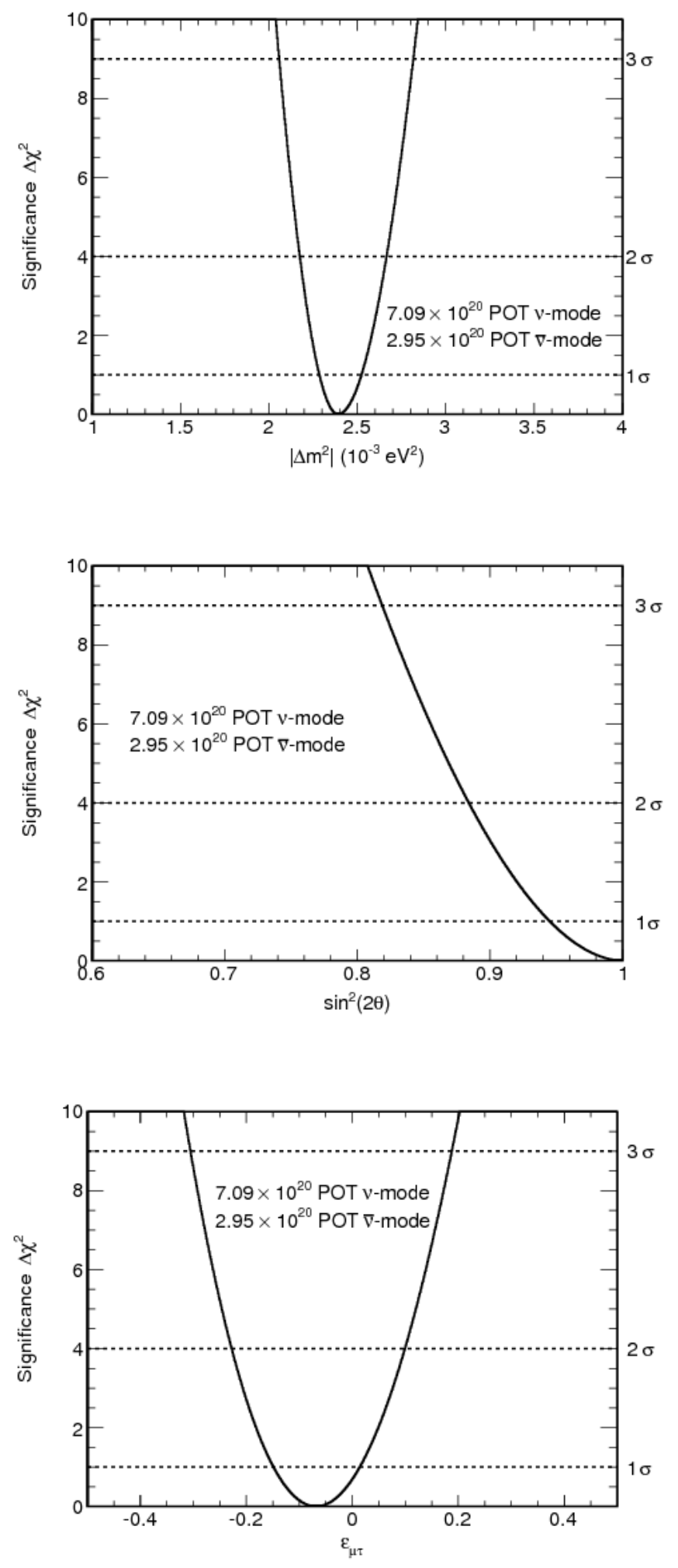

Figure 5.15: $\Delta \chi^{2}$ for one dimensional fits where all other parameters are marginalized. Dashed lines show 1, 2 and $3 \sigma$ coverage. 


\subsubsection{Evaluation of NSI parameter Uncertainties}

The effect of systematic uncertainties are calculated by generating five sets of systematically shifted fake data corresponding to the three neutrino and two antineutrino run periods with each systematic uncertainty, as well as an unshifted set (nominal). Runs I, II and III are neutrino-mode runs and are produced with the same size systematic, always correlated with each other. This makes up the shifted neutrino dataset. Shifted Run IV and Run VII fake data comprise the antineutrino dataset. Combined fits to the shifted neutrino and antineutrino sets are performed and the shifted best fits are compared to the nominal best fit point. The difference between nominal and shifted best fit values of each parameter gives the effect of a given systematic on that parameter. The total systematic uncertainty is computed by adding the differences in quadrature.

Correlation of each systematic uncertainty between the neutrino and antineutrino samples is taken into consideration. The wrong sign background systematic is by definition fully correlated between the two samples. In order to account for partial correlations of other systematics, the effect of the uncertainty has been evaluated twice, once assuming full correlation and once full uncorrelation. The resulting deviations from the nominal best fit point in the correlated and uncorrelated evaluations are small and do not affect the overall size of the systematic uncertainty significantly. Moreover, the analysis is limited by statistics and the neutrino-antineutrino correlations of systematics have no effect on the extrapolation or fit results. With this verification, uncertainties are assumed to be fully correlated between neutrino and antineutrino samples since this makes the computationally simplest fitter.

Table 5.2 shows the nominal and shifted best fit points and the sizes of deviations from nominal with each systematic. These deviations can be seen graphically in Fig. 5.16. Individual fits to these shifted sets of data are performed to evaluate the relative size of the effect of each systematic and to graphically compare to the size of the statistical uncertainty. The incorporation in the fitting mechanism is separate as described above in Sec. 5.5. 


\begin{tabular}{|c|c|c|c|c|c|c|c|}
\hline Systematic & Shift & $\Delta m^{2}$ & $\sin ^{2} \theta$ & $\epsilon_{\mu \tau}$ & $\delta\left(\Delta m^{2}\right)$ & $\delta\left(\sin ^{2} \theta\right)$ & $\delta\left(\epsilon_{\mu \tau}\right)$ \\
\hline \hline Nominal & - & 2.6482 & 1.0045 & 0.2674 & 0.0000 & 0.0000 & 0.0000 \\
\hline \multirow{2}{*}{ Normalization } & $+1.6 \%$ & 2.6305 & 1.0025 & 0.2769 & -0.0176 & -0.0020 & 0.0095 \\
& $-1.6 \%$ & 2.6657 & 1.0064 & 0.2574 & 0.0175 & 0.0019 & -0.0100 \\
\hline \multirow{2}{*}{ NC Background } & $+20 \%$ & 2.6582 & 0.9975 & 0.2687 & 0.0101 & -0.0070 & 0.0013 \\
& $-20 \%$ & 2.6379 & 1.0117 & 0.2662 & -0.0103 & 0.0072 & -0.0013 \\
\hline \multirow{2}{*}{ Muon Energy } & $+1 \sigma$ & 2.6903 & 1.0062 & 0.2687 & 0.0422 & 0.0017 & 0.0012 \\
& $-1 \sigma$ & 2.6060 & 1.0025 & 0.2663 & -0.0422 & -0.0020 & -0.0011 \\
\hline \multirow{2}{*}{ SKZP } & $+1 \sigma$ & 2.6537 & 1.0044 & 0.2653 & 0.0056 & -0.0001 & -0.0022 \\
& $-1 \sigma$ & 2.6420 & 1.0047 & 0.2698 & -0.0061 & 0.0002 & 0.0024 \\
\hline \multirow{2}{*}{ Abs ShwEn } & $+1 \sigma$ & 2.6950 & 1.0092 & 0.2598 & 0.0469 & 0.0047 & -0.0077 \\
& $-1 \sigma$ & 2.6008 & 0.9986 & 0.2762 & -0.0473 & -0.0060 & 0.0087 \\
\hline \multirow{2}{*}{ Rel ShwEn N } & $+1.9 \%$ & 2.6499 & 1.0012 & 0.2702 & 0.0018 & -0.0033 & 0.0027 \\
& $-1.9 \%$ & 2.6463 & 1.0078 & 0.2648 & -0.0019 & 0.0033 & -0.0027 \\
\hline \multirow{2}{*}{ Rel ShwEn F } & $+1.1 \%$ & 2.6426 & 1.0020 & 0.2703 & -0.0055 & -0.0025 & 0.0028 \\
& $-1.1 \%$ & 2.6535 & 1.0071 & 0.2648 & 0.0053 & 0.0025 & -0.0027 \\
\hline \multirow{2}{*}{ WS Bckg } & $+70 / 30 \%$ & 2.6488 & 1.0044 & 0.2679 & 0.0007 & -0.0001 & 0.0005 \\
& $-70 / 30 \%$ & 2.6475 & 1.0047 & 0.2670 & -0.0007 & 0.0001 & -0.0005 \\
\hline \hline \multirow{2}{*}{ Total Cross Section } & + & - & - & - & 0.0118 & 0.0045 & 0.0039 \\
& - & - & - & - & -0.0118 & -0.0045 & -0.0041 \\
\hline \multirow{2}{*}{ Total Uncertainty } & + & - & - & - & 0.0677 & 0.0108 & 0.0143 \\
& - & - & - & - & -0.0682 & -0.0114 & -0.0141 \\
\hline
\end{tabular}

Table 5.2: Systematic uncertainties and the resulting shift in the best fit values of $\Delta m^{2}$, $\epsilon$ and $\sin ^{2}(2 \theta)$ from the nominal case assuming all systematics are fully correlated between neutrino and antineutrino samples. Cross section systematics are added in quadrature. 

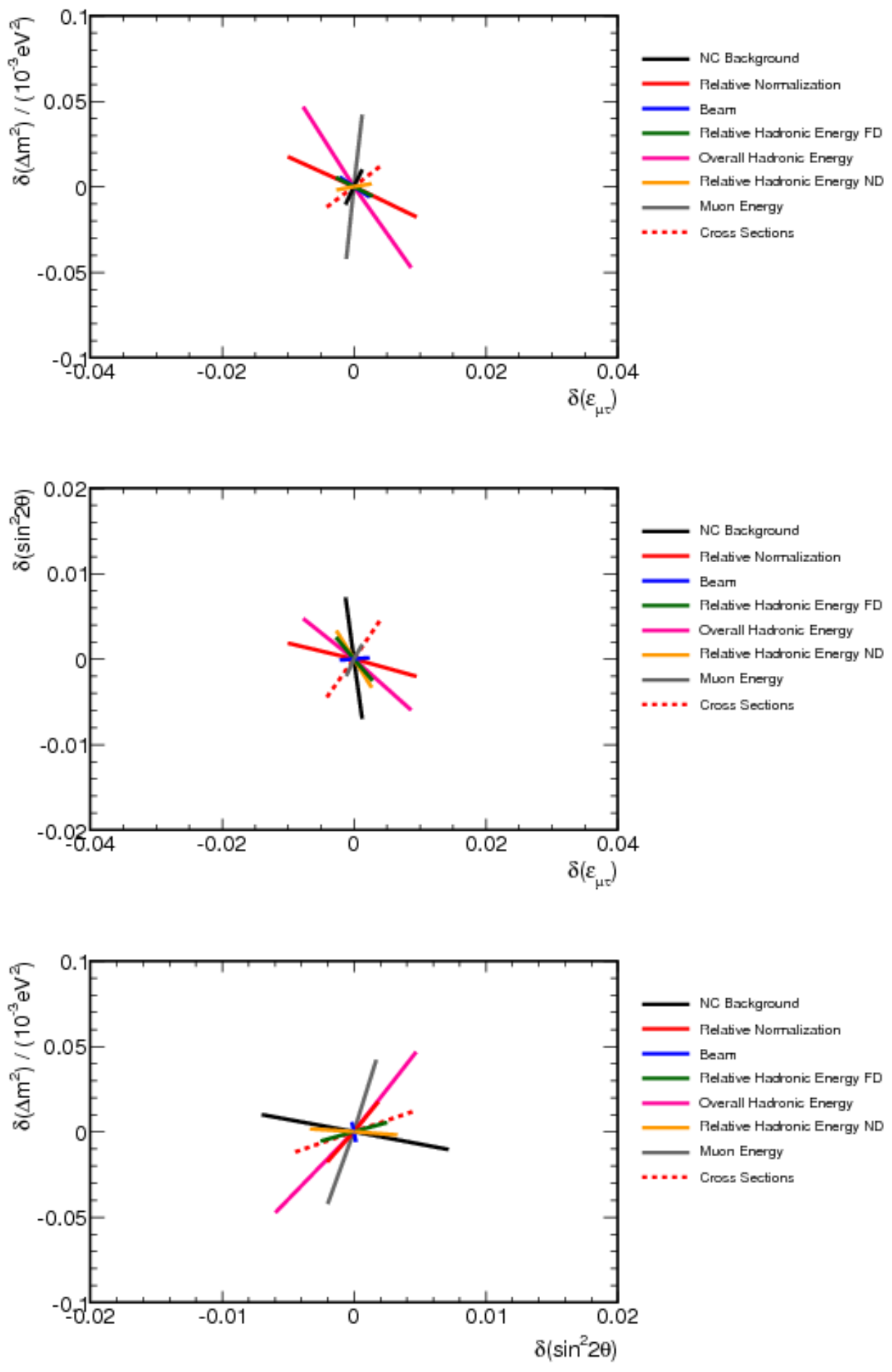

Figure 5.16: Sizes of systematic uncertainties and their effect on each parameter from a three parameter fit to oscillations with non-standard interactions. 
Although this analysis is less limited by statistics than the antineutrino-only oscillations analysis, systematic uncertainties are still small compared to the statistical uncertainty, Fig. 5.17 compares systematic uncertainties to the statistical contour in $\Delta m^{2}$ vs. $\epsilon$ space. The statistical uncertainty completely covers the systematics.

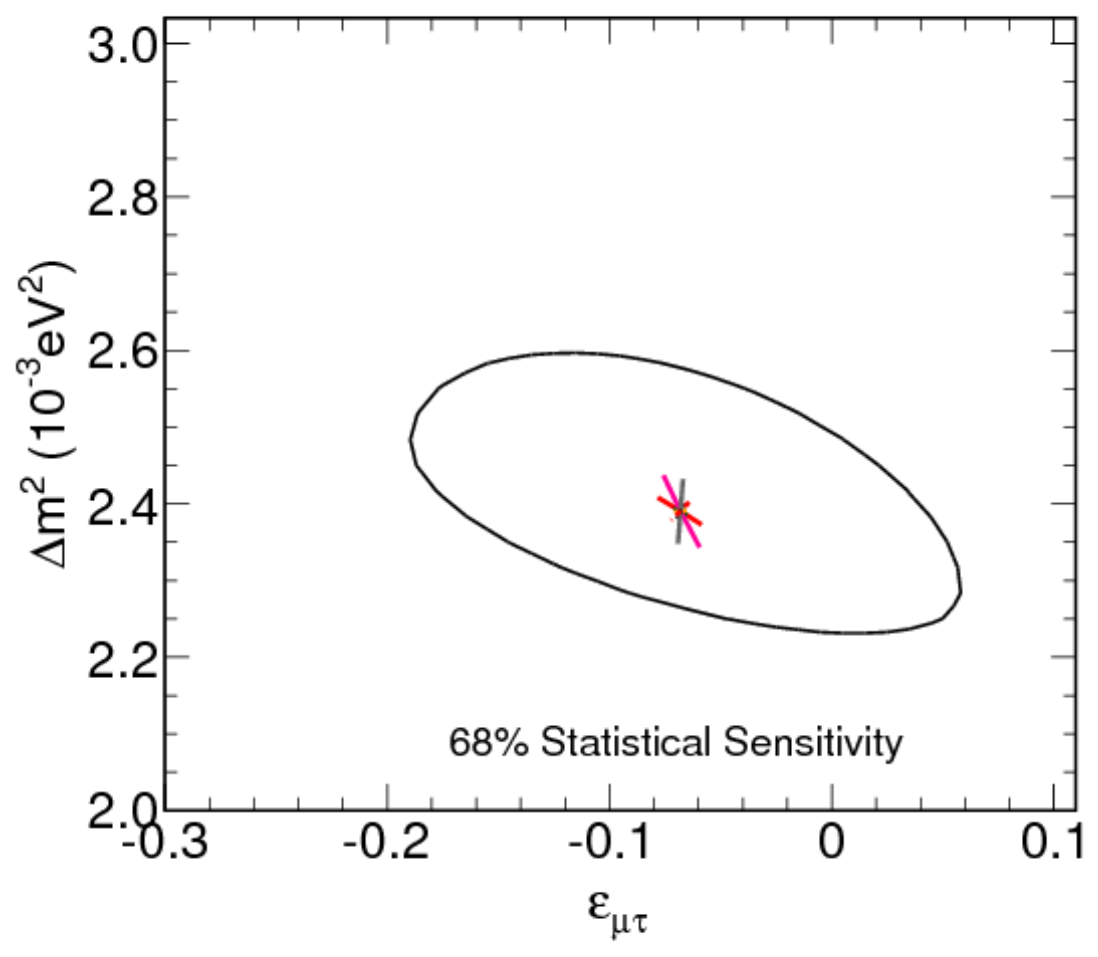

Figure 5.17: Size of systematic uncertainties in the NSI analysis compared to the $68 \%$ statistical uncertainty. 


\subsection{CONCLUSION AND OUTLOOK}

This thesis presents the most precise long-baseline measurement of muon antineutrino disappearance to date with a tagged $\bar{\nu}_{\mu}$ beam. The antineutrino dataset collected in two run periods for a total exposure of $2.95 \times 10^{20}$ POT has been analyzed and found to support the hypothesis of oscillations. With a no-oscillations expectation of 273 events and an observation of 193, antineutrino oscillation parameters are measured to be $\left|\Delta \bar{m}_{\text {atm }}^{2}\right|=2.62_{-0.28}^{+0.31} \times 10^{-3} \mathrm{eV}^{2}$ and $\sin ^{2}\left(2 \bar{\theta}_{23}\right)=0.95_{-0.11}^{+0.10}$ with $\sin ^{2}\left(2 \bar{\theta}_{23}\right)>0.75$ at $90 \%$ C.L. This is the most precise measurement to date of the atmospheric mass splitting $\Delta \bar{m}_{\mathrm{atm}}^{2}$. The null oscillation hypothesis

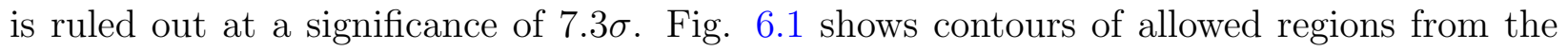
current and previous analyses as well as those of neutrino oscillations.

CPT symmetry requires that masses of particles and their antiparticles be identical. The mass splitting is therefore expected to be identical for neutrinos in a CPT-conserving scenario without any non-standard effects. A previous MINOS analysis using only $1.71 \times 10^{20}$ POT of antineutrino data found parameters of $\left|\Delta \bar{m}_{\text {atm }}^{2}\right|=3.36_{-0.40}^{+0.46} \times 10^{-3} \mathrm{eV}^{2}$ and $\sin ^{2}\left(2 \bar{\theta}_{23}\right)=$ $0.86_{-0.12}^{+0.11}$, with which the likelihood that neutrinos and antineutrinos have identical underlying oscillation parameters is $2 \%$. The results of this analysis are in much better agreement with neutrino oscillations with a $42 \%$ statistical likelihood of identical oscillation parameters. The two antineutrino analysis techniques are very similar; it can be concluded that the previous measurement of a high $\Delta m_{\mathrm{atm}}^{2}$ is most likely a statistical fluctuation. Contours from the two separate antineutrino run periods shown in Fig. 6.2 have substantial overlap and lie on either side of the neutrino oscillation allowed region, converging on a value in agreement with neutrinos when combined to increase the dataset. This also suggests that the deviation from the CPT-conserving expectation with small datasets is a statistical effect.

This thesis also uses the $7.1 \times 10^{20}$ POT neutrino dataset in combination with the antineu- 


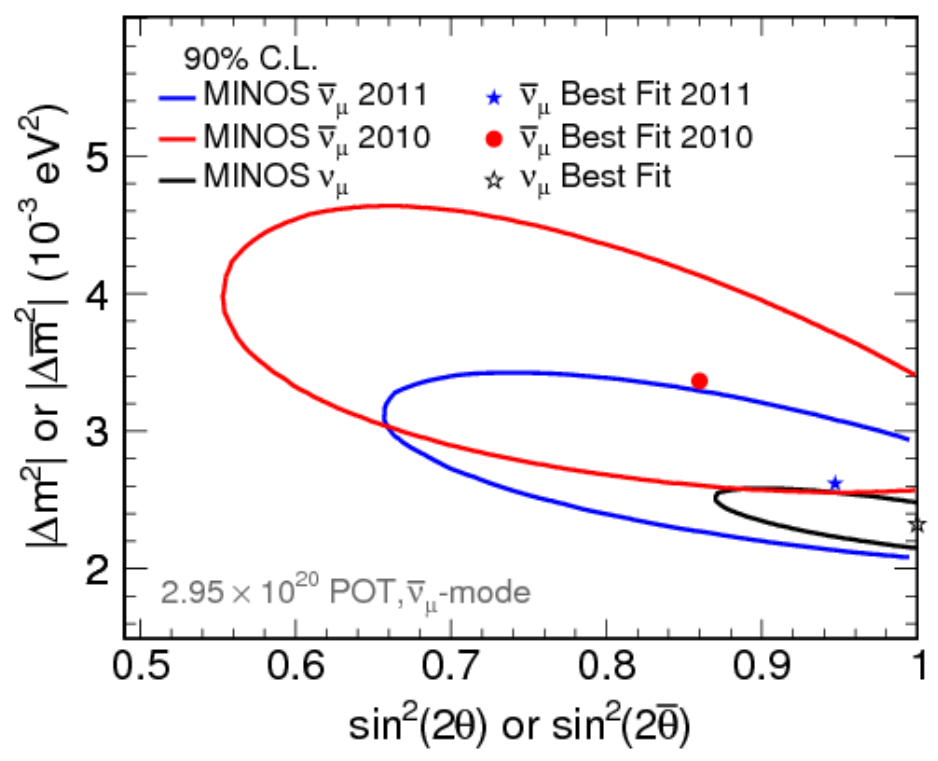

Figure 6.1: 90\% C.L. contours for the previous antineutrino oscillation result published by MINOS [111] in red and the current measurement in blue, overlaid with the $90 \%$ neutrino contour [105].

trino data to constrain non-standard interactions which would alter the survival probability as described by Eq. (1.85). No evidence for such non-standard interactions is found. The best fit to this model gives $\epsilon_{\mu \tau}=-0.068_{-0.080}^{+0.083}$ with a common mass splitting and mixing angle of $\left|\Delta m_{\text {atm }}^{2}\right|=2.39_{-0.105}^{+0.135} \times 10^{-3} \mathrm{eV}^{2}$ and $\sin _{23}^{2}(2 \theta)>0.91$ (90\% C.L.). This measurement also supports the conclusion that neutrino and antineutrino oscillation parameters agree within the statistical uncertainty. This result is also in agreement with other measurements of non-standard interactions [65] as well as values of $\epsilon$ extracted from global fits to data from multiple experiments [38, 37, 112, 33, 63].

The significance of the non-standard interactions measurement presented here is twofold. First, it is a direct measurement from a single experiment where the effect of systematics is well understood and taken into account. Second, neutrino and antineutrino chargedcurrent interactions can be identified event-by-event in MINOS giving this analysis a unique 


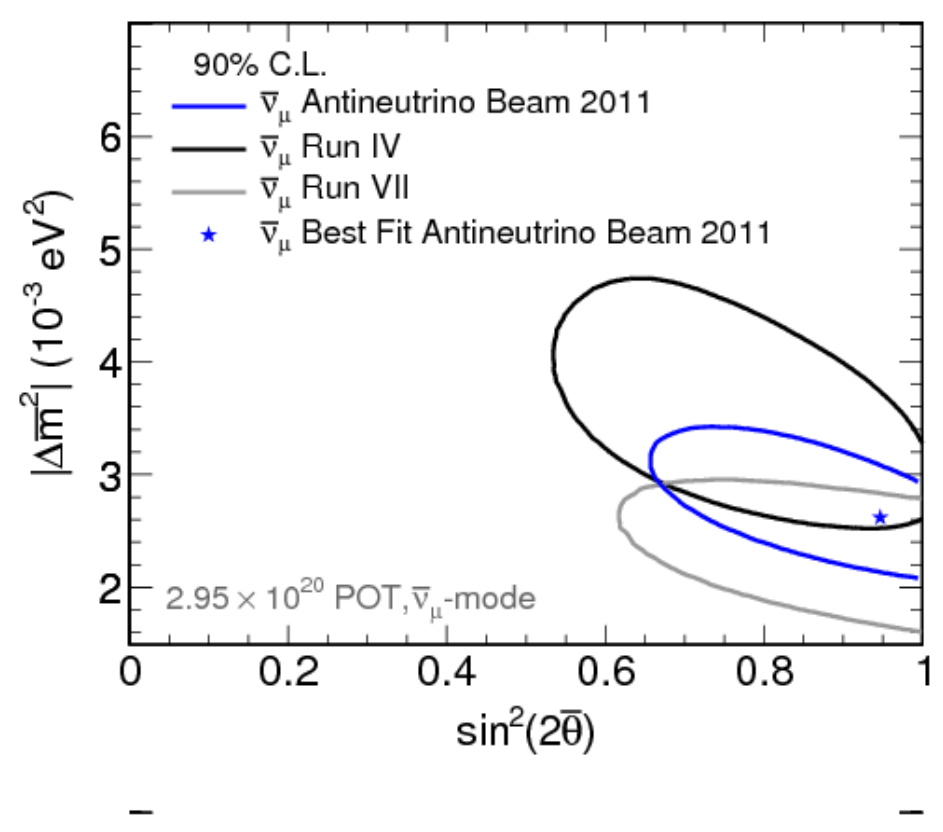

Figure 6.2: The evolution of the $90 \%$ contours of antineutrino oscillation parameters with increasing data. Shown in black is the latest published result [111] while the gray line shows the results from the second run only. The blue contour shows the final result presented here.

opportunity to search for an effect that manifests itself as a difference between neutrinos and antineutrinos.

\subsection{FUTURE MEASUREMENTS AND EXPERIMENTS}

An upgraded version of MINOS, called MINOS+, will collect data starting in 2013 from an upgraded NuMI beam which will deliver a higher intensity of higher energy neutrinos (and antineutrinos) to the $\mathrm{NO} \nu \mathrm{A}$ experiment. With the addition of data that spans a wider range of energies, the experiment has more sensitivity to flavor conserving effects which manifest as a change in the shape of the energy spectrum rather than a change of the rate of disappearance. New results on non-standard interactions are expected with this dataset. 
Neutrino physics in general is ripe for discovery at the time of this writing. Since its postulation early in this century, studying this elusive particle has improved our understanding of many of its properties while many remain unknown. We do not know the order of the masses of neutrinos (mass hierarchy), whether or not neutrinos violate CP-symmetry, or whether they are Dirac or Majorana particles. There are limits of neutrino mass from neutrino experiments and cosmology but no precise measurements. Developing a thorough comprehension of neutrino mixing and mass is essential to building a unified theory of particle physics that bridges the quark and lepton sectors.

The task of answering the many open questions of neutrinos requires multiple ventures: solar, reactor and accelerator neutrino oscillation experiments, direct mass measurements and neutrinoless double beta decay. One of the most fundamental of these open questions is

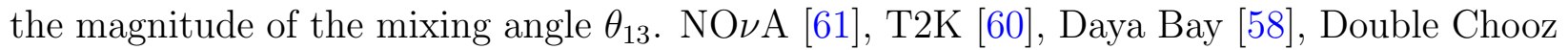
[57] and RENO [59] are some of the experiments that will shed light on this in the next few years. Knowledge of $\theta_{13}$ allows for an experiment like $\mathrm{NO} \nu \mathrm{A}$ to measure the $\mathrm{CP}$-violating phase $\delta_{C P}$ and determine the mass hierarchy. Neutrinoless double beta decay experiments are ongoing; the observation of such a decay would indicate that the neutrino is a Majorana particle (i.e. its own antiparticle). These and other future experiments will keep advancing our understanding of neutrinos, improve the Standard Model and further knowledge of the universe. 


\section{BIBLIOGRAPHY}

[1] O. Hahn and L. Meitner, "Uber die absorption der $\beta$-strahlen einiger radioelemente," Physikalische Zeitschrift, vol. 9, pp. 312-333, 1908.

[2] O. Hahn and L. Meitner, "Uber die $\beta$-strahlen des aktiniums," Physikalische Zeitschrift, vol. 9, pp. 987-704, 1908.

[3] O. Hahn, L. Meitner, and O. von Baeyer, "Uber die $\beta$-strahlen des aktivien niederschlags des thoriums," Physikalische Zeitschrift, vol. 12, pp. 273-279, 1911.

[4] O. Hahn, L. Meitner, and O. von Baeyer, "Nachweis von $\beta$-strahlen be radium d," Physikalische Zeitschrift, vol. 12, pp. 378-379, 1911.

[5] J. Chadwick, "Intensitatsverteilung im magnetischen spektrum der $\beta$-strahlen von radium b + c," Verhandlungen der deutschen physikalischen Gesellschaft, vol. 16, pp. 383-391, 1914.

[6] C. D. Ellis and W. A. Wooster, "The average energy of disintegration of radium e," in Proceedings of the Royal Society (London), vol. A117, pp. 109-123, 1927.

[7] F. Rasetti, "On the raman effect in diatomic gases," Proceedings of the National Academy of Sciences of the United States of America, vol. 15, pp. 234-237, March 1929.

[8] F. Rasetti, "On the raman effect in diatomic gases. ii," Proceedings of the National Academy of Sciences of the United States of America, vol. 15, pp. 515-519, June 1929.

[9] W. Pauli. Letter by Wolfgang Pauli to the attendees of the Tubingen conference. From the CERN archive, http://cdsweb. cern.ch/record/83282.

[10] J. Chadwick, "POSSIBLE EXISTENCE OF A NEUTRON," Nature, vol. 129, p. 312, 1932.

[11] F. L. Wilson, "Fermi's theory of beta decay," Am. J. Phys, vol. 36, no. 12, 1968.

[12] F. Reines and C. Cowan, "Detection of the free neutrino," Phys.Rev., vol. 92, pp. 830831, 1953. 
[13] C. Cowan, F. Reines, F. Harrison, H. Kruse, and A. McGuire, "Detection of the free neutrino: A Confirmation," Science, vol. 124, pp. 103-104, 1956.

[14] M. Goldhaber, L. Grodzins, and A. Sunyar, "HELICITY OF NEUTRINOS," Phys.Rev., vol. 109, pp. 1015-1017, 1958.

[15] G. Danby et al., "Observation of High-Energy Neutrino Reactions and the Existence of Two Kinds of Neutrinos," Phys. Rev. Lett., vol. 9, pp. 36-44, 1962.

[16] T. Patzak, "First direct observation of the tau-neutrino," Europhys.News, vol. 32, pp. 56-57, 2001.

[17] "Precision electroweak measurements on the $Z$ resonance," Phys.Rept., vol. 427, pp. 257-454, 2006.

[18] K. Nakamura, "Review of particle physics," J. Phys., vol. G37, p. 075021, 2010.

[19] M. E. Peskin and D. V. Schroeder, An Introduction To Quantum Field Theory (Frontiers in Physics). Westview Press, 1995.

[20] F. Halzen and A. D. Martin, Quarks and Leptons: An Introductory Course in Modern Particle Physics. Wiley, 1984.

[21] B. Pontecorvo, "Inverse beta processes and nonconservation of lepton charge," Sov.Phys.JETP, vol. 7, pp. 172-173, 1958.

[22] Z. Maki, M. Nakagawa, and S. Sakata, "Remarks on the unified model of elementary particles," Prog.Theor.Phys., vol. 28, pp. 870-880, 1962.

[23] R. Brown, U. Camerini, P. Fowler, H. Muirhead, C. Powell, et al., "Observations with electron sensitive plates exposed to cosmic radiation. 2," 1987.

[24] J. Evans, Measuring Antineutrino Oscillations with the MINOS Experiment. PhD thesis, University of Oxford, 2008.

[25] V. Kostelecky and M. Mewes, "Lorentz and CPT violation in the neutrino sector," Phys.Rev., vol. D70, p. 031902, 2004.

[26] G. Barenboim, L. Borissov, J. D. Lykken, and A. Smirnov, "Neutrinos as the messengers of CPT violation," JHEP, vol. 0210, p. 001, 2002.

[27] G. Barenboim, J. F. Beacom, L. Borissov, and B. Kayser, "CPT violation and the nature of neutrinos," Phys.Lett., vol. B537, pp. 227-232, 2002.

[28] L. Wolfenstein, "Neutrino oscillations in matter," Phys. Rev. D, vol. 17, pp. 2369-2374, May 1978.

[29] S. Mikheev and A. Smirnov, "Resonance Amplification of Oscillations in Matter and Spectroscopy of Solar Neutrinos," Sov.J.Nucl.Phys., vol. 42, pp. 913-917, 1985. 
[30] G. Gelmini and E. Roulet, "Neutrino masses," Rept. Prog. Phys., vol. 58, pp. 12071266, 1995.

[31] H. Nunokawa, S. J. Parke, and J. W. Valle, "CP Violation and Neutrino Oscillations," Prog.Part.Nucl.Phys., vol. 60, pp. 338-402, 2008.

[32] S. Davidson and V. Sanz, "Non-Standard Neutrino Interactions at Colliders," 2011.

[33] C. Biggio, M. Blennow, and E. Fernandez-Martinez, "General bounds on non-standard neutrino interactions," JHEP, vol. 0908, p. 090, 2009.

[34] S. Davidson, C. Pena-Garay, N. Rius, and A. Santamaria, "Present and future bounds on nonstandard neutrino interactions," JHEP, vol. 0303, p. 011, 2003.

[35] G. Zeller et al., "A Precise determination of electroweak parameters in neutrino nucleon scattering," Phys.Rev.Lett., vol. 88, p. 091802, 2002.

[36] J. Kopp, P. A. N. Machado, and S. J. Parke, "Interpretation of MINOS data in terms of non-standard neutrino interactions," 2010.

[37] M. Blennow, T. Ohlsson, and J. Skrotzki, "Effects of non-standard interactions in the MINOS experiment," Phys.Lett., vol. B660, pp. 522-528, 2008.

[38] M. Blennow, T. Ohlsson, and W. Winter, "Non-standard Hamiltonian effects on neutrino oscillations," Eur.Phys.J., vol. C49, pp. 1023-1039, 2007.

[39] Y. Grossman, "Nonstandard neutrino interactions and neutrino oscillation experiments," Phys.Lett., vol. B359, pp. 141-147, 1995.

[40] J. Valle, "RESONANT OSCILLATIONS OF MASSLESS NEUTRINOS IN MATTER," Phys.Lett., vol. B199, p. 432, 1987.

[41] M. Guzzo, A. Masiero, and S. Petcov, "On the MSW effect with massless neutrinos and no mixing in the vacuum," Phys.Lett., vol. B260, pp. 154-160, 1991.

[42] E. Roulet, "MSW effect with flavor changing neutrino interactions," Phys.Rev., vol. D44, pp. 935-938, 1991.

[43] S. Bergmann, M. Guzzo, P. de Holanda, P. Krastev, and H. Nunokawa, "Status of the solution to the solar neutrino problem based on nonstandard neutrino interactions," Phys.Rev., vol. D62, p. 073001, 2000.

[44] A. Gago, M. Guzzo, P. de Holanda, H. Nunokawa, O. Peres, et al., "Global analysis of the postSNO solar neutrino data for standard and nonstandard oscillation mechanisms," Phys.Rev., vol. D65, p. 073012, 2002.

[45] J. Davis, Raymond, D. S. Harmer, and K. C. Hoffman, "Search for neutrinos from the sun," Phys.Rev.Lett., vol. 20, pp. 1205-1209, 1968. 
[46] K. Hirata et al., "Observation of B-8 Solar Neutrinos in the Kamiokande-II Detector," Phys.Rev.Lett., vol. 63, p. 16, 1989.

[47] A. Abazov, O. Anosov, E. Faizov, V. Gavrin, A. Kalikhov, et al., "Search for neutrinos from sun using the reaction Ga-71 (electron-neutrino e-) Ge-71," Phys.Rev.Lett., vol. 67, pp. 3332-3335, 1991.

[48] P. Anselmann et al., "Solar neutrinos observed by GALLEX at Gran Sasso.," Phys.Lett., vol. B285, pp. 376-389, 1992.

[49] Y. Fukuda et al., "Solar neutrino data covering solar cycle 22," Phys.Rev.Lett., vol. 77, pp. 1683-1686, 1996.

[50] J. Hosaka et al., "Solar neutrino measurements in super-Kamiokande-I," Phys.Rev., vol. D73, p. 112001, 2006.

[51] J. Boger et al., "The Sudbury neutrino observatory," Nucl.Instrum.Meth., vol. A449, pp. 172-207, 2000.

[52] S. Abe et al., "Precision Measurement of Neutrino Oscillation Parameters with KamLAND," Phys.Rev.Lett., vol. 100, p. 221803, 2008.

[53] K. Hirata et al., "Experimental study of the atmospheric neutrino flux," Physics Letters $B$, vol. 205, no. 2-3, pp. $416-420,1988$.

[54] R. Wendell et al., "Atmospheric neutrino oscillation analysis with sub-leading effects in Super-Kamiokande I, II, and III," Phys.Rev., vol. D81, p. 092004, 2010.

[55] P. A. N. Machado, H. Minakata, H. Nunokawa, and R. Z. Funchal, "Combining Accelerator and Reactor Measurements of theta $a_{13}$ : The First Result," 2011.

[56] Y. Nir, "Introduction to leptogenesis," 2007.

[57] F. Ardellier et al., "Double Chooz: A Search for the neutrino mixing angle theta(13)," 2006.

[58] X. Guo et al., "A Precision measurement of the neutrino mixing angle theta(13) using reactor antineutrinos at Daya-Bay," 2007.

[59] J. Ahn et al., "RENO: An Experiment for Neutrino Oscillation Parameter $\theta_{13}$ Using Reactor Neutrinos at Yonggwang," 2010.

[60] K. Abe and J. Spitz, "The T2K Experiment," 2011. Long author list - awaiting processing.

[61] G. S. Davies and f. t. N. Collaboration, "NOvA: Present and Future," 2011. * Temporary entry *. 
[62] M. Gonzalez-Garcia, M. Guzzo, P. Krastev, H. Nunokawa, O. Peres, et al., "Atmospheric neutrino observations and flavor changing interactions," Phys.Rev.Lett., vol. 82, pp. 3202-3205, 1999.

[63] F. Escrihuela, M. Tortola, J. Valle, and O. Miranda, "Global constraints on muonneutrino non-standard interactions," Phys.Rev., vol. D83, p. 093002, 2011.

[64] K. Abe et al., "Search for Differences in Oscillation Parameters for Atmospheric Neutrinos and Antineutrinos at Super-Kamiokande," 2011. Long author list - awaiting processing.

[65] G. Mitsuka et al., "Study of Non-Standard Neutrino Interactions with Atmospheric Neutrino Data in Super-Kamiokande I and II," 2011. Long author list - awaiting processing.

[66] "Numi techical design handbook.".

[67] P. A. Rodrigues, A Sterile Neutrino Search with the MINOS Experiment. PhD thesis, University of Oxford, 2010.

[68] A. I. Himmel, Antineutrino Oscillations in the Atomospheric Sector. PhD thesis, California Institute of Technology, 2011.

[69] D. Bhattacharya, Neutrino and antineutrino inclusive charged-current cross section measurement with the MINOS Near Detector. PhD thesis, University of Pittsburgh, 2009 .

[70] L. Loiacono, Measurement of the Muon Neutrino Inclusive Charged Current Cross Section on Iron Using the MINOS Detector. PhD thesis, University of Texas at Austin, 2010.

[71] D. Michael et al., "The Magnetized steel and scintillator calorimeters of the MINOS experiment," Nucl.Instrum.Meth., vol. A596, pp. 190-228, 2008.

[72] G. Drake, J. Dawson, and C. Nelson, "Overview of the minos front end electronics for the near detector." 1999.

[73] R. Brun, F. Carminati, and S. Giani, "GEANT Detector Description and Simulation Tool," 1994. CERN Program Library Long Writeup.

[74] P. Adamson et al., "The minos calibration detector," Nuclear Instruments and Methods in Physics Research Section A: Accelerators, Spectrometers, Detectors and Associated Equipment, vol. 556, no. 1, pp. 119 - 133, 2006.

[75] P. Adamson et al., "A Study of Muon Neutrino Disappearance Using the Fermilab Main Injector Neutrino Beam," Phys.Rev., vol. D77, p. 072002, 2008. 
[76] P. Ballester, "Hough transform for robust regression and automated detection," Astron.Astrophys., vol. 286, p. 1011, 1994.

[77] J. Marshall, A Study of Muon Neutrio Disappearance with the MINOS Detectors and the NuMI Neutrino Beam. PhD thesis, University of Cambridge, 2008.

[78] D. E. Groom, N. V. Mokhov, and S. I. Striganov, "Muon stopping power and range tables 10-MeV to 100-TeV," Atom.Data Nucl.Data Tabl., vol. 78, pp. 183-356, 2001.

[79] C. Backhouse, Measuring neutrino oscillation parameters using $\nu_{\mu}$ disappearance in MINOS. PhD thesis, University of Oxford, 2011.

[80] C. Smith, "Ss event builder algorithm." Minos Doc-2821.

[81] P. Adamson, J. Alner, B. Anderson, T. Chase, P. Dervan, et al., "The MINOS light injection calibration system," Nucl.Instrum.Meth., vol. A492, pp. 325-343, 2002.

[82] P. Adamson et al., "On the linearity of the minos light-injection calibration system," Nuclear Instruments and Methods in Physics Research Section A: Accelerators, Spectrometers, Detectors and Associated Equipment, vol. 521, no. 2-3, pp. 361 -366, 2004.

[83] C. Zeitnitz and T. Gabriel, "The GEANT - CALOR interface and benchmark calculations of ZEUS test calorimeters," Nucl.Instrum.Meth., vol. A349, pp. 106-111, 1994.

[84] A. Fasso, A. Ferrari, S. Roesler, P. Sala, F. Ballarini, et al., "The Physics models of FLUKA: Status and recent developments," p. MOMT005, 2003.

[85] A. Ferrari, P. Sala, A. Fasso, and J. Ranft, "FLUKA: A multi-particle transport code (Program version 2005)," 2005.

[86] S. Agostinelli et al., "GEANT4: A Simulation toolkit," Nucl.Instrum.Meth., vol. A506, pp. 250-303, 2003.

[87] J. Allison, K. Amako, J. Apostolakis, H. Araujo, P. Dubois, et al., "Geant4 developments and applications," IEEE Trans.Nucl.Sci., vol. 53, p. 270, 2006.

[88] H. Gallagher, "The NEUGEN neutrino event generator," Nucl.Phys.Proc.Suppl., vol. 112, pp. 188-194, 2002.

[89] J. Coelho and G. Pawloski, "Incorporating intensity profile in monte carlo." Minos Doc-6032.

[90] R. Hatcher, "Active detector (fls) simulation in gminos and recominos." MINOS Doc-479v1.

[91] R. Ospanov, A measurement of muon neutrino disappearance with the MINOS detectors and NuMI beam. PhD thesis, The University of Texas at Austin, 2008. 
[92] Z. Isvan and C. Backhouse, "Selector optimization for the 2010 numubar analysis." MINOS Doc-6984.

[93] C. Alt et al., "Inclusive production of charged pions in $\mathrm{p}+\mathrm{C}$ collisions at $158-\mathrm{GeV} / \mathrm{c}$ beam momentum," Eur.Phys.J., vol. C49, pp. 897-917, 2007.

[94] M. Bonesini, A. Marchionni, F. Pietropaolo, and T. Tabarelli de Fatis, "On Particle production for high-energy neutrino beams," Eur.Phys.J., vol. C20, pp. 13-27, 2001.

[95] J. Nelson, "Magnet findings and recommendations." MINOS Doc-3678.

[96] R. Hatcher et al., "Range/Energy Task Force Position Paper." MINOS Doc-3134.

[97] J. Evans, "Absolute (calorimetric) shower energy uncertainty." Minos Doc-7173.

[98] P. Rodrigues, "Notes on the Normalization Systematic for NC and CC Analyses." MINOS Doc-6636v3.

[99] C. Andreapoulos, D. Bhattacharya, et al., "Updated Cross Section Uncertainties for the Charged Current Analysis." MINOS Doc-2989.

[100] M. Dorman, "Beam Fit Position Paper." MINOS Doc-7146.

[101] N. Gehrels, "Confidence limits for small numbers of events in astrophysical data," Astrophys.J., vol. 303, pp. 336-346, 1986.

[102] G. J. Feldman and R. D. Cousins, "A Unified approach to the classical statistical analysis of small signals," Phys.Rev., vol. D57, pp. 3873-3889, 1998.

[103] N. Devenish and A. I. Himmel, "Feldman-Cousins for the Antineutrino Analysis." MINOS Doc-5209v7.

[104] A. Radovic, "Systematics for 2011 rhc analysis." Minos Doc-8272.

[105] P. Adamson et al., "Measurement of the neutrino mass splitting and flavor mixing by MINOS," Phys. Rev. Lett., vol. 106, p. 181801, 2011.

[106] S. J. Coleman, A Measurement of Neutrino Oscillations with Muon Neutrinos in the MINOS Experiment. PhD thesis, College of William and Mary, 2011.

[107] J. Ratchford. PhD thesis, University of Texas at Austin, 2012.

[108] M. L. Strait, A Measurement of Oscillation Parameters using Antifiducial Charged Current Events in MINOS. PhD thesis, University of Minnesota, 2010.

[109] J. Mitchell, Measuring muon neutrino disappearance with the MINOS experiment. PhD thesis, University of Cambridge, 2011.

[110] J. Ratchford, "A Data Driven Estimate to the NC Background." MINOS Doc-7115v6. 
[111] P. Adamson et al., "First direct observation of muon antineutrino disappearance," 2011. accepted for publication by Phys. Rev. Lett.

[112] M. Blennow, "Non-standard interactions using the OPERA experiment," PoS, vol. NUFACT08, p. 058, 2008. 